\title{
The Analysis of Error Floor and Graphical Structure of LDPC Codes
}

by

\author{
Mehdi Karimi Dehkordi, M.Sc.
}

\author{
A dissertation submitted to the \\ Faculty of Graduate Studies and Research \\ in partial fulfillment of the requirements for the degree of
}

Doctor of Philosophy in Electrical and Computer Engineering

Ottawa-Carleton Institute for Electrical and Computer Engineering

Department of Systems and Computer Engineering

Carleton University

Ottawa, Ontario

September, 2013

(C)Copyright

Mehdi Karimi Dehkordi, 2013 
The undersigned hereby recommends to the Faculty of Graduate Studies and Research acceptance of the dissertation

\title{
The Analysis of Error Floor and Graphical Structure of LDPC Codes
}

\author{
submitted by Mehdi Karimi Dehkordi, M.Sc. \\ in partial fulfillment of the requirements for the degree of \\ Doctor of Philosophy in Electrical and Computer Engineering
}

Professor Amir H. Banihashemi, Thesis Supervisor

Professor Shu Lin, External Examiner

Professor Roshdy Hafez, Chair,

Department of Systems and Computer Engineering

Ottawa-Carleton Institute for Electrical and Computer Engineering

Department of Systems and Computer Engineering

Carleton University

September, 2013 


\section{Abstract}

To use low-density parity-check (LDPC) codes for applications with very low bit error rate requirements, it is crucial to be able to analyze and estimate the error floor performance of the codes. The error floor performance of an LDPC code is closely related to the graphical structures of the Tanner graph of the code. Two important classes of such structures are short cycles and trapping sets, which have been the main focus of many studies as well as that of this thesis.

In this thesis, we analyze the graphical structure of trapping sets and study their relationships to short cycles and to the code parameters such as block length and degree distributions. As a result, we develop new characterizations of trapping sets and novel techniques to efficiently find them.

Our study on short cycles leads to two efficient algorithms for counting them in the Tanner graph of LDPC codes, one for Tanner graphs of general LDPC codes, and one tailored for Tanner graphs of practically important quasi cyclic (QC) protograph LDPC codes. We show that for sparse graphs, the proposed algorithms significantly outperform the existing techniques, in terms of computational complexity and memory requirements. For QC protograph LDPC codes, we also derive bounds to describe the relationships between the size of the shortest cycles in the Tanner graph and some of the code parameters such as the lifting degree, and the size of the base graph. These bounds are in some cases tighter and in some other cases more general than existing bounds.

Our analysis of the graphical structure of trapping sets and their relationships to short cycles results in an efficient algorithm for finding the trapping sets of LDPC codes. The algorithm is universal in the sense that it can be applied to an arbitrary graph (regular or irregular) and that it can be tailored to find a variety of important graphical objects, such as absorbing sets. The algorithm is significantly faster than similar existing search algorithms.

We also devote special attention to elementary trapping sets (ETS), which are 
known to be the main cause of error floor for many LDPC codes. Our study leads to a simple characterization of ETSs by introducing a new property called layered superset (LSS) property. This characterization corresponds to a simple search algorithm that starts from the short cycles of the graph and finds all the ETSs with LSS property in a guaranteed fashion.

The results presented in this thesis can be used for the error floor analysis and for the design of LDPC codes with low error floors. 
To My Parents 


\section{Acknowledgments}

First af all, I would like to thank my advisor, Professor Amir Banihashemi for guiding me during my studies. Without his invaluable advice and assistance, it would not have been possible for me to complete this dissertation.

Thanks must also go to my committee members for their helpful suggestions and discussions, and for contributing their time generously to serve on my dissertation defense.

I would also like to thank my friends, especially my friends in Ottawa, who made my life richer while I was studying here.

I would especially like to thank my parents and my family for their deep love and endless support over all these years. I would not have been where I am today without their love, understanding, and efforts to support me during my research. Thank you. 


\section{Table of Contents}

Abstract $\quad$ iii

Acknowledgments $\quad$ vi

Table of Contents vii

List of Tables $\quad$ X

List of Figures $\quad$ xii

1 Introduction 1

1.1 Motivation ......................... 1

1.2 Previous Works ........................ 3

1.2.1 Counting Short Cycles . . . . . . . . . . . . 3

1.2.2 Bounds on the Girth of Protograph LDPC Codes . . . . . . . 5

1.2.3 Finding and Characterizing Dominant Trapping sets . . . . . 7

1.3 Summary of Contributions . . . . . . . . . . . . . . . . . 8

1.4 Organization of the Thesis . . . . . . . . . . . . . . . . . . . . 12

2 Background and Definitions $\quad 14$

2.1 Graphs, Walks and Cycles . . . . . . . . . . . . . . . 14

2.1.1 Bipartite Graphs . . . . . . . . . . . . . . . 15

2.1.2 Adjacency and Directed Edge Matrices . . . . . . . . . . . 16

2.2 LDPC Codes . . . . . . . . . . . . . . . . . . . 17

2.2.1 Tanner Graph . . . . . . . . . . . . . . . . . . . 17

2.2.2 Protograph LDPC Codes . . . . . . . . . . . . . . . . . . 19

2.2.3 Iterative Message Passing Algorithms . . . . . . . . . . . . . . 20

2.2.4 Importance Sampling . . . . . . . . . . . . . . . . . . . . . . . 22 
3 Message-Passing Algorithms for Counting Short Cycles in a Graph 24

3.1 Main Ideas of the Proposed Algorithm . . . . . . . . . . . . . . . . . 24

3.1.1 Extrinsic Message Passing . . . . . . . . . . . . . . . 24

3.1.2 Algorithm Development . . . . . . . . . . . . . 26

3.1.3 A Simple Example . . . . . . . . . . . . . . . . . . . 30

3.2 Proposed Message-Passing Algorithm . . . . . . . . . . . . . . 32

3.2.1 Pseudo Code . . . . . . . . . . . . . . . . . . . . . 32

3.2 .2 Parallel Implementation . . . . . . . . . . . . . . . . . . 34

3.3 Complexity of the Proposed Algorithm . . . . . . . . . . . . . . . . . 35

3.3.1 Computational Complexity . . . . . . . . . . . 35

3.3.2 Memory Requirements . . . . . . . . . . . . . . . 36

3.4 Comparison with Existing Literature . . . . . . . . . . . . . 36

3.4.1 Comparison with the Algorithm of [36] . . . . . . . . . . . 36

3.4.2 Relationship and Comparison with Matrix Multiplication Techniques ......................... 37

3.5 Numerical Results . . . . . . . . . . . . . . . . . . . . . . . . 40

4 Counting Short Cycles of Quasi Cyclic Protograph LDPC Codes 44

4.1 Proposed Method . . . . . . . . . . . . . . . . . . . 44

4.2 Numerical Results . . . . . . . . . . . . . . . . . . . . . . 47

5 On the Girth of Quasi Cyclic Protograph LDPC Codes 52

5.1 Images of Cycles of Cyclic Liftings in the Base Graph . . . . . . . . . 52

5.2 Derivation of Bounds . . . . . . . . . . . . . . . . . . 54

5.2.1 Necessary Conditions for $g \geq 6$. . . . . . . . . . . . 54

5.2.2 Necessary Conditions for $g \geq 8$. . . . . . . . . . . . . 56

5.2 .3 Necessary Conditions for $g \geq 10$. . . . . . . . . . . . 59

5.3 New Constructions of QC LDPC Codes with Girth 8 . . . . . . . . 63

5.4 More Numerical Results . . . . . . . . . . . . . . . . . . 66

6 Finding Dominant Trapping Sets of Regular LDPC Codes 68

6.1 Introduction . . . . . . . . . . . . . . . . . . . . 68

6.2 Development of the Proposed Algorithm . . . . . . . . . . . . . 68

6.2.1 Graphical Structure of Trapping Sets . . . . . . . . . . . . . . 69

6.2.2 Expansion of Elementary Trapping Sets . . . . . . . . . . . . 70 
6.2 .3 Proposed Algorithm _. . . . . . . . . . . . . . . . 73

6.2.4 Initial Set of Trapping Sets . . . . . . . . . . . . . . . 77

6.2.5 Complexity of the Algorithm . . . . . . . . . . . 78

6.2.6 Expansion of Non-Elementary Trapping Sets . . . . . . . . 80

6.3 Numerical Results . . . . . . . . . . . . . . . . . . . . . . . . . 81

7 Finding Dominant Trapping Sets of Irregular LDPC Codes $\quad 87$

7.1 Introduction . . . . . . . . . . . . . . . . . . . . . 87

7.2 On the Degree-2 Variable Nodes . . . . . . . . . . . . . . . . . 87

7.3 Finding Trapping Sets of Irregular LDPC Codes . . . . . . . . . . . 91

7.4 Numerical Results . . . . . . . . . . . . . . . . . . . . . . . . . . 94

8 On Characterization of Elementary Trapping Sets of Left-Regular $\begin{array}{ll}\text { LDPC Codes } & 99\end{array}$

8.1 Non-isomorphic Structures of ETSs . . . . . . . . . . . . . . 99

8.2 Layered Superset (LSS) Property . . . . . . . . . . . . . . . 106

8.3 Characterization of Elementary Trapping Sets of Left-Regular LDPC Codes . . . . . . . . . . . . . . . . . . . . . . . . . . 109

8.3.1 Motivating Examples . . . . . . . . . . . . . . . . . . . . 109

8.3.2 Non-Isomorphic Structures of Dominant ETSs and Their Char-

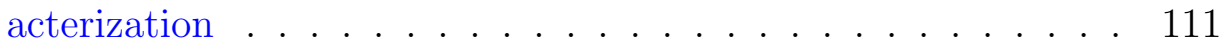

9 Conclusions and Future Works $\quad 126$

9.1 Conclusion . . . . . . . . . . . . . . . . . . . . . . . . . 126

9.2 Future Works . . . . . . . . . . . . . . . . . . . . . . . . . . 128

$\begin{array}{ll}\text { List of References } & 139\end{array}$

$\begin{array}{ll}\text { Appendix A Lemma } 20 \text { and its proof } & 140\end{array}$

$\begin{array}{ll}\text { Appendix B Lemma } 21 \text { and its proof } & 144\end{array}$

$\begin{array}{lll}\text { Appendix C Lemma } 22 \text { and its proof } & 148\end{array}$

$\begin{array}{ll}\text { Appendix D Proof of Lemma 18(i) } & 151\end{array}$

$\begin{array}{lll}\text { Appendix E Lemma } 23 \text { and its proof } & 152\end{array}$ 


\section{List of Tables}

3.1 Number of Short Cycles in the Tanner Graphs of Four Rate-1/2 LDPC Codes 41

3.2 CPU Time and Memory Requirements for the Proposed Algorithm . . . . 41

3.3 CPU Time and Memory Requirements for the Algorithm of [36] . . . . . . 42

3.4 Distribution of Short Cycles in the Tanner Graphs of Rate-1/2 Random Regular LDPC Codes with Different Degree Distributions and Different Block

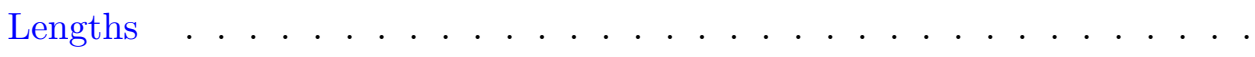

4.1 Number of Short Cycles for Different Liftings of the $3 \times 5$ Fully Connected Base Graph and the Short Cycle Distribution of a Random Graph with Similar Degree Distribution and Block Length 10,000 . . . . . . . . . .

4.2 Number of Short Cycles in the Tanner Graphs of LDPC Codes of Block Length $n=1944$ with Different Rates Adopted in IEEE 802.11 Standard [41] 49

4.3 Number of Short Cycles in the Tanner Graphs of LDPC Codes of Block Length $n=1296$ with Different Rates Adopted in IEEE 802.11 Standard [41] 49

4.4 Number of Short Cycles in the Tanner Graphs of LDPC Codes of Block Length $n=648$ with Different Rates Adopted in IEEE 802.11 Standard [41] 50

4.5 Girth Distribution of QC LDPC Codes with a $3 \times 6$ Fully Connected Base Graph and Different Lifting Degrees . . . . . . . . . . . . . . . 51

5.1 Lower Bounds on Lifting Degree for Different Girths of a Graph Lifted from a $6 \times 32$ Fully-connected Base Graph. . . . . . . . . . . . . . . . 67

5.2 Proposed Lower Bound on Lifting Degree $N_{L B}$ Compared to the Results of [99] $\left(N_{[91]}\right)$, Results of [9] and Results Found by a Heuristic Search $\left(N_{H}\right)$ for Different Girths of Graphs Lifted from Fully-connected Base Graphs with Different Sizes. . . . . . . . . . . . . . . .

6.1 Dominant Absorbing Sets(ABS) and Fully Absorbing Sets of the PEGReg252x504 Code Obtained by the Proposed Algorithm and the Exhaustive Search Algorithm of $[67]$. . . . . . . . . . . . . . 
6.2 Dominant Trapping Sets of the Tanner $(155,64)$ Code Obtained by the Proposed Algorithm . . . . . . . . . . . . . . . 83

6.3 Dominant Trapping Sets of the Margulis (2640, 1320) Code Obtained by the Proposed Algorithm and the Algorithm of [4] . . . . . . . . . . . . . 84

6.4 Dominant Trapping Sets of the $(1008,504)$ Regular LDPC Code $\left(d_{v}=\right.$ $4, d_{c}=8$ ) Obtained by the Proposed Algorithm . . . . . . . . . .

7.1 Dominant Fully Absorbing Sets of the PEGirReg252x504 Code Obtained by the Proposed Algorithm and the Algorithm of [67] . . . . . . . . . . .

7.2 Dominant Trapping Sets of the (1944, 972) Code Obtained by the Proposed Algorithm . . . . . . . . . . . . . . . . . . . 97

8.1 LSS Properties of Non-isomorphic Structures of $(a, b)$ ETS Classes for Leftregular Graphs with $d_{l}=3$ and $g=6$. . . . . . . . . . . . . . . . . 113

8.2 LSS Properties of Non-isomorphic Structures of $(a, b)$ ETS Classes for Left-regular Graphs with $d_{l}=3$ and $g=8$. . . . . . . . . . . . 117

8.3 LSS Properties of Non-isomorphic Structures of $(a, b)$ ETS Classes for Left-regular Graphs with $d_{l}=4$ and $g=6 \ldots$. . . . . . . . . 118

8.4 LSS Properties of Non-isomorphic Structures of $(a, b)$ ETS Classes for Left-regular Graphs with $d_{l}=4$ and $g=6$. . . . . . . . . . . . 119

8.5 LSS Properties of Non-isomorphic Structures of $(a, b)$ ETS Classes for Left-regular Graphs with $d_{l}=4$ and $g=8$. . . . . . . . . . . 120

8.6 LSS Properties of Non-isomorphic Structures of $(a, b)$ ETS Classes for Left-regular Graphs with $d_{l}=5$ and $g=6 . \quad$. . . . . . . . . . . 121

8.7 LSS Properties of Non-isomorphic Structures of $(a, b)$ ETS Classes for Leftregular Graphs with $d_{l}=5$ and $g=8$.

8.8 LSS Properties of Non-isomorphic Structures of $(a, b)$ ETS Classes for

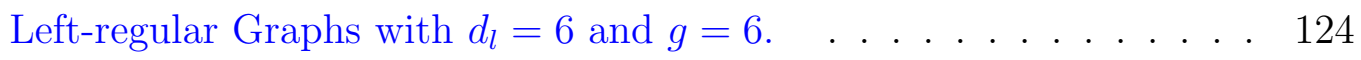




\section{List of Figures}

2.1 Two lollipop walks of length $7 \ldots \ldots \ldots$. . . . . . . . . . 15

2.2 Tanner graph of Hamming $(7,4)$ code . . . . . . . . . . . . . . 17

3.1 An extrinsic message-passing algorithm: a) messages received by $v_{1}$ at

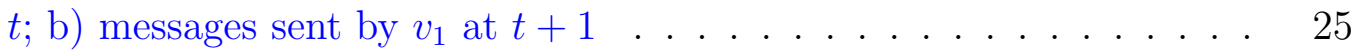

3.2 Message passing for a cycle of length $2 k$. a) initial message $X$ is passed along $e_{1}, \mathrm{~b}$ ) after $k$ iterations, $v_{1}$ receives $X$ along $e_{2} \ldots \ldots 26$

3.3 Three problematic structures for which the incoming extrinsic messages do not represent cycles. . . . . . . . . . . . . . . . . . . . . . 28

3.4 Bipartite graph of the example in Section III.C: a) $G$, b) $G$ unwound from node $u_{1}$. . . . . . . . . . . . . . . . . . . . . . . . . . . . . . . 30

3.5 Message passing of the proposed algorithm for three iterations in the graph of Figure 3.4. . . . . . . . . . . . . . . . . . . . 31

3.6 Initial message-passing in parallel implementations: a) counting short cycles of length $2 k, g / 2 \leq k \leq g-1$, b) calculating $g$ and $N_{g}$. . . . 34

5.1 Inevitable cycles: a) Theta graph, b) Dumbbell Graph. . . . . . . . . 53

5.2 Dependencies between permutation shifts of 4-cycles. . . . . . . . . 54

5.3 Dependencies between the permutation shifts of two 4-cycles having one edge in common. . . . . . . . . . . . . . . . . . . 57

5.4 Irregular base graph of Example 8. . . . . . . . . . . . . . . 59

5.5 Structures in a base graph which may be mapped to 8-cycles in the lifted graph. . . . . . . . . . . . . . . . . 60

5.6 Dependencies between permutation shifts of 4-cycles. . . . . . . . 63

6.1 The induced subgraph of a trapping set. . . . . . . . . . . . 69

6.2 Possible expansions of an elementary trapping set $\mathcal{S}$ to a larger elementary trapping set $\mathcal{S}^{\prime}$. (Unsatisfied check nodes of $G\left(\mathcal{S}^{\prime}\right)$ are not shown.) ........................... 71 
6.3 An example of a $(7,8)$ trapping set (satisfied and unsatisfied check nodes are shown by empty and full squares, respectively). . . . . . . . 74

6.4 An example explaining that the algorithm cannot find all the elementary trapping sets containing a specific elementary trapping set. . . . 75

6.5 Error floor estimation and Monte Carlo simulation for the $(1008,504)$ regular LDPC code $\left(d_{v}=4, d_{c}=8\right)$. . . . . . . . . . . . 86

7.1 Typical trapping sets constructed mostly by the degree-2 variable nodes. 89

7.2 Typical expansions of degree- 2 and degree-3 variable nodes by adding the neighboring degree-2 variable nodes. . . . . . . . . . . . . . . . . 93

7.3 Error floor estimation and Monte Carlo simulation for the $(1944,972)$ irregular LDPC code. . . . . . . . . . . . . . . . . . . . . . . . . 98

7.4 Error floor estimation and Monte Carlo simulation for the $(1000,499)$ irregular LDPC code. . . . . . . . . . . . . . . . . . . . . . 98

8.1 A $(5,4)$ elementary trapping set and its normal graph. . . . . . . . 100

8.2 All the possible non-isomorphic topologies for $(6,2)$ ETSs in leftregular LDPC codes with $d_{l}=4$ and $g=6$. . . . . . . . . . . . 101

8.3 All the possible non-isomorphic normal graphs of $(6,2)$ ETSs in leftregular LDPC codes with $d_{l}=4$ and $g=6 \ldots$. . . . . . . . . . . 101

8.4 Growing the normal graph for Case ii(a) in the proof of Proposition 5. 102

8.5 A $(6,6)$ elementary trapping set and its normal graph. . . . . . . . . 103

8.6 Possible topologies for a $(6,6)$ ETS in a left-regular graph with $d_{l}=4$ and $g=6$ : the only two possible absorbing set topologies. . . . . . . . 104

8.7 Possible topologies for a $(6,6)$ ETS in a left-regular graph with $d_{l}=4$ and $g=6$ : topologies with only one variable node connected to two unsatisfied check nodes.

8.8 Possible topologies for a $(6,6)$ ETS in a left-regular graph with $d_{l}=4$ and $g=6$ : topologies with only two variable nodes connected to two unsatisfied check nodes. . . . . . . . . . . . . . . 105

8.9 Possible topologies for a $(6,6)$ ETS in a left-regular graph with $d_{l}=$ 4 and $g=6$ : the only possible topology with three variable nodes connected to two unsatisfied check nodes. . . . . . . . . . . . . 105

8.10 (a) A $(6,6)$ ETS in a left-regular graph with $d_{l}=4$, (b) A $(7,1)$ ETS

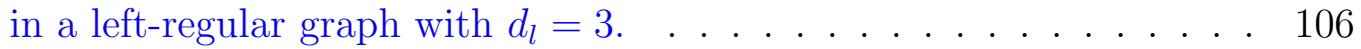

8.11 A $(5,1)$ trapping set in a left-regular graph with $d_{l}=3 \ldots$. . . . . . 109 
8.12 Two possible structures of a $(6,6)$ ETS in a left-regular graph with $d_{l}=4$ and $g=6 \ldots \ldots \ldots \ldots 111$

8.13 An $(8,2)$ ETS in a left-regular graph with $d_{l}=3$ and $g=6$ which is not an LSS of any of its cycles. . . . . . . . . . . . . . . . . . . 111

8.14 The only possible non-isomorphic structures for $(6,0)$ ETSs in leftregular graphs with $d_{l}=3$ and $g=6$, and their normal graph representations. . . . . . . . . . . . . . . . . .

8.15 Growing the normal graph of $(6,0)$ ETSs of a left-regular graph with $d_{l}=3$ and $g=6$.

8.16 A cycle of length 10 is the only possible structure for a $(5,5)$ ETS in a left-regular graph with $d_{l}=3$ and $g=6 \ldots$. . . . . . . . . . . . 116

8.17 The three structures corresponding to the "NA" cases in Table 8.1 for $(6,4),(7,3)$ and $(9,1)$ ETSs. . . . . . . . . . . . . . . 116

8.18 The only possible structure for a $(9,10)$ ETS in a left-regular graph with $d_{l}=6$ and $g=6$ that does not satisfy the LSS property. . . . .

A.1 The first four layers of the induced subgraph of a codeword of weight 6, grown from one of its variable nodes. . . . . . . . . . . . . . . . . . 141

A.2 The only possible structure for the induced subgraph of a codeword of weight 6 in a graph with variable degree 3 and girth 8. . . . . . . . . 142

B.1 The only possible structures for the induced subgraph of codewords with weight 8 in a regular Tanner graph with variable node degree 3 and girth 8. . . . . . . . . . . . . . . . . . . . 146

B.2 Graphical structure of tailless backtrackless closed walks of length 8 containing 2 copies of each of the check nodes $w_{2}$ and $w_{3}$ of the base graph. . . . . . . . . . . . . . . . . . . . 146 


\section{Chapter 1}

\section{Introduction}

\subsection{Motivation}

Low-density parity-check (LDPC) codes [33], have recently attracted much attention in the coding community. They are one of the most powerful and competitive candidates for forward error correction (FEC) in many applications. These codes have been adopted in IEEE 802.11n (WiFi) [41], IEEE 802.16e (WiMAX) [42], IEEE 802.22 (WRAN) [43], as well as 10GBASE-T Ethernet (IEEE 802.3an) [44] and digital TV broadcasting standards, DVB-T2 and DVB-S2 [24], [25]. This is mainly because LDPC codes with iterative message passing decoders can achieve excellent performance on different communication channels with relatively low computational complexity. However, there are still difficulties in using LDPC codes for applications such as optical communication and data storage which require very low bit error rates (BER), i.e., $10^{-12}$ to $10^{-20}$. The reason is a phenomenon called error floor. Error floor is a reduction in the slope of error rate curves versus signal-to-noise ratio (SNR), and may cause the code not to achieve the desired coding gain of such applications. To use LDPC codes for such applications, it is critical to have a code with a low enough error floor. Due to the lack of comprehensive knowledge on the iterative message passing decoders, it is very difficult to design practical LDPC codes with a guaranteed performance in the low error region. It is known that the performance of message passing decoding of a finite-length LDPC code in low error rate region is highly affected by certain graphical structures in the Tanner graph [102] of the code. For this reason, many studies have been focused on finding and characterizing such graphical structures. Two such structures are short cycles and trapping sets.

For a long time, it has been believed that the performance of an LDPC code is 
related to the short cycle structure of the Tanner graph. In [72], the girth distribution of the Tanner graph was related to the performance of an LDPC code. Numerous publications since have used the cycle structure of the Tanner graph as an important measure of the performance of LDPC codes, with the general belief that for good performance, short cycles should be avoided in the Tanner graph of the code. It was shown that in addition to the girth, the number and statistics of short cycles are also important performance metrics of the code [36]. In [121], error rates of finitelength LDPC codes were accurately and efficiently estimated by enumerating and testing the subsets of short cycles as error patterns. The close relationship between the performance of message passing decoders and the cycle structure of the graph, motivates the search for efficient algorithms that can enumerate cycles of different length in the graph, as well as finding their relationships to the other structures which affect the performance of such coding schemes. For structured LDPC codes, resulted from a lifting process [107], it is also important to know the relationship between the girth of an LDPC code and other code parameters such as lifting degree and the size of the base matrix. It is worth noting that all the LDPC codes currently adopted in standards are structured quasi cyclic (QC) protograph LDPC codes. In this work, we present two algorithms for counting short cycles, one for the Tanner graph of a general LDPC code, and one tailored for the Tanner graph of a QC LDPC code. We also study the effects of some of the code parameters on the girth of the resultant code.

Although short cycles are important, it should be noted that in general, not all short cycles are harmful for the performance of an LDPC code. The other graphical structures which play a more direct role in the performance of LDPC codes in the error floor region are trapping sets ${ }^{1}$. For many LDPC codes, a subset of trapping sets, called elementary trapping sets are known to be the most problematic structures [86] [16]. For a given LDPC code, the knowledge of dominant trapping sets, i.e., those that are most harmful, is very important. First, they can be used to estimate the error floor of an LDPC code. Due to the lack of analytical results for determining the error floor of LDPC codes, the performance of a given LDPC code must be verified by simulation. However, estimating the performance of LDPC codes in very low BER

\footnotetext{
${ }^{1}$ The problematic graphical structures are different for different message passing algorithms and different channels, and are called with different names such as trapping sets [86], near codewords [74], pseudo codewords [111], Zyablov-Pinsker (ZP) trapping sets [76], and absorbing sets [22]. Here, we collectively refer to such structures as trapping sets.
} 
region (i.e., $10^{-12}$ to $10^{-20}$ ) is beyond the reach of Monte Carlo simulation and is very time consuming. The solution to this problem is to use importance sampling (IS) techniques which operate by biasing the noise toward the dominant trapping sets of the code, see, e.g., [16]. Second, by knowing the dominant trapping sets, one can apply several decoder modifications to improve the error floor performance (see, e.g., [11], [35], [67]). Furthermore, the knowledge of dominant trapping sets can be used to design LDPC codes with low error floor [45], [2].

While the knowledge of dominant trapping sets is most helpful in the design and analysis of LDPC codes, attaining such knowledge, regardless of differences in the graphical structure of these sets and the sparsity of the underlying graph, is a hard problem [79], [65], [76]. The complexity of the exhaustive brute force search method for finding problematic structures of size $t$ in a code of length $n$ is proportional to $\left(\begin{array}{l}n \\ t\end{array}\right)$, which becomes quickly infeasible as $n$ and $t$ increase (e.g., for $t=10$ and $n=1000$, $\left.\left(\begin{array}{c}1000 \\ 10\end{array}\right) \approx 2.6 \times 10^{23}\right)$. It is therefore very important to look for efficient algorithms to find the trapping sets of LDPC codes. The importance of dominant trapping sets also motivates looking for methods to characterize such sets and finding their relationships to each other and to short cycles. This helps in finding more efficient algorithms to find the dominant trapping sets. In this work, we study the graphical structure of dominant trapping sets, and develop new characterizations for these sets. We also devise efficient algorithms to find them.

\subsection{Previous Works}

\subsubsection{Counting Short Cycles}

Counting and enumerating (finding) cycles in a general graph are both known to be hard problems [31]. Much work has been dedicated to lower the complexity of solving these problems. Two well-known examples of algorithms for cycle enumeration are Tarjan algorithm [103] and Johnson algorithm [46]. These algorithms have complexities $O(n|E|(\mathcal{C}+1))$ and $O((n+|E|)(\mathcal{C}+1))$, respectively, where $|E|$ is the number of edges, $n$ is number of nodes, and $\mathcal{C}$ is number of cycles in the graph. It is worth noting that the number of cycles, $\mathcal{C}$, may itself increase exponentially with $n$. Also noteworthy is that it is not straightforward to use the Tarjan and the Johnson algorithms for specifically finding cycles of a given length. Another algorithm for finding cycles is the Bax algorithm [6], which is of time complexity $O\left(2^{n}\right.$ poly $\left.(n)\right)$ and storage 
complexity $O(\operatorname{poly}(n))$. This algorithm can be easily adopted to find cycles of a given length in a graph. More recently, Schott and Staples [94], [95] studied the problems of counting and enumerating cycles of a given length $k$, also referred to as $k$-cycles, in a graph through zeon algebra. By using an adjacency matrix, called nilpotent adjacency matrix [92], [93], they demonstrated that $k$-cycles can be enumerated and counted using the $k$-th power of this adjacency matrix. Their method for enumerating $k$ cycles is of time complexity $\mathcal{O}\left(n^{\omega+k-1}\right)$ and storage complexity $\mathcal{O}\left(n^{2} 2^{n}\right)$, where $\omega \leq 3$ is the exponent representing the complexity of matrix multiplication, and is shown to be faster than the algorithms of the Tarajan and the Bax [95]. The method proposed in [94] for counting $k$-cycles in a graph with $n$ vertices has the worst case time complexity of $\mathcal{O}\left(n^{\omega+1} 2^{n}\right)$. The average time complexity of the method for counting $k$-cycles in a random simple (i.e, without parallel edges) graph is $\mathcal{O}\left(n^{\omega+1}(1+p)^{n}\right)$, where $p$ is the edge-existence probability. In the case of sparse graphs, the average complexity of the algorithm for counting $k$-cycles is $\mathcal{O}\left(n^{4}(1+q)^{n}\right)$, where $q$ satisfies $e \leq q\left(\frac{n(n-1)}{2 k}-1\right)$. It should be however noted that, these average complexities can be very large even for small values of $p$ (or $q$ ). For example, for a $(500,250)$ regular LDPC code with left-degree 3 , the average complexity of the algorithm for counting 8-cycles is $\approx 1.3 \times 10^{25}$. Still, the most prohibitive part of Schott and Staples' algorithm is its storage complexity of $\mathcal{O}\left(n^{2} 2^{n}\right)$, which increases very rapidly with $n$. In [12], Cash used the immanants of the adjacency matrix of the graph to count the $k$-cycles. Cash's method is prohibitively complex even for counting short cycles. The most computationally demanding part of his method is determining the irreducible character matrix of the symmetric group $S_{n}$. This matrix is a $P(n) \times P(n)$ matrix of integers, where $P(n) \approx \frac{\exp (\pi \sqrt{2 n / 3})}{4 n \sqrt{3}}$ (e.g., for $n=1000, P(n) \approx 2.4 \times 10^{31}$ ). Cash applied his method for counting cycles in a graph with 30 nodes, where it took three weeks to generate the matrix on a desktop computer operating at $1.4 \mathrm{GHz}$ [12].

Another approach to count or to enumerate cycles or closed walks in a graph is by using different types of zeta functions [96], [38], [97]. In [96], it is shown that closed walks of length $k$ and $k$-cycles of a graph can be counted or enumerated through finding the trace of the $k$-th power of a properly defined adjacency matrix (see also [97]). Horton [38] showed that the girth $g$ of a graph and the number of cycles with length $g$ can be determined from the Ihara zeta function of the graph.

Attempts are also made to find efficient algorithms for finding short cycles in a graph. Alon et al. [1] presented methods for counting short cycles in a general graph. 
The complexity of their algorithm however is prohibitively high for longer cycles, say beyond 7. Chang and $\mathrm{Fu}[13]$ derived an expression for the number of 6-cycles in a graph, by subtracting the number of closed walks that are not 6-cycles from the total number of closed walks of length 6 in the graph. Their expression, however, is limited to only the number of 6-cycles, and involves several summations over the elements of powers (up to 6) of the adjacency matrix. Fan and Xiao [29] presented a method for counting cycles of length $2 k, 2 \leq k \leq 5$, in the Tanner graph of LDPC codes. The complexity of their method is $O\left(\mathrm{~m}^{k+1}\right)$ where $m$ is the number of the check nodes in the graph. Their method quickly becomes prohibitively complex even for counting cycles as short as 6, particularly in graphs with large $m$. An algorithm with similar complexity was proposed in [14] for counting only the shortest cycles of a Tanner graph. Halford and Chugg [36] presented a method for counting short cycles of length $g, g+2$ and $g+4$ in bipartite graphs with girth $g$. The complexity of their method is $O\left(g n^{3}\right)$, where $n$ is the size of the larger set between the two node partitions.

\subsubsection{Bounds on the Girth of Protograph LDPC Codes}

One of the well-known classes of structured LDPC codes is protograph LDPC codes [107]. Protograph LDPC codes are constructed by lifting a smaller Tanner graph, called the base graph (see, e.g., [87]). The resultant graph after lifting is called a lifted graph. In the case of using circulant permutations in the lifting process, the resultant code is called quasi cyclic (QC). QC protograph codes are attractive for their simple implementation. A special case of protograph construction is where the base graph is a fully-connected or complete bipartite graph. In this case, each node in one partition is connected to all the nodes in the other partition of the graph. We refer to the protograph codes constructed by applying circulant permutations to the edges of such a base graph as conventional QC LDPC codes. Clearly, these codes are regular, i.e., the degrees of all the nodes in each partition of their Tanner graph are the same. Two examples of conventional QC LDPC codes are Tanner-Sridhara-Fuja (TSF) codes [100] and array-based LDPC codes [30]. It was shown in [30] that arraybased LDPC codes have very good performance in the waterfall region. These codes have also been proposed for magnetic recording [109] and digital subscriber lines [26].

Finding QC LDPC codes with large girth has been the subject of several works, for example, [59], [99], [77], [27], [115], [8] [129]. New constructions with girth 6 
as well as new methods for analyzing these codes were provided in [20] [123] [124]. In [37] and [89], some necessary conditions were presented on the number of edges in a general graph for having a girth 6 and 8, respectively. Fossorier [32] studied the cycle structure of conventional QC LDPC codes and presented some bounds on the lifting degree in order to have a certain girth. He showed that the girth of such codes is upper bounded by 12. Myung et al. [80] also studied the cycle properties of QC LDPC codes by defining block cycles and considering the overlaps between such cycles. They used this concept to obtain an upper bound on the girth of QC protograph LDPC codes, and rederived the upper bound of 12 on the girth of conventional QC LDPC codes.

Kim et al. [59] extended the concept of block cycles by defining inevitable cycles and found all the inevitable cycles of length up to 20. They also proposed a QC LDPC code construction that avoided all the inevitable cycles of length up to 12 , resulting in LDPC codes with girth larger than 12. However, the construction of [59] relies on using very large lifting degrees of $N=37^{5}$ and $N=4^{15}$, which are not suitable in practice. In [91], some necessary and sufficient conditions provided for specific girths in protograph construction using a base graph with possible parallel edges. The conditions in [91] were derived based on the smallest possible inevitable cycles in such base graphs. In the case of a base graph with no parallel edges (i.e., the case of conventional QC LDPC codes) their bounds resulted in the upper bound of 12 on the girth of the resultant graphs.

In [56] and [57], the work of [59] was extended by defining abelian-inevitable cycles, and a new proof was provided for the upper bound of 12 on the girth of conventional QC LDPC codes. In [58], the girth of conventional QC LDPC codes for the special case that the base graph is a (fully-connected) $3 \times 5$ graph and the lifting degree is a prime number of the form $15 m+1$ was studied.

In the literature listed above, except [32] and [58], only the structure of the subgraphs of the base graph that inevitably give rise to cycles in the lifted graph was studied. Such subgraphs were generally studied in isolation. In addition, in the literature, a great deal has been devoted to finding LDPC codes with girths equal to or larger than 12. Such codes generally have very sparse graphs and very large block lengths (corresponding to very large lifting degrees and/or low rates) [8], [9], [27], [59]. The girth of practical LDPC codes (of medium to high rate with short to medium block length), however, is usually less than 12 . 


\subsubsection{Finding and Characterizing Dominant Trapping sets}

The complexity of the exhaustive brute-force search method for finding problematic structures of size $t$ in a code of length $n$ becomes quickly infeasible as $n$ and $t$ increase. Efficient search algorithms have been devised to find small (dominant) stopping and trapping sets [113], [16], [88], [121], [114], [4], [67]. The reach of these algorithms however is still very limited. For example, the complexity of the algorithm of [113], [114] is only affordable for codes with lengths up to $\sim 500$. Even for these lengths, the algorithm can only find trapping sets of maximum size 11 with only one or two unsatisfied check nodes. This is while for many codes, some of the dominant trapping sets may have larger size and/or more than two unsatisfied check nodes. In [110] and [81], the authors proposed to build a database of all possible configurations for trapping sets of different sizes in a graph with specific degree distribution and girth. They then used a parent-child relationship between the trapping sets of different sizes to simplify the search of the larger trapping sets. This method was used to find the dominant trapping sets of variable-regular LDPC codes with variable degree 3 [110]. The method proposed in [110] however becomes very complex when the degree of variable nodes, and in turn the number of possible configurations increases. The application of this method becomes even more difficult when dealing with irregular LDPC codes since for such codes, there may be a large number of possible configurations for each type of trapping set, due to the variety of variable node degrees. Even for the variable-regular graphs with small variable degrees, the number of possible configurations becomes quite large for the larger trapping sets.

Many studies have also been done on the (partial) characterization of dominant trapping sets [110] [23], [71], [40], [19]. Asymptotic analysis of trapping sets has been carried out in [78], [5], [83], [64], [63], [23].

Laendner et al. [71] studied the characterization of small $(a, b)$ trapping sets of size up to $8(a \leq 8)$ and $b / a<1$ in LDPC codes from Steiner Triple Systems (STS). STS LDPC codes are a special category of regular LDPC codes with variable node degree 3. Huang et al. [40] showed that for a regular LDPC code with variable node degree $\rho$ and check node degree $\gamma$, and girth $g \geq 6$, no $(a, b)$ trapping set with $a \leq \rho$ and $b \leq \gamma$ can exist. They also studied the trapping sets of Euclidean Geometry (EG) LDPC codes and provided some bounds on the size and the number of unsatisfied check nodes of the trapping sets of such codes [19]. An EG-LDPC code with parameter $q$ is a regular LDPC code of length $q^{2}$, with variable node degree $q+1$ and check 
node degree $q$. A consequence of the bounds derived in [19] is that for the case where $q=2^{s}$, there is no trapping set of size smaller than the minimum distance of the code, i.e., $2^{s}+2$, with less than $2^{s}+1$ unsatisfied check nodes. A subset of trapping sets, called absorbing sets, for array-based LDPC codes with variable node degrees 2, 3 and 4, were studied in [23]. Absorbing sets are trapping sets in which each variable node is connected to more satisfied than unsatisfied check nodes in the induced subgraph of the set [23], [120]. Array-based LDPC codes are a subclass of (regular) protograph LDPC codes which are constructed by lifting a fully-connected base graph using cyclic permutations. The analysis in [23] was focused on minimal absorbing sets, i.e., the ones with the smallest size and with the smallest number of unsatisfied check nodes for a given size. Vasic et al. [110] studied the topological structure of trapping sets of size up to 8 in regular LDPC codes with variable node degree 3 , and proposed a hierarchical search method to find them.

\subsection{Summary of Contributions}

Our main contributions in this thesis, which are published and submitted for publication in [47], [48], [49], [50], [51], [52], [53], [54] and [55], can be summarized as follows:

- Developing an efficient message passing algorithm for counting short cycles in the Tanner graphs of LDPC codes, which can also be used for counting short cycles in a general graph.

- Devising an even more efficient method for counting short cycles in the Tanner graphs of quasi cyclic (QC) protograph LDPC codes, based on the eigenvalues of the directed edge matrix of the Tanner graphs.

- Studying the structures of base graphs which are mapped to short cycles in protograph LDPC constructions, and deriving new tighter bounds on the required lifting degree to achieve a certain girth in protograph construction using a given base matrix.

- Studying the relationship between dominant trapping sets and short cycles, lowdegree variable nodes and cycles with low approximate cycle extrinsic message degree (ACE) [105] in regular and irregular LDPC codes. 
- Developing efficient algorithms to find dominant trapping sets of regular and irregular LDPC codes.

- Identifying all the non-isomorphic structures of elementary trapping sets (ETSs) of variable-regular LDPC codes, developing a simple characterization of dominant classes of ETSs by introducing a new property called layered superset (LSS) property, and devising a simple search algorithm that starts from the short cycles of the graph and finds all the ETSs with LSS property in a guaranteed fashion.

In the first part of the thesis, we study the short cycles and present an algorithm that counts the cycles of length $g, g+2, \ldots, 2 g-2$ in a Tanner (bipartite) graph, where $g$ is the girth of the graph. The algorithm is based on message-passing on the edges of the graph, where the messages are computed at the nodes with integer additions and subtractions. The algorithm can also be applied to general (non-bipartite) graphs to count cycles of length $g, g+1, \ldots, 2 g-1$. The complexity of the proposed algorithm grows as $O\left(g|E|^{2}\right)$, where $|E|$ is the number of edges in the graph. For sparse graphs, the proposed algorithm significantly outperforms the existing algorithms, tailored for counting short cycles, in terms of computational complexity and memory requirements. As an example, for a regular graph with node degrees 3 and 6 corresponding to an $(8000,4000)$ LDPC code, the proposed algorithm is more than 30 times faster than the method of [36] and requires less memory by a factor of about 600 . We also discuss a more generic and basic approach of counting short cycles which is based on matrix multiplication, and provide a message-passing interpretation for such an approach. We then demonstrate that an efficient implementation of the matrix multiplication approach has essentially the same complexity as the proposed message-passing algorithm.

We also consider QC protograph LDPC codes as a special case, and devise an even more efficient method for counting short cycles of the Tanner graphs of such codes. The method is based on the relationship between the number of short cycles in the graph and the eigenvalues of the directed edge matrix of the graph. We demonstrate that for a QC protograph LDPC code, the complexity of computing such eigenvalues can be reduced significantly by representing the directed edge matrix as a block circulant matrix. Numerical results are presented to show the lower complexity of the proposed method compared to the existing algorithms for counting short cycles. We then use the proposed algorithm to investigate the short cycle distribution of 
a few ensembles of QC protograph LDPC codes and demonstrate that, on average, they have a superior short cycle and girth distribution compared to similar randomly constructed codes.

Moreover, we study the relationships between the girth of the Tanner graph of a quasi cyclic (QC) protograph low-density parity-check (LDPC) code, the lifting degree, the size and the structure of the base graph. For this, we closely examine the relationships among the subgraphs of the base graph that give rise to short cycles in the lifted graph. As a result, for a given base graph, we derive a lower bound on the lifting degree as a necessary condition for the lifted graph to have a certain girth. This also provides an upper bound on the girth of the family of graphs lifted from a given base graph with a given lifting degree. Unlike the other bounds which are only based on the inevitable cycles, the proposed upper bound is based on the dependency of the circular permutation shifts of short cycles in the base graph. The upper bounds derived in this thesis, which are applicable to both regular and irregular base graphs with no parallel edges, are in some cases more general and in some other cases tighter than the existing bounds. As an example, we show that for the TSF $(155,64)$ code [100], which is constructed by lifting a $3 \times 5$ fully-connected base graph with a lifting degree 31 , the largest possible girth is 8 , regardless of the permutations used. This improves the upper bound of 12 , derived based on inevitable cycles, and the upper bound of 10, derived in [101] from the well-known tree bound [33]. The results presented in this work can be used to design cyclic liftings with relatively small degree and relatively large girth. As an example, we present new QC protograph LDPC code constructions with girth 8 using fully-connected base graphs. These constructions provide upper bounds on the lifting degree required for achieving girth 8 using fully-connected base graphs.

Afterwards, we study the problematic graphical structures that dominate the error floor performance of LDPC codes, collectively referred to as trapping sets, and demonstrate that they all contain at least one short cycle (with a small exception of some of the trapping sets of irregular LDPC codes with degree-2 variable nodes). By examining the relationships between cycles and trapping sets, we devise an efficient algorithm to find dominant trapping sets of an LDPC code. The algorithm is initiated by a set of short cycles as input. Each cycle is then expanded recursively to trapping sets of increasing size in a conservative fashion, i.e., the expanded sets all have the smallest size larger than the size of the current set, and each of them will be 
used as a new input to the next step of the algorithm. It should be mentioned that although our algorithm uses the topological relationships between the small trapping sets and the larger ones, it is different from the method of [110] in several ways. Our algorithm is not based on the knowledge of the exact structure of trapping sets, and hence does not need to build a database. In fact, instead of checking all the possible configurations to find the existing ones in a graph, it directly and efficiently finds those existing configurations, and so it is much faster. Moreover, unlike the method of [110], our algorithm uses a general framework for all the degree distributions and girths (with a small exception of some of the trapping sets of irregular LDPC codes with degree-2 variable nodes). The proposed algorithm is applicable to any Tanner graph (structured or random) and can be tailored to find a variety of graphical structures, such as elementary trapping sets and absorbing sets among others. It is worth noting that the only parameters used by the proposed algorithm are the number of variable nodes and the number of unsatisfied check nodes of a trapping set. That is, the trapping sets found by the algorithm are independent of the channel model, quantization scheme, decoding algorithm and the relative positioning of the sets with respect to the rest of the graph. Results on several LDPC codes verify the high efficiency and accuracy of the algorithm. For example, for the tested codes, the search speed is improved by a factor of 10 to 100 compared to the methods of [4] and [16].

We also study the characterization of dominant trapping sets. The study of the graphical structure of trapping sets and the characterization of them so far has been mainly limited to structured codes, codes with certain variable node degrees, and to relatively small trapping sets. In this work, for the category of variable-regular LDPC codes with a certain variable node degree and a given girth, we study the topological structure of $(a, b)$ ETSs for given values of $a$ and $b$, where $a$ is the number of variable nodes and $b$ is the number of odd-degree check nodes in the induced subgraph of the ETS, and we find all the non-isomorphic structures of such ETSs. A careful examination of these structures, which are independent of the check node degree distribution of the code, reveals that for relatively small values of $a$ and $b$, the structures are all layered supersets (LSS) of small cycles, i.e., they can be characterized by a nested sequence of ETSs which starts from a short cycle and grows to the ETS one node at a time. The LSS property lends itself to a simple search algorithm that starts from short cycles of the code's Tanner graph as input and can find all the ETSs with LSS property in a guaranteed fashion. Although the general approach 
discussed in this thesis can be applied to any category of variable-regular LDPC codes with arbitrary variable node degree $d_{l}$ and girth $g$ and to any class of ETSs with arbitrary values of $a$ and $b$, the results presented here are for $d_{l}=3,4,5,6$, $g=6,8$, and $a, b \leq 10$. One of the main advantages of the results presented in this thesis is that they are applicable to specific codes, rather than just to an ensemble or a category of codes. In particular, the search algorithm based on LSS property can be used to efficiently find the dominant ETSs of a code in a guaranteed fashion. This, for example, would imply having a faster and more accurate estimation of error floor for the code under consideration using techniques such as importance sampling. Moreover, the results presented here can be used in the design of LDPC codes with low error floor. This can be achieved by avoiding certain dominant ETSs in the Tanner graph of the code. In such a context, this work can help in identifying the dominant ETSs.

It has been known that dominant trapping sets of LDPC codes have a close relationship with short cycles in the code's Tanner graph [121], [122], [52]. This work takes a rigorous step in establishing such a relationship. In general, in comparison with existing results on characterization of trapping sets such as [23], [110], the results presented here are more general in terms of being applicable to both structured and random codes, and to cover a wider range of variable node degrees and trapping set classes.

\subsection{Organization of the Thesis}

The rest of the thesis is organized as follows. Basic definitions, notations and backgrounds are provided in Chapter 2. In Chapter 3, we develop the message passing algorithm for counting short cycles in the Tanner graph of a general LDPC code. In Chapter 4, the proposed algorithm for counting short cycles in the Tanner graph of QC protograph LDPC code is presented. In Chapter 5, the relationships between the girth and the other code parameters in QC protograph construction are studied, and new bounds on the girth of resultant codes are presented. In Chapter 6, we study the graphical structures of trapping sets in regular LDPC codes, and devise an efficient algorithm to find them. Chapter 7 includes the generalization of the work of Chapter 6 to irregular LDPC codes. We then study the characterization of dominant elementary trapping sets in variable-regular LDPC codes in Chapter 8. Conclusion 
and some ideas for future work are presented in Chapter 9. 


\section{Chapter 2}

\section{Background and Definitions}

\subsection{Graphs, Walks and Cycles}

An undirected Graph $G=(V, E)$ is defined as a set of nodes $V$ and a set of (undirected) edges $E$, where $E$ is some subset of the pairs $\{\{u, v\}: u, v \in V, u \neq v\}$. In this definition and without loss of generality in the context of this thesis, we exclude loops using the condition $u \neq v$. Parallel edges are also indistinguishable by this definition and are excluded for simplicity. A directed edge $e$ is specified by an ordered pair of nodes $(u, v)$. Nodes $u$ and $v$ are called the tail and the head of $e$ and are denoted by tail(e) and head(e), respectively. The number of edges connected to a node $v$ is called the degree of $v$, and is denoted by $d_{v}$.

The graphs $G_{1}=\left(V_{1}, E_{1}\right)$ and $G_{2}=\left(V_{2}, E_{2}\right)$ are isomorphic if there is a bijection $f: V_{1} \rightarrow V_{2}$ such that nodes $v_{1}, v_{2} \in V_{1}$ are joined by an edge if and only if $f\left(v_{1}\right)$ and $f\left(v_{2}\right)$ are joined by an edge.

A walk of length $k$ in $G$ is a sequence of nodes $v_{1}, v_{2}, \ldots, v_{k+1}$ in $V$ such that $\left\{v_{i}, v_{i+1}\right\} \in E$ for all $i \in\{1, \ldots, k\}$. Equivalently, a walk of length $k$ can be described by the corresponding sequence of $k$ edges. A walk is a path if all the nodes $v_{1}, v_{2}, \ldots, v_{k}$ are distinct. A walk is called a closed walk or a cycle if the two end nodes are identical, i.e., if $v_{1}=v_{k+1}$ in the previous description. It should be noted that in this definition of a cycle, the nodes $v_{i}, 1 \leq i \leq k$, are not necessarily distinct. Consider a cycle $c$ of length $\ell(c)=k$ represented by the sequence of edges $e_{i_{1}}, e_{i_{2}}, \ldots, e_{i_{k}}$. The cycle $c$ is backtrackless if $e_{i_{s}} \neq e_{i_{s+1}}$ for any $s \in\{1, \ldots, k-1\}$. The cycle $c$ is tailless if $e_{i_{1}} \neq e_{i_{k}}$. The cycle $c$ is called primitive if $c$ is not obtained by going $r>1$ times around some other cycle $b$ (i.e., $c \neq b^{r}$ ). Two cycles of the same length are equivalent if both have the same set of edges and nodes and one is obtained by changing the 
starting node of the other one. A cycle $c$ is called simple if all the nodes $v_{i}, 1 \leq i \leq k$, are distinct. Clearly, every simple cycle is tailless and backtrackless, but the reverse is not necessarily true. In this thesis, we are mainly interested in simple cycles unless specified otherwise. We also refer to "simple cycles" as just "cycles" in the rest of the thesis. We also use the term $t b c$ walk to refer to a tailless backtrackless closed walk.

The girth $g$ of a graph is the length of a shortest cycle in the graph. In a graph $G$, cycles of length $k$, also referred to as $k$-cycles, are denoted by $\mathcal{C}_{k}$. We use $N_{k}$ for $\left|\mathcal{C}_{k}\right|$, where equivalent cycles are counted only once.

To each undirected walk (cycle), we associate two directed walks (cycles), depending on which end node or edge is selected as the starting point. This concept is important in the description of the proposed algorithm in Chapter 3 since the direction of edges is of consequence in message-passing algorithms.

In a graph $G=(V, E)$, a lollipop walk of length $k$ is defined as a sequence of nodes $v_{1}, v_{2}, \ldots, v_{k+1}$ in $V$ such that $v_{1}, v_{2}, \ldots, v_{k}$ are distinct, $v_{k+1}=v_{m}$ for some $m \in[2, k]$, and $\left(v_{i}, v_{i+1}\right) \in E$ for all $i \in\{1, \ldots, k\}$. Figure 2.1 shows two lollipop walks of length 7. The lollipop walk in Figure 2.1(a) is represented as $v_{1} v_{2} v_{3} v_{4} v_{5} v_{6} v_{7} v_{2}$. A cycle can be considered as a special lollipop walk if the definition is extended to $m=1$.

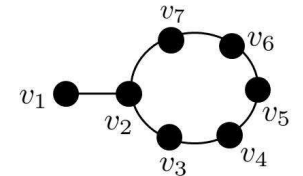

(a)

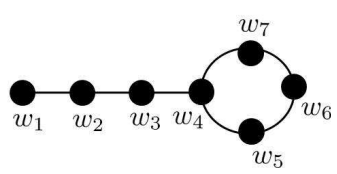

(b)

Figure 2.1: Two lollipop walks of length 7 .

\subsubsection{Bipartite Graphs}

A graph $G(V, E)$ is called bipartite if the set $V$ can be partitioned into two disjoint subsets $U$ and $W(V=U \cup W$ and $U \cap W=\emptyset)$ such that every edge in $E$ connects a node from $U$ to a node from $W$. We denote $|U|$ by $n$ and $|W|$ by $m$. Without loss of generality, we assume that $m \leq n$.

For bipartite graphs, all cycles have even lengths and girth $g$ is an even number. We call a bipartite graph $G=(U \cup W, E)$ regular if all the nodes in $U$ have the same degree $d_{u}$ and all the nodes in $W$ have the same degree $d_{w}$. Otherwise, the graph is called irregular. For a regular graph, it is easy to see $n d_{u}=m d_{w}=|E|$. 


\subsubsection{Adjacency and Directed Edge Matrices}

The adjacency matrix of a graph $G$ is the matrix $A=\left(a_{i j}\right)$, where $a_{i j}$ is the number of edges connecting node $i$ to node $j$ for all $i, j \in V$. Matrix $A$ is symmetric and since we have assumed that $G$ has no parallel edges or loops, $a_{i j} \in\{0,1\}$ for all $i, j \in V$, and $a_{i i}=0$ for all $i \in V$. One important property of the adjacency matrix is that the number of walks between any two nodes of the graph can be easily determined using the powers of this matrix. More precisely, the entry in the $i^{\text {th }}$ row and the $j^{\text {th }}$ column of $A^{k},\left(A^{k}\right)_{i j}$, is the number of walks of length $k$ between nodes $i$ and $j$. In particular, $\left(A^{k}\right)_{i i}$ is the number of closed walks of length $k$ containing node $i$, and $\operatorname{tr}\left(A^{k}\right)$ is the total number of closed walks of length $k$, where $\operatorname{tr}(\cdot)$ denotes the trace of a matrix.

For a graph $G=(V, E)$, the (Hashimato) directed edge matrix, denoted by $A_{e}$, is a $2|E| \times 2|E|$ matrix defined as follows. For every edge $e_{i} \in E$, consider two directed edges with opposite directions and denote them by $f_{i}$ and $f_{|E|+i}$. The entries $\left(A_{e}\right)_{i j}$ of $A_{e}$ are defined as [96]

$$
\left(A_{e}\right)_{i j}=\left\{\begin{array}{l}
1 \text { if } \operatorname{head}\left(f_{i}\right)=\operatorname{tail}\left(f_{j}\right) \text { and } \operatorname{head}\left(f_{j}\right) \neq \operatorname{tail}\left(f_{i}\right), \\
0 \text { otherwise. }
\end{array}\right.
$$

In particular, $\left(A_{e}\right)_{i j}=0$, for $j=i+|E|, i=1, \ldots,|E|$, and for $j=i-|E|, i=$ $|E|+1, \ldots, 2|E|$. In general, the matrix $A_{e}$ can be represented as

$$
A_{e}=\left(\begin{array}{ll}
A & B \\
C & D
\end{array}\right)
$$

where $A, B, C$ and $D$ are $|E| \times|E|$ matrices with the following properties:

a. $B=B^{T}$, and $C=C^{T}$,

b. $D=A^{T}$,

c. $b_{i i}=c_{i i}=0, \forall i \in\{1, \ldots,|E|\}$.

One important property of the directed edge matrix is that [96]

$$
\mathcal{N}_{t b c, k}=\operatorname{tr}\left(A_{e}^{k}\right) / 2 k,
$$


where $\mathcal{N}_{t b c, k}$ is the number of tailless backtrackless closed (tbc) walks of length $k$ in the graph.

\section{$2.2 \quad$ LDPC Codes}

Low Density parity check (LDPC) codes are a class of linear block code with sparse parity check matrices. An LDPC code is defined by a $m \times n$ parity check matrix $H$, where $n$ is the code length, and $m$ is the number of parity check constraints of the code. By sparse, it means that the number of non-zero elements in the parity check matrix is much smaller than the number of zero elements. The design rate of an LDPC code is $\frac{n-m}{n}$. An LDPC code can also be represented by a bipartite graph, which is called Tanner graph.

\subsubsection{Tanner Graph}

Tanner graphs are bipartite graphs to represent the parity check matrices of LDPC codes. In the Tanner representation, each column of the parity check matrix $H$ is represented by a node, called variable node, and each row of the parity check matrix is represented by to a node, called check node. There is an edge between a check node $i$ and a variable node $j$ of the Tanner graph if the entry $(i, j)$ in the parity check matrix is 1, i.e., $H(i, j)=1$. In other words, the parity check matrix $H$ is the biadjacency matrix of the Tanner graph.

Example 1. Figure 2.2 shows the parity check matrix and the Tanner graph representation of the Hamming $(7,4)$ code. In Figure 2.2, variable nodes and check nodes are represented by circles and squares, respectively.

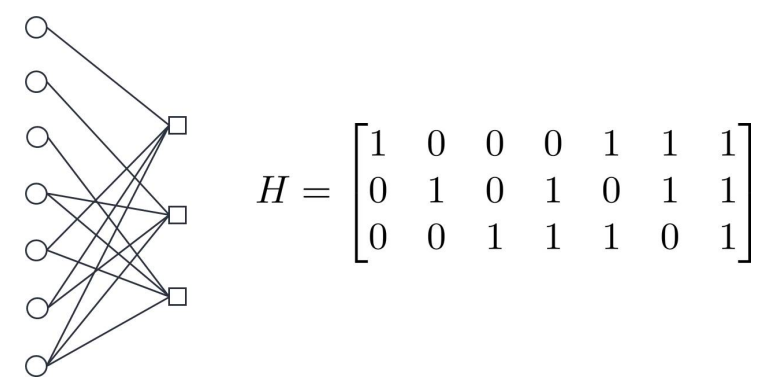

Figure 2.2: Tanner graph of Hamming $(7,4)$ code. 
For a Tanner graph $G=(U \cup W, E), U$ and $W$ are referred to as variable nodes and check nodes, respectively. A Tanner graph is also denoted by $G=(L \cup R, E)$ where $L$ is the set of variable nodes, $R$ is the set of check nodes. The notations $L$ and $R$ refer to "left" and "right," respectively, pointing to the side of the bipartite graph where variable nodes and check nodes are located, respectively. For a regular Tanner graph, we refer to $d_{l}$ and $d_{r}$ as "left-degree" and the "right-degree," respectively. A Tanner graph is called variable-regular or left-regular with variable (left) degree $d_{l}$ if $d_{v}=d_{l}, \forall v \in L$. We also use the term "girth of an LDPC code" to refer to the girth of the Tanner graph of the code.

For a subset $\mathcal{S} \subset L, \Gamma(\mathcal{S})$ denotes the set of neighbors of $\mathcal{S}$ in $R$. The induced subgraph of $\mathcal{S}$, represented by $G(\mathcal{S})$, is the graph containing nodes $\mathcal{S} \cup \Gamma(\mathcal{S})$ with edges $\{(u, v) \in E: u \in \mathcal{S}, v \in \Gamma(\mathcal{S})\}$. The set of check nodes in $\Gamma(\mathcal{S})$ with odd degree in $G(\mathcal{S})$ is denoted by $\Gamma_{\mathrm{o}}(\mathcal{S})$. Similarly, $\Gamma_{\mathrm{e}}(\mathcal{S})$ represents the set of check nodes in $\Gamma(\mathcal{S})$ with even degree in $G(\mathcal{S})$. The subgraph resulting from removing the nodes of $\Gamma_{\mathrm{o}}(\mathcal{S})$ and their edges from $G(\mathcal{S})$ is denoted by $G^{\prime}(\mathcal{S})$. In this thesis, we use the terms satisfied check nodes and unsatisfied check nodes to refer to the check nodes in $\Gamma_{\mathrm{e}}(\mathcal{S})$ and $\Gamma_{\mathrm{o}}(\mathcal{S})$, respectively. Given a Tanner graph $G=(L \cup R, E)$, the following objects play an important role in the error floor performance of the corresponding LDPC code.

\section{Definition 1.}

i) A set $\mathcal{S} \subset L$ is an $(a, b)$ "trapping set" if $|\mathcal{S}|=a$ and $\left|\Gamma_{\mathrm{o}}(\mathcal{S})\right|=b$. The integer $a$ is referred to as the size of the trapping set $\mathcal{S}$.

ii) An $(a, b)$ trapping set $\mathcal{S}$ is called "elementary" if all the check nodes in $G(\mathcal{S})$ have degree one or two.

iii) A set $\mathcal{S} \subset L$ is an $(a, b)$ "absorbing set" ${ }^{1}$ if $\mathcal{S}$ is an $(a, b)$ trapping set and if all the nodes in $\mathcal{S}$ are connected to more nodes in $\Gamma_{\mathrm{e}}(\mathcal{S})$ than to nodes in $\Gamma_{\mathrm{o}}(\mathcal{S})$.

iv) A set $\mathcal{S} \subset L$ is an $(a, b)$ "fully absorbing set" if $\mathcal{S}$ is an $(a, b)$ absorbing set and if all the nodes in $L \backslash \mathcal{S}$ have strictly more neighbors in $R \backslash \Gamma_{\mathrm{o}}(\mathcal{S})$ than in $\Gamma_{\mathrm{o}}(\mathcal{S})$.

v) A set $\mathcal{S} \subset L$ is a " $k$-out trapping" set [114] if $\Gamma_{\mathrm{o}}(\mathcal{S})$ contains exactly $k$ nodes of degree one in $G(\mathcal{S})$.

\footnotetext{
${ }^{1}$ It is worth noting that this structure was also defined in [120], although the name "absorbing set" was first used by the authors of [22].
} 
vi) Let $G=(L \cup R, E)$ be a left-regular bipartite graph with left degree l. A set $\mathcal{S} \subset L$ is a "Zyablov-Pinsker (ZP) trapping set" [76] if every node of $\mathcal{S}$ is connected to less than $l-\lfloor(l-1) / 2\rfloor$ nodes in $\Gamma_{\mathrm{o}}(\mathcal{S})$.

The ZP trapping sets are the trapping sets of the Zyablov-Pinsker bit-flipping algorithm [130] over the binary symmetric channel (BSC) [76]. Moreover, for odd values of $l$, the definitions of ZP trapping sets and absorbing sets are identical.

It is important to note that Definitions $1(i i)-1(v i)$ are all special cases of a trapping set in Definition 1(i). In the rest of the thesis, therefore, we collectively refer to them as trapping sets. Distinctions will be made as necessary. Trapping sets with smaller values of $a$ and $b$ are generally believed to be more harmful to iterative decoding. Loosely speaking, such trapping sets are called dominant. To measure how harmful a trapping set really is, one can use techniques such as importance sampling [16] to measure the contribution of the trapping set to the error floor. This contribution and the dominance of a trapping set (compared to others) also depend on the channel model and the iterative decoding algorithm, as well as the detailed structure of the Tanner graph (not just the values of $a$ and $b$ ).

\subsubsection{Protograph LDPC Codes}

Protograph LDPC codes, first introduced in [107], are constructed by lifting a smaller Tanner graph, called the base graph or the protograph. The lifted graph will then be assigned as the Tanner graph of the lifted protograph code. The lifting process consists of the base graph being copied $N$ times, and a permutation being applied to each edge cluster associated to every edge of the base graph.

In the case that permutations are cyclic, the lifted code is a quasi cyclic (QC) protograph code. The QC protograph construction can be described as follows. Consider the cyclic subgroup $C_{N}$ of the symmetric group $S_{N}$ over the set of integer numbers $Z_{N} \triangleq\{0, \ldots, N-1\}$, with the group operation defined as composition. Consider the circulant permutation $\pi_{p}$ in $C_{N}$ which corresponds to $p$ cyclic shifts to the right. We refer to $p$ as the shift of the permutation $\pi_{p}$.

Consider the following construction of a graph $\tilde{G}=(\tilde{V}, \tilde{E})$ from a graph $G=$ $(V, E)$ : We first make $N$ copies of $G$ such that for each node $v \in V$, we have $N$ copies $\tilde{v} \triangleq\left\{v^{0}, \ldots, v^{N-1}\right\}$ in $\tilde{V}$. For each edge $e=\{u, w\} \in E$, we apply a circulant permutation $\pi^{e} \in C_{N}$ to the $N$ copies of $e$ in $\tilde{E}$ such that an edge $\left\{u^{i}, v^{j}\right\}$ belongs to $\tilde{E}$ if and only if $\pi^{e}(i)=j$. The set of these edges is denoted by $\tilde{e} \triangleq\left\{e^{0}, \ldots, e^{N-1}\right\}$. 
The graph $\tilde{G}$ is called a cyclic $N$-cover or a cyclic $N$-lifting of $G$, and $G$ is referred to as the base graph corresponding to $\tilde{G}$. In the sequel, we use the terms "image" and "inverse image" to refer to the projection from the lifted graph to the base graph and the mapping from the base graph to the lifted graph, respectively. In the rest of the thesis, we also use the terminology " $m \times n$ base graph" to refer to a base graph with $m$ check nodes and $n$ variable nodes. An $m \times n$ base graph is called fully-connected or complete if it is regular and if $d_{u}=m$ and $d_{w}=n$.

In this thesis, we define the edge permutations from the variable side to the check side, i.e., the set of edges $\tilde{e}$ in $\tilde{E}$ corresponding to an edge $e=\{u, w\} \in E$, where $u \in U$ and $w \in W$, is defined by $\left\{u^{i}, w^{\pi^{e}(i)}\right\}, i \in Z_{N}$. We denote the bi-adjacency matrix of $G$ by $H$, and refer to it as the base matrix. The base matrix has $m$ rows and $n$ columns.

To the lifted graph $\tilde{G}$, we associate an LDPC code $\tilde{\mathcal{C}}$, referred to as the lifted code, such that the $m N \times n N$ parity-check matrix $\tilde{H}$ of $\tilde{\mathcal{C}}$ is equal to the bi-adjacency matrix of $\tilde{G}$. More specifically, $\tilde{H}$ consists of a total of $m n$ sub-matrices $[\tilde{H}]_{i j}, 1 \leq i \leq m$, $1 \leq j \leq n$, with each sub-matrix of dimension $N \times N$, arranged in $m$ rows and $n$ columns. The sub-matrix $[\tilde{H}]_{i j}$ in row $i$ and column $j$ is the circulant permutation matrix corresponding to the edge $\left\{u_{j}, w_{i}\right\}$ when $h_{i j} \neq 0$; otherwise, $[\tilde{H}]_{i j}$ is the all-zero matrix. Following [2], we define the $m \times n$ matrix $P=\left[p_{i j}\right]$ by $[\tilde{H}]_{i j}=I^{\left(p_{i j}\right)}, p_{i j} \in Z_{N}$, where $I^{\left(p_{i j}\right)}$ is a circulant permutation matrix whose rows are obtained by cyclically shifting the rows of the identity matrix to the left by $p_{i j}$, if $h_{i j} \neq 0$, and $p_{i j}=+\infty$, otherwise. Matrix $P$, called the matrix of edge permutation shifts, or permutation shift matrix, in brief, fully describes $\tilde{H}$ and thus the cyclically lifted code $\tilde{\mathcal{C}}$.

\subsubsection{Iterative Message Passing Algorithms}

Message passing decoders are iterative algorithms that operate on Tanner graphs of LDPC codes by computing messages at the nodes of the graph and passing them along the edges to the adjacent nodes. Messages are probabilities or other quantities derived from probabilities. Our assumption is that a binary codeword $\left(x_{1}, x_{2}, \ldots, x_{n}\right)$ is transmitted using the binary phase-shift keying (BPSK) modulation. The channel is the additive white Gaussian noise (AWGN) channel, and $\left(y_{1}, y_{2}, \ldots, y_{n}\right)$ are the symbols received from the channel. It is worth noting that throughout this thesis, we use $E_{b}$ and $N_{0}$ for the average energy per information bit and the one-sided power spectral density of the AWGN, respectively. According to our assumptions, it is more 
convenient to use log likelihood ratios (LLR) to represent the messages. Using LLRs, multiplication and division in probability domain become addition and subtraction in LLR domain. We use $\alpha_{j, i}$ to denote the LLR message sent by the check node $j$ to the variable node $i$, and $\beta_{i, j}$ to denote the LLR message sent by the variable node $i$ to the check node $j$. The two most well-known message passing decoders are the sum-product (SP) decoder and the min-sum (MS) decoder. In SP, the messages are a posteriori probability (APP) values defined as

$$
\begin{gathered}
\alpha_{j, i} \triangleq \ln \frac{\operatorname{Pr}\left(i=0 \mid \beta_{i^{\prime}, j}, i^{\prime} \in \Gamma(j) \backslash\{i\}\right)}{\operatorname{Pr}\left(i=1 \mid \beta_{i^{\prime}, j}, i^{\prime} \in \Gamma(j) \backslash\{i\}\right)}=2 \tanh ^{-1}\left(\prod_{i^{\prime} \in \Gamma(j) \backslash\{i\}} \tanh \left(\beta_{i^{\prime}, j} / 2\right)\right) \\
\beta_{i, j} \triangleq \ln \frac{\operatorname{Pr}\left(i=0 \mid L_{i}, \alpha_{j^{\prime}, i}, j^{\prime} \in \Gamma(i) \backslash\{j\}\right)}{\operatorname{Pr}\left(i=1 \mid L_{i}, \alpha_{j^{\prime}, i}, j^{\prime} \in \Gamma(i) \backslash\{j\}\right)}=L_{i}+\sum_{j^{\prime} \in \Gamma(i) \backslash\{j\}} \alpha_{j^{\prime}, i},
\end{gathered}
$$

where $L_{i}$ is the channel input LLR for bit $i$, defined as:

$$
L_{i}=\ln \frac{\operatorname{Pr}\left(x_{i}=0 \mid y_{i}\right)}{\operatorname{Pr}\left(x_{i}=1 \mid y_{i}\right)} .
$$

Min-sum (MS) is a simple approximation of SP, in which the messages from check nodes to variable nodes are defined as

$$
\alpha_{j, i} \triangleq\left(\prod_{i^{\prime} \in \Gamma(j) \backslash\{i\}} \operatorname{sign}\left(\beta_{i^{\prime}, j}\right)\right) \min _{i^{\prime} \in \Gamma(j) \backslash\{i\}}\left|\beta_{i^{\prime}, j}\right| .
$$

Since mid 1990's, a great deal of research has been devoted to understanding the behavior of message passing decoders. As a result, powerful tools such as extrinsic information transfer (EXIT) charts [10] and density evolution (DE) [84] were proposed to determine the asymptotic performance of LDPC codes on memoryless channels. It was shown that the asymptotic performance depends only on the degree distribution of the underlying Tanner graph. The assumption of these tools is that the LDPC code has infinite length and the corresponding Tanner graph is cycle-free. It should be noted that if the Tanner graph of the code is cycle-free, SP and MS are optimal in the sense of minimizing bit error rate probability and codeword error probability, respectively. However, the performances of finite-length cycle-free LDPC codes are very poor [28]. That is, the Tanner graphs of practical LDPC codes always have cycle(s). In this case, the behavior of message passing decoders is difficult to analyze 
and SP and MS are no longer optimal. One proposed method to analyze the performance of LDPC codes with cycles is computation trees, introduced by Wiberg [117]. However, the size of the computation tree grows exponentially with the degrees of the nodes in the code. This results in intractable analysis even for a small number of iterations. Nonetheless, it is known that the performance of message passing decoding of a finite-length LDPC code in low error rate region is highly affected by the graphical structures in the Tanner graph.

\subsubsection{Importance Sampling}

The probability of error in a decoder (or system) is the expected value of the error indicator function over the entire space of inputs. That is

$$
P(\text { error })=E\left[I_{e}\right]=\int_{\text {all inputs }} I_{e}(y) f(y) d_{y},
$$

where $I_{e}(y)$ is the error indicator function for the input $y$, and $f(\cdot)$ is the corresponding probability density function. Using Monte Carlo simulation, the probability of error is estimated by generating $L_{1}$ samples of $y$ (according to $f$ ), and using a sample mean estimator:

$$
\hat{P}_{M C}=\frac{1}{L_{1}} \sum I_{e}(y) .
$$

The issue with Monte carlo simulation is that to estimate low error rates, the number of samples required for having enough number of errors, and thus the number of computations increase significantly. Importance sampling (IS) is a particular version of Monte Carlo simulation which can be used for low error rate (or in general, rare event) simulations. In IS, one uses a new probability density $f^{*}$ for which errors become more likely, and then the results are weighted properly:

$$
P(\text { error })=E\left[I_{e}\right]=\int_{\text {all inputs }} I_{e}(y) \frac{f(y)}{f^{*}(y)} f^{*}(y) d_{y}=E\left[I_{e} w\right],
$$

where $w(y)=\frac{f(y)}{f^{*}(y)}$. The sampler estimation is then:

$$
\hat{P}_{I S}=\frac{1}{L_{2}} \sum I_{e}(y) w(y) .
$$


If $f^{*}(\cdot)$ is chosen properly, IS results in a significant reduction in the variance of the estimator compared to the original Monte Carlo estimator. For a given $f$, there are different choices for $f^{*}$. For a message passing LDPC decoder over AWGN channel (Gaussian density), the most common choice for $f^{*}(\cdot)$ is to shift the noise mean for the variable nodes inside the dominant trapping sets [21]. Another choice is to scale the noise variance for those variable nodes. For more information on the importance sampling technique, the reader is referred to [16] [21]. 


\section{Chapter 3}

\section{Message-Passing Algorithms for Counting Short Cycles in a Graph}

In this chapter, we present an efficient message passing algorithm that counts the cycles of length $g, g+2, \ldots, 2 g-2$ in a bipartite graph, where $g$ is the girth of the graph. The algorithm can also be applied to general (non-bipartite) graphs to count cycles of length $g, g+1, \ldots, 2 g-1$. As part of this chapter, we also present an interpretation of the matrix multiplication approach of [96] as a message-passing algorithm over a trellis diagram. Moreover, we provide an efficient implementation of the matrix-multiplication approach which has basically the same complexity as the proposed message-passing algorithm.

\subsection{Main Ideas of the Proposed Algorithm}

\subsubsection{Extrinsic Message Passing}

A message-passing algorithm operates in a graph by computing messages at the nodes and passing them along the edges to the adjacent nodes. A well-known example is the sum-product algorithm operating in a factor graph [66]. Message passing algorithms often have the property that a message sent along an edge $e$ is not a function of the message previously received along $e$. We refer to this property as extrinsic messagepassing. An example is shown in Figure 3.1, where the operation at node $v_{1}$ is multiplication. Extrinsic message-passing, for example, is known to be an important property of good iterative decoders [84]. The algorithm proposed in this chapter also has this property. 
For bipartite graphs $G(U \cup W, E)$, a natural message-passing schedule is for every node in $U$ to send messages to adjacent nodes in $W$ followed by every node in $W$ to send messages to adjacent nodes in $U$. This is referred to as parallel schedule and is used often in iterative decoding algorithms. In this case, a complete cycle of message-passing from $U$ to $W$ and then from $W$ to $U$ is called one iteration. We assign discrete time $t$ to message-passing, starting from time index zero followed by positive integer values. Corresponding to a time index $t \geq 0$, we associate an iteration number $\ell=\lfloor t / 2\rfloor+1 \geq 1$. The time indices $t=2 \ell-2$ and $t=2 \ell-1$ correspond to the first and the second halves of the iteration $\ell$. We also refer to messages passed at $t=0$ as initial messages, and use the notation $m_{u \rightarrow w}^{(\ell)}$ for a message passed from node $u$ to node $w$ at iteration $\ell$. The notations $m_{u \leftarrow}^{(\ell)}$ and $m_{u \rightarrow}^{(\ell)}$ are used for the incoming and the outgoing messages to and from node $u$ along edge $e$ at iteration $\ell$, respectively.

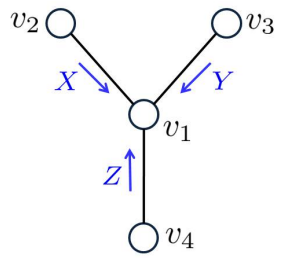

(a)

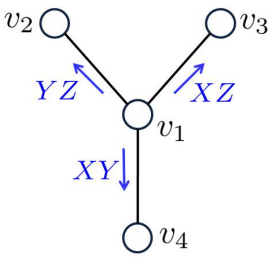

(b)

Figure 3.1: An extrinsic message-passing algorithm: a) messages received by $v_{1}$ at $t$; b) messages sent by $v_{1}$ at $t+1$

In the general context of iterative decoding, all nodes in the same partition $(U$ or $W$ ) perform the same type of operation to generate their messages. The types of operation however are usually different for the two partitions and depend on the nature of the algorithm and the domain in which the messages are presented. In the algorithm developed in this work, however, all the nodes perform the same type of operation. The messages are all monomials and the operation is multiplication. An example can be seen in Figure 3.1. In this work, a monomial is the product of integer powers of variables. For example, a message $m=X_{1}^{i} X_{2}^{j} X_{3}^{k}$ is a monomial with variables $X_{1}, X_{2}$ and $X_{3}$. We say $m$ contains $i$ copies of $X_{1}, j$ copies of $X_{2}$ and $k$ copies of $X_{3}$. If the variables are ordered, we may use a simpler representation of $m$ as a vector: $m=(i, j, k)$. Using the vector representation of messages, the multiplication of monomials is reduced to the addition of the corresponding vectors. 


\subsubsection{Algorithm Development}

Consider an extrinsic message-passing algorithm in a graph with messages as monomials and node operations as monomial multiplication. In the following, we explain how such an algorithm can count short cycles of the graph. Consider a cycle $C$ of length $2 k$ as depicted in Figure 3.2(a). Suppose that node $v_{1}$ of $C$ passes the monomial $X$ as the initial message at $t=0$ to $v_{2}$. Due to the extrinsic property of message-passing, $X$ will be passed to $v_{3}$ from $v_{2}$ at $t=1$ and continues its journey around the cycle, one node at a time, until it reaches back to $v_{1}$ at $t=2 k-1$ and at the end of iteration $k$, as shown in Figure 3.2(b). Clearly, if node $v_{1}$ had also passed a monomial $Y$ along the edge $e_{2}$ to $v_{2 k}$ at $t=0$, it would have also received $Y$ from $v_{2}$ along $e_{1}$ at the end of iteration $k$. So the iteration number at which node $v_{1}$ receives back the messages it passed at the first iteration is half the length of the cycle. The following lemma puts this basic idea in the context of the message-passing in a general graph.

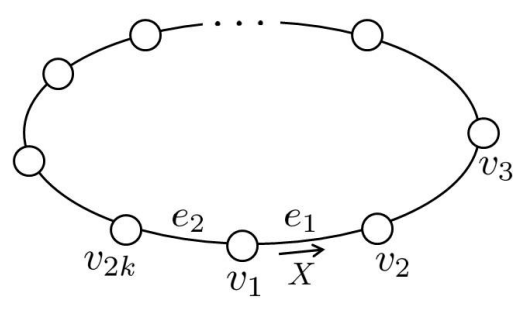

(a)

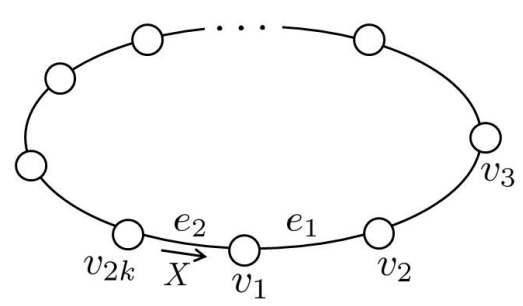

(b)

Figure 3.2: Message passing for a cycle of length $2 k$. a) initial message $X$ is passed along $\left.e_{1}, \mathrm{~b}\right)$ after $k$ iterations, $v_{1}$ receives $X$ along $e_{2}$.

Lemma 1. Suppose that $C$ is a cycle of length $2 k$ in a bipartite graph $G=(V, E)$, and $v \in V$ is in $C$. Denote the two adjacent edges of $v$ in $C$ by $e_{1}$ and $e_{2}$. Assume that the message-passing algorithm is initiated on the side of the graph which includes $v$ by passing 1 along every edge in $E$, except $e_{1}$ and $e_{2}$. For $e_{1}$ and $e_{2}$, the initial 
messages are monomials $X_{1}$ and $X_{2}$, respectively. Then, at iteration $k$, node $v$ will receive one copy of $X_{2}$ and one copy of $X_{1}$ along $e_{1}$ and $e_{2}$, respectively, where both copies have traveled through all the edges of $C$.

Proof. The proof is straightforward and follows directly from the definition of extrinsic message-passing.

It is easy to see that if the node $v$ in Lemma 1 is in $N_{2 k}^{v ; e_{1}, e_{2}}$ cycles of length $2 k$ which all include $e_{1}$ and $e_{2}$, then at iteration $k$, node $v$ will receive $N_{2 k}^{v ; e_{1}, e_{2}}$ copies of $X_{2}$ and $N_{2 k}^{v i e_{1}, e_{2}}$ copies of $X_{1}$ along $e_{1}$ and $e_{2}$, respectively, where each pair of copies has traveled through all the edges of one of the cycles, respectively. Assuming there are no additional copies of $X_{2}$ received by $v$ along $e_{1}$ and no additional copies of $X_{1}$ received by $v$ along $e_{2}$ at iteration $k$, the monomials received at iteration $k$ by $v$ along $e_{1}$ and $e_{2}$ are respectively $X_{2}^{N_{2 k}^{v i e_{1}, e_{2}}}$ and $X_{1}^{N_{2 k}^{v i e_{1}, e_{2}}}$.

We note that in addition to copies of $X_{2}$ which are received by node $v$ along $e_{1}$ at iteration $k, v$ may also receive copies of $X_{1}$ along $e_{1}$ at iteration $k$. These correspond to closed walks of length $2 k$ which start and end at edge $e_{1}$ (and are thus with tails, and clearly not cycles). To eliminate these structures in the counting process of $N_{2 k}^{v ; e_{1}, e_{2}}$, one should consider the power of received variables along $e_{1}$ and $e_{2}$ excluding the initial message. To describe this, we use the notation $m_{E, v \leftarrow}^{(k)}$ to denote the incoming message to node $v$ along $e_{1}$ at iteration $k$, excluding the variable of the initial message passed by $v$ along $e_{1}$. In the above scenario, we have $m_{E, v \leftarrow}^{(k)} e_{e_{1}}=X_{2}^{N_{2 k}^{v ; e_{1}, e_{2}}}$, and $m_{E, v \leftarrow 2}^{(k)}=X_{1}^{N_{2 k}^{v ; e_{1}, e_{2}}}$. This results in

$$
N_{2 k}^{v ; e_{1}, e_{2}}=\left\{\operatorname{ex}\left(m_{E, v \longleftarrow}^{(k)} e_{e_{1}}\right)+\operatorname{ex}\left(m_{E, v \longleftarrow}^{(k)}\right)\right\} / 2,
$$

where $\operatorname{ex}(\cdot)$ is the exponent of the monomial, defined as the sum of the powers of all its variables.

There is also a possibility that node $v$ receives additional copies of $X_{2}$ along $e_{1}$ and additional copies of $X_{1}$ along $e_{2}$ at iteration $k$. These additional copies travel either through the same cycle multiple times or through non-cycle (tailless backtrackless closed) tbc walks of length $2 k$ which start and end at $e_{1}$ and $e_{2}$, respectively. Examples of the latter structures are given in Figure 3.3, where the message $X$ is initiated at node $v_{1}$. In Figure 3.3(a), $2 k$ is in fact the sum of the lengths of the two cycles $C_{1}$ and $C_{2}$, while in Figure 3.3(b), it is the sum of the lengths of the two cycles plus twice the 


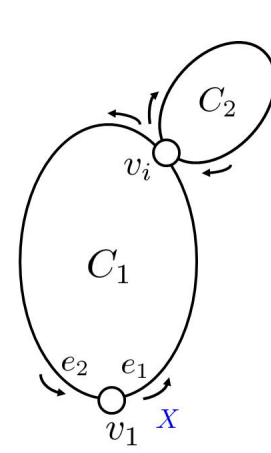

(a)

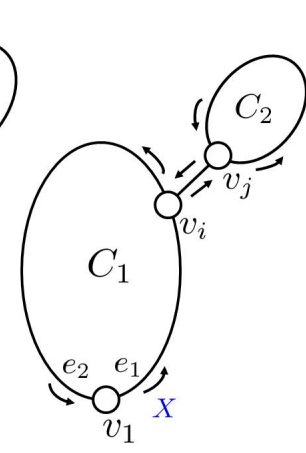

(b)

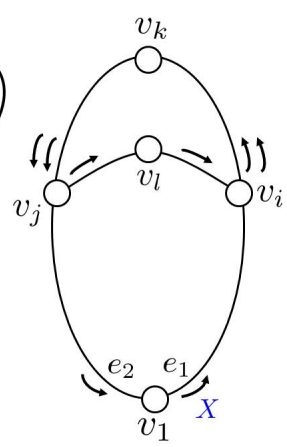

$(c)$

Figure 3.3: Three problematic structures for which the incoming extrinsic messages do not represent cycles.

length of the path between $v_{i}$ and $v_{j}$. In Figure 3.3(c), message $X$ travels from $v_{1}$ to $v_{i}$ first, and then from $v_{i}$ to $v_{j}$ through $v_{k}$. It then travels back from $v_{j}$ to $v_{i}$ through $v_{l}$ followed by a trip from $v_{i}$ to $v_{j}$ through $v_{k}$ for the second time. The journey finally ends when $X$ is passed back from $v_{j}$ to $v_{1}$. In this case, the total length of the walk is $2 k$.

A careful inspection of the problematic structures, as described above, reveals that they all include at least two cycles. This implies that the shortest length of such structures is $2 g$, where $g$ is the girth of the graph. We thus have the following results.

Lemma 2. Every tailless backtrackless closed walk of length less than $2 g$ in a graph of girth $g$ is a (simple) cycle.

Lemma 3. Consider a bipartite graph $G=(V, E)$ with girth $g$. Select a node $v \in V$ with two adjacent edges $e_{1}$ and $e_{2}$. Assume that the message-passing algorithm is initiated at $t=0$ by passing 1 along every edge in $E$, except $e_{1}$ and $e_{2}$. For $e_{1}$ and $e_{2}$ the initial messages are set to monomials $X_{1}$ and $X_{2}$, respectively. Then, at iteration $k, k<g / 2$, node $v$ will only receive 1 along all its edges including $e_{1}$ and $e_{2}$. At iteration $k, g / 2 \leq k \leq g-1$, node $v$ will receive monomials $X_{1}^{i} X_{2}^{N_{2 k}^{v ; e_{1}, e_{2}}}$ and $X_{1}^{N_{2 k}^{v ; e_{1}, e_{2}}} X_{2}^{j}$ along $e_{1}$ and $e_{2}$, respectively, where $i$ and $j$ are non-negative integers. Equation (3.1) is thus valid for $k \leq g-1$.

Proof. Node $v$ will receive messages other than 1 only if a copy of $X_{1}$ or $X_{2}$ is passed back to it. Due to the extrinsic nature of message-passing, such a copy must travel through a backtrackless closed walk with both ends at $v$. Since the length of a backtrackless closed walk is at least $g$, no messages other than 1 will be received by 
$v$ at iterations $k, k<g / 2$. At iterations $k, g / 2 \leq k \leq g-1$, node $v$ can receive copies of $X_{1}$ and $X_{2}$ that have traveled through backtrackless closed walks with both ends at $v$. In particular, the number of copies of $X_{1}$ and $X_{2}$ that $v$ receives at iteration $k \geq g / 2$, along $e_{2}$ and $e_{1}$, respectively, is equal to the number of tbc walks of length $2 k$ that start and end at $e_{1}$ and $e_{2}$. For $k$ in the range $g / 2 \leq k \leq g-1$, based on Lemma 2, such tbc walks are limited to cycles of length $2 k$ that include $e_{1}$ and $e_{2}$. (For $k \geq g$, in addition to cycles, they can include multiple trips over the same cycle or cases such as those in Fig 3.3.)

Let us now focus on the problem of counting all the cycles of a certain length $2 k$, $k<g / 2$, which pass through a certain node $v$ in a bipartite graph $G=(U \cup W, E)$. Without loss of generality, we assume $v \in U$. One approach to count all the $2 k$-cycles containing $v$ is to use Lemma 2 and count the cycles involving different adjacent edges, two at a time, and then add up the results. The following lemma however suggests a more efficient approach.

Lemma 4. Consider a bipartite graph $G=(U \cup W, E)$ with girth $g$, and a node $v \in U$. Initiate the message-passing algorithm by passing 1 on all the edges connected to nodes $u \in U, u \neq v$, while passing $d_{v}$ different monomials, say $X_{1}, X_{2}, \ldots, X_{d_{v}}$, along the edges connected to $v: e_{1}, \ldots, e_{d_{v}}$, respectively. For $k \leq g-1$, we then have

$$
N_{2 k}^{v}=\sum_{j=1}^{d_{v}} \operatorname{ex}\left(m_{E, v \leftarrow}^{(k)} \underset{e_{j}}{(k)}\right) / 2,
$$

where $N_{2 k}^{v}$ is the number of $2 k$-cycles containing $v$.

Proof. At iteration $k \leq g-1$, consider the message received by $v$ along $e_{j}, j=$ $1, \ldots, d_{v}$, excluding the variable $X_{j}$. In this extrinsic message $m_{E, v \leftarrow}^{(k)}$, the power of variable $X_{i}, i \neq j$, is $N_{2 k}^{v ; e_{i}, e_{j}}$. We therefore have

$$
\operatorname{ex}\left(m_{E, v \leftarrow}^{(k)}{ }_{\substack{e_{j} \\ j}}\right)=\sum_{\substack{i=1 \\ i \neq j}}^{d_{v}} N_{2 k}^{v ; e_{i}, e_{j}} .
$$

This combined with

$$
N_{2 k}^{v}=\frac{1}{2} \sum_{j=1}^{d_{v}} \sum_{\substack{i=1 \\ i \neq j}}^{d_{v}} N_{2 k}^{v ; e_{i}, e_{j}},
$$


completes the proof.

In Lemma 4 , at iteration $k, k<g / 2$, node $v$ will only receive 1 along all its edges, indicating there are no cycles of length $g-2$ or smaller containing $v$.

It is worth noting that the message-passing algorithm can be simplified by allowing node $v$ to always pass 1 after the first iteration. This is demonstrated in the following example.

\subsubsection{A Simple Example}

Here, we illustrate the proposed method by a simple example. Consider the bipartite graph $G$ shown in Figure 3.4(a), where the nodes in $U$ and $W$ are represented by hollow and full circles, respectively.

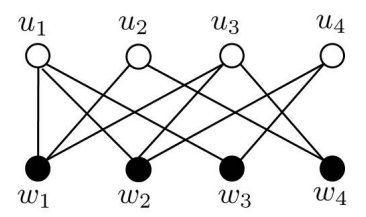

(a)

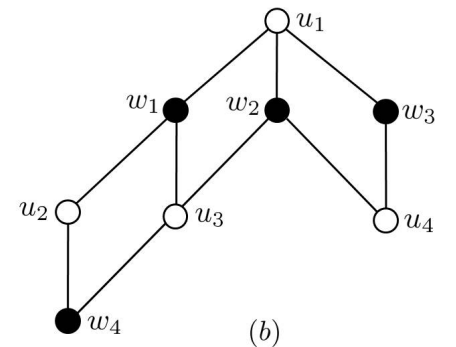

(b)

Figure 3.4: Bipartite graph of the example in Section III.C: a) $G$, b) $G$ unwound from node $u_{1}$.

Suppose that we are interested in counting short cycles containing node $u_{1}$. For the simplicity of presentation, as shown in Figure 3.4(b), we can unwind the graph $G$ from node $u_{1}$. It is easy to see from Figure 3.4(b) that the girth of $G$ is 4 . Using the proposed method, we can thus count cycles of length up to $2 g-2=6$. The message-passing algorithm is illustrated in Figures 3.5(a)-(f):

(a) At $t=0$, the algorithm is initiated by node $u_{1}$ passing messages $X_{1}, X_{2}$, and $X_{3}$ along its 3 edges. All the other messages sent by nodes $u_{2}, u_{3}$ and $u_{4}$ along their edges are equal to 1 , and not shown. [Equivalently, in the vector representation, the initial messages of node $u_{1}$ are vectors $(1,0,0),(0,1,0)$ and $(0,0,1)$, while all the other messages are $(0,0,0)$.]

(b) At $t=1$, only the nodes in $W$ are active. The corresponding (non-one) messages are shown in Figure 3.5(b). Note that in this iteration $(\ell=1)$, all the 

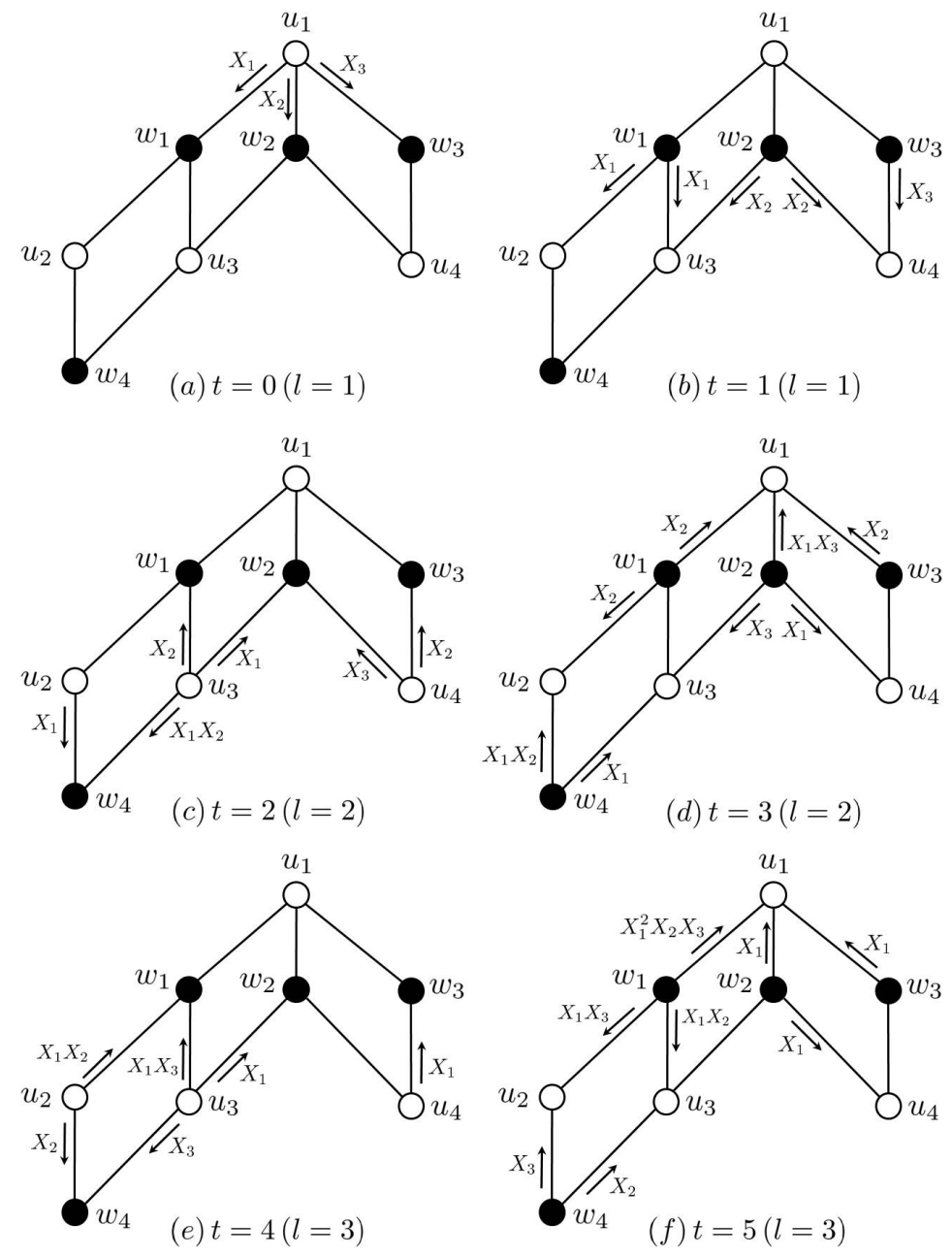

Figure 3.5: Message passing of the proposed algorithm for three iterations in the graph of Figure 3.4.

incoming messages to node $u_{1}$ are equal to one.

(c) At $t=2$, nodes in $U$ are active. They all pass extrinsic messages using multiplication. For example, $m_{u_{3} \rightarrow w_{4}}^{(2)}=m_{w_{1} \rightarrow u_{3}}^{(1)} \times m_{w_{2} \rightarrow u_{3}}^{(1)}=X_{1} X_{2}$. [In the vector representation, $m_{u_{3} \rightarrow w_{4}}^{(2)}=(1,0,0)+(0,1,0)=(1,1,0)$.]

(d) At $t=3(\ell=2)$, for the first time node $u_{1}$ receives non-one messages, an indication that there is at least one cycle of length $2 \ell=4$ containing $u_{1}$. Using (3.2), we obtain $N_{4}^{u_{1}}=(1+2+1) / 2=2$.

(e) At $t=4$, the nodes in $U$ are active and pass messages.

(f) At $t=5(\ell=3)$, nodes in $W$ are active. Again in this iteration, node $u_{1}$ receives non-one messages, an indication that it belongs to at least one 6-cycle. Using (3.2), we have $N_{6}^{u_{1}}=(2+1+1) / 2=2$. 


\subsection{Proposed Message-Passing Algorithm}

\subsubsection{Pseudo Code}

To count the short cycles of a certain length $2 k$ in the whole graph $G=(U \cup W, E)$, one can apply the proposed algorithm described in the previous section to every node in one of the node partitions, $U$ or $W$, and then add up the results for each cycle length. In this case, for each cycle length, the result should be divided by $k$ as every cycle is counted $k$ times:

$$
N_{2 k}=\left(\sum_{u \in U} N_{2 k}^{u}\right) / k=\left(\sum_{w \in W} N_{2 k}^{w}\right) / k, \quad \frac{g}{2} \leq k \leq g-1 .
$$

To simplify the algorithm and to avoid the $k$-fold counting repetition, we can deactivate a node as soon as its cycles are counted. This would be equivalent to removing the node and all its adjacent edges from the graph. Moreover, the algorithm can be further simplified by only activating nodes that have at least one non-one incoming message. Based on these simplifications, the proposed algorithm has the pseudo code provided in Algorithm 1.

Algorithm 1: Proposed Message-Passing Algorithm for Counting Short Cycles

for $k=1: g-1$ do

counter $(k)=0$

end for

for $i=1: n$ do

\section{Initialization}

$l=1$

for $w_{j} \in N\left(u_{i}\right)$ do

$$
\begin{aligned}
& m_{u_{i} \rightarrow w_{j}}^{(0)}=X_{l} \\
& l=l+1
\end{aligned}
$$

\section{end for}

for $i^{\prime}=i+1: n$ do

for $w_{j} \in N\left(u_{i^{\prime}}\right)$ do

$$
m_{u_{i^{\prime}} \rightarrow w_{j}}^{(0)}=1
$$




\section{end for}

\section{end for}

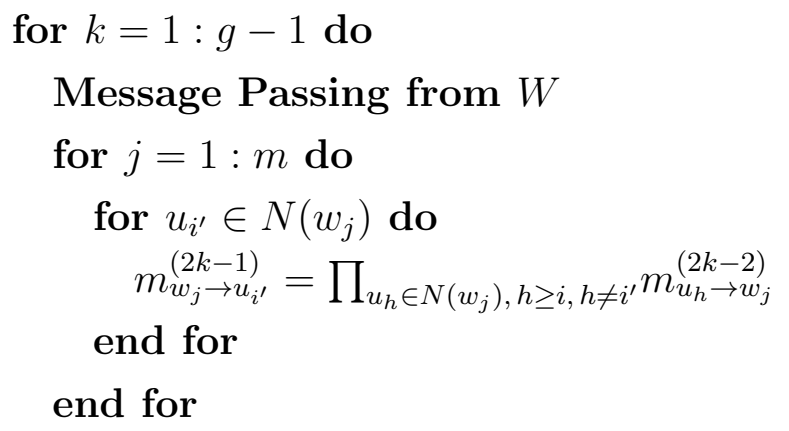

\section{Counting Cycles}

local-counter $(k)=\sum_{w_{j} \in N\left(u_{i}\right)} \operatorname{ex}\left(m_{E,\left(w_{j} \rightarrow u_{i}\right)}^{(2 k-1)}\right)$

\section{Message Passing from $U$}

for $i^{\prime}=i+1: n$ do

for $w_{j} \in N\left(u_{i^{\prime}}\right)$ do

$$
m_{u_{i^{\prime}} \rightarrow w_{j}}^{(2 k)}=\prod_{w_{h} \in N\left(u_{i^{\prime}}\right), h \neq j} m_{w_{h} \rightarrow u_{i^{\prime}}}^{(2 k-1)}
$$

end for

\section{end for}

\section{end for}

for $k=1: g-1$ do

counter $(k)=$ counter $(k)+$ local-counter $(k) / 2$

end for

end for

Algorithm 1 is initiated from $U$. Similarly, it can be initiated from $W$. Nodes in $U$ are indexed by $i=1, \ldots, n$, and notation $m_{E,\left(w_{j} \rightarrow u_{i}\right)}$ is used to denote the incoming message from node $w_{j}$ to node $u_{i}$ excluding the initial variable passed from $u_{i}$ to $w_{j}$. Notation $N(u)$ is used for the nodes adjacent to $u$ (neighbors of $u$ ).

Here, we have implicitly assumed that the girth $g$ of the graph is known. In the following subsection, we discuss a modification of the algorithm that can compute $g$ 
and $N_{g}$.

\subsubsection{Parallel Implementation}

The algorithm presented in the previous subsection is based on sequentially going through the nodes in one of the two partitions in the graph. To speed up the counting process and at the expense of larger memory usage, one can run a parallel version of the algorithm in which all the nodes in one partition are initialized simultaneously. This is explained in Figure 3.6(a) for the graph of Figure 3.4.

The parallel implementation, just described, can also be used to compute $g$ and $N_{g}$. To see this, note that in the parallel implementation, none of the nodes in the initiating partition will receive a copy of its initial messages before iteration $g / 2$. At iteration $g / 2$, the nodes which are contained in the shortest cycles will receive copies of their initial messages and all such copies are received along the edges whose initial messages differ from the received messages. This means that all the received copies represent true $g$-cycles. Therefore to compute $g$ and $N_{g}$, one does not need to distinguish among the initial messages of a node. The initialization in this case is explained in Figure 3.6(b) for the graph of Figure 3.4. In this setup, if the first iteration in which at least one of the nodes receives a non-one message is iteration $k$, then $g=2 k$, and the number of $g$-cycles is equal to the total number of received non-one messages by all the nodes divided by $2 k$.

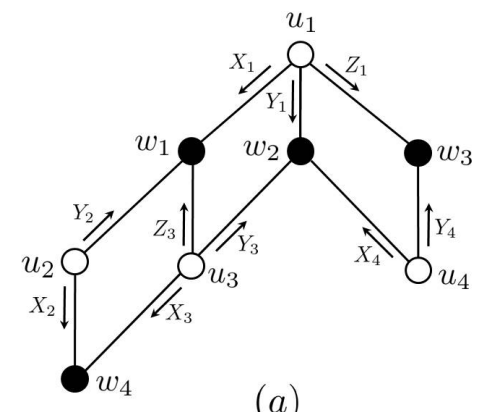

(a)

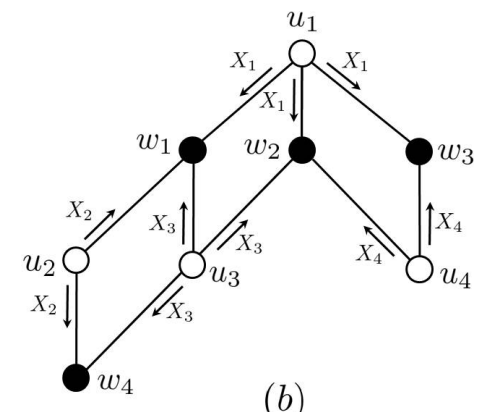

(b)

Figure 3.6: Initial message-passing in parallel implementations: a) counting short cycles of length $2 k, g / 2 \leq k \leq g-1$, b) calculating $g$ and $N_{g}$. 


\subsection{Complexity of the Proposed Algorithm}

\subsubsection{Computational Complexity}

In the following, we arbitrarily assume that the algorithm is initiated from the node set $U$. We consider a sequential implementation, where the nodes in $U$ are processed one at a time. We also consider the vector representation of messages and first derive the complexity for a regular graph. We then generalize the results to irregular graphs. For a regular graph $G=(U \cup W, E)$, starting from a node $u \in U$, there are $d_{u}$ initial messages, each represented by a unit vector of length $d_{u}$. All the subsequent messages are also vectors of length $d_{u}$. To calculate the messages at an active node $w \in W$, we first add all the incoming vectors to $w$, and then subtract from this, the incoming message along each adjacent edge to obtain the outgoing message along that edge. This requires $\left(2 d_{w}-1\right) d_{u}$ integer additions and subtractions. Similarly, for each active node $u \in U$, we need $\left(2 d_{u}-1\right) d_{u}$ integer additions and subtractions to obtain the outgoing messages. Considering that in even and odd time instances, the number of active nodes are upper bounded by $n$ and $m$, respectively, the number of operations per iteration is of order $n d_{u}^{2}+m d_{u} d_{v}=|E| d_{u}$. Since the algorithm needs to perform $g-1$ iterations, the complexity of the algorithm for each node $u \in U$ is of order $g n d_{u}^{2}+g m d_{u} d_{v}=g|E| d_{u}$. The total complexity is thus of order

$$
g n^{2} d_{u}^{2}+g n m d_{u} d_{v}=g n^{2} d_{u}^{2}=g|E|^{2} .
$$

It is easy to see that the same complexity order also applies to irregular bipartite graphs.

In the above discussions, it is implicitly assumed that the girth of the graph is known a priori. Since the computational complexity of finding the girth is at most $O\left(n^{2}\right)$, e.g., based on the algorithm of [72], ${ }^{1}$ the extra complexity for computing the girth is negligible compared to the rest of the computations.

\footnotetext{
${ }^{1}$ It is easy to see that if we use the algorithm proposed in Section IV.B to compute $g$, the complexity is of order $g n^{2} d_{u}$, which is in general larger than that of [72]. The algorithm of [72] however only finds $g$, while the proposed algorithm also computes $N_{g}$.
} 


\subsubsection{Memory Requirements}

For each edge of the bipartite graph, we need two memory locations to store the message vectors in both directions. For a regular graph, since each vector has $d_{u}$ elements, the total number of memory locations, each storing an integer number, is of order $2 d_{u}|E|=2 n d_{u}^{2}$. For an irregular graph, the storage complexity is of order $d_{\max }|E|$, where $d_{\max }$ is the maximum node degree in $U$ or $W$, depending on which side initiates the algorithm.

\subsection{Comparison with Existing Literature}

\subsubsection{Comparison with the Algorithm of [36]}

First, it is important to note that while the algorithm of [36] is limited to bipartite graphs, the proposed algorithm is capable of counting short cycles in a general (nonbipartite) graph. For bipartite graphs, the algorithm of [36] counts cycles of length $g, g+2, g+4$, while the proposed algorithm counts cycles of length $g, g+2, \ldots, 2 g-2$. The coverage of the proposed algorithm is thus at least as much as the algorithm of [36] for graphs with $g \geq 6$. It should be noted that the Tanner graphs of almost all good LDPC codes have $g \geq 6$.

The computational complexity of the algorithm of [36] is of order $g n^{3}$, where $n=\max (|U|,|W|)$. The complexity of the proposed algorithm is of order $g|E|^{2}$. One can thus see that for sparse graphs with $|E|$ growing slower than $n^{3 / 2}$, the complexity of the proposed algorithm is less than that of the algorithm in [36]. Moreover the computations in the algorithm presented here are simple integer additions and subtractions, while in [36] the operations are mainly high-precision multiplications.

In terms of memory requirements, the algorithm of [36] requires at most $11\left(n^{2}+\right.$ $\left.m^{2}\right)+21 n m$ high bit-width (64-bit integer) storage locations, which is of order $O\left(n^{2}\right)$. The proposed algorithm on the other hand requires $2 d_{\max }|E|$ memory locations, which for sparse graphs can be much smaller than what is needed for the algorithm of [36]. Moreover, the maximum size of memory locations for the proposed algorithm, which is proportional to the number of cycles, is usually much less than 64 bits. 


\subsubsection{Relationship and Comparison with Matrix Multiplica- tion Techniques}

A natural approach to counting the number of $k$-cycles in a graph $G$ is to use the $k$ th power of the directed edge matrix $A_{e}$ of $G$. In general, $\operatorname{tr}\left(A_{e}^{k}\right)$ is equal to the total number of tbc walks of length $k$ in $G$. Now considering that each tbc walk of length $k$ appears $k$ times in the counting process (equivalent tbc walks are counted multiple times), and that each tbc walk is counted twice for the two directions, one can see that the total number of undirected non-equivalent tbc walks of length $k$ is $\operatorname{tr}\left(A_{e}^{k}\right) / 2 k$. Based on Lemma 2, all the tbc walks of length $k<2 g$ are cycles. We thus have

$$
N_{k}=\operatorname{tr}\left(A_{e}^{k}\right) / 2 k, \text { for } k<2 g \text {. }
$$

\section{Complexity of calculating $A_{e}^{k}$}

There are a number of methods for calculating the powers of a matrix. These methods have different complexities and their relative performance may depend on the sparsity level of the matrix. For the Tanner graph of LDPC codes, for example, the matrix $A_{e}$ has only $\sum_{i=1}^{n} d_{u_{i}}^{2}+\sum_{i=1}^{m} d_{w_{i}}^{2}-2|E|$ nonzero (one) elements. For the case of a regular LDPC code, this simplifies to $|E|\left(d_{u}+d_{w}-2\right)$ nonzero elements. This implies that for the Tanner graph of LDPC codes, the matrix $A_{e}$ is sparse.

To compute $A_{e}^{k}$, one can use the iterative method: $A_{e}^{k}=A_{e}^{k-1} A_{e}$. Another approach is to use successive squares method: $A_{e}^{k}=\prod_{l} A_{e}^{2^{l}}$, where $\sum_{l} 2^{l}$ represents the binary expansion of $k$. While the successive squares method is generally more efficient than the iterative method, the latter can be more efficient in the case that $A_{e}$ is sparse. The reason is that the powers of a sparse matrix may not be sparse, and thus the general complexity of the successive squares method is determined by the complexity of dense matrix multiplication. This is while, in the case of the iterative method, in each iteration, at least one of the matrices (i.e., $A_{e}$ ) is sparse. This property can be used to reduce the computational complexity of the matrix multiplication.

Consider two integer $N \times N$ matrices $A$ and $B$. The complexity of the straightforward calculation of $C=A \cdot B$ is $O\left(N^{3}\right)$. One of the first algorithms proposed for faster matrix multiplication is by Strassen [98] and has a complexity of $O\left(N^{2.81}\right)$. Currently, the fastest known algorithm for matrix multiplication is the CoppersmithWinograd algorithm [17] which has an asymptotic complexity of $O\left(N^{2.376}\right)$. This 
algorithm however, cannot utilize the sparsity (even if any or both of the two matrices is sparse), and its complexity remains unchanged even if the multiplied matrices are extremely sparse [127]. Moreover, in the Coppersmith-Winograd algorithm, the complexity $O\left(N^{2.376}\right)$ is only achieved when the size of the matrices tends to infinity. More recently, Yuster and Zwick [127] presented a method for fast sparse matrix multiplication with the asymptotic complexity of $O\left(M^{0.7} N^{1.2}+N^{2+o(1)}\right)$, where $M$ is the number of nonzero elements in the less sparse matrix among the two. This complexity result not only is asymptotic and applies only to very large matrices, but also requires both matrices to be sparse. One however should note that even the product of two very sparse matrices can be very dense, and thus the algorithm of [127] would not be applicable to the case of finding the $k$-th power of a sparse matrix.

For the case where only one of $A$ or $B$ is sparse, one can use the straightforward method of calculating the product $C=A \times B$ through: $c_{i j}=\sum_{k=1}^{N} a_{i k} b_{k j}$. It is easy to show that in this case, the complexity of multiplication is of order $M N$, where $M$ is the number of nonzero elements in the sparser matrix between $A$ and $B$. Applying this result to the calculation of $A_{e}^{k}$ by the iterative method, one can see that the computational complexity is of order $k M N$. For the Tanner graph of regular LDPC codes, this reduces to $k|E|^{2}\left(d_{u}+d_{w}\right)$, which implies that the complexity of counting the cycles of length $k, g \leq k \leq 2 g-2$, using (3.4) is of order $g|E|^{2}\left(d_{u}+d_{w}\right)$ or $g n^{2} d_{u}^{3}+g n^{2} d_{u}^{2} d_{w}$. It is worth noting that since all the nonzero elements of $A_{e}$ are ones, all the operations in computing $A_{e}^{k}$ are simple additions. The storage complexity of this method is dominated by the required memory to store $A_{e}^{k-1}$. Since $A_{e}^{k-1}$ can be dense, the storage complexity of the method is of order $|E|^{2}$ or $n^{2} d_{u}^{2}$.

The comparison of the computational complexity and memory requirements of the proposed algorithm, which are of orders $g|E|^{2}$ and $d_{\max }|E|$, respectively, with those of the above matrix multiplication method (naive approach), which are of orders $g|E|^{2}\left(d_{v}+d_{w}\right)$ and $|E|^{2}$, respectively, shows the superiority of the proposed method.

\section{Efficient calculation of $N_{k}$ using matrix multiplication}

Consider the directed edge matrix $A_{e}$ corresponding to a graph $G=(V, E)$. We construct a $|V| \times 2|E|$ matrix $B$ from $A_{e}$ by adding the rows of $A_{e}$ that correspond to the edges emanating from each node $v$ of $G$ for every $v \in V$, and removing all the other rows. Note that these rows have no overlap in the nonzero locations. We then 
multiply $B$ by $A_{e}$ from the right $k-1$ times to obtain

$$
B^{\prime}=\left(\cdots\left(\left(B \cdot A_{e}\right) \cdot A_{e}\right) \cdots \cdot A_{e}\right) .
$$

We then derive a $|V| \times|V|$ matrix $B^{\prime \prime}$ from $B^{\prime}$ by adding up the columns of $B^{\prime}$ that correspond to the edges emanating from each node $v$ of $G$ for every $v \in V$ (and removing all the other columns), and then dividing the $i$ th diagonal element of the resulting matrix by $d_{i}-1$, where $d_{i}$ is the degree of the $i$ th node in $G$. The complexity of obtaining $B^{\prime \prime}$ from $B^{\prime}$ is of order $|V||E|$. Based on (3.4), it is then easy to see that

$$
N_{k}=\operatorname{tr}\left(B^{\prime \prime}\right) / 2 k, \text { for } k<2 g \text {. }
$$

The complexity of calculating $N_{k}$ using (3.5) and (3.6) is of order $k|V| M$, where $M$ is the number of nonzero elements in $A_{e}$. For the Tanner graph of regular LDPC codes, this reduces to $k|E|^{2}\left(d_{u}+d_{w}\right)^{2} /\left(d_{u} d_{w}\right)$, which implies that the complexity of counting the cycles of length $k, g \leq k \leq 2 g-2$, for the Tanner graph of a regular LDPC code of a given rate $r=1-d_{u} / d_{w}$ using (3.6) is of order $g|E|^{2}$, which is similar to that of the proposed message-passing algorithm. However, it should be noted that the memory requirement of the matrix multiplication approach is of order $|V||E|$, which for example for a regular LDPC code reduces to $|E|^{2} / d_{u}$ which is larger than that of the proposed algorithm.

\section{Message-passing interpretation of matrix multiplication algorithms}

Consider a bipartite graph representation of $A_{e}$, where one set of nodes, say, those on the left, represent the rows of $A_{e}$, and the other set of nodes (those on the right) represent the columns of $A_{e}$. An edge is connected between node $i$ on the left and node $j$ on the right if and only if the $(i j)$ th element of $A_{e}$ is nonzero. Suppose that we connect $k$ such graphs together in sequence, such that the right nodes of the $i$ th graph coincides with the left nodes of the $(i+1)$ th graph, for $i=1, \ldots, k-1$. We call the resulting graph a trellis diagram, or a trellis in brief, following the nomenclature used in coding. Both the naive and the efficient calculations of $N_{k}$ can be described by a message-passing algorithm over this trellis.

The message-passing algorithm is started by assigning initial values to the leftmost nodes of the trellis. These nodes then pass their initial messages along their outgoing edges to the adjacent set of nodes. The algorithm is then continued by the 
messages being passed forward (left to right) in the trellis, one trellis section at a time, with each node adding up the messages it receives along its incoming edges on the left and passing the result along all its outgoing edges on the right. The algorithm will end by all the right-most nodes receiving messages along their incoming edges and passing out the sum of those messages as their output.

If the message-passing algorithm is initiated by zero values on all the left-most nodes in the trellis except for node $i$, which is assigned the value 1 , then the set of outputs will be the $i$ th row of matrix $A_{e}^{k}$. If the values of the input trellis nodes corresponding to the rows of $A_{e}$ that represent the outgoing edges of node $v$ of $G$ are set to one with the rest of the input values equal to zero, then the set of outputs will be equal to the row of $B^{\prime}$ in (3.5) which corresponds to $v$.

As a final note, one should realize that although the message-passing algorithms over the trellis diagram and the original graph (for counting the short cycles) have both essentially the same complexity, the graph representation of the latter (original graph) is simpler than that of the former (a trellis section). (Note that for example, for a regular $\left(d_{u}, d_{w}\right)$ LDPC code, one section of the trellis diagram has $|E|\left(d_{u}+d_{w}-2\right)$ edges, while the original Tanner graph has only $|E|$ edges.)

\subsection{Numerical Results}

In this section, we present numerical results obtained by applying the proposed algorithm to Tanner graphs of LDPC codes. We consider four rate- $1 / 2$ codes from [132]. Codes A and B are listed in [132] as PEGirReg504x1008 and PEGReg504x1008, respectively. Both codes are constructed using the Progressive Edge Growth (PEG) method of [39], and have $n=1008$ and $m=504$. Code A is irregular while Code $\mathrm{B}$ is regular. Codes C and D are MacKay's codes 8000.4000.3.483 and 10000.10000.3.631, respectively. They are both regular with $d_{u}=3$ and $d_{w}=6$. For Code C, $n=8000$ and $m=4000$, while these parameters for Code D are 20,000 and 10,000, respectively. The number of short cycles in the Tanner graphs of these codes is listed in Table 3.1. Codes A, C and D have girth 6 and the proposed algorithm, similar to the algorithm of [36], can compute $N_{6}, N_{8}$ and $N_{10}$. Code B however has girth 8, and while the algorithm of [36] can only compute $N_{8}, N_{10}$ and $N_{12}$, the proposed algorithm can also compute $N_{14}$. 
Table 3.1: Number of Short Cycles in the Tanner Graphs of Four Rate-1/2 LDPC Codes

\begin{tabular}{|l|c|c|c|c|}
\hline & Code A & Code B & Code C & Code D \\
\hline \hline$N_{6}$ & 11538 & 0 & 179 & 161 \\
$N_{8}$ & 408657 & 2 & 1218 & 1260 \\
$N_{10}$ & 13110235 & 11238 & 9989 & 10051 \\
$N_{12}$ & - & 91101 & - & - \\
$N_{14}$ & - & 748343 & - & - \\
\hline
\end{tabular}

Tables 3.2 and 3.3 show the running time and memory requirements of the proposed algorithm and the algorithm of $[36],{ }^{2}$ respectively. Both algorithms were run on the same machine with a 2.2-GHz CPU and $8 \mathrm{~GB}$ of RAM. As can be seen, the proposed algorithm is consistently faster than the algorithm of [36] and requires significantly less memory for larger graphs. In fact, for Code D, the algorithm of [36] ran out of memory and was not able to find the results. ${ }^{3}$

Table 3.2: CPU Time and Memory Requirements for the Proposed Algorithm

\begin{tabular}{|c|c|c|c|}
\hline & $\begin{array}{c}\text { CPU Time } \\
(\mathbf{S})\end{array}$ & $\begin{array}{c}\text { Max Memory } \\
\text { (MB) }\end{array}$ & $\begin{array}{c}\text { Max Swap } \\
\text { (MB) }\end{array}$ \\
\hline \hline Code A & 5.3 & 0.36 & 3.3 \\
Code B & 3 & 0.36 & 2.8 \\
Code C & 155 & 13 & 157 \\
Code D & 1127 & 13 & 157 \\
\hline
\end{tabular}

As another experiment, we randomly generate six parity-check matrices for each of the following three rate- $1 / 2$ LDPC code ensembles: $\left(d_{u}, d_{w}\right)=(3,6),(4,8),(5,10)$. The lengths for each degree distribution are: $n=200,500,1000,5000,10,000$ and 20,000. In the generation of the parity-check matrices, 4-cycles are avoided. The proposed algorithm is then used to count the short cycles of each parity-check matrix. The results, which are reported in Table 3.4, show that while there is a large difference

\footnotetext{
${ }^{2}$ To implement the algorithm of [36], we used the authors' code in [133].

${ }^{3}$ The reason that the memory requirements of Code $\mathrm{C}$ and Code $\mathrm{D}$ in Table 3.2 are similar is that the software used for reporting the storage requirements quantized the results to specific values.
} 
Table 3.3: CPU Time and Memory Requirements for the Algorithm of [36]

\begin{tabular}{|c|c|c|c|}
\hline & $\begin{array}{c}\text { CPU Time } \\
(\mathbf{S})\end{array}$ & $\begin{array}{c}\text { Max Memory } \\
\text { (MB) }\end{array}$ & $\begin{array}{c}\text { Max Swap } \\
\text { (MB) }\end{array}$ \\
\hline \hline Code A & 10.3 & 1.5 & 35 \\
Code B & 16.6 & 1.5 & 35 \\
Code C & 4965 & 7839 & 14195 \\
Code D & - & - & - \\
\hline
\end{tabular}

between the short cycle distribution of different degree distributions, the changes with respect to the block length for the same degree distribution are negligible. Also we make the observation that as expected for the same block length as the degrees increase the multiplicity of short cycles of each length increases. This would imply that the complexity of the algorithms which are based on the enumeration of short cycles in a Tanner graph is rather independent of the block length. ${ }^{4}$

\footnotetext{
${ }^{4}$ It is worth mentioning that it is proved in [73] that for random regular bipartite graphs with $d_{u}=d_{w}=d$, as the number of nodes tends to infinity, the distribution of short cycles of different length $c_{i}$ tends to independent Poisson distributions with average $\mu_{i}=(d-1)^{c_{i}} / c_{i}$. To the best of our knowledge, however, no generalization of this result is available for bipartite graphs with $d_{u} \neq d_{w}$.
} 
Table 3.4: Distribution of Short Cycles in the Tanner Graphs of Rate- $1 / 2$ Random Regular LDPC Codes with Different Degree Distributions and Different Block Lengths

\begin{tabular}{|c|l|l|l|l|l|l|l|}
\hline \multirow{2}{*}{$\begin{array}{c}\text { Degree } \\
\text { Dist. }\end{array}$} & \multirow{2}{*}{$\begin{array}{l}\text { Short Cycle } \\
\text { Dist. }\end{array}$} & \multicolumn{7}{|c|}{ Code Lengths } \\
\cline { 3 - 8 } & $\mathbf{2 0 0}$ & $\mathbf{5 0 0}$ & $\mathbf{1 0 0 0}$ & $\mathbf{5 0 0 0}$ & $\mathbf{1 0 0 0 0}$ & $\mathbf{2 0 0 0 0}$ \\
\hline \hline \multirow{3}{*}{$(3,6)$} & $N_{6}$ & 171 & 167 & 181 & 156 & 166 & 148 \\
& $N_{8}$ & 1265 & 1239 & 1226 & 1235 & 1253 & 1285 \\
& $N_{10}$ & 10069 & 10110 & 9939 & 9982 & 9858 & 9974 \\
\hline \multirow{3}{*}{$(4,8)$} & $N_{6}$ & 1636 & 1611 & 1584 & 1562 & 1537 & 1572 \\
& $N_{8}$ & 25005 & 24419 & 24379 & 24363 & 24529 & 24557 \\
& $N_{10}$ & 409335 & 409373 & 408595 & 407958 & 408246 & 409051 \\
\hline \multirow{2}{*}{$(5,10)$} & $N_{6}$ & 8626 & 8064 & 8055 & 7978 & 7858 & 7926 \\
& $N_{8}$ & 213639 & 212484 & 210767 & 210153 & 209614 & 210159 \\
& $N_{10}$ & 6052158 & 6054661 & 6049148 & 6043400 & 6049583 & 6043704 \\
\hline
\end{tabular}




\section{Chapter 4}

\section{Counting Short Cycles of Quasi Cyclic Protograph LDPC Codes}

In this chapter, our focus is on the short cycle distribution of the Tanner graphs of QC protograph LDPC codes. QC protograph LDPC codes are an important class of LDPC codes adopted in many standards. This is mainly because these codes lend themselves to low complexity encoder and decoder architectures, which are implementation-friendly. Using the structure of the codes, we first propose an efficient algorithm (less complex than that of the algorithm proposed in the previous chapter) for counting the short cycles of the corresponding Tanner graphs. We then use the proposed algorithm to investigate the short cycle distribution of a few ensembles of QC protograph LDPC codes and demonstrate that, on average, they have a superior short cycle and girth distribution compared to similar randomly constructed codes.

\subsection{Proposed Method}

The directed edge matrix of a graph can be used to count the tbc walks of different length in the graph as shown in (2.1). It was shown in Chapter 3 that the set of tbc walks of length less than $2 g$ coincides with the set of cycles of the same sizes in a graph with girth $g$. We thus have $N_{k}=\operatorname{tr}\left(A_{e}^{k}\right) / 2 k, k<2 g$, where $N_{k}$ denotes the number of cycles of length $k$. It is also known that $\operatorname{tr}\left(A_{e}^{k}\right)=\sum_{i} \lambda_{i}^{k}$, where $\lambda_{i}$ 's are the eigenvalues of $A_{e}$. Combining these together, we have

$$
N_{k}=\sum_{i} \lambda_{i}^{k} /(2 k), k<2 g .
$$


To use (4.1) for counting the short (shorter than $2 g$ ) cycles of a QC protograph LDPC code, we need to calculate the eigenvalues of $A_{e}$. This has a complexity of $O\left(|E|^{3}\right)[108]^{1}$ which can be prohibitively high for (large) Tanner graphs of many practical LDPC codes. To find a more efficient algorithm for calculating $\lambda_{i}$ 's, we study the structure of $A_{e}$ for Tanner graphs of QC protograph LDPC codes.

We first note that for any bipartite graph (including Tanner graphs) if we label all the directed edges that have their head on one side of the graph with labels $1, \ldots,|E|$, and all the directed edges with the opposite direction by labels $|E|+1, \ldots, 2|E|$, then we will have

$$
A_{e}=\left[\begin{array}{ll}
0 & B \\
C & 0
\end{array}\right],
$$

where $B$ and $C$ are symmetric $|E| \times|E|$ matrices with zero diagonal elements.

The following theorem helps with simplifying the calculation of $\lambda_{i}$ 's by taking advantage of the structure of (4.2).

Theorem 1. Let $A_{e}$ be a matrix of form (4.2). Then the eigenvalues of $A_{e}$ are symmetric with respect to the origin, i.e., if $\lambda$ is an eigenvalue, so is $-\lambda$. Moreover, $\lambda$ is an eigenvalue of $A_{e}^{2}$ if and only if $\pm(\lambda)^{1 / 2}$ are eigenvalues of $A_{e}$.

Proof. The proof is similar to that of Theorem 8.6.9 of [116], and is thus omitted.

One can use Theorem 1 to find the eigenvalues of $A_{e}$ based on those of $A_{e}^{2}$. This reduces the complexity because of the form of $A_{e}^{2}$ :

$$
A_{e}^{2}=\left[\begin{array}{cc}
B C & 0 \\
0 & C B
\end{array}\right]
$$

which implies that the eigenvalues of $A_{e}^{2}$ are given by the union of the eigenvalues of matrices $B C$ and $C B$. As the nonzero eigenvalues of these matrices are the same, to find the eigenvalues of $A_{e}^{2}$, it is enough to find the eigenvalues of $B C$, which is a smaller matrix than $A_{e}^{2}$.

Consider the directed edge matrix $A_{e, b}$ of the base graph $G_{b}=\left(U_{b} \cup W_{b}, E_{b}\right)$, which has the form (4.2) with submatrices $B_{b}$ and $C_{b}$. Suppose that $A_{e, b}$ is obtained

\footnotetext{
${ }^{1}$ In general, the complexity of finding the eigenvalues of an $n \times n$ matrix is $O\left(n^{3}\right)$.
} 
by labeling the incoming edges to the variable nodes from 1 to $\left|E_{b}\right|$ by going through the variable nodes one by one and labeling all the edges of each variable node in sequence followed by the edges of the next variable node and so on. Now consider the following labeling of the incoming edges of the variable nodes of the lifted graph $G$ from 1 to $|E|$. We go through the $N$ copies of $G_{b}$ in $G$ one by one, and for each copy, we label all the incoming edges of its variable nodes in the same sequence that we labeled the edges of $G_{b}$ when constructing $A_{e, b}$. The labeling of edges continues in sequence by going from one copy of $G_{b}$ to the next. Based on this labeling, one can see that the submatrices $B$ and $C$ of the directed edge matrix of the protograph code, $A_{e}$ in (4.2), are related to $B_{b}$ and $C_{b}$ by

$$
B=\operatorname{Bcirc}\left(B_{b}, 0, \ldots, 0\right),
$$

and

$$
C=\operatorname{Bcirc}\left(C_{0}, \ldots, C_{N-1}\right),
$$

respectively, where $\operatorname{Bcirc}\left(C_{0}, \ldots, C_{N-1}\right)$ is a block circulant matrix, consisting of $N \times N$ blocks, each of size $\left|E_{b}\right| \times\left|E_{b}\right|$, with the first block row equal to $\left(C_{0}, \ldots, C_{N-1}\right)$ and each subsequent block row equal to the circular shift of the previous block row to the right by one block. For the sub-blocks of $C$, we have $\sum_{i} C_{i}=C_{b}$. Combining (4.4) and (4.5), we obtain

$$
B C=\left[\begin{array}{cccc}
B_{b} C_{0} & B_{b} C_{1} & \cdots & B_{b} C_{N-1} \\
B_{b} C_{N-1} & B_{b} C_{0} & \cdots & B_{b} C_{N-2} \\
\vdots & \vdots & \ddots & \vdots \\
B_{b} C_{1} & B_{b} C_{2} & \cdots & B_{b} C_{0}
\end{array}\right]
$$

which also has a block circulant structure. We then use the following theorem to simplify the calculation of the eigenvalues of (4.6).

Theorem 2 ( [104]). Let $B=B \operatorname{Birc}\left(b_{0}, b_{1}, \ldots, b_{r-1}\right)$ be a block circulant matrix in $\mathbb{C}^{r L \times r L}$. Then the eigenvalues of $B$ are given by the union of the eigenvalues of the $L \times L$ matrices $H(\rho)=b_{0}+b_{1} \rho+\cdots+b_{r-1} \rho^{r-1}$, where $\rho$ is a complex rth root of unity given by $\rho=\exp (i 2 \pi s / r), 0 \leq s \leq r-1$. 
Based on Theorem 2, to find the eigenvalues of (4.6), one needs to find the eigenvalues of $N$ matrices, each of size $\left|E_{b}\right| \times\left|E_{b}\right|$. This reduces the complexity from $O\left(N^{3}\left|E_{b}\right|^{3}\right)$ to $O\left(N\left|E_{b}\right|^{3}\right)$. Compared to the complexity $O\left(g|E|^{2}\right)=O\left(g N^{2}\left|E_{b}\right|^{2}\right)$ of Algorithm 1, the proposed algorithm is less complex if $g N$ grows faster than $\left|E_{b}\right|$. This would be the case for protograph codes with small base graph and large lifting degree.

In terms of memory requirements, for example for a regular LDPC code of variable degree $d_{u}$, the proposed method needs $N\left|E_{b}\right|^{2}$ memory locations to store (4.6). Algorithm 1, on the other hand, needs $2 d_{u}|E|$ locations, which would be less than that of the proposed method if $\left|U_{b}\right|>2$.

\subsection{Numerical Results}

The following results are obtained using MATLAB on a PC with $2.2 \mathrm{GHz}$ CPU and 8GB RAM.

Example 2. In this example, we consider the TSF $(155,64)$ code [100]. For this protograph code, the base graph is a fully connected $3 \times 5$ graph, and the lifting degree is $N=31$. The code has girth 8 and thus the proposed algorithm can count the number of short cycles up to the length $2 g-2=14: N_{8}=465, N_{10}=3720, N_{12}=22630$ and $N_{14}=156240$. It took less then 0.5 second for the proposed method to count these cycles.

Example 3. In this example, we still use the $3 \times 5$ fully connected graph as the base graph and lift it with degrees $N=400,1000$ and 2000, by selecting the cyclic permutations randomly. Table 4.1 shows the number of short cycles of the constructed codes. The results demonstrate that the number of shortest cycles reduces by increasing the lifting degree. For the three codes, the proposed method counted the cycles in 1.1, 6 and 18 seconds, respectively. This is while Algorithm 1 took 3.5, 20 and 119 seconds, respectively, to perform the same task. It is also worth noting that the algorithm of [36] took 126 and 1847 seconds for the first two codes, respectively, and ran out of memory and was unable to find the results for the third code. In the last column of Table 4.1, we have given the short cycle distribution of a randomly constructed $(3,5)$ regular Tanner graph in which 4-cycles are avoided. As can be seen, the short cycle distribution of this code is significantly inferior to that of the similar randomly 
constructed $Q C$ code. In particular, the former graph has $g=6$ while the girth of the latter is 12 .

Table 4.1: Number of Short Cycles for Different Liftings of the $3 \times 5$ Fully Connected Base Graph and the Short Cycle Distribution of a Random Graph with Similar Degree Distribution and Block Length 10,000

\begin{tabular}{|c|c|c|c||c|}
\hline \multirow{2}{*}{$\begin{array}{c}\text { Cycle } \\
\text { length }\end{array}$} & \multicolumn{3}{|c|}{ Lifting Degree } & \multirow{2}{*}{ Random } \\
\cline { 2 - 4 } & $N=400$ & $N=1000$ & $N=2000$ & Code \\
\hline \hline 6 & 0 & 0 & 0 & 106 \\
\hline 8 & 0 & 0 & 0 & 505 \\
\hline 10 & 2000 & 1000 & 0 & 3263 \\
\hline 12 & 33200 & 54000 & 98000 & 22199 \\
\hline 14 & 193200 & 275000 & 478000 & 149366 \\
\hline 16 & 1022200 & 1169000 & 1490000 & 1048092 \\
\hline 18 & 7143600 & 7251000 & 8282000 & 7437198 \\
\hline 20 & - & - & 68774400 & 53514806 \\
\hline 22 & - & - & 447084000 & 388717101 \\
\hline
\end{tabular}

Example 4. In this example, we consider the (irregular) LDPC codes adopted in IEEE 802.11 standard [41]. There are 12 codes in the standard with rates $R=1 / 2$, $2 / 3,3 / 4$ and $5 / 6$. For each rate, there are 3 codes, each with a different block length. The block lengths are $n=1944,1296$, and 648. For the block length $n=1944$, the codes are constructed based on base graphs with $\left(\left|W_{b}\right|,\left|U_{b}\right|\right)$ equal to $(12,24),(8,24)$, $(6,24)$ and $(4,24)$, respectively, and using a lifting of degree $N=81$. Table 4.2 shows the number of short cycles obtained by the proposed method for these codes. As can be seen in the table, the girth of all the protograph codes, except the code with rate $2 / 3$, is 6 , and thus, the method is able to find the number of short cycles of length up to 10. For $R=2 / 3$, the girth is 4 , and therefore, the method is able to find the number of short cycles of lengths 4 and 6 . For each of the four codes, the proposed algorithm takes less than one second to find the results of Table 4.2.

An inspection of the results in Table 4.2 demonstrates that, in general, the number of short cycles increases with the increase in rate. This is a consequence of the Tanner 
graph becoming denser. In fact the density of the graph, defined as the ratio of the number of edges to the product $n \times m$, is equal to 0.299, 0.458, 0.590, and 0.822, for the four rates, respectively.

Table 4.2: Number of Short Cycles in the Tanner Graphs of LDPC Codes of Block Length $n=1944$ with Different Rates Adopted in IEEE 802.11 Standard [41]

\begin{tabular}{|c|c|c|c|c|}
\hline \multirow{2}{*}{$\begin{array}{c}\text { Cycle } \\
\text { length }\end{array}$} & \multicolumn{4}{|c|}{$n=1944$} \\
\cline { 2 - 5 } & $R=1 / 2$ & $R=2 / 3$ & $R=3 / 4$ & $R=5 / 6$ \\
\hline \hline 4 & 0 & 81 & 0 & 0 \\
\hline 6 & 3321 & 6399 & 9558 & 14418 \\
\hline 8 & 70551 & - & 346923 & 563193 \\
\hline 10 & 1465371 & - & 11269044 & 19125801 \\
\hline
\end{tabular}

For the block lengths 1296 and 648, the codes are constructed based on base graphs with sizes $\left(\left|W_{b}\right|,\left|U_{b}\right|\right)$ similar to those of $n=1944$ (but with different degree distributions) and by using liftings of degree $N=54$ and 27, respectively. Tables 4.3 and 4.4 show the number of short cycles obtained by the proposed method for the two block lengths, respectively. Trends similar to those of Table 4.2 are also observed here. For each code in Table 4.3, the proposed algorithm takes less than 0.5 seconds to find the results. The time for each code of Table 4.4 is less than 0.2 seconds.

Table 4.3: Number of Short Cycles in the Tanner Graphs of LDPC Codes of Block Length $n=1296$ with Different Rates Adopted in IEEE 802.11 Standard [41]

\begin{tabular}{|c|c|c|c|c|}
\hline \multirow{2}{*}{$\begin{array}{c}\text { Cycle } \\
\text { length }\end{array}$} & \multicolumn{4}{|c|}{$n=1296$} \\
\cline { 2 - 5 } & $R=1 / 2$ & $R=2 / 3$ & $R=3 / 4$ & $R=5 / 6$ \\
\hline \hline 4 & 0 & 108 & 0 & 0 \\
\hline 6 & 2754 & 7830 & 14040 & 22842 \\
\hline 8 & 68229 & - & 512433 & 1141830 \\
\hline 10 & 1470906 & - & 17523054 & 46268496 \\
\hline
\end{tabular}

Example 5. In this example, we use the proposed algorithm to find the girth distribution of QC protograph $L D P C$ codes and compare it with that of random LDPC 
Table 4.4: Number of Short Cycles in the Tanner Graphs of LDPC Codes of Block Length $n=648$ with Different Rates Adopted in IEEE 802.11 Standard [41]

\begin{tabular}{|c|c|c|c|c|}
\hline \multirow{2}{*}{$\begin{array}{c}\text { Cycle } \\
\text { length }\end{array}$} & \multicolumn{4}{|c|}{$n=648$} \\
\cline { 2 - 5 } & $R=1 / 2$ & $R=2 / 3$ & $R=3 / 4$ & $R=5 / 6$ \\
\hline \hline 4 & 0 & 0 & 54 & 0 \\
\hline 6 & 3942 & 8046 & 13662 & 32346 \\
\hline 8 & 123012 & 193023 & - & 1574775 \\
\hline 10 & 2760507 & 5288679 & - & 68153562 \\
\hline
\end{tabular}

codes. We generate 10,000 samples for each length of each category of codes. In the $Q C$ protograph construction, we use a fully-connected base graph of size $3 \times 6$ and select the permutations uniformly at random from $\{0,1, \ldots, N-1\}$, where $N$ is the lifting degree. In the random constructions, Tanner graphs with variable and check degrees 3 and 6 are constructed randomly with the constraint that the girth of each graph must be larger than 4. This resulted in Tanner graphs that almost always had a girth of 6 . For the QC protograph codes, the (average) girth distributions are given in Table 4.5 for different lifting degrees. The table shows that as the length (lifting degree) increases, the girth distribution improves, and is in general significantly superior to the girth distribution of similar random codes (which almost always have $g=6$, regardless of the block length). For example, for $N=10,000$, corresponding to the code block length of 60,000, all the randomly generated $Q C$ codes have girth larger than 6 with only $5.8 \%$ of them having $g=8$. It should be noted that, for the base graph used in this example, the girth of the protograph codes can not be larger than 12 [32]. We have also studied the girth distributions of QC protograph codes for different rates and different base graphs. The results show generally similar trends as those of Table 4.5, and are thus omitted. 
Table 4.5: Girth Distribution of QC LDPC Codes with a $3 \times 6$ Fully Connected Base Graph and Different Lifting Degrees

\begin{tabular}{|c|c|c|c|c|c|}
\hline \multirow{2}{*}{$\begin{array}{c}\text { Lifting } \\
\text { Degree }\end{array}$} & \multicolumn{5}{|c|}{ Girth } \\
\cline { 2 - 6 } & $g=4$ & $g=6$ & $g=8$ & $g=10$ & $g=12$ \\
\hline \hline $\mathbf{1 0 0}$ & $37.5 \%$ & $45.2 \%$ & $17.3 \%$ & 0 & 0 \\
\hline $\mathbf{5 0 0}$ & $8.5 \%$ & $19.9 \%$ & $67.8 \%$ & $3.8 \%$ & 0 \\
\hline $\mathbf{1 0 0 0}$ & $4.4 \%$ & $10.8 \%$ & $63.9 \%$ & $20.8 \%$ & $0.1 \%$ \\
\hline $\mathbf{2 0 0 0}$ & $2.1 \%$ & $6.5 \%$ & $42.2 \%$ & $44.7 \%$ & $4.5 \%$ \\
\hline $\mathbf{4 0 0 0}$ & 0 & $1.5 \%$ & $27.1 \%$ & $47.7 \%$ & $23.7 \%$ \\
\hline $\mathbf{1 0 0 0 0}$ & 0 & 0 & $5.8 \%$ & $27.5 \%$ & $66.7 \%$ \\
\hline
\end{tabular}




\section{Chapter 5}

\section{On the Girth of Quasi Cyclic Protograph LDPC Codes}

In this chapter, we study the relationships between the short cycles of a lifted graph, the size of the base graph and the lifting degree. For this, we closely examine the relationships among the subgraphs of the base graph that give rise to short cycles in the lifted graph. As a result of this study, we derive a lower bound on the required lifting degree for having a specific girth in the protograph construction using a given base graph, or equivalently, an upper bound on the girth of a lifted graph constructed using a given base graph and a given lifting degree.

\subsection{Images of Cycles of Cyclic Liftings in the Base Graph}

It is known that the structures which map to cycles in a lifted graph are tailless backtrackless closed walks in the base graph [34]. Consider a walk $W$ of length $l$ in $G$, with the sequence of edges $e_{1}, \ldots, e_{l}$. Suppose that, associated with the edges, we have the sequence of permutation shifts $p_{1}, \ldots, p_{l}$, corresponding to a cyclic lifting of degree $N$. The permutation shift of $W$ is defined as [34]:1

$$
p_{W}=\sum_{i=0}^{l-1}(-1)^{i} p_{i+1} \bmod N
$$

In the rest of the chapter, for simplicity of presentation, we sometimes use the

\footnotetext{
${ }^{1}$ For more recent references, the reader is referred to [2] and [3].
} 
notation $(\cdot)_{N}$ to denote modulo $N$ operation. We also sometimes drop the notation altogether when there is no possibility of confusion.

The following theorem gives necessary and sufficient conditions for a tailless backtrackless closed walk to map to cycle(s) in the lifted graph.

Theorem 3 ( [34]). ${ }^{1}$ Consider a cyclic $N$-lifting $\tilde{G}$ of $G$, and let $W$ be a tailless backtrackless closed walk of length $l$ in $G$. Then the inverse image of $W$ is a set of $l$-cycles in the lifted graph $\tilde{G}$ if and only if $p_{W}$ is 0 and $W$ does not contain any tailless backtrackless closed walk of length shorter than $l$ with the permutation shift of 0 .

According to Theorem 3, the permutation shift $p_{W}$ of a walk $W$ in the base graph, and consequently the permutation shift assignment to the edges of the walk, play a crucial role in whether the walk is mapped to a cycle or not. However, there are situations where the permutation shift of a walk is zero, independent of the permutations assigned to the edges of the walk. This can happen when the number of traversals of each edge in one direction is the same as that in the opposite direction. In this case, the permutation shift of the walk is always zero. The structures with this property are called inevitable cycles [56]. Figures 5.1(a) and 5.1(b) show two important classes of inevitable cycles, called the theta graph and the dumbbell graph, respectively [34].

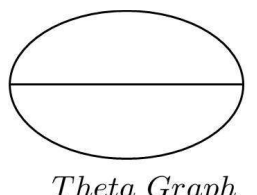

(a)

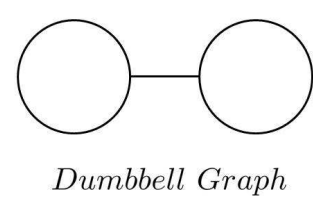

(b)

Figure 5.1: Inevitable cycles: a) Theta graph, b) Dumbbell Graph.

By finding the inevitable cycles of the base graph, one can provide an upper bound on the girth of the lifted graph. This is the idea used in [56], [57], [59] and [80]. In this work, we use the dependencies among the permutation shifts of different backtrackless tailless closed walks of the base graph. We show that depending on the base graph and the lifting degree and regardless of the values of permutation shifts, the permutation shift of some of the tailless backtrackless closed walks shorter than the inevitable cycles may necessarily be zero. This then dictates an upper bound on the girth of the lifted graph, which is tighter than the one imposed by inevitable cycles. 


\subsection{Derivation of Bounds}

To derive the bounds in this section, we consider necessary conditions required for a lifted graph to have a certain girth $g$. For this, we examine certain tailless backtrackless closed walks $W$ of the base graph whose lengths are less than $g$ and require that their permutation shifts $p_{W}$ be nonzero. In general, however, we do not necessarily consider all such walks and thus the necessary conditions are in general not sufficient.

In this work, we consider base graphs with no parallel edges. This implies that the girth of the base graph is at least 4 . Considering Lemma 2, it thus turns out that all the tailless backtrackless closed walks of length 4 and 6 in such base graphs are cycles.

\subsubsection{Necessary Conditions for $g \geq 6$}

According to the above discussion, all the tailless backtrackless closed walks of length 4, which may be mapped to 4-cycles of the lifted graph, are 4-cycles in the base graph. To avoid 4-cycles in the lifted graph, one thus needs to assign the edge permutation shifts such that the permutation shifts of all the 4-cycles of the base graph will be non-zero. In the following, we study the dependencies between the permutation shifts of 4-cycles which have exactly 2 edges in common.

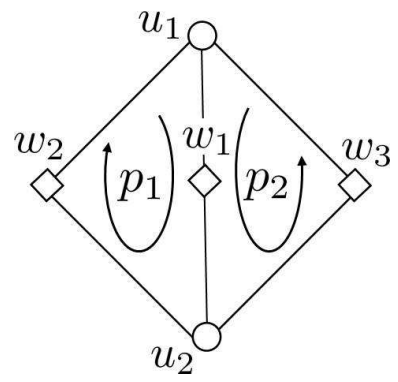

Figure 5.2: Dependencies between permutation shifts of 4-cycles.

Figure 5.2 shows three 4-cycles $C_{1}: u_{1}, w_{1}, u_{2}, w_{2}, u_{1}, C_{2}: u_{1}, w_{1}, u_{2}, w_{3}, u_{1}$, and $C_{3}: u_{1}, w_{3}, u_{2}, w_{2}, u_{1}$. Let $p_{1}$ and $p_{2}$ be the permutation shifts of cycles $C_{1}$ and $C_{2}$, respectively. It is then easy to check that the permutation shift of $C_{3}$ is $p_{3}=p_{1}-p_{2}$. This means that if $p_{1}=p_{2}$, then $p_{3}$ will be zero, and therefore $C_{3}$ will be mapped to a set of $N$ 4-cycles in the lifted graph. This implies that to have a lifted graph with a 
girth greater than 4 , all the cycles of length 4 in the base graph which share exactly 2 edges must have different permutation shifts.

Consider a base graph and all the distinct 4-cycles in it that share only two variable nodes $u_{i}$ and $u_{j}$ and one check node $w$ that is connected to both variable nodes. Since there are no parallel edges in the base graph, all such cycles also share the two edges $\left(u_{i}, w\right)$ and $\left(u_{j}, w\right)$. (See, e.g., Figure 5.2.) Suppose that the columns of the base matrix corresponding to $u_{i}$ and $u_{j}$ are denoted by $c_{i}$ and $c_{j}$, respectively. In the absence of parallel edges in the base graph, it is easy to see that, for any check node $w$ connected to both $u_{i}$ and $u_{j}$, the number of 4-cycles described above is $\alpha\left(c_{i}, c_{j}\right)-1$, where $\alpha\left(c_{i}, c_{j}\right)$ is the number of positions in which both $c_{i}$ and $c_{j}$ have nonzero values. Similarly, $\alpha\left(r_{i}, r_{j}\right)-1$ is the number of distinct 4-cycles that only share the two check nodes corresponding to rows $r_{i}$ and $r_{j}$ of the base matrix and a variable node that is connected to both check nodes. Let $\alpha_{r, \max }=\max \left\{\alpha\left(r_{i}, r_{j}\right)-1: 1 \leq i, j \leq m, i \neq j\right\}$, and $\alpha_{c, \max }=\max \left\{\alpha\left(c_{i}, c_{j}\right)-1: 1 \leq i, j \leq n, i \neq j\right\}$. Then the lifting degree $N$ must be at least $\alpha_{\max }=\max \left\{\alpha_{r, \max }, \alpha_{c, \max }\right\}+1$ to allow all the 4-cycles sharing only two edges to have different non-zero permutation shifts.

Lemma 5. For any $Q C$ protograph code constructed using a base graph with no parallel edges, a necessary condition for having a girth of at least 6 is to have the lifting degree $N \geq \alpha_{\max }$, where $\alpha_{\max }$ is the maximum number of common non-zero positions between any two rows or any two columns of the base matrix.

It should be mentioned that since the size of the base graphs is usually small, it is computationally very easy to find $\alpha_{\max }$.

For the case where the base graph is an $m \times n$ fully-connected graph, $\alpha\left(r_{i}, r_{j}\right)=n$, for all $i \neq j$, and $\alpha\left(c_{i}, c_{j}\right)=m$, for all $i \neq j$. Therefore, $N$ must be chosen to be at least $\alpha_{\max }=\max \{m, n\}=n$.

Corollary 1 ( [32], Corollary 2.2). In constructing a cyclically lifted graph with lifting degree $N$ using an $m \times n$ fully-connected base graph with no parallel edges, a necessary condition for having a girth of at least 6 for the lifted graph is $N \geq n$. 
Example 6. Consider the following matrix of permutation shifts:

$$
P=\left[\begin{array}{ccccc}
0 & 0 & 0 & \ldots & 0 \\
0 & 1 & 2 & \ldots & n-1 \\
0 & 2 & 4 & \ldots & (2 n-4)_{N} \\
\vdots & \vdots & \vdots & \ddots & \vdots \\
0 & m-1 & (2 m-2)_{N} & \ldots & ((m-1)(n-1))_{N}
\end{array}\right]
$$

where $(k)_{N}$ denotes the value of $k$ modulo $N$. If the fully-connected base graph is lifted based on $P$ and with the degree of lifting $N$, where $N$ is the smallest prime number satisfying $N \geq n$, then the lifted graph has a girth of $6 .^{2}$ In the case that $n$ itself is a prime number (and thus $N=n$ ), the bound of Corollary 1 is tight and the lifted codes are called array based LDPC codes [30]. Based on Corollary 1, for a prime n, array based LDPC codes are the smallest possible QC protograph codes with girth 6 resulting from a fully-connected base graph.

\subsubsection{Necessary Conditions for $g \geq 8$}

For a base graph with no parallel edges, all the tailless backtrackless closed walks of length 6 are cycles. That is, the only structure which may be mapped to a 6-cycle in the lifted graph is also a 6-cycle in the base graph. To avoid 6-cycles in the lifted graph, one thus needs to assign the edge permutation shifts such that the permutation shifts of all 6-cycles of the base graph will be non-zero.

Consider the structure of Figure 5.3, where two 4-cycles share an edge $e=\left(u_{1}, w_{1}\right)$. In Figure 5.3, there are two 4-cycles $C_{1}: u_{1}, w_{1}, u_{3}, w_{3}, u_{1}, C_{2}: u_{1}, w_{1}, u_{2}, w_{2}, u_{1}$, and a 6-cycle $C_{3}: u_{1}, w_{2}, u_{2}, w_{1}, u_{3}, w_{3}, u_{1}$. Let $p_{1}$ and $p_{2}$ be the permutation shifts of cycles $C_{1}$ and $C_{2}$, respectively. It is then easy to check that the permutation shift of

\footnotetext{
${ }^{2}$ Consider the 4-cycle between variable nodes $i_{1}$ and $i_{2}$ and check nodes $j_{1}$ and $j_{2}$, where $1 \leq$ $i_{1}, i_{2} \leq n$ and $1 \leq j_{1}, j_{2} \leq m$, respectively. The permutation shift of this cycle is $p=\left(i_{2}-i_{1}\right)\left(j_{1}-j_{2}\right)$ $\bmod N$. As $i_{2} \neq i_{1}, j_{1} \neq j_{2}$ and $N$ is prime, $p$ will be non-zero. That is the permutation shift of any 4 -cycle in the base graph is non-zero, and thus, the girth of the lifted graph is at least 6 . Moreover, if $m, n \geq 3$, the permutation shift of the 6 -cycle $w_{2}, u_{1}, w_{3}, u_{2}, w_{1}, u_{3}, w_{2}$ is $p_{21}-p_{31}+p_{32}-p_{12}+$ $p_{13}-p_{23}=0-0+2-0+0-2=0$, where $u_{i}$ and $w_{j}$ denote the variable node and the check node corresponding to the $i^{\text {th }}$ column and the $j^{\text {th }}$ row of the base matrix, respectively. The girth of the resultant graph is thus 6 .
} 


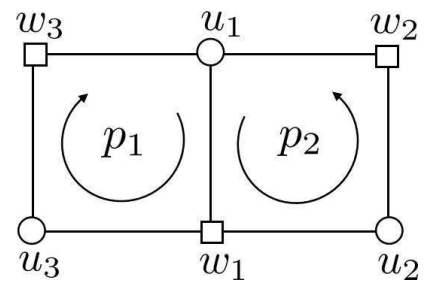

Figure 5.3: Dependencies between the permutation shifts of two 4-cycles having one edge in common.

$C_{3}$ is $p_{3}=p_{1}-p_{2}$. This means that if $p_{1}=p_{2}$, then $p_{3}$ will be zero, and therefore $C_{3}$ will be mapped to a set of 6 -cycles in the resultant lifted graph. This implies that to avoid cycles of length 6 in the lifted graph, all the cycles of length 4 in the base graph which share exactly one edge must have different permutation shifts. We are thus interested in counting such cycles.

\section{B.1. Fully-connected base graphs}

First, we consider the special case where the base matrix is an all-one matrix (fullyconnected base graph). For a given edge $e=\left(u_{i_{1}}, w_{j_{1}}\right)$, we consider all the 4-cycles containing $e$. All such cycles can be represented in the form of $u_{i_{1}}, w_{j_{1}}, u_{i_{2}}, w_{j_{2}}, u_{i_{1}}$, with different values for $i_{2}$ and $j_{2}$. For an $m \times n$ fully-connected base graph, $i_{2}$ can be any value between 1 and $n$, except $i_{1}$ (i.e., $1 \leq i_{2} \leq n$ and $i_{2} \neq i_{1}$ ). This means that there are $n-1$ choices for $i_{2}$. Similarly, the choices for $j_{2}$ are $1 \leq j_{2} \leq m$ and $j_{2} \neq j_{1}$, i.e., there are $m-1$ possible choices for $j_{2}$. This means that there are $(n-1)(m-1)$ distinct 4-cycles which contain $e$. These cycles can share only one edge (edge $e$ ) or two edges (edge $e$ and another edge), but not three edges because there are no parallel edges in the graph. For the lifted graph to have a girth of at least 8, all such 4-cycles must have different non-zero permutation shifts. Satisfying this condition will also satisfy the necessary condition discussed in Section III.A for avoiding 4-cycles in the lifted graph.

Lemma 6. For any QC protograph code constructed using an $m \times n$ fully-connected base graph with no parallel edges, a necessary condition for having a girth of at least 8 is that the lifting degree $N$ satisfies $N \geq(n-1)(m-1)+1$.

Example 7. According to Lemma 6, in order to have a girth of at least 8 in a protograph construction using a fully-connected $3 \times 5$ base graph, the lifting degree must be $N \geq 9$. The following permutation shift matrix with a lifting degree $N=13$, 
found by a heuristic search, results in a lifted graph with girth 8.

$$
P=\left[\begin{array}{lllll}
1 & 7 & 0 & 10 & 7 \\
3 & 6 & 5 & 1 & 7 \\
6 & 8 & 9 & 9 & 5
\end{array}\right]
$$

In Section $V$, we present new systematic constructions of cyclic liftings with girth 8 based on fully-connected base graphs with variable node degrees 3, 4, 5 and 6 .

B.2. Arbitrary base graphs

For an arbitrary regular or irregular base graph, the minimum lifting degree for having a girth of at least 8, can similarly be determined based on the maximum number of 4-cycles sharing a specific edge.

Lemma 7. For any QC protograph code constructed using a base graph with no parallel edges, a necessary condition for having a girth of at least 8 is that the lifting degree $N$ satisfies $N>\max \left\{N_{4}^{e_{i}}\right\}$, where the maximum is taken over all the edges of the base graph, i.e., $1 \leq i \leq|E|$, and $N_{4}^{e_{i}}$ is the number of distinct 4-cycles containing the edge $e_{i}$.

It should be mentioned that $N_{4}^{e_{i}}$ can be computed efficiently by subtracting the number of distinct 4-cycles of the base graph after removing $e_{i}$ from the graph, from the total number of distinct 4-cycles in the graph. To count the number of distinct 4cycles of the base graph in both cases (with an without $e_{i}$ ), one can use, for example, Algorithm 1. Moreover, it is easy to check that for any edge $e_{i}=\{u, w\}, N_{4}^{e_{i}} \leq$ $\left(d_{u}-1\right)\left(d_{w}-1\right)$. This upper bound can be used to find an approximation for the lower bound on $N$.

Example 8. Consider the irregular base graph shown in Figure 5.4. This graph consists of three variable nodes of degree 4, two variable nodes of degree 3 and two variable nodes of degree 2. There are four check nodes with degrees 4, 5, 6 and 7 . According to Lemma 7, in order to have a girth of at least 8 in the QC protograph construction using this base graph, the lifting degree must be at least 13 . This is obtained based on the number of 4-cycles containing the edge connecting $u_{1}$ and $w_{3}$ in Figure 5.4, which is 12. By a heuristic search, we were able to find the following permutation shift matrix: 


$$
P=\left[\begin{array}{ccccccc}
3 & 6 & 10 & 1 & +\infty & 13 & 10 \\
4 & 0 & 2 & 0 & 0 & +\infty & +\infty \\
9 & 0 & 8 & 3 & 11 & 6 & 6 \\
9 & 1 & 0 & +\infty & 2 & +\infty & +\infty
\end{array}\right],
$$

which combined with a lifting of degree $N=15$ results in a lifted graph with girth 8 .

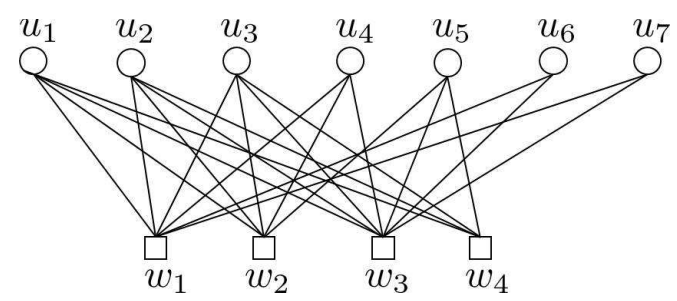

Figure 5.4: Irregular base graph of Example 8.

\subsubsection{Necessary Conditions for $g \geq 10$}

Figure 5.5 shows all the possible tailless backtrackless closed walks of length 8 in a base graph with no parallel edges. Figure 5.5(a) is a simple cycle of length 8 . In Figures 5.5(b), 5.5(c) and 5.5(d), the walks consist of two 4-cycles which share one node, two nodes (one edge) and three nodes (two edges), respectively. One of the possible walks for the case of Figure 5.5(c) is specified using the arrows in the figure. In Figure 5.5(e), the walks go through the 4-cycle twice.

We first consider the case of Figure 5.5(b). Starting from node $w_{1}$, one possible tailless backtrackless closed walk is $W_{1}: w_{1}, u_{1}, w_{2}, u_{2}, w_{1}, u_{3}, w_{3}, u_{4}, w_{1}$, and another one is $W_{2}: w_{1}, u_{1}, w_{2}, u_{2}, w_{1}, u_{4}, w_{3}, u_{3}, w_{1}$. Let the permutation shifts of the 4-cycles $w_{1}, u_{1}, w_{2}, u_{2}, w_{1}$ and $w_{1}, u_{4}, w_{3}, u_{3}, w_{1}$ be $p_{1}$ and $p_{2}$, respectively (see Figure 5.5(b)). The permutation shift $p_{W_{1}}$ of walk $W_{1}$ is then $p_{W_{1}}=p_{1}-p_{2}$, and the permutation shift $p_{W_{2}}$ of walk $W_{2}$ is $p_{W_{2}}=p_{1}+p_{2}$. In order to have a girth greater than 8 , the permutation shift of such walks must be non-zero. That is, the permutation assignment of the edges must be such that $p_{1} \neq p_{2}$ and $p_{1} \neq\left(-p_{2}\right)_{N}$. In other words, the permutation shifts of all the 4-cycles sharing one specific node must be different from each other and be different from the additive inverse of each other. (Note that 


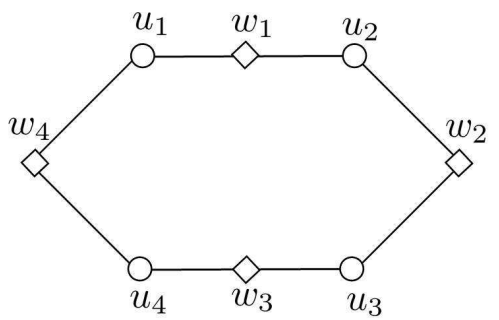

(a)

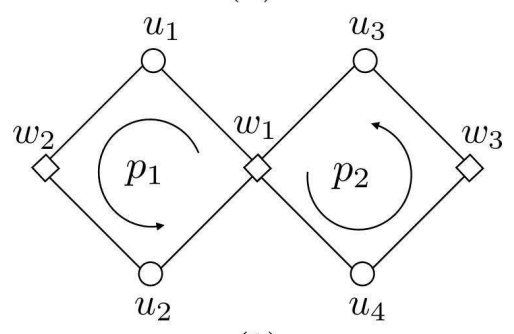

(b)

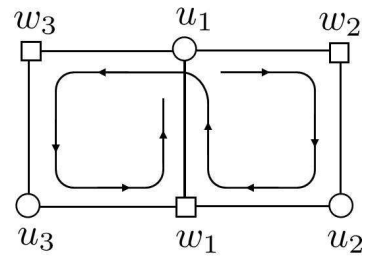

(c)

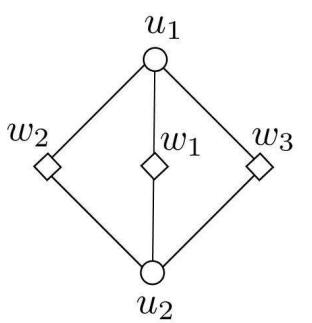

$(d)$

Figure 5.5: Structures in a base graph which may be mapped to 8-cycles in the lifted graph.

in addition to $W_{1}$ and $W_{2}$, there are many other tailless backtrackless closed walks of length 8 in Figure 5.5(b). However, it is easy to check that the permutation shift of each of these walks is one of the values $p_{1}+p_{2}, p_{1}-p_{2},-p_{1}-p_{2}$, or $-p_{1}+p_{2}$. It can thus be seen that such walks will not provide any additional constraints on the permutation shifts $p_{1}$ and $p_{2}$.)

A similar investigation of Figures 5.5(c) and 5.5(d) reveals that to avoid the 8cycles in the lifted graph caused by the tailless backtrackless closed walks in these figures, the permutation shifts of all the 4-cycles that share two nodes (one edge) or three nodes (two edges) must be different from each other and be different from the additive inverse of each other. Satisfying these conditions will also satisfy the necessary conditions discussed in Sections III.A and III.B, for avoiding 4-cycles and 6 -cycles in the lifted graph, respectively.

For the case of Figure 5.5(e), one possible tailless backtrackless closed walk of length 8 is $W_{3}: w_{1}, u_{1}, w_{2}, u_{2}, w_{1}, u_{1}, w_{2}, u_{2}, w_{1}$. Let the permutation shift of the 4-cycle $w_{1}, u_{1}, w_{2}, u_{2}, w_{1}$ be $p_{1}$. Therefore the permutation shift $p_{W_{3}}$ of walk $W_{3}$ is 
$p_{W_{3}}=p_{1}+p_{1}=\left(2 p_{1}\right)_{N}$. That is, for having a non-zero $p_{W_{3}},\left(2 p_{1}\right)_{N} \neq 0$, or equivalently $p_{1} \neq 0$ and $p_{1} \neq N / 2$ for an even $N$, and $p_{1} \neq 0$ for an odd $N$.

Given a base graph, by enforcing all (or some of) the constraints discussed above on the permutation shifts of 4-cycles involved in structures of Figures 5.5(b)-(e), one can derive a lower bound on the lifting degree that would result in a lifted graph with girth at least 10. In the following, we provide such bounds starting with the special case of a fully-connected base graph.

\section{C.1. Fully-connected base graphs}

Consider the structure of Figure 5.5(b). There are several ways to count the number of distinct 4-cycles sharing a specific node. For instance, one approach is to subtract the number of 4-cycles of the base graph after removing the node from the graph, from the total number of 4-cycles in the graph. For an $m \times n$ fully-connected base graph, the number of distinct 4-cycles is $\left(\begin{array}{c}n \\ 2\end{array}\right)\left(\begin{array}{c}m \\ 2\end{array}\right)=\frac{n m(n-1)(m-1)}{4}$. Removing any variable node, the number of 4-cycles in the resulting graph is $\frac{(n-1) m(n-2)(m-1)}{4}$. Similarly, after removing a check node, the number of 4-cycles in the new graph is $\frac{n(m-1)(n-1)(m-2)}{4}$. This implies that for a fully-connected base graph, each variable node is contained in $m(m-1)(n-1) / 2$-cycles and each check node is contained in $n(n-1)(m-1) / 24$-cycles. The consequence of this result and the discussion presented earlier on the tailless backtrackless closed walks of Figures 5.5(b)-(e) is the following lemma.

Lemma 8. For any QC protograph code constructed using an $m \times n$ fully-connected base graph, a necessary condition to have a girth of at least 10 is that the lifting degree $N$ satisfies $N \geq n(n-1)(m-1)+1$.

The fact that any two distinct 4-cycles which have at least one node in common must have non-zero permutation shifts different from each other and different from the additive inverse of each other can be used to tighten the bound for special cases. For instance, for a $3 \times n$ fully-connected base graph, it is easy to check that any two 4-cycles in the base graph have at least one check node in common. This together with the fact that there are $3 n(n-1)$ distinct 4-cycles in such a base graph results in the following lemma.

Lemma 9. For any $Q C$ protograph code constructed using a $3 \times n$ fully-connected base graph, a necessary condition to have a girth of at least 10 is that the lifting degree $N$ satisfies $N \geq 3 n(n-1)+1$. 
Example 9. Consider the TSF $(155,64)$ code [100], constructed using a fullyconnected base graph of size $3 \times 5$ and a lifting degree of 31 . The maximum girth for the Tanner graph of this code based on inevitable cycles is 12. A tighter upper bound of 10 on the girth of this construction was derived in [101] using the wellknown tree bound [33]. Based on Lemma 9, however, the minimum lifting degree using this base graph which can result in a girth larger than 8 for the lifted graph is $3 n(n-1)+1=61$. That is, using this base graph and the lifting degree 31 , the girth of all possible lifted graphs is at most 8, regardless of the edge permutations used. It should be mentioned that the girth of TSF $(155,64)$ code is also 8, which is the highest possible based on Lemma 9. A construction which achieves the bound of 61, i.e., a lifting of degree 61 and girth 10, is also presented in [100].

\section{C.2. Arbitrary base graphs}

For an arbitrary regular or irregular base graph, we have the following result.

Lemma 10. For any QC protograph code constructed using a base graph with no parallel edges, a necessary condition to have a girth of at least 10 is that the lifting degree $N$ satisfies $N>2 \times N_{4, \max }$, where $N_{4, \max }$ is the maximum number of distinct 4-cycles sharing one node of the base graph.

Note that $N_{4, \max }=\max \left\{N_{4}^{u_{i}}, N_{4}^{w_{j}}\right\}$, where $N_{4}^{u_{i}}$ and $N_{4}^{w_{j}}$ are the numbers of distinct 4 -cycles containing the variable node $u_{i}$ and the check node $w_{j}$, respectively, and the maximum is taken over all variable nodes $u_{i}, 1 \leq i \leq n$, and all check nodes $w_{j}, 1 \leq j \leq m$, of the base graph.

Note that, as the size of base graphs is usually small, it is easy to find the values $N_{4}^{u_{i}}$ and $N_{4}^{w_{j}}$. It is also straightforward to see that for any variable node $u_{i}, N_{4}^{u_{i}} \leq$ $\left(\begin{array}{c}d_{u_{i}} \\ 2\end{array}\right)\left(d_{w, \text { max }}-1\right)$, and for any check node $w_{j}, N_{4}^{w_{j}} \leq\left(\begin{array}{c}d_{w_{j}} \\ 2\end{array}\right)\left(d_{u, \text { max }}-1\right)$, where $d_{w, \text { max }}$ and $d_{u, \max }$ are the maximum check node degree and the maximum variable node degree in the base graph, respectively. These upper bounds can be used to obtain an approximation for the lower bound on $N$.

Remark 1. One should note that, in addition to the constraints used in this work to derive the bounds, there are other constraints that need to be satisfied to achieve a certain girth. For example, suppose that one is interested in having $g>8$, and consider the graphical structure presented in Figure 5.5(d). For convenience, this structure is also shown in Figure 5.6. 


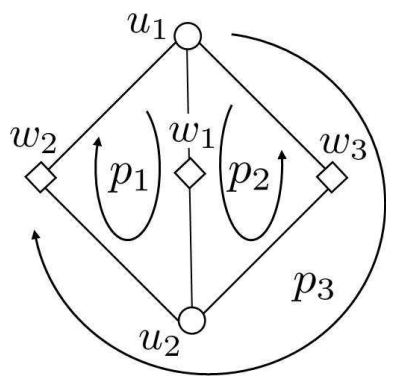

Figure 5.6: Dependencies between permutation shifts of 4-cycles.

In this figure, consider the three 4-cycles $C_{1}: u_{1}, w_{1}, u_{2}, w_{2}, u_{1} ; \quad C_{2}$ : $u_{1}, w_{1}, u_{2}, w_{3}, u_{1}$; and $C_{3}: u_{1}, w_{3}, u_{2}, w_{2}, u_{1}$. Let $p_{1}, p_{2}$ and $p_{3}$ be the permutation shifts of cycles $C_{1}, C_{2}$ and $C_{3}$, respectively. By careful examination of Figure 5.6, and by considering that $p_{3}=p_{1}-p_{2}$, one can show that the following constraints are necessary to ensure that the girth of the lifted graph is larger than 8:

$$
\left\{\begin{array}{l}
p_{1} \neq 0 \\
p_{2} \neq 0 \\
p_{1} \neq \pm p_{2} \\
2 p_{1} \neq p_{2}, \\
p_{1} \neq 2 p_{2} .
\end{array}\right.
$$

For instance, the last inequality comes from the fact that the walk $u_{1}, w_{3}, u_{2}, w_{2}, u_{1}, w_{3}, u_{2}, w_{1}, u_{1}$ is a tailless backtrackless closed walk of length 8 with permutation shift $p_{3}-p_{2}=p_{1}-2 p_{2}$.

Although we have not been able to use the additional constraints such as those explained in Remark 1 to tighten the bounds, one can still use them to devise the permutation shift matrix for achieving a certain girth.

\subsection{New Constructions of QC LDPC Codes with Girth 8}

In this section, we present new constructions with girth 8 for QC LDPC codes using fully-connected base graphs with variable node degrees $3,4,5$ and 6 . The corresponding QC LDPC codes are thus regular with rates $1-3 / n, 1-4 / n, 1-5 / n$ and 
$1-6 / n$, respectively. These constructions provide upper bounds on the lifting degrees required for a given base graph to achieve girth 8 for the lifted graph.

Lemma 11. Suppose that the following permutation shift matrix $P$ is used to construct a cyclic $N$-lifting $\tilde{G}$ of a fully-connected $3 \times n$ base graph $G$ with $N>n(n-1)$ :

$$
P=\left[\begin{array}{ccccc}
0 & 0 & 0 & \ldots & 0 \\
0 & 1 & 2 & \ldots & n-1 \\
0 & n & 2 n & \ldots & (n-1) n
\end{array}\right]
$$

Then, $\tilde{G}$ has girth 8 .

Proof. It is easy to check that the girth of the lifted graph is not 4 . If there exists a cycle of length 6 in the lifted graph, its image in the base graph must be a 6 -cycle with zero permutation shift. Now, consider a 6 -cycle $C$ in the base graph. Since the base graph has only 3 check nodes, any 6-cycle must contain all the check nodes. Let the three check nodes be labeled by 1,2 and 3, corresponding to the three rows of $P$, respectively, and consider the labeling of the variable nodes in the base graph from 1 to $n$, corresponding to the columns of $P$, respectively. Let $i_{1}$ and $i_{2}$ in $[1, n], i_{1} \neq i_{2}$, correspond to the variable nodes in $C$ that are connected to the first check node. Cycle $C$ can then be represented by one of the following two sequences of edges $e_{1, i_{1}}, e_{1, i_{2}}, e_{2, i_{2}}, e_{2, i_{3}}, e_{3, i_{3}}, e_{3, i_{1}}$, or $e_{1, i_{1}}, e_{1, i_{2}}, e_{3, i_{2}}, e_{3, i_{3}}, e_{2, i_{3}}, e_{2, i_{1}}$, where $e_{j, i}$ is the edge connecting the $j^{\text {th }}$ check node to the $i^{\text {th }}$ variable node, and $1 \leq i_{1}, i_{2}, i_{3} \leq n$ and $i_{1} \neq i_{2} \neq i_{3}$. The permutation shifts corresponding to the two sequences are:

$$
\begin{aligned}
& p_{1}=p_{1 i_{1}}-p_{1 i_{2}}+p_{2 i_{2}}-p_{2 i_{3}}+p_{3 i_{3}}-p_{3 i_{1}} \\
& =0-0+\left(i_{2}-i_{3}\right)+n\left(i_{3}-i_{1}\right) \quad \bmod N
\end{aligned}
$$

and

$$
\begin{aligned}
& p_{2}=p_{1 i_{1}}-p_{1 i_{2}}+p_{3 i_{2}}-p_{3 i_{3}}+p_{2 i_{3}}-p_{2 i_{1}} \\
& =0-0+n\left(i_{2}-i_{3}\right)+\left(i_{3}-i_{1}\right) \bmod N
\end{aligned}
$$

respectively, where $p_{j i}$ denotes the element of $P$ in row $j$ and column $i$. Considering that $N>n(n-1)$ and that $i_{1}, i_{2}$ and $i_{3}$ are distinct integer numbers between 1 and $n$, it is easy to check that neither $p_{1}$ nor $p_{2}$ can be zero. This proves that any 
6-cycle in the base graph has a non-zero permutation shift. It is also easy to find a tailless backtrackless closed walk of length 8 with zero permutation shift in the base graph. One example is $w_{1}, u_{1}, w_{2}, u_{2}, w_{1}, u_{3}, w_{2}, u_{2}, w_{1}$ which has a permutation shift of $p_{11}-p_{21}+p_{22}-p_{12}+p_{13}-p_{23}+p_{22}-p_{12}=0-0+1-0+0-2+1-0=0$, where $u_{i}$ and $w_{j}$ denote the $i$ th variable node and the $j$ th check node in the base graph, respectively. The girth of the resultant graph is thus 8 .

It should be mentioned that for array based LDPC codes with left degree 3, it is known that the minimum distance is 6 regardless of the code length [125]. It can be shown however that the construction of Lemma 11, results in codes with a minimum distance at least 8 (see Appendix A, Lemma 20). Moreover, applying a permutation to the second row of the permutation shift matrix of Lemma 11 with a lifting degree $N$ larger than $(2 n-1)(n-1)$ results in a minimum distance of at least 10 (see Appendix B, Lemma 21).

Another advantage of the proposed construction compared to array based LDPC codes is the higher flexibility in selecting the block length of the lifted code. While the block length for the proposed construction is $N \times n$ and can thus be changed by selecting any value of $N$ larger than $n(n-1)$, the block length for the array based LDPC codes can only be $q^{2}$, where $q=n$ is a prime number. It should however be noted that for the proposed construction, the minimum lifting degree increases with $n^{2}$, and thus the block length of the lifted code increases by at least $n^{3}$. This implies that the proposed construction may be more suitable for rather small base graphs.

Lemma 12. Suppose that the following permutation shift matrix $P$ is used to construct a cyclic $N$-lifting $\tilde{G}\left(N>n^{2}\right)$ of a fully-connected $4 \times n$ base graph $G$ :

$$
P=\left[\begin{array}{ccccc}
0 & 0 & 0 & \ldots & 0 \\
0 & 1 & 2 & \ldots & n-1 \\
0 & n & 2 n & \ldots & (n-1) n \\
0 & n+1 & 2(n+1) & \ldots & (n-1)(n+1)
\end{array}\right]
$$

Then, $\tilde{G}$ has girth 8.

Proof. The proof is similar to that of Lemma 11. In particular, by considering any sub-matrix of $P$ which consists of 3 rows of $P$ (corresponding to 3 check nodes of the 
base graph), one can show that the permutation shift of any 6-cycle containing those check nodes is non-zero.

Similar to the constructions of Lemmas 11 and 12, one can have constructions with girth 8 for protographs with larger left degrees.

The following matrices represent the permutation matrices for left degrees 5 and 6 , respectively:

$$
\begin{gathered}
P=\left[\begin{array}{ccccc}
0 & 0 & 0 & \ldots & 0 \\
0 & 1 & 2 & \ldots & n-1 \\
0 & n & 2 n & \ldots & (n-1) n \\
0 & n+1 & 2(n+1) & \ldots & (n-1)(n+1) \\
0 & n^{2} & 2 n^{2} & \ldots & (n-1) n^{2}
\end{array}\right], \\
P=\left[\begin{array}{ccccc}
0 & 0 & 0 & \ldots & 0 \\
0 & 1 & 2 & \ldots & n-1 \\
0 & n & 2 n & \ldots & (n-1) n \\
0 & n+1 & 2(n+1) & \ldots & (n-1)(n+1) \\
0 & n^{2} & 2 n^{2} & \ldots & (n-1) n^{2} \\
0 & n^{2}+1 & 2\left(n^{2}+1\right) & \ldots & (n-1)\left(n^{2}+1\right)
\end{array}\right] .
\end{gathered}
$$

For the two cases, the lifting degrees need to be at least $(n-1) n^{2}+1$ and $(n-$ 1) $\left(n^{2}+1\right)+1$, respectively.

\subsection{More Numerical Results}

Example 10. Consider the $(2048,1723)$ rate- 0.8413 regular LDPC code adopted in IEEE 802.3an. This code is constructed using a fully-connected base graph of size $6 \times 32$ and a lifting degree of 64, and has girth 6. Table 5.1 shows the proposed lower bounds on lifting degree for different girth values of a graph cyclically lifted from a $6 \times 32$ fully-connected base graph. 
Table 5.1: Lower Bounds on Lifting Degree for Different Girths of a Graph Lifted from a $6 \times 32$ Fully-connected Base Graph.

\begin{tabular}{|c|c|}
\hline Girth & Lower Bound on Lifting Degree \\
\hline \hline 6 & 32 \\
\hline 8 & 155 \\
\hline 10 & 4961 \\
\hline
\end{tabular}

Based on Table 5.1, one can see that the code used in IEEE 802.3an has the highest possible girth for the chosen lifting degree.

Example 11. In this example, for fully-connected base graphs of different sizes, we compare the proposed lower bounds on the lifting degree to achieve a certain girth with the results reported in [99] and [9] (lifting degrees for the same base graph that result in the same girth) as well as those obtained by a heuristic search in Table 5.2. The results of [9] are stronger than those of [99] only for $g=10$, and are thus reported only for this case. Table 5.2 shows that the proposed lower bound is rather tight for base matrices of size $3 \times n$, or cases corresponding to $g=6$. Table 5.2 also demonstrates that in five cases the results obtained by the heuristic search are superior to those of [99].

Table 5.2: Proposed Lower Bound on Lifting Degree $N_{L B}$ Compared to the Results of [99] $\left(N_{[91]}\right)$, Results of [9] and Results Found by a Heuristic Search $\left(N_{H}\right)$ for Different Girths of Graphs Lifted from Fully-connected Base Graphs with Different Sizes.

\begin{tabular}{|c|c|c|c|c|c|c|c|c|c|c|}
\hline \multirow{2}{*}{$\begin{array}{c}\text { Size of } \\
\text { Base Matrix }\end{array}$} & \multicolumn{3}{|c|}{$g=6$} & \multicolumn{3}{c|}{$g=8$} & \multicolumn{4}{c|}{$g=10$} \\
\cline { 2 - 12 } & $N_{L B}$ & $N_{H}$ & $N_{[91]}$ & $N_{L B}$ & $N_{H}$ & $N_{[91]}$ & $N_{L B}$ & $N_{H}$ & $N_{[91]}$ & $N_{[9]}$ \\
\hline \hline $3 \times 4$ & 4 & 5 & 5 & 7 & 9 & 9 & 37 & 39 & 39 & 37 \\
\hline $3 \times 5$ & 5 & 5 & 5 & 9 & 13 & 13 & 61 & 67 & 73 & 61 \\
\hline $3 \times 6$ & 6 & 7 & 7 & 11 & 18 & 18 & 91 & 113 & 123 & 101 \\
\hline $4 \times 5$ & 5 & 5 & 5 & 13 & 22 & 22 & 61 & 179 & 223 & - \\
\hline $4 \times 6$ & 6 & 7 & 7 & 16 & 28 & 29 & 91 & 287 & 383 & - \\
\hline
\end{tabular}




\section{Chapter 6}

\section{Finding Dominant Trapping Sets of Regular LDPC Codes}

\subsection{Introduction}

In this chapter, we study the problematic graphical structures that dominate the error floor performance of regular LDPC codes, collectively referred to as trapping sets, and demonstrate that they all contain at least one short cycle. By examining the relationships between cycles and trapping sets, we devise an efficient algorithm to find dominant trapping sets of a regular LDPC code.

\subsection{Development of the Proposed Algorithm}

In this section, we develop the proposed algorithm, and discuss its strengths and limitations. We start by a careful examination of the structure of dominant trapping sets followed by the detailed description and the pseudo-code of the proposed search algorithm. We then discuss the strengths and limitations of the algorithm in finding the dominant trapping sets. We continue this section with discussions on the inputs of the algorithm (initial trapping sets) as well as on its computational complexity and memory requirements. The section is ended with modifications required for the algorithm to search for non-elementary trapping sets. 


\subsubsection{Graphical Structure of Trapping Sets}

Without loss of generality, we assume that the induced subgraph of a trapping set is connected. Disconnected trapping sets can be considered as the union of connected ones. Moreover, to the best of our knowledge, almost all the structures reported as dominant trapping sets (of regular LDPC codes) in the literature have the property that every variable node is connected to at least two satisfied check nodes in the induced subgraph. We thus focus on trapping sets with this property except for irregular LDPC codes, where we relax this condition for degree-2 variable nodes. As an example, the subgraph in Figure 6.1 does not satisfy this condition. (Variable nodes and check nodes are represented by circles and squares, respectively.) Removal of node $v_{1}$ and its edges however makes the subgraph satisfy the condition. The following lemma proves this property for certain absorbing and ZP trapping sets.

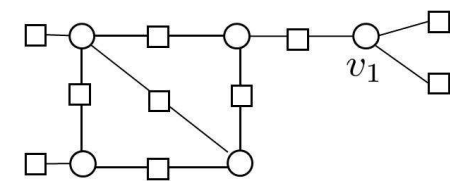

Figure 6.1: The induced subgraph of a trapping set.

Lemma 13. Suppose that $\mathcal{S} \subset L$ is an absorbing set (ZP trapping set) in $G=$ $(L \cup R, E)$, and that for all variable nodes $v \in \mathcal{S}$, we have $d(v) \geq 2(d(v) \geq 3)$. Then each variable node $v \in \mathcal{S}$ is connected to at least two satisfied check nodes in $G(\mathcal{S})$.

Proof. The proof follows from the definition of absorbing and ZP trapping sets.

For small trapping sets, which dominate the error floor performance, it is unlikely to see check nodes of degree larger than 2 in their subgraphs, i.e., most of the dominant trapping sets are elementary [16], [86]. Related to this, almost all the trapping sets reported as the dominant trapping sets of practical LDPC codes are elementary. In fact, it can be shown that the sizes of non-elementary trapping sets for left-regular graphs are generally larger than those of elementary ones (cf. Lemma 22 in Appendix C).

Example 12. Consider left-regular LDPC codes with left degree 4 and girths 6,8 and 10. Such codes can have elementary trapping sets of size 5, 8 and 17, respectively. The lower bounds on the sizes of non-elementary trapping sets $\mathcal{S}$ with less than 3 
unsatisfied check nodes and with at least one satisfied check node of degree larger than 2 in $G(\mathcal{S})$, for these codes, are however 7, 14 and 22, respectively (based on Lemma 22 in Appendix $C$, and by choosing $b=2)$. Moreover, for the same conditions, the minimum sizes of non-elementary trapping sets $\mathcal{S}$ with at least one unsatisfied check node of degree larger than 1 in $G(\mathcal{S})$ (and without satisfied check nodes of degree larger than 2 in $G(\mathcal{S})$ ) are at least 5, 11 and 17 , respectively.

In the following, we develop our search algorithm mainly for elementary trapping sets, and then present simple modifications to tailor the algorithm to find nonelementary trapping sets.

In the rest of the thesis, we use the notation $\mathcal{T}$ to denote the set of all trapping sets $\mathcal{S}$ in a graph $G$ whose induced subgraph $G(\mathcal{S})$ is connected and for which every node $v \in \mathcal{S}$ is connected to at least two nodes in $\Gamma_{\mathrm{e}}(\mathcal{S})$. It should be noted that $\mathcal{T}$ contains both elementary and non-elementary trapping sets. Notation $\mathcal{T}^{a}$ is used for the set of all elements in $\mathcal{T}$ with size $a$ and $\mathcal{T}_{\mathcal{S}}$ denotes the set of all elements in $\mathcal{T}$ that contain the set $\mathcal{S}$. Naturally, $\mathcal{T}_{\mathcal{S}}^{a}$ denotes the set of all elements in $\mathcal{T}$ of size $a$ that contain the set $\mathcal{S}$. In the following, we also assume that the Tanner graph $G$ has no parallel edges and no node of degree less than 2 .

\subsubsection{Expansion of Elementary Trapping Sets}

The main idea of the proposed algorithm is to start from a relatively small set of small elementary trapping sets, which are easy to enumerate, and then recursively expand them to larger elementary trapping sets. To achieve this, we first characterize the expansion of an elementary trapping set to a larger elementary trapping set through the following lemmas.

Lemma 14. Let $\mathcal{S}$ be an elementary trapping set of size a in $\mathcal{T}$. Then for each elementary trapping set $\mathcal{S}^{\prime} \in \mathcal{T}_{\mathcal{S}}^{a+1}$ (if any), the variable node in $\mathcal{S}^{\prime} \backslash \mathcal{S}$ is only connected to unsatisfied check nodes of $\mathcal{S}$ (i.e., to the check nodes in $\Gamma_{\mathrm{o}}(\mathcal{S})$ ).

Proof. If the node in $\mathcal{S}^{\prime} \backslash \mathcal{S}$ is connected to any satisfied check nodes of $\mathcal{S}$, then $\mathcal{S}^{\prime}$ will have unsatisfied check nodes in $\Gamma_{\mathrm{o}}\left(\mathcal{S}^{\prime}\right)$ connected to 3 variable nodes of $\mathcal{S}^{\prime}$. This contradicts $\mathcal{S}^{\prime}$ being an elementary trapping set.

Figure 6.2(a) depicts an example of the set $\mathcal{S}^{\prime}$ discussed in Lemma 14. (It should 


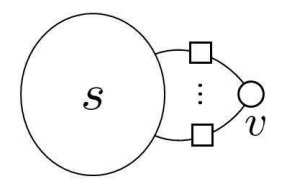

(a)

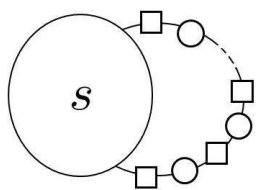

(b)

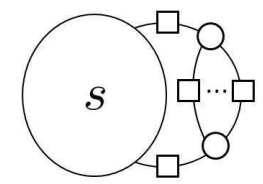

(d)

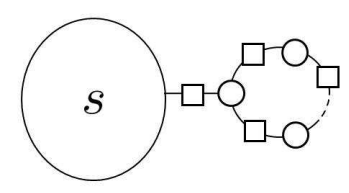

(c)

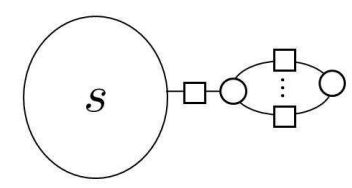

(e)

Figure 6.2: Possible expansions of an elementary trapping set $\mathcal{S}$ to a larger elementary trapping set $\mathcal{S}^{\prime}$. (Unsatisfied check nodes of $G\left(\mathcal{S}^{\prime}\right)$ are not shown.)

be noted that in all the configurations of Figure 6.2, including 6.2(a), unsatisfied check nodes of $G\left(\mathcal{S}^{\prime}\right)$ are not shown.)

Lemma 15. Suppose that $A=\left\{a_{1}, \ldots, a_{i}, a_{i+1}, \ldots, a_{I}\right\}$ is the sorted set of sizes of the elementary trapping sets in $\mathcal{T}$ in increasing order, where $a_{I}$ is the maximum size of such trapping sets. Let $\mathcal{S}$ be an elementary trapping set of size $a_{i}$ in $\mathcal{T}$. If $a_{i+1}>a_{i}+2$, then for each elementary trapping set $\mathcal{S}^{\prime} \in \mathcal{T}_{\mathcal{S}}^{a_{i+1}}$ (if any), the set $\mathcal{S}^{\prime} \backslash \mathcal{S}$ is connected to zero satisfied check nodes of $\mathcal{S}$ (i.e., nodes in $\Gamma_{\mathrm{e}}(\mathcal{S})$ ) and to only one or two unsatisfied check nodes of $\mathcal{S}$ (i.e., nodes in $\Gamma_{\mathrm{o}}(\mathcal{S})$ ). If the set $\mathcal{S}^{\prime} \backslash \mathcal{S}$ is connected to two nodes in $\Gamma_{\mathrm{o}}(\mathcal{S})$, then there is no cycle in $G\left(\mathcal{S}^{\prime} \backslash \mathcal{S}\right)$. If the set $\mathcal{S}^{\prime} \backslash \mathcal{S}$ is connected to only one node in $\Gamma_{\mathrm{o}}(\mathcal{S})$, then there is exactly one cycle in $G\left(\mathcal{S}^{\prime} \backslash \mathcal{S}\right)$.

Proof. If any variable node in $\mathcal{S}^{\prime} \backslash \mathcal{S}$ is connected to $\Gamma_{\mathrm{e}}(\mathcal{S})$, then $G\left(\mathcal{S}^{\prime}\right)$ contains satisfied check nodes of degree 4 or higher, or unsatisfied check nodes of degree greater than 1 . Both are in contradiction with $\mathcal{S}^{\prime}$ being an elementary trapping set.

Since $\mathcal{S}^{\prime}$ is an elementary trapping set, there cannot be more than one connection between each node of $\Gamma_{\mathrm{o}}(\mathcal{S})$ and the nodes in $\mathcal{S}^{\prime} \backslash \mathcal{S}$. To see this, consider a node $v \in \mathcal{S}^{\prime} \backslash \mathcal{S}$ which is connected to $\Gamma_{\mathrm{o}}(\mathcal{S})$. Node $v$ can have only one connection to $\Gamma_{\mathrm{o}}(\mathcal{S})$ because otherwise, all the other nodes in $\mathcal{S}^{\prime} \backslash \mathcal{S}$ can be removed and we will end up with an elementary trapping set of size $a_{i}+1$ in $\mathcal{T}$, which is in contradiction with the assumption of the lemma. 
Now suppose that there are at least 3 connections between the variable nodes in $\mathcal{S}^{\prime} \backslash \mathcal{S}$ and $\Gamma_{\mathrm{o}}(\mathcal{S})$. Based on the discussion in the previous paragraph, this means that there are at least 3 variable nodes in $\mathcal{S}^{\prime} \backslash \mathcal{S}$ each with a single connection to a different check node in $\Gamma_{\mathrm{o}}(\mathcal{S})$. If $G\left(\mathcal{S}^{\prime} \backslash \mathcal{S}\right)$ is not connected, then one can remove one of its components and obtain an elementary trapping set of size smaller than $a_{i+1}$, which results in a contradiction. If $G\left(\mathcal{S}^{\prime} \backslash \mathcal{S}\right)$ is connected, then one can find the shortest paths in $G\left(\mathcal{S}^{\prime} \backslash \mathcal{S}\right)$ between every two variable nodes of $\mathcal{S}^{\prime} \backslash \mathcal{S}$ that are connected to $\Gamma_{\mathrm{o}}(\mathcal{S})$, and among them select the one with the least number of nodes. By keeping the nodes on the selected path and removing all the other nodes in $\mathcal{S}^{\prime} \backslash \mathcal{S}$, one can then obtain an elementary trapping set of size smaller than $a_{i+1}$, which is again a contradiction. We therefore conclude that the number of connections between the variable nodes in $\mathcal{S}^{\prime} \backslash \mathcal{S}$ and $\Gamma_{\mathrm{o}}(\mathcal{S})$ must be strictly less than 3.

For the case that $\mathcal{S}^{\prime} \backslash \mathcal{S}$ is connected to exactly two nodes in $\Gamma_{\mathrm{o}}(\mathcal{S})$, there must be two different variable nodes $v$ and $v^{\prime}$ of $\mathcal{S}^{\prime} \backslash \mathcal{S}$ corresponding to those connections. Also, there must be no cycles in $G\left(\mathcal{S}^{\prime} \backslash \mathcal{S}\right)$. Otherwise, one can remove all the variable nodes on the cycle except those on the shortest path between $v$ and $v^{\prime}$, and obtain an elementary trapping set larger than $a_{i}$ but smaller than $a_{i+1}$. This contradicts the lemma's assumption. Figure 6.2(b) is an example of the case where $\mathcal{S}^{\prime} \backslash \mathcal{S}$ is connected to exactly two nodes in $\Gamma_{\mathrm{o}}(\mathcal{S})$.

The proof for the case with one connection is similar and omitted. Figure 6.2(c) is an example of this case, where the expansion of the set $\mathcal{S}$ is through a lollipop walk. In both Figures 6.2(b) and $(c)$, the dashed line indicates that more variable and check nodes can be part of the chain.

Lemma 16. Suppose that $A=\left\{a_{1}, \ldots, a_{i}, a_{i+1}, \ldots, a_{I}\right\}$ is the sorted set of sizes of the elementary trapping sets in $\mathcal{T}$ in increasing order and that $a_{i+1}=a_{i}+2$. Let $\mathcal{S}$ be an elementary trapping set of size $a_{i}$ in $\mathcal{T}$. If the girth of the graph is larger than 4 , then for each elementary trapping set $\mathcal{S}^{\prime} \in \mathcal{T}_{\mathcal{S}}^{a_{i+1}}$ (if any), the only possible configuration for $G\left(\mathcal{S}^{\prime}\right)$ is that of Figure 6.2(b), described in Lemma 15, with only 2 variable nodes in $\mathcal{S}^{\prime} \backslash \mathcal{S}$. If the girth is 4 , then the only possible configurations are those in Figures 6.2(b) (with only 2 variable nodes in $\left.\mathcal{S}^{\prime} \backslash \mathcal{S}\right)$ 6.2(d) and 6.2(e).

Proof. The proof is similar to that of Lemma 15 and is omitted. 


\subsubsection{Proposed Algorithm}

The basic idea behind the proposed algorithm is to construct larger elementary trapping sets by expanding smaller ones. More precisely, given an elementary trapping set $\mathcal{S}$ of size $a_{i}$ at the input, the algorithm finds all the elementary trapping sets $\mathcal{S}^{\prime}$ containing $\mathcal{S}$, with the property that their size $a_{i+1}$ is the smallest size greater than $a_{i}$. The algorithm then continues by using the sets found in the current step as the inputs to the next step and finds the next set of larger elementary trapping sets. Each step of the algorithm is performed by using Lemmas $14-16$. The pseudo-code for one step of the proposed algorithm is given in Algorithm 2.

Algorithm 2 : Expansion of input elementary trapping sets to larger ones of size up to $k$ with the number of unsatisfied check nodes up to $T$ in $G=(L \cup R, E)$. $\left(\mathcal{L}_{\text {in }}\right.$ and $\mathcal{L}_{\text {out }}$ are the lists of input and output trapping sets, respectively.)

1: Inputs: $G, \mathcal{L}_{\text {in }}, k$ and $T$.

2: Initialization: $\mathcal{L}_{\text {out }} \leftarrow \emptyset$.

3: repeat

4: $\quad$ Select an element of $\mathcal{L}_{\text {in }}$ and denote it as $\mathcal{S}$.

5: Construct a new graph $G^{\prime}$ by removing all the nodes in $\Gamma_{\mathrm{e}}(\mathcal{S})$, their adjacent edges and their neighbors from $G$.

6: $\quad i_{\max } \leftarrow(k-|\mathcal{S}|)$ and $\mathcal{G} \leftarrow \emptyset$.

7: $\quad$ for each node $c$ in $\Gamma_{\mathrm{o}}(\mathcal{S})$ do

8: $\quad$ Examine the neighborhood of $c$ in $G^{\prime}$ one layer at a time and to the maximum of $i_{\max }$ layers in search for paths with $i \leq i_{\max }$ variable nodes between $c$ and the other nodes of $\Gamma_{\mathrm{o}}(\mathcal{S})$, and lollipop walks with $i \leq i_{\max }$ variable nodes starting from $c$.

9: $\quad$ Denote $\mathcal{G}_{c}$ as the set of all such paths/lollipop walks of shortest length $i$ (if any).

10: $\quad$ if $i<i_{\max }$ then

11: $\quad i_{\max } \leftarrow i$.

12: $\mathcal{G} \leftarrow \mathcal{G}_{c}$.

13: $\quad$ else

14: $\mathcal{G} \leftarrow \mathcal{G} \cup \mathcal{G}_{c}$. 


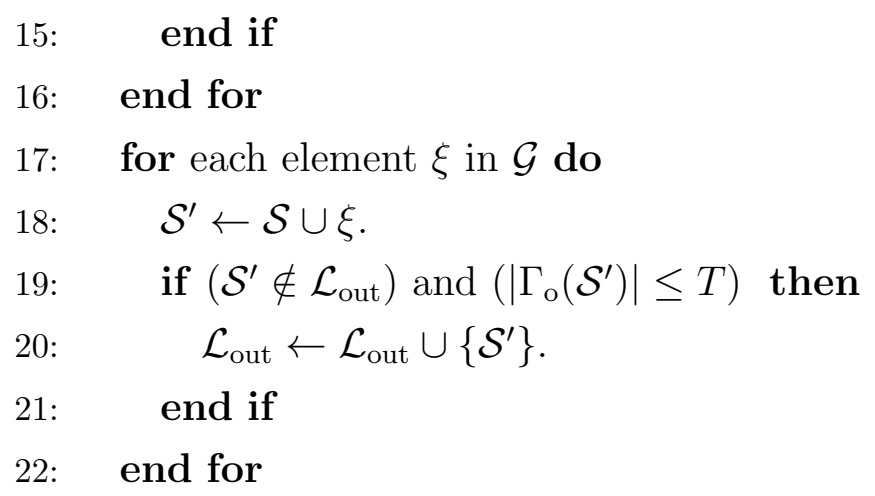

23: until all the elements of $\mathcal{L}_{\text {in }}$ are selected.

24: Output: $\mathcal{L}_{\text {out }}$.

Remark 2. Note that in Line 5 of Algorithm 2, all the satisfied check nodes in $G(\mathcal{S})$, i.e., the set $\Gamma_{\mathrm{e}}(\mathcal{S})$, and their neighboring variable nodes are removed from the graph. This is because, based on Lemmas 14 - 16, such nodes cannot be part of the expansion of an elementary trapping set.

Remark 3. In Line 19 of the algorithm, the threshold value $T$ on the number of unsatisfied check nodes is needed to keep the complexity of the overall search algorithm, which involves multiple applications of Algorithm 2, low. A proper choice of T has negligible effect on the ability of the algorithm to find the larger trapping sets $(a, b)$ with small values of $b$. This is explained in the following example.

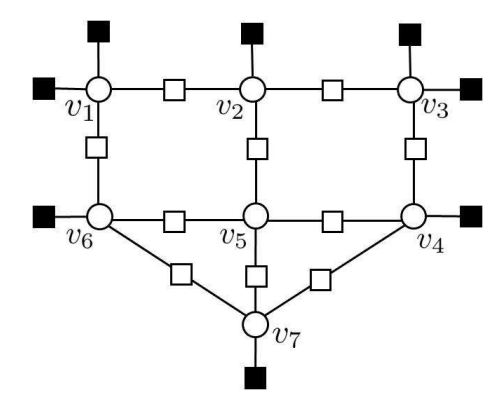

Figure 6.3: An example of a $(7,8)$ trapping set (satisfied and unsatisfied check nodes are shown by empty and full squares, respectively).

Example 13. Consider the $(7,8)$ trapping set $\mathcal{S}$, shown in Figure 6.3. This set belongs to $\mathcal{T}$ and contains 4 trapping sets of size 6 , all also in $\mathcal{T}$. These four trapping sets can each be obtained by removing one of the nodes $v_{1}, v_{3}, v_{5}$ or $v_{7}$. As a result, we 
have $(6,8),(6,8),(6,12)$ or $(6,10)$ trapping sets, respectively. Among these trapping sets, the $(6,8)$ ones have a smaller number of unsatisfied check nodes. Starting from each of these two trapping sets, Algorithm 2 finds $\mathcal{S}$. Hence, ignoring the $(6,12)$ (or even $(6,12)$ and $(6,10))$ trapping set(s) does not impair the ability of the algorithm to find $\mathcal{S}$.

Remark 4. Based on Lemmas 14 - 16, it can be proved that starting from an $(a, b)$ elementary trapping set $\mathcal{S}$, Algorithm 2 will find all the $\left(a^{\prime}, b^{\prime}\right)$ elementary trapping sets of the smallest size $a^{\prime}$ larger than a that contain $\mathcal{S}$ (this requires the removal of the condition $\left|\Gamma_{\mathrm{o}}\left(\mathcal{S}^{\prime}\right)\right| \leq T$ in Line 19). Note that this does not imply that by the recursive application of Algorithm 2 one can obtain all the elementary trapping sets containing $\mathcal{S}$. The following example demonstrates this.

Example 14. Consider the $(6,6)$ elementary trapping set $\mathcal{S}^{\prime}=\left\{v_{1}, v_{2}, v_{3}, v_{4}, v_{5}, v_{6}\right\}$ in Figure 6.4. Assume that Algorithm 2 starts from the elementary trapping set $\mathcal{S}=\left\{v_{1}, v_{2}, v_{6}\right\}$. Using this input, the output of the algorithm is $\left\{v_{1}, v_{2}, v_{6}, v_{5}\right\}$. By subsequent applications of the algorithm, the next outputs are $\left\{v_{1}, v_{2}, v_{6}, v_{5}, v_{7}\right\}$ and $\left\{v_{1}, v_{2}, v_{6}, v_{5}, v_{7}, v_{3}, v_{4}\right\}$, respectively. This means that the algorithm does not find the trapping set $\mathcal{S}^{\prime}$, although $\mathcal{S}^{\prime}$ contains $\mathcal{S}$. (It is however easy to see that if the algorithm starts from the set $\left\{v_{2}, v_{3}, v_{4}, v_{5}\right\}$, it will find $\mathcal{S}^{\prime}$.)

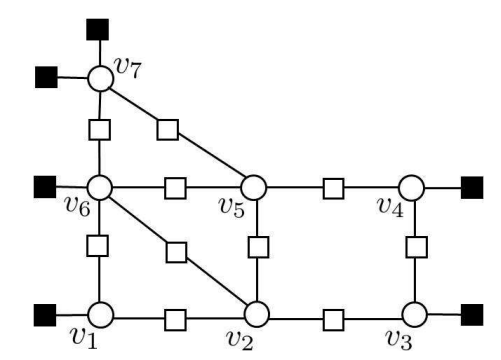

Figure 6.4: An example explaining that the algorithm cannot find all the elementary trapping sets containing a specific elementary trapping set.

In fact, the sufficient condition for the algorithm to find a trapping set $\mathcal{S}^{\prime}$ of size $a_{j}$, starting with one of its subsets $\mathcal{S}$ of size $a_{i}<a_{j}$, is that $\mathcal{S}^{\prime}$ has at least one subset in $\mathcal{T}_{\mathcal{S}}^{a}$ for all $a \in A, a_{i}<a<a_{j}$, where $A$ is defined in Lemma 15.

The following example shows that despite the limitation explained in Remark 4 and Example 14, for many cases, the proposed algorithm in fact finds (in a guaranteed fashion) all the trapping sets $(a, b)$ with $a$ and $b$ up to certain values. 
Example 15. For a left-regular graph with left degree 4 and girth larger than 6, initiating the proposed algorithm with the set of cycles of length $g$ and $g+2$, one can guarantee to find all the elementary trapping sets of size less than 9 with less than 5 unsatisfied check nodes. This can be seen by the inspection of all the possible structures for such trapping sets and verifying that for each structure, the removal of only one variable node will result in another trapping set in $\mathcal{T}$. Subsequent removals of such nodes from an $(a, b)$ elementary trapping set with $a<9$ and $b<5$ in $\mathcal{T}$, thus, results in a sequence of embedded elementary trapping sets in $\mathcal{T}$, each with size only one less than that of its parent. The sequence will always end with a cycle of length $g$ or $g+2$. This implies that all such trapping sets satisfy the sufficient condition, mentioned earlier, for being found by the algorithm starting from a cycle of length $g$ or $g+2$.

Similar results to those of Example 15 can be found for other left-regular graphs. This is the subject of Chapter 8. For irregular graphs however, it is very difficult to provide such guarantees. This is due to the fact that the number of possible structures for a trapping set of a given size could be very large in this case.

Remark 5. For irregular $L D P C$ codes, the variable nodes with large degrees cannot be part of small trapping sets. This is formulated in the following lemma.

Lemma 17. In a graph $G$ with girth $g>4$, if an $(a, b)$ trapping set $\mathcal{S}$ contains a variable node $v$ of degree $d(v)>b$, then $a \geq d(v)+1-b$.

Proof. Consider the $d(v)$ neighbors of $v$ in $G(\mathcal{S})$. At least $d(v)-b$ of them are in $\Gamma_{\mathrm{e}}(\mathcal{S})$ and are thus connected to other variable nodes in $\mathcal{S}$. None of such variable nodes can share more than one check node from $\Gamma_{\mathrm{e}}(\mathcal{S})$ with $v$, because of the condition $g>4$. This implies that there are at least $d(v)-b$ variable nodes in $\mathcal{S} \backslash\{v\}$.

Based on Lemma 17, for example, for an irregular code with girth larger than 4, a variable node of degree 15 cannot participate in an $(a, b)$ trapping set with $a<13$ and $b<4$. Such results can be used to simplify the algorithm by removing the large degree variable nodes and their edges from the graph.

Remark 6. It is easy to see that for the left-regular graphs with left degree 3 or 4, all the trapping sets found by Algorithm 2 are ZP trapping sets. For the left-regular graphs with left degree 3 , the obtained trapping sets are also absorbing sets. 
Remark 7. Our simulations for many practical LDPC codes show that in almost all the cases, $a_{i+1} \leq a_{i}+3$, where $a_{i}$ is the size of the initial elementary trapping set and $a_{i+1}$ is the size of the smallest elementary trapping set containing the initial one.

In the following, we discuss the selection of the initial set of elementary trapping sets.

\subsubsection{Initial Set of Trapping Sets}

One of the graphical objects that plays an important role in the structure of trapping sets is a cycle. Tian et al. [105] showed that every stopping set $^{1}$ includes the variable nodes of at least one cycle. Related to this, the induced graph of the support of a pseudo-codeword always contains at least one cycle [60]. In [16], [121], [128], it was shown that an overwhelming majority of dominant trapping (absorbing) sets are combinations of short cycles. Short cycles are also easy to enumerate [121]. We thus use short cycles as the initial inputs to the proposed algorithm. The following lemma provides more justifications for this choice.

\section{Lemma 18.}

i) In a left-regular graph $G$ with left degree $d_{l} \geq 2$, if the induced subgraph $G(\mathcal{S})$ of an $(a, b)$ trapping set $\mathcal{S}$ does not contain any cycle, then $b \geq a\left(d_{l}-2\right)+2$. The inequality is satisfied with equality for elementary trapping sets.

ii) The variable nodes in any shortest cycle (of length g) of a Tanner graph form an elementary trapping set.

iii) Let $\mathcal{T}$ be the set of all trapping sets $\mathcal{S}$ of a graph $G$, whose induced subgraph $G(\mathcal{S})$ is connected and for which every node $v \in \mathcal{S}$ is connected to at least two nodes in $\Gamma_{\mathrm{e}}(\mathcal{S})$ (see Section II for the definition of $\Gamma_{e}(\mathcal{S})$ ). Then for every $\mathcal{S} \in \mathcal{T}$, its induced subgraph $G(\mathcal{S})$ contains at least one cycle.

iv) Suppose that $\mathcal{S} \subset L$ is an absorbing set of a left-regular Tanner graph $G=$ $(L \cup R, E)$ with left node degrees at least 2. Then $G(\mathcal{S})$ contains at least one cycle.

\footnotetext{
${ }^{1}$ Stopping sets are the problematic structures for iterative decoding of LDPC codes over the binary erasure channel (BEC) [18].
} 
v) Suppose that $\mathcal{S} \subset L$ is a ZP trapping set of a Tanner graph $G=(L \cup R, E)$ with node degrees at least 3. Then $G(\mathcal{S})$ contains at least one cycle.

Proof. The proof of Part (i) is provided in Appendix D. The proofs for Parts (ii) and (iii) are simple and thus omitted. Parts (iv) and (v) follow from Lemma 13 and Part (iii).

It can be shown that Part (i) of Lemma 18 can be generalized to the case where the variable node degree distribution is irregular. In this case, the result is modified as $b \geq a\left(\bar{d}_{\mathcal{S}}-2\right)+2$, where $\bar{d}_{\mathcal{S}}$ is the average degree of variable nodes in $\mathcal{S}$. The following example, based on Part (i) of Lemma 18, demonstrates that cycle-free $(a, b)$ trapping sets have relatively large values of $b$.

Example 16. For a left-regular graph $G$ with left degree 4 , any cycle-free $(a, b)$ trapping set satisfies $b \geq 2(a+1)$. Such large values of $b$ for a given a would imply that the $(a, b)$ trapping set is not dominant. ${ }^{2}$

Our simulation results indicate that for denser graphs, the set of short cycles of length $g$, or $g$ and $g+2$, where $g$ is the girth, is sufficient to find almost all the small (with, say, $a \leq 10$ ) dominant trapping sets. In this case, adding short cycles of larger lengths to the input set has negligible effect on the performance of the algorithm, while increasing its complexity. For example, we examined a number of randomly constructed codes with rates larger than 0.4. The codes had block length 1000 and left-regular Tanner graphs with left degree 5 and girth 6 . In all cases, the trapping sets obtained by Algorithm 2 using cycles of length 6 and 8 as the input set were identical to those obtained by using cycles of length 6,8 and 10 as the input set.

For sparser graphs, however, one may need to use short cycles of larger lengths (e.g., $g, g+2$, and $g+4$ ) as the initial set.

\subsubsection{Complexity of the Algorithm}

The complexity of the algorithm is highly dependent on the short cycle distribution of the graph, which itself is mostly a function of the degree distribution of the graph (see Chapter 3). As a result, in general, the complexity increases much faster with the increase in the average variable and check node degrees of the graph than it does with increasing the block length. To have a more detailed analysis of the complexity

\footnotetext{
${ }^{2}$ For dominant trapping sets, the value of $b$ is usually close to, or smaller than, the value of $a$.
} 
of Algorithm 2, we note that the total complexity can be divided into two parts: $a$ ) Finding the initial input set and $b$ ) Expanding the input set to larger trapping sets.

Regarding the complexity of Part $(a)$, assuming that an exhaustive brute-force search is used to find cycles of length $k$, say for $g \leq k \leq g+4$, the complexity is $O\left(n d_{v}^{k / 2} d_{c}^{k / 2}\right)$, for a $\left(d_{v}, d_{c}\right)$ regular graph with $n$ variable nodes. This is obtained by considering all the possible paths of length $k$ starting from all the $n$ variable nodes in the graph. The memory required for the storage of all the $k$-cycles is of order $O\left(k N_{k}\right)$, where $N_{k}$ is the number of $k$-cycles in the graph. To the best of our knowledge, there is no theoretical result on how $N_{k}$ scales with $n$ or the degree distribution of the Tanner graph. Empirical results in Chapter 3 however suggest that $N_{k}$ is mainly a function of the degree distribution and is rather independent of $n$.

Regarding the complexity of expanding the input trapping sets to larger ones, consider the expansion of an $(a, b)$ trapping set $\mathcal{S}$ of a $\left(d_{v}, d_{c}\right)$ graph. Depending on the size $a^{\prime}>a$ of the smallest trapping $\operatorname{set}(\mathrm{s}) \mathcal{S}^{\prime}$ that contain $\mathcal{S}$, the complexity and memory requirements for finding and storing the sets $\mathcal{S}^{\prime}$ would differ. For $a^{\prime}=a+1, a+2$ and $a+3$, the complexity is $O\left(b d_{c}\right), O\left(b d_{v} d_{c}^{2}\right)$ and $O\left(b d_{v}^{2} d_{c}^{3}\right)$, respectively. The memory requirement for these cases are, respectively, $O\left(a b d_{c}\right), O\left(a b d_{v} d_{c}^{2}\right)$ and $O\left(a b d_{v}^{2} d_{c}^{3}\right)$. To see this, for example, consider the case where $a^{\prime}=a+1$. To find $\mathcal{S}^{\prime}$, one needs to check at most $b\left(d_{c}-1\right)$ variable nodes as possible candidates, which corresponds to $O\left(b d_{c}\right)$ complexity. ${ }^{3}$ The memory required to store all possible trapping sets of size $a+1$ obtained through such a search is thus upper bounded by $(a+1) b d_{c}$, which is of order $O\left(a b d_{c}\right)$.

Based on the above discussions, assuming that the initial set is limited to cycles of length up to $g+4$, and that we only consider trapping sets $\mathcal{S}^{\prime}$ of size up to $a^{\prime}=a+3$ in the expansion process, the complexity of Algorithm 2 is $O\left(d_{v}^{2} d_{c}^{3}\left(T \sum_{i=g / 2}^{g / 2+2} N_{2 i}+\right.\right.$ $\left.n d_{v}^{g / 2} d_{c}^{g / 2-1}\right)$ ) and the memory requirement is $O\left(T d_{v}^{2} d_{c}^{3} \sum_{i=g / 2}^{g / 2+2} 2 i N_{2 i}\right)$, where $T$ is the maximum number of unsatisfied check nodes in Algorithm 2. It is however important to note that the actual complexity and memory requirements are much less than what these complexity bounds may suggest. In particular, our simulation results show that codes with block lengths up to about 10,000 with a wide variety of degree distributions can be managed using the proposed algorithm on a regular desktop computer.

\footnotetext{
${ }^{3}$ Here, we have ignored the extra complexity that would be involved in attaching the new variable node to the existing trapping set $\mathcal{S}$ to form (and to store) $\mathcal{S}^{\prime}$.
} 


\subsubsection{Expansion of Non-Elementary Trapping Sets}

According to the general definition of trapping sets, any arbitrary set of variable nodes can be considered as a trapping set. Hence, to expand a connected trapping set $\mathcal{S}$ of size $a$, one just needs to select a variable node from the neighboring variable nodes, and add it to $\mathcal{S}$ to obtain a new trapping set $\mathcal{S}^{\prime}$ with size $a^{\prime}=a+1$. This method of expansion leads to an exponentially growing search space. Even by limiting the search space to the trapping sets in $\mathcal{T}$, i.e., connected trapping sets for which every variable node is connected to at least two satisfied check nodes, there are still too many configurations for $\mathcal{S}^{\prime}$, especially when $a^{\prime} \gg a$. For practical LDPC codes with $g>4$, however, considering a nested sequence of trapping sets in $\mathcal{T}$, the size of the next larger trapping set $a^{\prime}$ is almost always less than $a+3$.

The search for non-elementary trapping sets of size $a^{\prime} \leq a+3$ in a graph with girth $g>4$, can be performed similar to what was described for the elementary trapping sets with a number of small differences. For non-elementary trapping sets, since there is no limitation on the degrees of the check nodes in $G(\mathcal{S})$, only the variable nodes of $\mathcal{S}$ and their edges are removed from the graph. Then the shortest paths between different check nodes of $G(\mathcal{S})$ or the shortest lollipop walks starting from different check nodes of $G(\mathcal{S})$ are found. However, it should be mentioned that not all such structures will necessarily satisfy the condition that each variable node is connected to at least two satisfied check nodes. After finding a candidate trapping set, one should thus check for this condition. In summary, to find the non-elementary trapping sets of size $a^{\prime} \leq a+3$, the only modifications needed to be applied to Algorithm 2 are the followings:

5: Construct a new graph $G^{\prime}$ by removing all the nodes of $\mathcal{S}$ and their adjacent edges from $G$.

7: for each node $c$ in $\Gamma(\mathcal{S})$ do

8: Examine the neighborhood of $c$ in $G^{\prime}$ one layer at a time and to the maximum of $i_{\max }$ layers in search for paths with $i \leq i_{\max }$ variable nodes between $c$ and the other nodes of $\Gamma(\mathcal{S})$, and lollipop walks with $i \leq i_{\max }$ variable nodes starting from c.

19: if $\left(\mathcal{S}^{\prime} \in \mathcal{T}\right)$ and $\left(\mathcal{S}^{\prime} \notin \mathcal{L}_{\text {out }}\right)$ and $\left(\left|\Gamma_{\mathrm{o}}\left(\mathcal{S}^{\prime}\right)\right| \leq T\right)$ then 


\subsection{Numerical Results}

We have applied the proposed algorithm successfully to a large number of regular LDPC codes. Here, we only present the results for four of them. The first three examples are random and structured LDPC codes whose dominant trapping sets have already been reported in the literature and thus provide us with a reference for comparison. The fourth example is a random LDPC code of rate $1 / 2$ with variable node degree 4 . To verify the trapping sets found by the proposed algorithm for this code, we estimate the error floor using importance sampling based on the obtained trapping sets and demonstrate that the estimation is practically identical to the results of Monte Carlo simulations. The reported running times in the following examples are for a desktop computer with 2-GHz CPU and 1 GB of RAM.

Example 17. We consider an LDPC code constructed by the progressive edge growth (PEG) algorithm [39] (PEGReg252x504 of [132]). This code is left-regular with the left degree 3 , and girth 8 . The same code was also investigated in [67] and the distribution of its fully absorbing sets were determined. For Algorithm 2, the short cycles of length $g, g+2$ and $g+4$ were used as the initial input set. The algorithm was limited to find trapping sets of maximum size 13, and the threshold $T$ was selected such that only the two most dominant trapping sets for each size were considered. (Using a larger $\mathrm{T}$ has no effect on the accuracy of the results.) Since all the variable nodes have degree 3, all the trapping sets found by Algorithm 2 are absorbing sets. Fully absorbing sets were found by examining the obtained absorbing sets and testing them for the definition of a fully absorbing set. Table 6.1 shows the absorbing sets and the fully absorbing sets found by the proposed algorithm and their multiplicities. In the table, we have also reported the results obtained by the exhaustive search algorithm of [67], for comparison. As can be seen from Table 6.1, for many classes of trapping sets, the proposed algorithm found exactly the same number of fully absorbing sets as the exhaustive search algorithm of [67] did. For the other classes, the difference between the two sets of results is rather small. Moreover, the proposed algorithm found $(11,3),(13,3),(10,4)$ and $(12,4)$ fully absorbing sets which are out of the reach of the exhaustive search algorithm. It is worth mentioning that the exhaustive search algorithm of [67] took about 7 hours to find only the first three rows of Table 6.1 [67] (needless to say, the larger the size of the absorbing sets, the longer the running time of the algorithm). This is while Algorithm 2 took only 
Table 6.1: Dominant Absorbing Sets(ABS) and Fully Absorbing Sets of the $P E$ GReg252x504 Code Obtained by the Proposed Algorithm and the Exhaustive Search Algorithm of [67]

\begin{tabular}{|c|c|c|c|}
\hline $\begin{array}{c}\text { Trapping } \\
\text { Set }\end{array}$ & $\begin{array}{c}\text { Proposed } \\
\text { Algorithm } \\
\text { (ABS) }\end{array}$ & $\begin{array}{c}\text { Proposed } \\
\text { Algorithm } \\
\text { (Fully ABS) }\end{array}$ & $\begin{array}{l}\text { Exhaustive } \\
\text { Search [67] } \\
\text { (Fully ABS) }\end{array}$ \\
\hline$(4,4)$ & 802 & 760 & 760 \\
\hline$(5,3)$ & 14 & 14 & 14 \\
\hline$(5,5)$ & 11279 & 10156 & 10156 \\
\hline$(6,4)$ & 985 & 849 & 849 \\
\hline$(6,6)$ & 86391 & 66352 & 66352 \\
\hline$(7,3)$ & 57 & 47 & 47 \\
\hline$(7,5)$ & 27176 & 21810 & 22430 \\
\hline$(8,2)$ & 5 & 4 & 4 \\
\hline$(8,4)$ & 2610 & 2258 & 2270 \\
\hline$(9,1)$ & 1 & 1 & 1 \\
\hline$(9,3)$ & 156 & 146 & 146 \\
\hline$(10,2)$ & 6 & 6 & 6 \\
\hline$(10,4)$ & 7929 & 6691 & - \\
\hline$(11,3)$ & 605 & 558 & - \\
\hline$(12,2)$ & 25 & 24 & 26 \\
\hline$(12,4)$ & 23668 & 19959 & - \\
\hline$(13,1)$ & 1 & 1 & 1 \\
\hline$(13,3)$ & 2124 & 1954 & - \\
\hline
\end{tabular}

10 minutes to find all the absorbing sets listed in Table 6.1.

Example 18. In this example, we consider the Tanner $(155,64)$ code [100]. This code was also investigated in [114]. The exhaustive search algorithm of [114] showed that this code has no trapping set of length less than 8 with 2 unsatisfied check nodes and has no trapping set of length up to 11 with 1 unsatisfied check node. It was also 
Table 6.2: Dominant Trapping Sets of the Tanner $(155,64)$ Code Obtained by the Proposed Algorithm

\begin{tabular}{|c|c|}
\hline Trapping Set & Multiplicity \\
\hline \hline$(4,4)$ & 465 \\
\hline$(5,3)$ & 155 \\
\hline$(6,4)$ & 930 \\
\hline$(7,3)$ & 930 \\
\hline$(8,2)$ & 465 \\
\hline$(9,3)$ & 1395 \\
\hline$(10,2)$ & 1395 \\
\hline$(11,3)$ & 1860 \\
\hline$(12,2)$ & 930 \\
\hline
\end{tabular}

shown in [114] that the code has $465(8,2)$ trapping sets.

The girth for the Tanner graph of this code is $g=8$. The short cycles of length $g, g+2$ and $g+4$ were used as the initial inputs to Algorithm 2. The algorithm was limited to only find trapping sets of maximum size 12 and the threshold $T$ was selected such that only the two most dominant trapping sets for each size were considered. Table 6.2 shows the trapping sets found by the proposed algorithm and their multiplicity. As can be seen in the table, the algorithm found all the $465(8,2)$ trapping sets among others. All the trapping sets in Table 6.2 were found in less than 2 minutes. To further verify that the obtained trapping sets do in fact include the dominant ones, we performed Monte Carlo simulations on the code with a 4-bit quantized min-sum decoder over the AWGN channel at signal-to-noise ratio (SNR) of $6.5 \mathrm{~dB}$ (which is in the error floor region of this code). Among the 300 error patterns, about $90 \%$ were $(8,2)$ trapping sets, about $8 \%$ were $(10,2)$ trapping sets, and only 2 did not belong to the sets reported in Table 6.2.

Example 19. As the third example, we consider the Margulis $(2640,1320)$ code [75], [132]. It is known that the most dominant trapping sets of this code are $1320(12,4)$ and $1320(14,4)$ trapping sets [86]. The Tanner graph of this code has girth $g=8$. The set of short cycles of length $g, g+2$ and $g+4$ was used as the input 
Table 6.3: Dominant Trapping Sets of the Margulis $(2640,1320)$ Code Obtained by the Proposed Algorithm and the Algorithm of [4]

\begin{tabular}{|l|c||l|c|}
\hline $\begin{array}{l}\text { Trapping } \\
\text { Set }\end{array}$ & $\begin{array}{c}\text { Proposed } \\
\text { Algorithm } \\
\text { (Absorbing) }\end{array}$ & $\begin{array}{l}\text { Proposed } \\
\text { Algorithm } \\
\text { (Trapping) }\end{array}$ & $\begin{array}{c}\text { Algorithm } \\
\text { of [4] } \\
\text { (Trapping) }\end{array}$ \\
\hline \hline$(7,5)$ & 7920 & 7920 & - \\
\hline$(8,6)$ & 106920 & $>106920$ & - \\
\hline$(9,5)$ & 2640 & 2640 & - \\
\hline$(10,6)$ & 117480 & $>117480$ & - \\
\hline$(11,5)$ & 5280 & 5280 & 9 \\
\hline$(12,4)$ & 1320 & 1320 & 1320 \\
\hline$(13,5)$ & 2640 & 26400 & 2699 \\
\hline$(14,4)$ & 1320 & 1320 & 1320 \\
\hline$(15,5)$ & 0 & 26400 & 7938 \\
\hline$(16,6)$ & 0 & 258347 & 21153 \\
\hline$(17,5)$ & 5280 & 5280 & 0 \\
\hline$(18,6)$ & 0 & 132000 & 2642 \\
\hline
\end{tabular}

set of the proposed algorithm. The algorithm was limited to use only the two most dominant trapping sets for each size. Since the degree of all the variable nodes of this code is 3, all the trapping sets found by Algorithm 2 are also absorbing sets. The first column in Table 6.3 shows the dominant absorbing sets found by Algorithm 2. For comparison, the dominant trapping sets obtained by the algorithm of [4] are listed in the last column of Table 6.3. It should be noted that in [4] there is no condition on the number of satisfied check nodes connected to each variable node. Thus to have a fair comparison, we also consider the trapping sets constructed by the combination of trapping sets found by Algorithm 2 and one of their neighboring variable nodes. The second column of Table 6.3 shows the number of such trapping sets ${ }^{4}$. As can be seen, for all the trapping set classes, the proposed algorithm performs at least as well

\footnotetext{
${ }^{4}$ Our simulations indicate that the effect of extra trapping sets found by removing the constraint on the number of satisfied check nodes connected to each variable node of the trapping set on the error floor performance of the code is negligible.
} 
as the algorithm of [4]. Moreover, the required time for the algorithm of [4] was 7 days on a $2.8 \mathrm{GHz}$ PC [4], while the proposed algorithm took about 5 hours to finish. As another comparison for the running time of the proposed algorithm, it took the algorithm 55 minutes to find all the dominant absorbing sets of size less than 15, while the same task took 8.2 hours for the impulse method of [16] on a comparable computer (2.2-GHz CPU with 1 GB RAM).

Example 20. For this example, we consider a $(1008,504)$ random code with variable node degree 4 and check node degree 8 constructed by the program of [132]. ${ }^{5}$ This code has one cycle of length $4\left(C_{4}\right)$. In addition to that, the short cycles of length 6 to 10 were used as the initial input set for Algorithm 2. The algorithm was constrained to find dominant trapping sets of size up to 12 and to use only the two most dominant trapping sets for each size. Table 6.4 shows the dominant trapping sets found by Algorithm 2 and their multiplicities. It is worth mentioning that none of the trapping sets listed in Table 6.4 contains any of the variable nodes participating in $C_{4}$. The trapping sets reported in Table 6.4 were used to estimate the error floor of the code using the importance sampling technique described in [16].

Table 6.4: Dominant Trapping Sets of the $(1008,504)$ Regular LDPC Code $\left(d_{v}=4, d_{c}=\right.$ 8) Obtained by the Proposed Algorithm

\begin{tabular}{|c|c|}
\hline Trapping Set & Multiplicity \\
\hline \hline$(5,6)$ & 15 \\
\hline$(6,4)$ & 1 \\
\hline$(6,6)$ & 36 \\
\hline$(7,5)$ & 13 \\
\hline$(8,6)$ & 5 \\
\hline$(9,6)$ & 5 \\
\hline$(10,6)$ & 3 \\
\hline$(11,6)$ & 3 \\
\hline$(12,8)$ & 75 \\
\hline
\end{tabular}

Figure 6.5 shows the Monte Carlo simulation results for the frame error rate

\footnotetext{
${ }^{5}$ Using code6. $c$ with seed $=380$.
} 
(FER) and the corresponding error floor estimation based on importance sampling. The results are for a 3-bit min-sum decoder with maximum number of iterations 50. As can be seen in Figure 6.5, the estimation closely matches the Monte Carlo simulation, verifying the dominance of the trapping sets found by Algorithm 2. Monte Carlo simulations also revealed that the most harmful trapping set of this code is the $(6,4)$ trapping set. In fact, in almost all the decoding failures, the decoder converged to the $(6,4)$ trapping set. As can be seen in Table 6.4, all the trapping sets have at least 4 unsatisfied check nodes. This makes the exhaustive search methods of [113], [114], [67] ineffective for finding the dominant trapping sets of this code. This is while all the trapping sets in Table 6.4 were found in less than 5 minutes by the proposed algorithm.

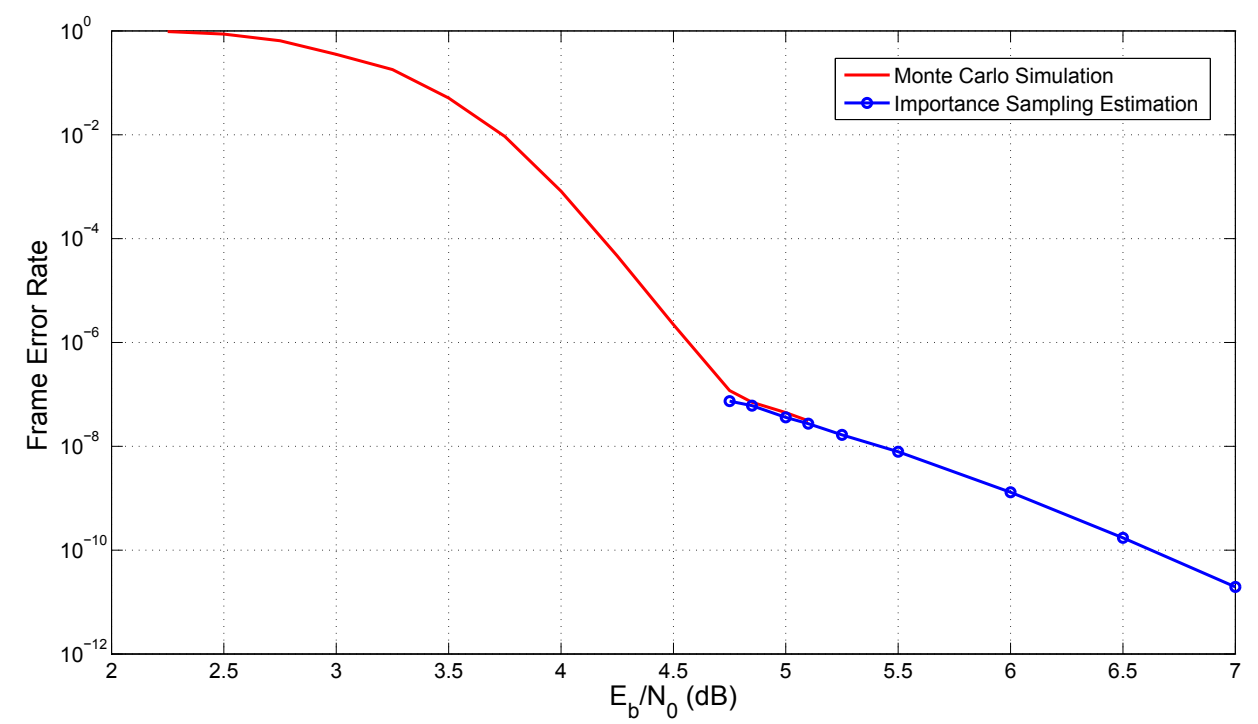

Figure 6.5: Error floor estimation and Monte Carlo simulation for the $(1008,504)$ regular LDPC code $\left(d_{v}=4, d_{c}=8\right)$. 


\section{Chapter 7}

\section{Finding Dominant Trapping Sets of Irregular LDPC Codes}

\subsection{Introduction}

In this chapter, we study the trapping set structures that dominate the error floor performance of irregular LDPC codes, and generalize the work of previous chapter to be also applicable to irregular codes. For the irregular LDPC codes which do not have variable nodes of degree 2, Algorithm 2 without any modification can be used to find the dominant trapping sets. As mentioned in Remark 5 (Chapter 6, Section 6.2.3), based on the desired sizes of trapping sets, one may also remove the high degree variable nodes and their edges from the graph to simplify the algorithm. In the case that the code has variable nodes of degree 2, some modifications are needed for the initial input set of the algorithm. In this chapter, we study the effect of degree-2 variable nodes on the structure of trapping sets in irregular LDPC codes, and present simple steps to find the corresponding trapping sets.

\subsection{On the Degree-2 Variable Nodes}

It is known that degree-2 variable nodes play an important role in the performance of irregular LDPC codes. On the one hand, to have codes with asymptotic performance close to the capacity, the proportion of degree- 2 variable nodes should be as large as possible. This is usually a considerable fraction of the total variable nodes of the code. On the other hand, having a large proportion of degree-2 variable nodes results in a small minimum distance and a high error floor [106]. Cycles containing only 
degree-2 variable nodes are codewords. Hence, to have a large minimum distance, it is desirable to avoid such cycles, especially the shorter ones. To avoid all cycles of any length containing only degree- 2 variable nodes, the number of these nodes $n_{v_{2}}$ must be strictly less than the number of check nodes $m$ (i.e., $n_{v_{2}}<m$ ). Based on this fact, a class of irregular LDPC codes with $n_{v_{2}}=m-1$, called extended irregular repeat accumulate (eIRA) codes was proposed in [126]. It was shown in [126] that these codes exhibit relatively better error floor performance compared to the codes constructed by the optimized degree distributions without applying this restriction on $n_{v_{2}}$. Related to this, it was proved in [82] that for the case where $n_{v_{2}}>m$, the minimum distance grows at most logarithmically with the code length. For the special case where $n_{v_{2}}=m$ and all the degree- 2 variable nodes are part of a single cycle, the minimum distance is a sub-linear power function of the block length [106]. In the following, we study the effect of having a large fraction of degree- 2 variable nodes on the structure of trapping sets in irregular LDPC codes.

Example 21. For all the degree distributions optimized for rate-1/2 LDPC codes on the binary-input AWGN (BIAWGN) channel [85], 43\% to 55\% of variable nodes are of degree 2. This implies that, on average, every check node in the corresponding codes is connected to about 2 variable nodes of degree 2.

The average number of degree-2 variable nodes connected to each check node becomes even larger for the optimized codes of higher rate. This is explained in the next example.

Example 22. For the optimized degree distribution of rate $8 / 9$ over the BIAWGN channel with the maximum variable node degree 10 [85], 31\% of variable nodes are of degree 2. This implies that, on average, every check node in a Tanner graph with this degree distribution is connected to about 6 variable nodes of degree 2.

Consequently, it is very likely to see chains of degree-2 variable nodes, referred to as 2-chains, in the Tanner graph of LDPC codes with optimized degree distributions. The length of a 2-chain is defined as the number of the edges in the subgraph induced by the degree-2 variable nodes of the chain. That is, the length of a 2-chain containing $k$ variable nodes of degree 2 is $2 k$. A 2 -chain of length $2 k$ is a $(k, 2)$ trapping set (with the exception of the case where the chain is closed and forms a cycle; in that case, we refer to the 2-chain as a 2-cycle. A 2-cycle of length $2 k$, is a $(k, 0)$ trapping set). Having only 2 unsatisfied check nodes, 2 -chains of length $2 k$ are among the most 
dominant trapping sets of size $k$. Figure $7.1(a)$ shows a 2-chain of length $10($ a $(5,2)$ trapping set). Note that this trapping set also contains two $(4,2)$, three $(3,2)$, four $(2,2)$ and five $(1,2)$ trapping sets as its subsets. It is worth noting that although for the cases where $n_{v_{2}}=m-1$ and $n_{v_{2}}=m$, the graph may have no or only one 2-cycle, it can have many 2-chains of different lengths. For example, it is easy to see that for the case where $m=n_{v_{2}}$ and all the degree- 2 variable nodes are contained in a single cycle, there are $m$ 2-chains of length $2 k, 1 \leq k \leq m-1$.

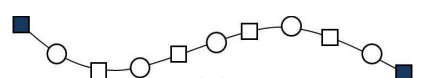

(a)

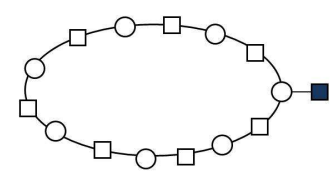

(b)

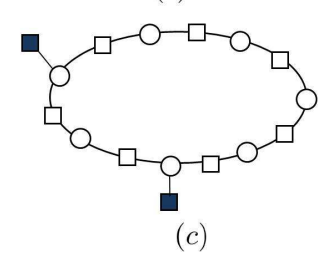

Figure 7.1: Typical trapping sets constructed mostly by the degree-2 variable nodes.

Another aspect of having 2-chains in the Tanner graph of irregular LDPC codes is that they might participate in short cycles with other variable nodes of higher degrees. These cycles have low approximate cycle extrinsic message degree (ACE) (ACE is defined as $\sum_{i} d_{i}-2$, where the summation is taken over all the variable nodes of the cycle, and $d_{i}$ is the degree of the $i$ th variable node in the cycle [105]). It has been shown that cycles with low ACE deteriorate the error rate performance, and that avoiding them in the construction of irregular LDPC codes generally improves the error rate [118], [112].

Example 23. Consider the case where $m=n_{v_{2}}$ and all the degree-2 variable nodes are contained in a single cycle. In this case, there exist two 2-chains between any two check nodes of the graph. This implies that every variable node of degree $d_{v}>2$ along with the 2-chains connecting its check nodes form several trapping sets with at most $d_{v}-2$ unsatisfied check nodes.

Example 24. Figure 7.1(b) shows a $(7,1)$ trapping set composed of one variable node of degree 3 and a chain of six variable nodes of degree 2. 
Example 25. The $(12,1)$ trapping sets of the $(1944,972)$ LDPC code adopted in the IEEE 802.11 standard [41] are single cycles of length 24, each consisting of a 2-chain of length 22 and one degree-3 variable node.

Even in the cases where $n_{v_{2}}<m$ (but not much smaller), it is likely to see cycles mostly constructed by 2 -chains.

Example 26. Figure 7.1(c) shows a $(7,2)$ trapping set composed of two variable nodes of degree 3 and five variable nodes of degree 2.

Due to the important role that 2-chains (and 2-cycles) play in the formation of dominant trapping sets, we study the necessary conditions to avoid these structures in the following theorem.

Theorem 4. Let $m$ be the number of check nodes and $n_{v_{2}}$ be the number of degree-2 variable nodes in the graph $G$ corresponding to an irregular code $\mathcal{C}$. If for all $k \geq 2$, $G$ has no 2-chains of length longer than or equal to $2 k$ and no 2-cycles of length less than or equal to $2 k$ then

$$
m \geq n_{v_{2}}\left(1+\frac{1}{\sum_{i=0}^{k-2}\left(d_{c, \max }-1\right)^{\left\lfloor\frac{i+1}{2}\right\rfloor}}\right),
$$

where $d_{c, \text { max }}$ is the maximum check node degree in $G$.

Proof. Let $G_{v_{2}}$ denote the induced subgraph of degree-2 variable nodes of the graph $G$. This subgraph contains no cycle. Otherwise, the length of such a cycle would be at least $2 k+2$, which would imply the existence of a 2-chain of length $2 k$ in $G_{v_{2}}$, and thus in $G$. This contradicts the assumption of the theorem. The subgraph $G_{v_{2}}$ is thus composed of some tree-like components. For each component, the number of check nodes is always larger than the number of variable nodes by one. Therefore the total number of check nodes of the graph is more than the number of degree- 2 variable nodes by at least the number of disjoint components in $G_{v_{2}}$ (some check nodes of $G$ may not appear in $G_{v_{2}}$ ). To avoid 2-chains of length $2 k$ or longer, the maximum number of variable nodes in each component is $\sum_{i=0}^{k-2}\left(d_{c, \text { max }}-1\right)^{\left\lfloor^{\left.\frac{i+1}{2}\right\rfloor}\right.}$ (Appendix E, Lemma 23). The minimum number of components in $G_{v_{2}}$ is thus $\left\lceil n_{v_{2}} / \sum_{i=0}^{k-2}\left(d_{c, \text { max }}-1\right)^{\left\lfloor\frac{i+1}{2}\right\rfloor}\right\rceil$.

Theorem 4 can be used to determine the maximum number of degree- 2 variable nodes in an irregular graph to avoid 2-chains (and 2-cycles) of a specific length. 
Example 27. For an irregular code with 1000 check nodes of degree $d_{c}=6$, to avoid $(4,2)$ trapping sets corresponding to 2-chains of length 8, the number of variable nodes of degree 2 must be at most 910.

Theorem 4 can be also used to obtain some information about the existence of trapping sets in a code.

Example 28. For the same scenario as that of Example 27 (i.e., $m=1000, d_{c}=6$ ), the eIRA construction [126] results in $n_{v_{2}}=m-1=999$. For these parameters, the smallest value of $k$ which satisfies the inequality of Theorem 1 is $k=9$. This implies that the eIRA code will have 2-chains of length 16 and smaller, corresponding to $(k, 2)$ trapping sets for all values of $k<9$.

\subsection{Finding Trapping Sets of Irregular LDPC Codes}

In this section, we present a simple process to find the dominant trapping sets involving degree-2 variable nodes. The process can be used in combination with Algorithm 2 to find the dominant trapping sets of irregular graphs containing degree-2 variable nodes. It is important to note that according to the definition of absorbing sets, any variable node of degree 2 in these sets is connected to 2 satisfied check nodes. Also, for the trapping sets found by Algorithm 2, each variable node is connected to at least 2 satisfied check nodes. Therefore, 2-chains and other trapping sets containing variable node(s) of degree 2 with one satisfied check node are neither absorbing sets nor found by Algorithm 2. In fact, it appears that being connected to 2 satisfied check nodes is too strong of a condition for a variable node of degree 2 to be part of a dominant trapping set. For this reason, we consider also trapping sets whose variable nodes of degree 2 are connected to only one satisfied check node. To obtain such trapping sets using the expansion of smaller trapping sets, we consider an $(a-1, b)$ trapping set $\mathcal{S}$ which is expanded to a trapping set $\mathcal{S}^{\prime}$ by the connection of a variable node $v$ of degree 2 to an unsatisfied check node of $\mathcal{S}$. Three cases are possible:

a) $v$ is not connected to any other check node of $\Gamma(\mathcal{S})$. In this case, $\mathcal{S}^{\prime}=\mathcal{S} \cup\{v\}$ is an $(a, b)$ trapping set. If $\mathcal{S}$ is elementary, so is $\mathcal{S}^{\prime}$.

b) $v$ is also connected to a satisfied check node of $\mathcal{S}$. In this case, $\mathcal{S}^{\prime}=\mathcal{S} \cup\{v\}$ is an $(a, b)$ non-elementary trapping set. 
c) $v$ is also connected to another unsatisfied check node of $\mathcal{S}$. In this case, $\mathcal{S}^{\prime}=$ $\mathcal{S} \cup\{v\}$ is an $(a, b-2)$ trapping set. If $\mathcal{S}$ is elementary (or is in the set $\mathcal{T}$ ), so is $\mathcal{S}^{\prime}$.

Such an expansion of a trapping set can be performed multiple times by adding one neighboring variable node of degree 2, each time. This is summarized in Algorithm 3. In general, Algorithm 3 can be used with Algorithm 2 to expand the trapping sets found by Algorithm 2. This is summarized in Algorithm 4.

Algorithm 3: Finding trapping sets of size up to $k$ with the number of unsatisfied check nodes up to $T$ constructed by adding degree- 2 variable nodes to the input trapping sets for an irregular LDPC code with the Tanner graph $G=(L \cup R, E)$. $\left(\mathcal{L}_{\text {in }}\right.$ and $\mathcal{L}_{\text {out }}$ are the lists of input and output trapping sets, respectively. $)$

1: Inputs: $G, \mathcal{L}_{\text {in }}, k$ and $T$.

2: $\mathcal{L}_{\text {out }} \leftarrow \emptyset$.

3: repeat

4: $\quad$ Select an element of $\mathcal{L}_{\text {in }}$ with size less than $k$, and denote it as $t$.

5: Form the set $N_{2}(t)$ which contains variable nodes of degree 2 in $L \backslash t$ that are connected to at least one unsatisfied check node of $t$, i.e., to $\Gamma_{\mathrm{o}}(t)$.

6: for each node $v$ in $N_{2}(t)$ do

7: $\quad t^{\prime} \leftarrow t \cup\{v\}$.

8: $\quad$ if $\left(t^{\prime} \in \mathcal{T}\right)^{1}$ and $\left(t^{\prime} \notin \mathcal{L}_{\text {out }}\right)$ and $\left(\left|\Gamma_{\mathrm{o}}\left(t^{\prime}\right)\right| \leq T\right)$ then

9: $\quad \mathcal{L}_{\text {out }} \leftarrow \mathcal{L}_{\text {out }} \cup\left\{t^{\prime}\right\}$.

10: $\quad$ end if

11: $\quad$ end for

12: until all the elements of $\mathcal{L}_{\text {in }}$ are selected.

13: Output: $\mathcal{L}_{\text {out }}$.

Remark 8. Note that in Algorithm 3, the number of unsatisfied check nodes of the resultant trapping sets never increases. Hence, to find trapping sets of size a with less than $b$ unsatisfied check nodes, one should consider all the $\left(a^{\prime}, b^{\prime}\right)$ trapping sets with $a^{\prime}<a, b^{\prime}<b .^{2}$ It should be mentioned that since every single variable node of degree

\footnotetext{
${ }^{1}$ This condition ensures that each variable node of degree larger than 2 is connected to at least 2 satisfied check nodes. The condition has no bearing on degree-2 variable nodes.

${ }^{2}$ Although this condition may not cover all the trapping sets discussed in Part $c$ of Section IV.B,
} 
$d_{v}$ can be regarded as a $\left(1, d_{v}\right)$ trapping set, to find the trapping sets with less than $b$ unsatisfied check nodes, we consider also all the variable nodes of degree $d_{v} \leq b$ as part of the initial set. For example, for the case of $b=3$, starting with a single variable node of degree $d_{v}=2$ or $d_{v}=3$, two typical structures of the resultant trapping sets are shown in Figures 7.2(a) and 7.2(b), respectively. Note that starting from a degree-2 variable node and performing the above steps results in finding a 2-chain.

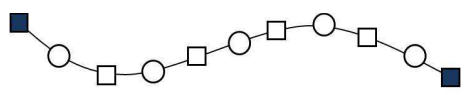

(a)

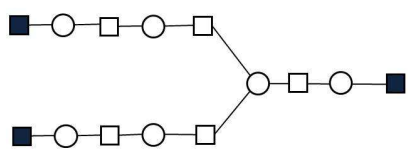

(b)

Figure 7.2: Typical expansions of degree-2 and degree-3 variable nodes by adding the neighboring degree- 2 variable nodes.

Algorithm 4: Finding trapping sets of size up to $k$ with the number of unsatisfied check nodes up to $T$ for an irregular LDPC code with the Tanner graph $G=(L \cup$ $R, E)$.

$\left(\mathcal{L}_{\text {in }}\right.$ and $\mathcal{L}_{\text {out }}$ are the lists of input and output trapping sets, respectively.

1: Inputs: $G, \mathcal{L}_{\text {in }}, k$ and $T$.

2: Use $\mathcal{L}_{\text {in }}$ as the input of Algorithm 2

3: $\mathcal{L} 1_{\text {out }}=$ trapping sets found by Algorithm 2

4: $\mathcal{L} 2_{\text {in }}=\mathcal{L} 1_{\text {out }} \bigcup\{$ low degree variable nodes $\}$

5: Use $\mathcal{L} 2_{\text {in }}$ as the input of Algorithm 3

6: $\mathcal{L}_{\text {out }}=$ trapping sets found by Algorithm 3

7: Output: $\mathcal{L}_{\text {out }}$.

Remark 9. For irregular codes, in addition to short cycles, cycles with low ACE are our simulations show that for the tested codes, almost all the trapping sets are in fact found by Algorithm 3. The trapping sets that are missed by Algorithm 3 are the ones that can only be obtained by starting from trapping sets with larger number of unsatisfied check nodes. 
also considered as part of the initial input set of Algorithm 2. This is because these cycles may not be found using the expansion process of Algorithm 2. Algorithm 2 finds the smallest trapping sets containing the input, which are usually the combination of the input and a short cycle (or a structure described in Lemmas 2 - 4). Since variable nodes of large degree are more likely to be part of such structures, the outputs of Algorithm 2 are usually the combinations of the input and variable node(s) of large degree. This is while cycles with low ACE are generally constructed by low degree variable nodes. Cycles with low ACE can be easily found by monitoring the ACE value during the execution of a cycle finding algorithm.

Remark 10. As an alternative approach to using Algorithm 4, one can only use Algorithm 3 with the variable nodes of low degree and cycles with low ACE as the initial input set, and then recursively expand them to larger trapping sets. It should however be noted that for the irregular LDPC codes with a small fraction of degree-2 variable nodes, this approach may not find all the dominant trapping sets of the code.

\subsection{Numerical Results}

In this section, we present the results of applying the proposed algorithm to three irregular LDPC codes. To find the dominant trapping sets of the irregular codes, we used two approaches. In the first approach, we used Algorithms 2 and 3 in the framework described in Algorithm 4. In this approach, as the first step, we used the short cycles of the codes, as well as the low ACE cycles as the initial input set, and applied Algorithm 2. We then used the trapping sets found by Algorithm 2 along with the variable nodes of low degree, and applied Algorithm 3 to expand them. As the second approach, we only used the variable nodes of low degree and cycles with low ACE as the initial input set, and then used Algorithm 3 to recursively expand them to larger trapping sets. Interestingly, for all the three codes, the results of the second approach were very close to those of the first one.

Example 29. For this example, we consider the irregular LDPC code constructed by the PEG algorithm (PEGirReg252x504 code [132]). This code was also investigated in [67] for its fully absorbing sets. For Algorithm 2, the short cycles of length $g$, $g+2$, and the cycles with length less than 20 and ACE less than 4 were used as the initial input set. The algorithm was constrained to find only trapping sets of size 
less than 12 and the threshold $T$ was selected such that only the 4 most dominant trapping sets for each size were considered. The resultant trapping sets and variable nodes of degree 2 and 3 were then expanded by adding neighboring degree- 2 variable nodes, and finally were examined to find the fully absorbing sets. Table 7.1 shows the fully absorbing sets found by Algorithm 4 and the exhaustive search algorithm of [67]. It should be noted that, similar to [67], we relaxed the condition that degree-

Table 7.1: Dominant Fully Absorbing Sets of the PEGirReg252x504 Code Obtained by the Proposed Algorithm and the Algorithm of [67]

\begin{tabular}{|c|c|c|}
\hline $\begin{array}{c}\text { Trapping } \\
\text { Set }\end{array}$ & $\begin{array}{c}\text { Proposed } \\
\text { Algorithm }\end{array}$ & $\begin{array}{c}\text { Exhaustive } \\
\text { Search [67] }\end{array}$ \\
\hline \hline$(3,2)$ & 219 & 219 \\
\hline$(4,2)$ & 208 & 208 \\
\hline$(5,2)$ & 198 & 198 \\
\hline$(6,2)$ & 205 & 205 \\
\hline$(7,1)$ & 2 & - \\
\hline$(7,2)$ & 271 & 274 \\
\hline$(8,1)$ & 8 & - \\
\hline$(8,2)$ & 458 & 468 \\
\hline$(9,1)$ & 16 & - \\
\hline$(9,2)$ & 855 & - \\
\hline$(10,1)$ & 22 & - \\
\hline$(10,2)$ & 1533 & - \\
\hline$(11,1)$ & 36 & - \\
\hline
\end{tabular}

2 variable nodes of (fully) absorbing sets must be connected to two satisfied check nodes. As can be seen from Table 7.1, the proposed algorithm found almost all the fully absorbing sets of this code ${ }^{3}$. Moreover, the proposed algorithm found a number of $(a, 1)$ trapping sets for $a \geq 7$, which were not found by the algorithm of [67]. For the second approach, the cycles of length up to 20 with ACE lower than 4 and the

\footnotetext{
${ }^{3}$ The multiplicity for trapping sets $(7,1),(7,2),(8,1)$ and $(8,2)$ in the last column of Table 7.1 should be changed to $2,272,8$ and 460 , respectively [68].
} 
variable nodes of degree 2 and 3 were used as the initial inputs, and the algorithm found almost the same trapping sets as in the first approach. For the running time, the first and the second approaches took 15 minutes and 5 minutes, respectively.

Example 30. For this example, we used the $(1944,972)$ structured irregular code with rate 1/2, adopted in IEEE.802.11 standard [41]. We used the same parameters as in the previous example for the two approaches. Table 7.2 shows the number of dominant trapping sets of different sizes found by the algorithm of [4] and the proposed approaches. For this code, both of our approaches found exactly the same set of trapping sets. In fact, all the trapping sets listed in Table 7.2 have one of the following three structures: a 2-chain, a single cycle with low ACE, and the combination of a 2-chain and a single cycle of low ACE. For example, all the trapping sets of size less than 7 listed in Table 7.2 are 2 -chains, and all the $(12,1)$ trapping sets are single cycles of eleven degree- 2 variable nodes and one degree- 3 variable node. As can be seen in Table 7.2, for all classes of trapping sets, the proposed algorithms found at least as many trapping sets as the algorithm of [4] did. The first and the second approaches took 45 and 5 minutes, respectively, to find all the trapping sets in Table 7.2. This is while the algorithm of [4] took 5 days (on a 2.8-GHz CPU) to find the results reported in Table 7.2.

Based on the importance sampling technique of [16], the trapping sets in Table 7.2 with size $l, 6 \leq l \leq 12$, were used to estimate the error floor of this code for a 3-bit quantized min-sum decoder over the AWGN channel. Figure 7.3 shows the error floor estimation and the Monte Carlo simulation results for this code. As can be seen in Figure 7.3, the IS estimation closely matches the Monte Carlo simulation, further verifying the dominance of the trapping sets found by the proposed algorithm.

Example 31. As the last example, we use the following degree distribution optimized for the min-sum algorithm in [15] and construct a $(1000,499)$ LDPC code using the PEG algorithm: $\lambda(x)=.30370 x+.27754 x^{2}+.02843 x^{5}+.20014 x^{6}+.19019 x^{19}$ and $\rho(x)=.0160 x^{5}+.9840 x^{6}$. The girth of the resultant graph is 6 , and we use the short cycles of length 6 and 8, and cycles of length up to 20 with ACE less than 4 as the initial input set of Algorithm 3. It takes the algorithm 1 minute to find the trapping sets of size up to 10. Based on the obtained trapping sets and using the importance sampling, we estimate the error floor of the code. Figure 7.4 shows the 
Table 7.2: Dominant Trapping Sets of the (1944, 972) Code Obtained by the Proposed Algorithm

\begin{tabular}{|c|c|c|}
\hline $\begin{array}{c}\text { Trapping } \\
\text { Set }\end{array}$ & $\begin{array}{c}\text { Proposed } \\
\text { Algorithm }\end{array}$ & $\begin{array}{c}\text { Algorithm } \\
\text { of [4] }\end{array}$ \\
\hline \hline$(2,2)$ & 810 & - \\
\hline$(3,2)$ & 729 & - \\
\hline$(4,2)$ & 648 & 648 \\
\hline$(5,2)$ & 567 & 567 \\
\hline$(6,2)$ & 486 & 486 \\
\hline$(7,2)$ & 486 & 485 \\
\hline$(8,2)$ & 648 & 637 \\
\hline$(9,2)$ & 972 & - \\
\hline$(10,2)$ & 1377 & 1210 \\
\hline$(11,2)$ & 1944 & 1635 \\
\hline$(12,1)$ & 81 & 81 \\
\hline$(12,2)$ & 2754 & 2166 \\
\hline$(13,1)$ & 162 & 162 \\
\hline$(14,1)$ & 162 & 162 \\
\hline$(15,1)$ & 162 & - \\
\hline$(16,1)$ & 162 & - \\
\hline$(17,1)$ & 162 & - \\
\hline$(18,1)$ & 81 & \\
\hline
\end{tabular}

estimation and Monte Carlo simulations for this code. As can be seen in this figure, the estimation closely matches the Monte Carlo simulation results, verifying that the dominant trapping sets of the code have been found by the algorithm. 


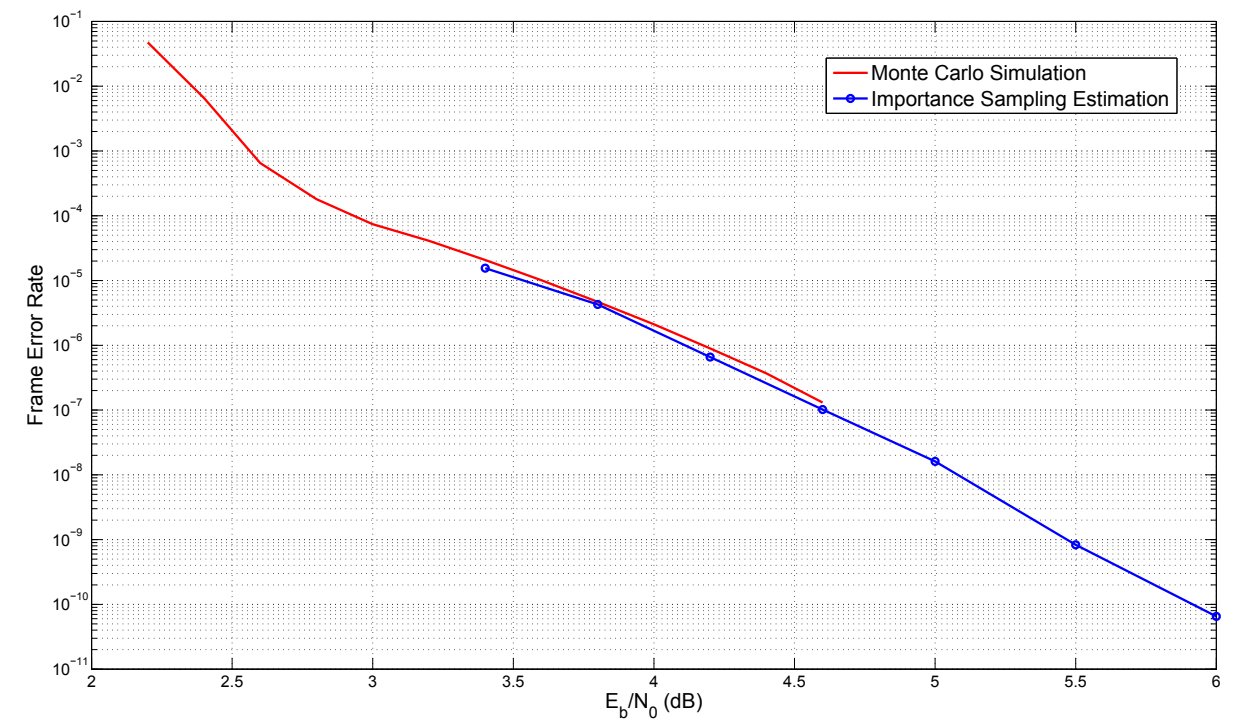

Figure 7.3: Error floor estimation and Monte Carlo simulation for the $(1944,972)$ irregular LDPC code.

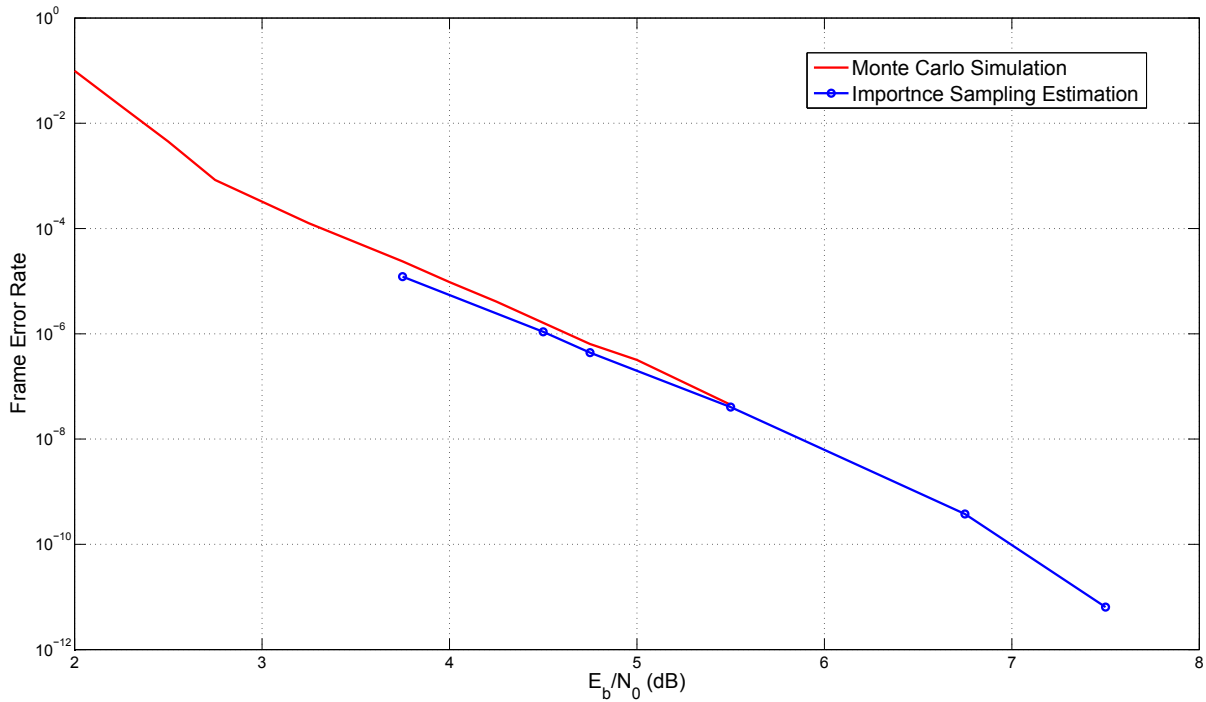

Figure 7.4: Error floor estimation and Monte Carlo simulation for the $(1000,499)$ irregular LDPC code. 


\section{Chapter 8}

\section{On Characterization of Elementary Trapping Sets of Left-Regular LDPC Codes}

In this chapter, for the category of variable-regular ${ }^{1}$ LDPC codes with a certain variable node degree and a given girth, we study the topological structure of $(a, b)$ elementary trapping sets (ETSs) for given values of $a$ and $b$, and find all the nonisomorphic structures of such ETSs. We present a simple property for characterization of dominant classes of ETSs based on short cycles in the Tanner graph of the code. We also present a simple search algorithm that starts from the short cycles of the graph and finds all the ETSs with such property in a guaranteed fashion.

\subsection{Non-isomorphic Structures of ETSs}

Elementary trapping sets $($ ETS) in left-regular Tanner graphs (which are also in $\mathcal{T}$ ) are the main focus of this chapter. To investigate the structure of ETSs of a certain $(a, b)$ class in left-regular Tanner graphs with left-degree $d_{l}$ and girth $g$, we need to obtain all the non-isomorphic graphical structures of such trapping sets. To simplify the representation of the subgraph induced by an ETS in a left-regular graph, we often use an alternate graphical representation called normal graphs [61]. The normal graph of an ETS is obtained from the induced subgraph of the set by removing all the degree- 1 check nodes and their edges from the subgraph, and by replacing each

\footnotetext{
${ }^{1}$ It is worth noting that any regular LDPC code is also a variable-regular LDPC code. Regular LDPC codes are of particular importance due to their simpler decoder implementation and superior error floor performance compared to irregular codes.
} 
degree-2 check node with an edge.

Example 32. Figures 8.1(a) and 8.1(b) represent the induced subgraph and the normal graph of a $(5,4)$ ETS in a left-regular graph with $d_{l}=4$ and $g=6$, respectively. In Figure 8.1(a), and the rest of this chapter, variable nodes are represented by circles, and satisfied and unsatisfied check nodes are shown by empty and full squares, respectively.

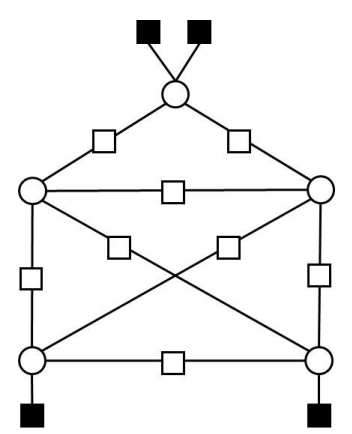

(a)

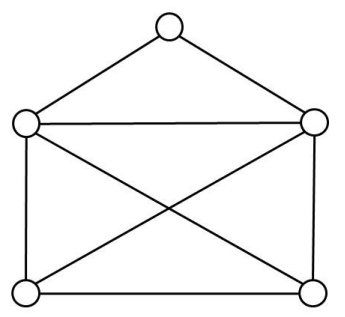

(b)

Figure 8.1: A $(5,4)$ elementary trapping set and its normal graph.

It is easy to see that there is a one-to-one correspondence between an ETS of a left-regular graph and its normal graph. Given $d_{l}$, one can construct the subgraph of the ETS from a given normal graph by replacing each edge $\{u, v\}$ of the normal graph with two edges $\{u, c\}$ and $\{c, v\}$, where $c$ is a degree-2 check node which is also added to the graph, and by connecting $d_{l}-d(v)$ check nodes of degree one to every variable node $v$ with $d(v)<d_{l}$.

In the following, we provide an example to demonstrate how all the non-isomorphic structures of a class of ETSs can be found for rather small values of $d_{l}, a$ and $b$.

Proposition 5. Any $(6,2)$ ETS of a left-regular LDPC code with $d_{l}=4$ and $g=6$ has one of the structures presented in Figure 8.2.

Proof. We use the normal graph representation, and prove that any $(6,2)$ ETS in a left-regular LDPC code with $d_{l}=4$ and $g=6$ has one of the normal graph representations given in Figure 8.3. 


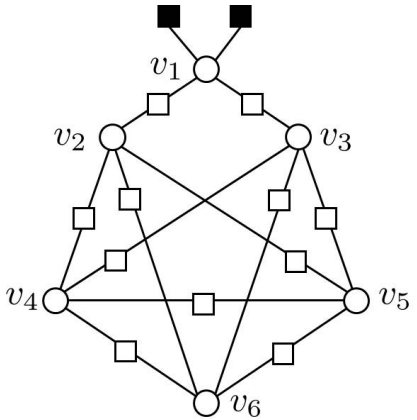

(a)

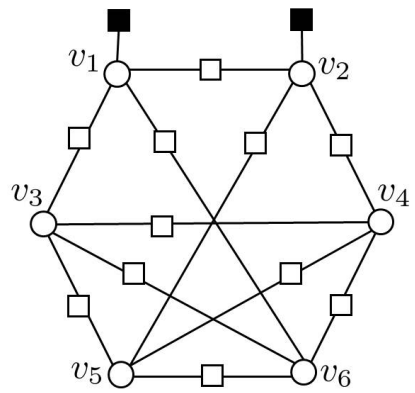

(b)

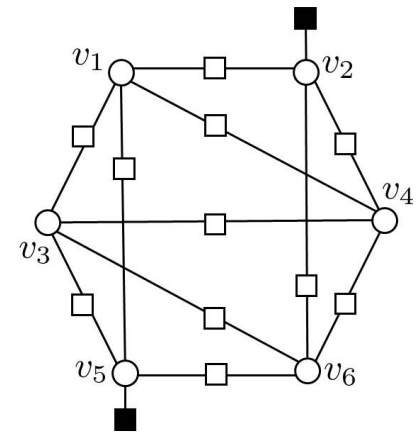

(c)

Figure 8.2: All the possible non-isomorphic topologies for $(6,2)$ ETSs in left-regular LDPC codes with $d_{l}=4$ and $g=6$.

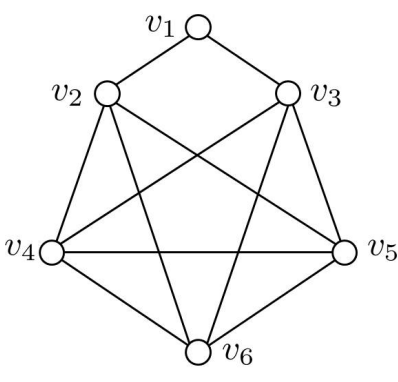

(a)

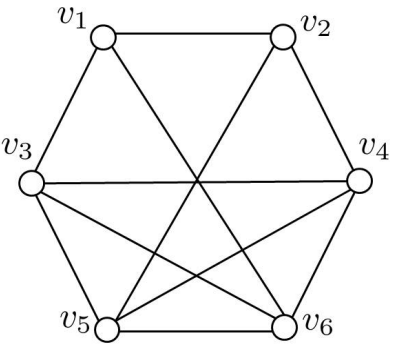

(b)

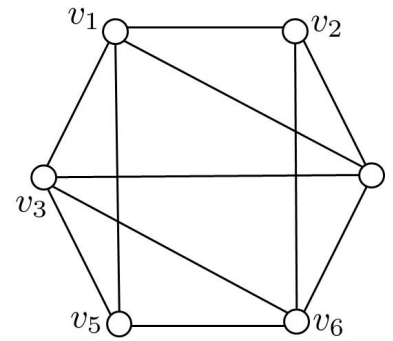

$(c)$

Figure 8.3: All the possible non-isomorphic normal graphs of $(6,2)$ ETSs in leftregular LDPC codes with $d_{l}=4$ and $g=6$.

There are only two possibilities for $(6,2)$ ETSs: (i) Both unsatisfied check nodes are connected to the same variable node, and (ii) the two unsatisfied check nodes are connected to two different variable nodes. We show that there is only one structure for the first case and only 2 non-isomorphic structures for the second case.

For Case (i), let $v_{1}$ be the only variable node connected to two unsatisfied check nodes. This means that in the normal graph representation, $v_{1}$ is connected to only two other variable nodes. Suppose that those variable nodes are $v_{2}$ and $v_{3}$. Since the degree of all the other variable nodes $v_{4}, v_{5}$ and $v_{6}$ in the normal graph is 4 , each of them must have 4 distinct neighbors. The only possibility is for each of these three nodes to be connected to the other two nodes in addition to nodes $v_{2}$ and $v_{3}$. This also satisfies the degree requirements for $v_{2}$ and $v_{3}$ (each of them has degree 4 and is connected to all the nodes $v_{4}, v_{5}, v_{6}$ and $\left.v_{1}\right)$. This topology is shown in Figure 8.3(a). 
For Case (ii), let $v_{1}$ and $v_{2}$ be the two variable nodes, each connected to one unsatisfied check node. Since all the other 4 nodes have degree 4 and must be connected to 4 distinct nodes, each of them must be connected to at least one of the nodes $v_{1}$ and $v_{2}$. There are two possibilities: a) $v_{1}$ and $v_{2}$ are connected together, and $\left.\mathrm{b}\right) v_{1}$ and $v_{2}$ are not connected together. One can show that the only possible topology for Case (a) is the one shown in Figure 8.3(b), and the only possible topology for Case (b) is the one shown in Figure 8.3(c). For Case (a), there always exists a node $v_{3}$ which is connected to one of the nodes $v_{1}$ and $v_{2}$, but not to both. Otherwise, if all the other 4 nodes are connected to both $v_{1}$ and $v_{2}$, both $v_{1}$ and $v_{2}$ will have degree 5 which contradicts the assumption of the proposition that $d_{l}=4$. Without loss of generality, we assume $v_{3}$ to be connected to $v_{1}$, and we grow the normal graph from $v_{3}$ as the root. Figure 8.4(a) shows such a graph growth, in which all the nodes except $v_{2}$ are located in the first layer and $v_{2}$ is in the second layer. Node $v_{2}$ must be connected to two nodes other than $v_{1}$. Without loss of generality, we assume those nodes to be $v_{4}$ and $v_{5}$ (Figure 8.4(b)). From this point on, there is no option in connecting the nodes. Node $v_{6}$ must be connected to 4 distinct nodes, and the only possibility is to have it connected to all the nodes $v_{4}, v_{5}$ and $v_{1}$. This will satisfy the degree of $v_{1}$, which must be 3 . To satisfy the degrees of $v_{4}$ and $v_{5}\left(d\left(v_{4}\right)=d\left(v_{5}\right)=4\right)$, the only option is to have a connection between them, which results in the topology shown in Figure 8.3(b). The proof that Case (b), i.e., the case where $v_{1}$ and $v_{2}$ are not connected, will result in the topology of Figure $8.3(c)$ is similar and is thus omitted.

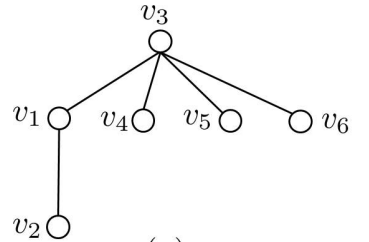

(a)

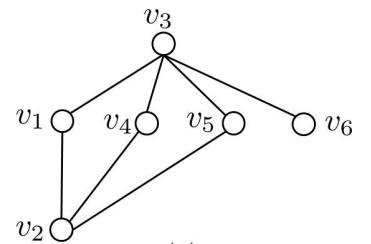

$(b)$

Figure 8.4: Growing the normal graph for Case ii(a) in the proof of Proposition 5.

Remark 11. The structures in Figures 8.2(b) and 8.2(c) are absorbing sets while the one in Figure 8.2(a) is not. Moreover, Proposition 5 implies that there are no $(6,2)$ ETSs in left-regular graphs with $d_{l}=4$ and $g>6$.

Finding the non-isomorphic structures for ETSs with rather large values of $d_{l}$, $a$ or $b$ can be a formidable task. We thus resort to software programs to find such 
structures. One of the well-known software programs related to graph isomorphism is the nauty program [131]. This program can be used to efficiently generate all the non-isomorphic graphs with a given number of nodes (up to 32) and a given number of edges. The program has many input options to determine the minimum and maximum values of the node degrees, to select the girth, and to generate only bipartite graphs or all the possible graphs. For the case of bipartite graphs, however, the program does not have the option of taking the degree distribution of each part of the graph as an input. In the case of finding the non-isomorphic structures (for induced subgraphs) of ETSs, this limitation results in having a large number of undesired structures at the output of the program. One is thus required to check all the output structures to find the ones that satisfy the particular degree distributions of the class of ETSs under consideration. This difficulty can be circumvented by using the normal graph representation of ETSs as explained in the following example.

Example 33. Consider the class of $(6,6)$ ETSs in a left-regular graph with $d_{l}=4$ and $g=6$. Figure 8.5(a) shows one such ETS.

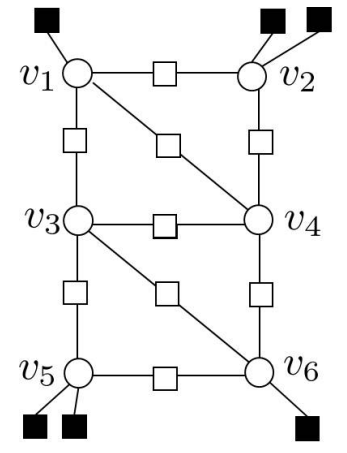

(a)

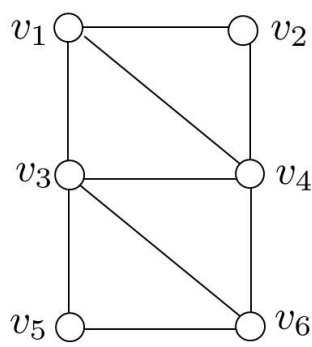

(b)

Figure 8.5: A $(6,6)$ elementary trapping set and its normal graph.

It is easy to see that the induced subgraph of any ETS in this class has 6 variable nodes of degree 4, 6 check nodes of degree 1 , and $((6 \times 4)-6) / 2=9$ check nodes of degree 2. This is a total of 21 nodes (check and variable nodes) and 24 edges. To generate the non-isomorphic structures of $(6,6)$ ETSs using nauty package, one way is to set the package parameters to generate all the connected bipartite graphs of girth at least 6 with 21 nodes and 24 edges, and with the minimum and maximum degrees of 1 and 4, respectively. This results in 53,727,932 graphs, from which an overwhelming 
majority are not $(6,6)$ ETSs.

Alternatively, we can use the normal graph representation of $(6,6)$ ETSs. Figure 8.5(b) shows the normal graph of the structure shown in Figure 8.5(a). Similar to Figure 8.5(b), any normal graph of a $(6,6)$ ETS in a left-regular graph with $d_{l}=4$ has 6 nodes and 9 edges. To generate all the non-isomorphic normal structures using the nauty program, one needs to generate all the bipartite and non-bipartite graphs with 6 nodes and 9 edges, and with the minimum and maximum node degrees of 2 and 4, respectively. This reduces the number of possible graphs from 53, 727, 932 to only 11. All the 11 graphs correspond to valid structures for the class of ETSs under consideration. These structures are shown in Figures 8.6, 8.7, 8.8, and 8.9. Among the 11 structures, only 2 structures are absorbing sets. These are shown in Figure 8.6. The other structures are grouped together based on the number of variable nodes with two unsatisfied check nodes. The structures with 1, 2, and 3 variable nodes connected to two unsatisfied check nodes are shown in Figures 8.7, 8.8, and 8.9, respectively.
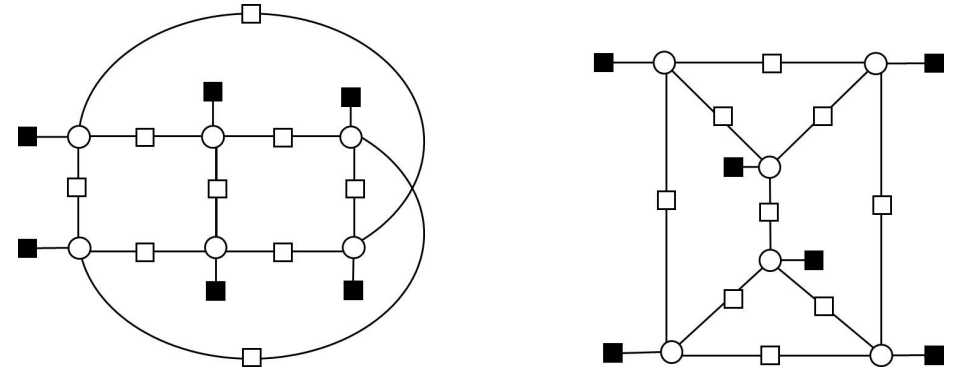

Figure 8.6: Possible topologies for a $(6,6)$ ETS in a left-regular graph with $d_{l}=4$ and $g=6$ : the only two possible absorbing set topologies.
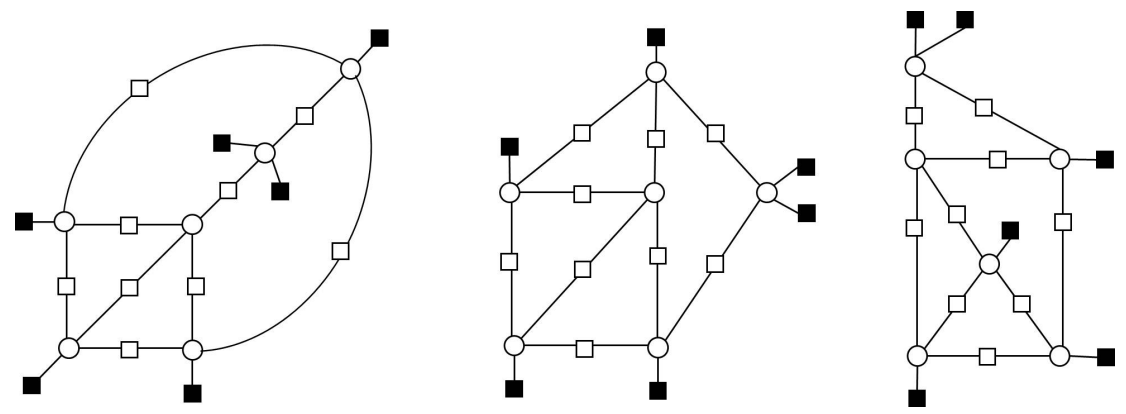

Figure 8.7: Possible topologies for a $(6,6)$ ETS in a left-regular graph with $d_{l}=4$ and $g=6$ : topologies with only one variable node connected to two unsatisfied check nodes. 

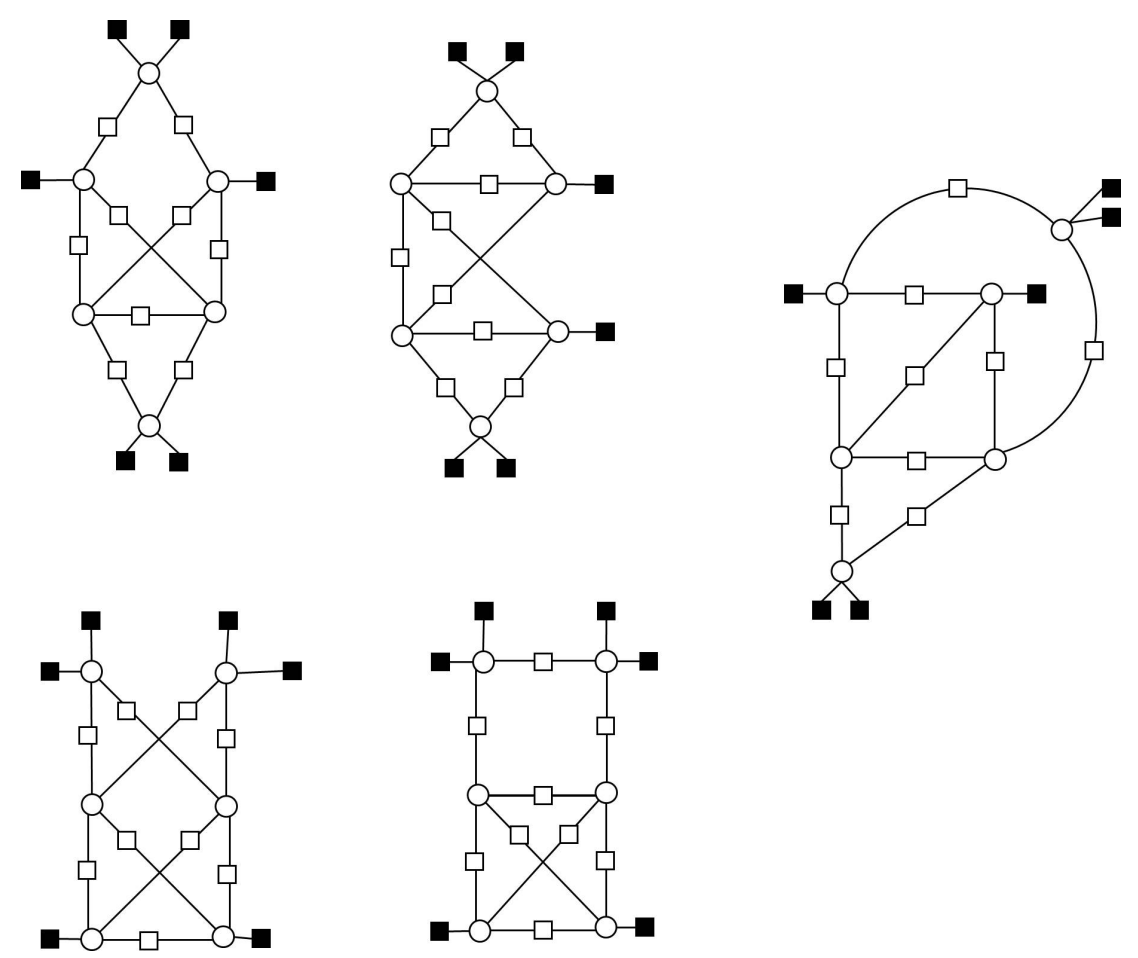

Figure 8.8: Possible topologies for a $(6,6)$ ETS in a left-regular graph with $d_{l}=4$ and $g=6$ : topologies with only two variable nodes connected to two unsatisfied check nodes.

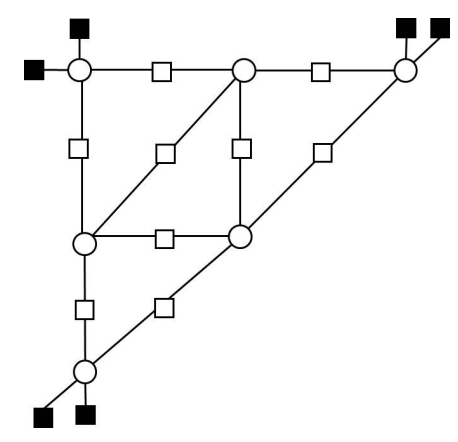

Figure 8.9: Possible topologies for a $(6,6)$ ETS in a left-regular graph with $d_{l}=4$ and $g=6$ : the only possible topology with three variable nodes connected to two unsatisfied check nodes. 


\subsection{Layered Superset (LSS) Property}

Consider an $(a, b)$ ETS $\mathcal{S}$ in $\mathcal{T}$. Let $C \subset \mathcal{S}$ be an ETS in $\mathcal{T}$ of size $\alpha<a$. We say that $\mathcal{S}$ is a layered superset (LSS) of $C$ if there exists a nested sequence of ETSs: $C \triangleq \mathcal{S}^{(0)} \subset \mathcal{S}^{(1)} \subset \cdots \subset \mathcal{S}^{(a-\alpha)} \triangleq \mathcal{S}$, such that $\mathcal{S}^{(i)} \in \mathcal{T}$ has size $\alpha+i$ for $i=0, \ldots, a-\alpha$. When there is no risk of confusion, we also refer to $\mathcal{S}$ as having the LSS property.

Example 34. Consider the ETS $\mathcal{S}=\left\{v_{1}, v_{2}, v_{3}, v_{4}, v_{5}, v_{6}\right\}$ of a left-regular LDPC code with $d_{l}=4$ shown in Figure 8.10(a), and one of its subsets $C_{1}=\left\{v_{1}, v_{2}, v_{3}\right\}$. It is easy to see that $\mathcal{S}$ and $C_{1}$ are $(6,6)$ and $(3,6)$ ETSs. Careful inspection of

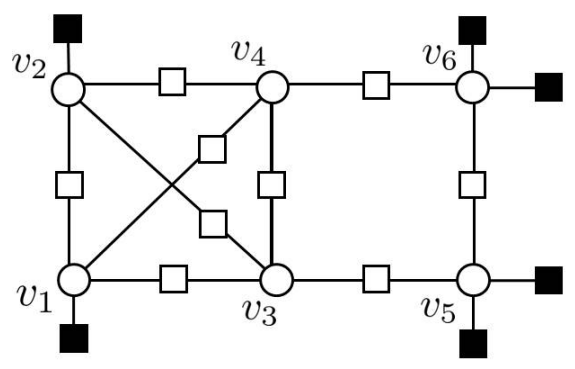

(a)

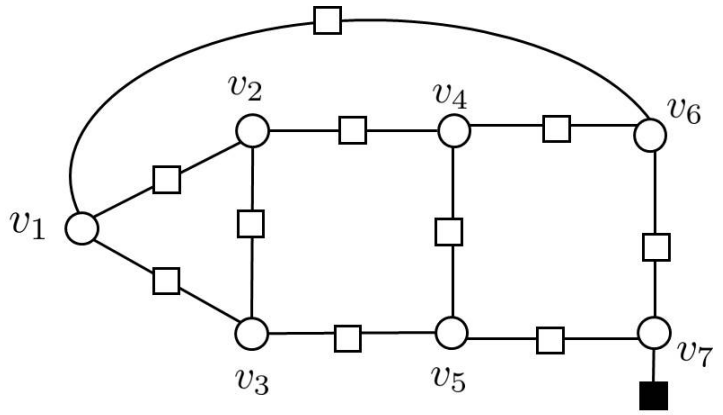

(b)

Figure 8.10: (a) A $(6,6)$ ETS in a left-regular graph with $d_{l}=4$, (b) A $(7,1)$ ETS in a left-regular graph with $d_{l}=3$.

Figure 8.10(a) reveals that $\mathcal{S}$ is not an $L S S$ of $C_{1}$. Set $\mathcal{S}$ however, is an LSS of $C_{2}=$ $\left\{v_{3}, v_{4}, v_{5}, v_{6}\right\}$ with the following nested sequence of ETSs: $C_{2} \subset\left\{v_{1}, v_{3}, v_{4}, v_{5}, v_{6}\right\} \subset$ $\mathcal{S}$. Figure $8.10(b)$ shows a $(7,1)$ ETS $\mathcal{S}$ in a left-regular code with $d_{l}=3$. This set has LSS property with respect to $C=\left\{v_{2}, v_{3}, v_{4}, v_{5}\right\}$ with the following nested sequence of ETSs: $C \subset\left\{v_{1}, v_{2}, v_{3}, v_{4}, v_{5}\right\} \subset\left\{v_{1}, v_{2}, v_{3}, v_{4}, v_{5}, v_{6}\right\} \subset \mathcal{S}$.

One should note that any cycle in a Tanner graph is an ETS. In the sequel, we are particularly interested in the LSS property of more complex ETSs with respect to short cycles of the graph. The following proposition is an example.

Proposition 6. All the $(6,2)$ ETSs of a left-regular LDPC code with $d_{l}=4$ and $g=6$ are layered supersets of any one of their 6-cycle subsets.

Proof. Based on Proposition 5, there are only 3 non-isomorphic structures for $(6,2)$ ETSs in left-regular LDPC codes with $d_{l}=4$ and $g=6$. These structures are 
shown in Figure 8.2. It is not difficult to see that all three structures are LSS of any of their 6-cycle subsets. For example, the structure $\mathcal{S}$ in Figure 8.2(b) is an LSS of its 6-cycle subset $C=\left\{v_{1}, v_{3}, v_{6}\right\}$ with the following nested sequence of ETSs: $C \subset\left\{v_{1}, v_{3}, v_{6}, v_{5}\right\} \subset\left\{v_{1}, v_{3}, v_{6}, v_{5}, v_{4}\right\} \subset \mathcal{S}$.

One advantage of LSS property is that it corresponds to a simple algorithm for finding larger ETSs with LSS property starting from one of their subsets in the nested sequence of ETSs. The basic step is explained in the following lemma, which is basically the same as Lemma 14 .

Lemma 19. Consider an ETS $\mathcal{S} \in \mathcal{T}$ of size $a+1$. Suppose that $\mathcal{S}$ has an elementary trapping subset $\mathcal{S}^{\prime} \in \mathcal{T}$ of size a. Then, the variable node $v \in \mathcal{S} \backslash \mathcal{S}^{\prime}$ is only connected to unsatisfied check nodes of $\mathcal{S}^{\prime}$ (at least two of them), i.e., there is no connection between $v$ and the satisfied check nodes of $\mathcal{S}^{\prime}$.

The pseudo code of an algorithm corresponding to Lemma 19 is given in Routine 1.

\section{Routine 1: Expansion of an ETS $\mathcal{S}$ of size $a$ to ETSs of size $a+1$}

Routine EX = OneExpansion $(\mathcal{S})$

\section{1: Initialization: $\mathrm{EX} \leftarrow \emptyset$.}

2: Let $\Gamma_{\mathrm{o}}(\mathcal{S})$ and $\Gamma_{\mathrm{e}}(\mathcal{S})$ be the set of unsatisfied check nodes and satisfied check nodes of $\mathcal{S}$, respectively.

3: Let $\mathcal{O}_{2}(\mathcal{S})$ be the set of variable nodes which have at least two connections with the check nodes in $\Gamma_{\mathrm{o}}(\mathcal{S})$ and have no connection with the check nodes in $\Gamma_{\mathrm{e}}(\mathcal{S})$.

4: for each element $v$ in $\mathcal{O}_{2}(\mathcal{S})$ do

5: $\quad \mathcal{S}^{\prime} \leftarrow \mathcal{S} \cup v$.

6: $\quad \mathrm{EX} \leftarrow \mathrm{EX} \cup \mathcal{S}^{\prime}$

7: end for

8: Output: EX

Remark 12. Based on Lemma 19, the set $\mathcal{O}_{2}(\mathcal{S})$ contains all the nodes that can be part of the expansion of the ETS. 
Remark 13. Complexity of Routine 1 for expanding an $(a, b)$ ETS to ETSs of size $a+1$ in $a\left(d_{l}, d_{r}\right)$ regular graph, where $d_{l}$ and $d_{r}$ are left and right degrees, respectively, is of order $O\left(b d_{r}\right)$. This comes from the fact that for finding the larger trapping sets, the algorithm needs to check at most $b\left(d_{r}-1\right)$ variable nodes as possible candidates. The memory requirement in this case is of order $O\left(a b d_{r}\right)$. It should be however noted that, imposing the condition of Line 3 removes a large portion of neighboring variable nodes of $\mathcal{S}$ from the set of possible candidates for expansion. This highly reduces the complexity of the algorithm.

Consider the case where an ETS $\mathcal{S}$ of size $a$ is an LSS of an ETS $C$ of size $\alpha$. Clearly, starting from $C$, the successive application of Routine 1 will result in finding all the ETSs which are layered supersets of $C$. In particular, ETS $\mathcal{S}$ will be among the outputs after $a-\alpha$ applications of Routine 1. Algorithm 5 contains the pseudo code of an algorithm that starts from a set of ETSs and finds all the ETSs of size up to $k$ that are layered supersets of the initial set of ETSs.

Algorithm 5: Expansion of input ETSs to ETSs of size up to $k$ in $G=(L \cup R, E)$. $\left(\mathcal{L}_{\text {in }}\right.$ and $\mathcal{L}_{\text {out }}$ are the lists of input and output trapping sets, respectively.)

1: Inputs: $G, \mathcal{L}_{\text {in }}, k$.

2: Initialization: $\mathcal{L}_{\text {out }}^{i}=\left\{\mathcal{S} \in \mathcal{L}_{\text {in }},|\mathcal{S}|=i\right\}$ for $i=2, \ldots, k$.

3: for each $i<k$ starting from the smallest one for which $\mathcal{L}_{\text {out }}^{i} \neq \emptyset$ do

4: $\quad$ for each element $\mathcal{S} \in \mathcal{L}_{\text {out }}^{i}$ do

5: $\quad \mathrm{EX}=$ OneExpansion $(\mathcal{S})$

6: $\quad \mathcal{L}_{\text {out }}^{i+1} \leftarrow \mathcal{L}_{\text {out }}^{i+1} \cup$ EX.

7: $\quad$ end for

8: end for

9: Output: $\mathcal{L}_{\text {out }}=\mathcal{L}_{\text {out }}^{2} \cup \cdots \cup \mathcal{L}_{\text {out }}^{k}$. 


\subsection{Characterization of Elementary Trapping Sets of Left-Regular LDPC Codes}

\subsubsection{Motivating Examples}

It is easy to see that any trapping set in $\mathcal{T}$, including ETSs, contains at least one cycle. It can thus be argued that cycles are the most basic structure for ETSs in $\mathcal{T}$. This motivates the study of the relationship between cycles, as the most basic ETSs, and the more complex ETSs of $\mathcal{T}$. Short cycles have long been known to be problematic for iterative decoding in general [72], and for the performance in the error floor region, in particular [121], [122]. Short cycles are also easy to enumerate [121]. In this section, we establish a simple relationship between short cycles and the more complex ETSs. In particular, we prove that an overwhelming majority of dominant ETSs of left-regular LDPC codes are layered supersets of short cycles. One important implication of this result is that, starting from short cycles of the graph, Algorithm 5, presented in the previous section, can be used to find all such ETSs in a guaranteed fashion.

The following examples demonstrate the relationship between short cycles and the more complex ETSs of left-regular graphs.

Example 35. Figure 8.11 shows a possible structure $\mathcal{S}$ of a $(5,1)$ ETS in a leftregular graph with $d_{l}=3$ and $g=6$. The $(3,3)$ ETS $C=\left\{v_{1}, v_{2}, v_{3}\right\}$ forms a 6 -cycle and is a subset of $\mathcal{S}$. Set $\mathcal{S}$ is an LSS of C with the following nested sequence of ETSs: $C \subset \mathcal{S}^{(1)}=\left\{v_{1}, v_{2}, v_{3}, v_{4}\right\} \subset \mathcal{S}$. Starting from $C$, in the first round of expansion by Algorithm 5, variable node $v_{4}$ will be added and the $(4,2)$ ETS $\mathcal{S}^{(1)}$ will be found. In the second round of expansion, with set $\mathcal{S}^{(1)}$ as the input, variable node $v_{5}$ will be added and $\mathcal{S}$ will be found.

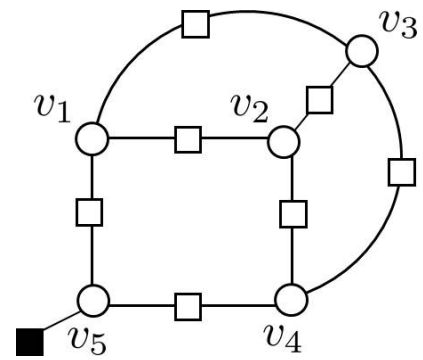

Figure 8.11: A $(5,1)$ trapping set in a left-regular graph with $d_{l}=3$. 
Note that although $v_{5}$ is not part of any 6-cycle, this node will be added at the second round of expansion. In other words, for a trapping set to be found by the algorithm, it is not necessary that all the nodes in the set participate in short(est) cycles.

Starting from a short cycle, each round of expansion by Algorithm 5, may result in several new ETSs. These trapping sets all have the same size, but may have different number of unsatisfied check nodes, and thus belong to different classes of trapping sets. This is demonstrated in the following example.

Example 36. Consider the structure in Figure 8.2(b) and one of its subsets $C=$ $\left\{v_{3}, v_{4}, v_{6}\right\}$ which is a $(3,6)$ ETS. Starting from C, as the input to Algorithm 5, there are two variable nodes $v_{1}$ and $v_{5}$ as candidates for the first expansion. The resultant ETSs are $\left\{v_{3}, v_{4}, v_{6}, v_{1}\right\}$ and $\left\{v_{3}, v_{4}, v_{6}, v_{5}\right\}$, which are $(4,6)$ and $(4,4)$ trapping sets, respectively.

It is important to note that if an element of a class of ETSs is an LSS of a short cycle, this does not necessarily mean that all the elements of that class are also LSSs of short cycles of the same length. The reason is that there may be other non-isomorphic structures in that class which are not LSSs of any of the short cycles under consideration.

Example 37. Figure 8.12 shows two possible structures for a $(6,6)$ ETS in a leftregular graph with $d_{l}=4$ and $g=6$. The structure of Figure 8.12(a) is an LSS of any of its 6-cycles and thus can be obtained by the recursive expansion of any of them through Algorithm 5. The structure of Figure 8.12(b), on the other hand, is not an LSS of any of its 6-cycles, and is thus out of the reach of Algorithm 5, if the algorithm starts from any 6-cycle.

It is however, easy to see that the structure in Figure 8.12(b) is an LSS of the set $\left\{v_{3}, v_{4}, v_{5}, v_{6}\right\}$, which itself forms an 8-cycle. The trapping set can thus be found by Algorithm 5 if cycles of length 8 are included in the input set. In general, adding short cycles longer than the girth to the input of Algorithm 5 can improve the coverage of this algorithm. Nonetheless, there are structures which do not satisfy the LSS property with respect to any of their cycles. This means that even arbitrarily enlarging the size of the cycles in the initial input set will not result in finding such structures by the algorithm. 


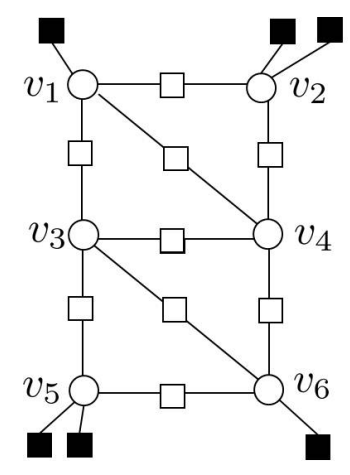

$(a)$

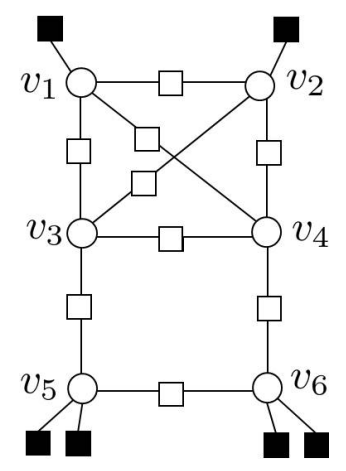

(b)

Figure 8.12: Two possible structures of a $(6,6)$ ETS in a left-regular graph with $d_{l}=4$ and $g=6$.

Example 38. Figure 8.13 shows an $(8,2)$ ETS $\mathcal{S}$ in a left-regular graph with $d_{l}=3$ and $g=6$. Set $\mathcal{S}$ contains cycles of length 6, 8 and 10. It is easy to check that $\mathcal{S}$ does not satisfy the LSS property with respect to any of these cycles, and thus cannot be found using Algorithm 5 starting from any of them.

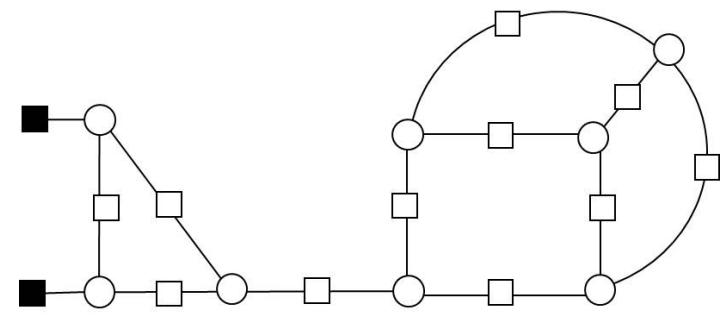

Figure 8.13: An $(8,2)$ ETS in a left-regular graph with $d_{l}=3$ and $g=6$ which is not an LSS of any of its cycles.

\subsubsection{Non-Isomorphic Structures of Dominant ETSs and Their Characterization}

In this part of the chapter, we investigate the structure of ETSs of left-regular LDPC codes with left degrees $3,4,5$ and 6 . For each category of codes, we consider girth values 6 and 8 , and study all the non-isomorphic structures of different classes of $(a, b)$ ETSs with values of $a$ and $b$ up to 10. For each class of ETSs with given values of $a$ and $b$, we first find all the non-isomorphic structures using the approach described in 
Section III (with constraints on $d_{l}$ and $g$ ). We then examine each of these structures to find out whether the structure is an LSS of any of its cycles. This can be performed using Algorithm 5. Let $\mathcal{S}$ be the structure under consideration. We can start with the set of shortest cycles, say of length $\ell$, in $\mathcal{S}$, and apply Algorithm 5 to recursively expand them to larger subsets of $\mathcal{S}$. If this process ends with finding $\mathcal{S}$, then we report $\mathcal{S}$ as being an LSS of a cycle of length $\ell$. In the case that this process will not result in finding $\mathcal{S}$, we use the set of cycles of next larger size as the input and repeat the process. This will continue until $\mathcal{S}$ is identified as an LSS of one of its cycles or until all the cycles are exhausted and $\mathcal{S}$ is not an LSS of any of them. In the former case, if the cycle length is $x$, we refer to $\mathcal{S}$ as an $\operatorname{LSS}_{x}$ structure. The results for different values of $d_{l}$ and $g$ are reported in the following subsections. For each value of $a$, we mostly consider the values of $b$ which satisfy $b / a \leq 1$. For a given value of $a$, these values of $b$ are believed to correspond to dominant trapping sets [90]. It is easy to see that for a Tanner graph with girth $g=8$, it is impossible to have an $(a, b)$ trapping set in $\mathcal{T}$ with $a<4$. For a Tanner graph with $g=6$, there are no trapping sets in $\mathcal{T}$ with $a<3$. For such a Tanner graph, the only possible structure for ETSs with $a=3$ is a 6 -cycle, which forms a $\left(3,3\left(d_{l}-2\right)\right)$ ETS. Thus, in the following, we only consider $(a, b)$ trapping sets with $a \geq 4$.

The Case $d_{l}=3, g=6$

Theorem 7. For left-regular graphs with $d_{l}=3$ and $g=6$, the multiplicity of nonisomorphic $L S S_{x}$ structures for different values of $x$ are listed in Table 8.1 for different classes of ETSs in $\mathcal{T}$.

(Each row in Table 8.1 corresponds to a specific size $a$ of an ETS, and each column corresponds to a specific number of unsatisfied check nodes, $b$. For each pair $(a, b)$, Table 8.1 lists the multiplicity of $\operatorname{LSS}_{x}$ structures for different values of $x$. For example, having $\left\{\begin{array}{cc}g+2 & g+4 \\ 3 & 1\end{array}\right\}$ for a specific class of ETSs means that there are 4 different non-isomorphic structures for that class of trapping sets: Three of them are $\mathrm{LSS}_{g+2}$ and one of them is an $\mathrm{LSS}_{g+4}$ structure. Starting with cycles of length $g+2$ and $g+4$, Algorithm 5 is thus guaranteed to find all such ETSs. Having the symbol "-" for a class of $(a, b)$ trapping sets means that for the underlying conditions, i.e., $d_{l}=3$ and $g=6$, it is impossible to have such a class of trapping sets. For the structures which do not satisfy the LSS property with respect to any of their cycles, 
Table 8.1: LSS Properties of Non-isomorphic Structures of $(a, b)$ ETS Classes for Leftregular Graphs with $d_{l}=3$ and $g=6$.

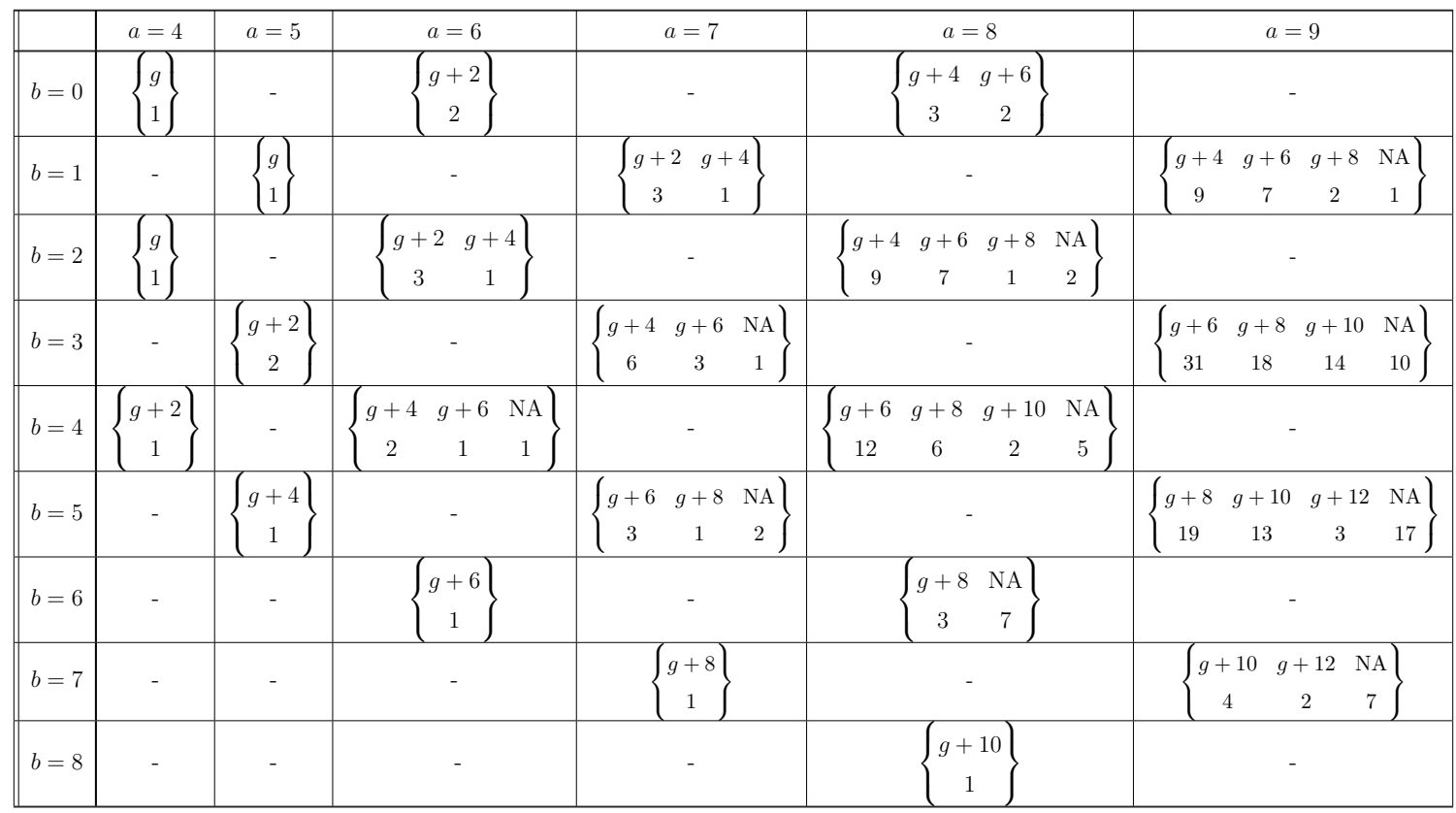

we use the notation "NA" (stands for not applicable). Starting from any set of cycles of the graph, Algorithm 5 cannot find such structures.)

Proof. In general, the results reported in Table 8.1 for any $(a, b)$ ETS can be proved by first obtaining all the non-isomorphic structures using the nauty program, as described in Section III, and then examining each such structure for the LSS property, using Algorithm 5, as described earlier in this subsection. We however, provide a formal proof for the results pertaining to the ETS class $(6,0)$ as well as all the classes that cannot exist in left-regular graphs with $d_{l}=3$ and $g=6$ (i.e., those with designation "-" in the table). The general approach for the formal proof of the rest of the results is similar and not provided.

$(6,0)$ ETSs: We first prove that the two structures presented in Figure 8.14 are the only possible non-isomorphic structures for $(6,0)$ ETSs in left-regular graphs with $d_{l}=3$ and $g=6$. We use the normal graph representation of these structures, also shown in Figure 8.14, for the proof.

Since the number of unsatisfied check nodes is zero, every node in the normal graph must be connected to three other nodes. Starting from an arbitrary node, say $v_{3}$, as the root, we grow the normal graph, and will have three nodes in the first layer. 

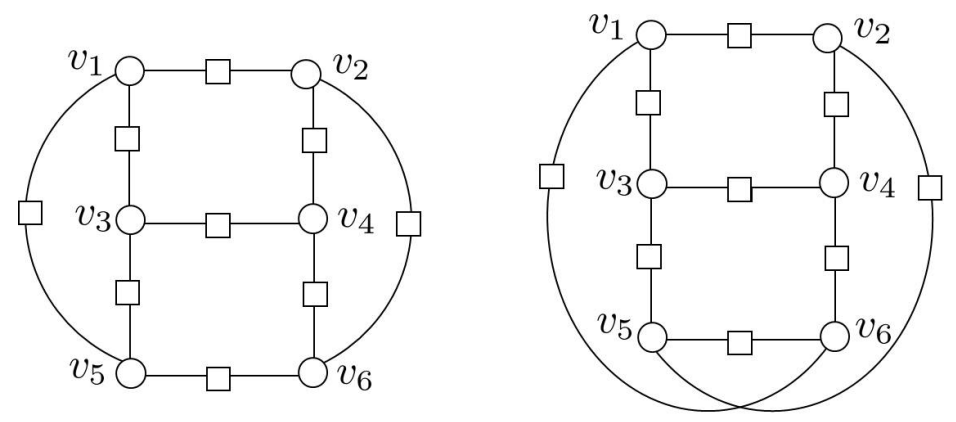

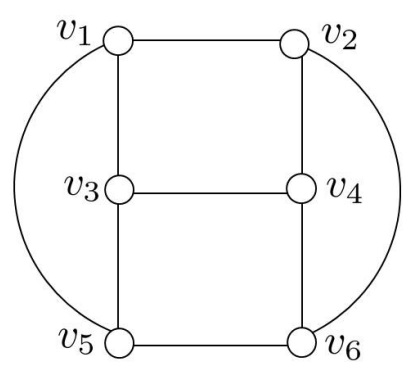

(a)

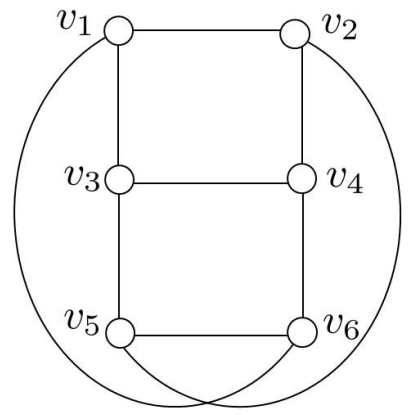

(b)

Figure 8.14: The only possible non-isomorphic structures for $(6,0)$ ETSs in leftregular graphs with $d_{l}=3$ and $g=6$, and their normal graph representations.

We arbitrarily denote these nodes by $v_{1}, v_{4}$, and $v_{5}$. Consider the case where the nodes in the first layer do not have any edges in common (see Figure 8.15(a)). In this case, they must have 6 edges connected to the two remaining nodes $\left(v_{2}\right.$ and $\left.v_{6}\right)$. According to the girth constraint (i.e., $g=6$ ), each pair of nodes can have at most one edge in common. This implies that the only possible scenario is the case where each of the three nodes in the first layer is connected to both $v_{2}$ and $v_{6}$. This results in the structure of Figure 8.14(b). Note that in this case, the length of the shortest cycles in the structure is 8 .

Now, consider the case where the nodes in the first layer have some edge(s) in common. Based on the girth constraint $(g=6)$, the two remaining nodes $\left(v_{2}\right.$ and $\left.v_{6}\right)$ must have at least 4 edges in common with the nodes in the first layer. (Otherwise, for $v_{2}$ and $v_{6}$ to have degree 3 , they need to have more than one edge in common, which contradicts the girth constraint.) This implies that the nodes in the first layer can have only one edge in common. Without loss of generality, we assume $v_{1}$ and $v_{4}$ are connected (see Figure 8.15(b)). From this point on, there is no choice in connecting the nodes. Node $v_{5}$ must be connected to both $v_{2}$ and $v_{6}$, and each of the nodes $v_{1}$ 
and $v_{4}$ must have one connection to $v_{2}$ or $v_{6}$. (Switching the connections will result in isomorphic structures.) This results in the structure of Figure 8.14(a).

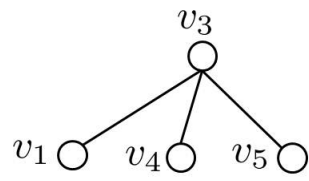

$v_{2} \bigcirc \quad \bigcirc v_{6}$

(a)

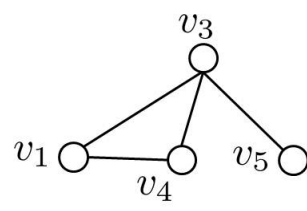

$v_{2} \bigcirc \quad \bigcirc v_{6}$

Figure 8.15: Growing the normal graph of $(6,0)$ ETSs of a left-regular graph with $d_{l}=3$ and $g=6$.

There are two cycles of length 6 in the structure $\mathcal{S}_{1}$ of Figure 8.14(a). However, $\mathcal{S}_{1}$ is not an LSS of any of them. There are also three cycles of length 8 in $\mathcal{S}_{1}$, and $\mathcal{S}_{1}$ is an LSS of all of them. For example, $\mathcal{S}_{1}$ is an LSS of $C_{1}=\left\{v_{1}, v_{3}, v_{4}, v_{2}\right\}$ with the following nested sequence of ETSs: $C_{1} \subset\left\{v_{1}, v_{3}, v_{4}, v_{2}, v_{5}\right\} \subset \mathcal{S}_{1}$. The structure $\mathcal{S}_{2}$ of Figure 8.14(b) does not have any 6-cycles. It has four 8-cycles and has LSS property with respect to all of them.

Non-existent structures: All such cases in Table 8.1 can be proved using two facts: (i) An $(a, b)$ ETS in a left-regular graph with $d_{l}=3$ has $3 a$ edges and $(3 a-b) / 2$ satisfied check nodes. For the latter to be integer, there cannot be any $(a, b)$ ETS with an odd $a$ and an even $b$, or vice versa. (ii) By definition, each variable node of an ETS $\mathcal{S} \in \mathcal{T}$ of a left-regular graph with $d_{l}=3$ can be connected to at most one unsatisfied check node. This means that it is not possible to have an $(a, b)$ ETS with $b>a$.

The results of Table 8.1 indicate that the majority of ETS structures satisfy the LSS property with respect to short cycles. This is particularly the case for smaller values of $a$ and $b$ which correspond to more problematic trapping sets.

Example 39. A cycle of length $2 a$ in a left-regular graph with $d_{l}=3$ is an $(a, a)$ ETS. For example, Figure 8.16 shows the structure of a $(5,5)$ ETS in a left-regular graph with $d_{l}=3$, which is a cycle of length 10. In fact, as the results of Table 8.1 show, cycles of length $2 a$ are the only $(a, a)$ ETSs for the graphs under consideration. Such cycles trivially satisfy the LSS property. 


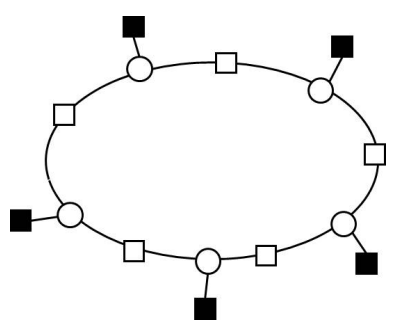

Figure 8.16: A cycle of length 10 is the only possible structure for a $(5,5)$ ETS in a left-regular graph with $d_{l}=3$ and $g=6$.

Example 40. In Figure 8.17, the three structures corresponding to the "NA" cases in Table 8.1 for $(6,4),(7,3)$ and $(9,1)$ ETSs are presented. All three structures consist of two ETSs connected by a check node.

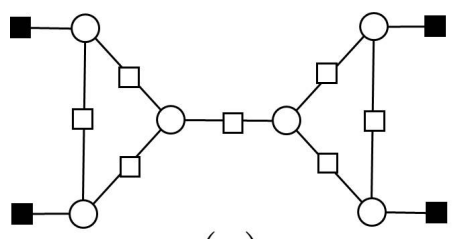

(a)

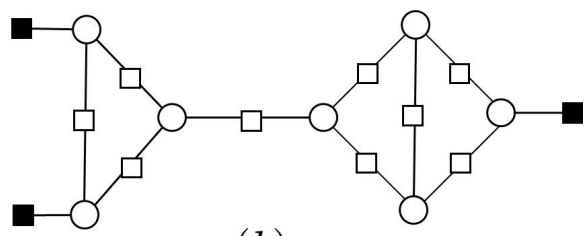

(b)

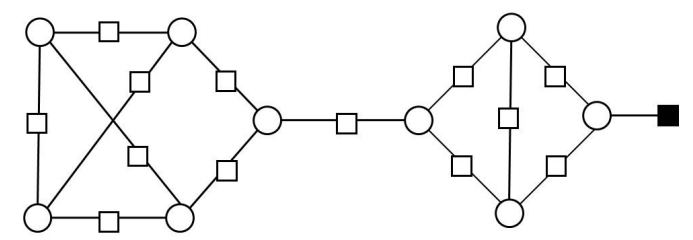

(c)

Figure 8.17: The three structures corresponding to the "NA" cases in Table 8.1 for $(6,4),(7,3)$ and $(9,1)$ ETSs.

In the rest of the chapter, we omit the proofs as they are similar in nature to that of Theorem 7 .

The Case $d_{l}=3, g=8$

Theorem 8. For left-regular graphs with $d_{l}=3$ and $g=8$, the multiplicity of nonisomorphic $L S S_{x}$ structures for different values of $x$ are listed in Table 8.2 for different classes of ETSs in $\mathcal{T}$. 
Table 8.2: LSS Properties of Non-isomorphic Structures of $(a, b)$ ETS Classes for Left-regular Graphs with $d_{l}=3$ and $g=8$.

\begin{tabular}{|c|c|c|c|c|c|c|}
\hline & $a=4$ & $a=5$ & $a=6$ & $a=7$ & $a=8$ & $a=9$ \\
\hline$b=0$ & - & - & $\left\{\begin{array}{l}g \\
1\end{array}\right\}$ & - & $\left\{\begin{array}{cc}g+2 & g+4 \\
1 & 1\end{array}\right\}$ & - \\
\hline$b=1$ & - & - & - & $\left\{\begin{array}{l}g \\
1\end{array}\right\}$ & - & $\left\{\begin{array}{cc}g+2 & g+4 \\
3 & 1\end{array}\right\}$ \\
\hline$b=2$ & - & - & $\left\{\begin{array}{l}g \\
1\end{array}\right\}$ & - & $\left\{\begin{array}{cc}g+2 & g+4 \\
3 & 2\end{array}\right\}$ & - \\
\hline$b=3$ & - & $\left\{\begin{array}{l}g \\
1\end{array}\right\}$ & - & $\mid \begin{array}{cc}g+2 & g+4 \\
2 & 1\end{array}$ & - & $\left\{\begin{array}{cc}g+4 & g+6 \\
13 & 4\end{array}\right\}$ \\
\hline$b=4$ & $\left\{\begin{array}{l}g \\
1\end{array}\right\}$ & - & $\left\{\begin{array}{cc}g+2 & g+4 \\
1 & 1\end{array}\right.$ & S & $\left\{\begin{array}{ccc}g+4 & g+6 & g+8 \\
6 & 2 & 2\end{array}\right\}$ & ( \\
\hline$b=5$ & - & $\left(\begin{array}{c}g+2 \\
1\end{array}\right)$ & - & $\left(\begin{array}{cc}g+4 & g+6 \\
2 & 1\end{array}\right.$ & - & $\left\{\begin{array}{cccc}g+6 & g+8 & g+10 & \mathrm{NA} \\
10 & 7 & 3 & 1\end{array}\right.$ \\
\hline$b=6$ & - & - & $\left\{\begin{array}{c}g+4 \\
1\end{array}\right\}$ & - & $\left\{\begin{array}{ccc}g+6 & g+8 & \mathrm{NA} \\
2 & 2 & 2\end{array}\right\}$ & - \\
\hline$b=7$ & - & - & - & $\left\{\begin{array}{c}g+6 \\
1\end{array}\right\}$ & - & $\left\{\begin{array}{ccc}g+8 & g+10 & \mathrm{NA} \\
3 & 2 & 3\end{array}\right\}$ \\
\hline$b=8$ & - & - & - & - & $\left\{\begin{array}{c}g+8 \\
1\end{array}\right\}$ & - \\
\hline
\end{tabular}

The results of Table 8.2 also indicate that the majority of ETS structures satisfy the LSS property with respect to short cycles of the graph. Compared to the results of Table 8.1, a larger portion of ETS classes have the property that all their nonisomorphic structures are LSSs of short cycles.

Remark 14. In a left-regular graph with $d_{l}=3$, any ETS in $\mathcal{T}$ is also an absorbing set. So the results presented in Tables 8.1 and 8.2 are also applicable to the absorbing sets of these graphs.

The Case $d_{l}=4, g=6$

Theorem 9. For left-regular graphs with $d_{l}=4$ and $g=6$, the multiplicity of nonisomorphic $L S S_{x}$ structures for different values of $x$ are listed in Tables 8.3 and 8.4 for different classes of ETSs in $\mathcal{T}$. 
Table 8.3: LSS Properties of Non-isomorphic Structures of $(a, b)$ ETS Classes for Left-regular Graphs with $d_{l}=4$ and $g=6$.

\begin{tabular}{|c|c|c|c|c|}
\hline & $a=4$ & $a=5$ & $a=6$ & $a=7$ \\
\hline$b=0$ & - & 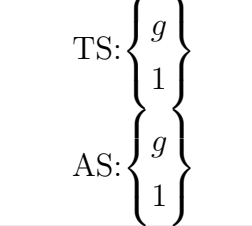 & $\begin{array}{l}\text { TS: }\left\{\begin{array}{l}g \\
1\end{array}\right\} \\
\text { AS: }\left\{\begin{array}{l}g \\
1\end{array}\right\}\end{array}$ & $\begin{array}{l}\left.\text { TS: } \begin{array}{l}g \\
2\end{array}\right\} \\
\text { AS: }\left\{\begin{array}{l}g \\
2\end{array}\right\}\end{array}$ \\
\hline$b=1$ & - & & - & - \\
\hline$b=2$ & - & TS: $\left\{\begin{array}{l}g \\
1 \\
g \\
y \\
1\end{array}\right\}$ & $\begin{array}{l}\left.\text { TS: }: \begin{array}{l}g \\
3\end{array}\right\} \\
\text { AS: }\left\{\begin{array}{l}g \\
2\end{array}\right\}\end{array}$ & $\begin{array}{l}\left.\text { TS: } \begin{array}{l}g \\
9\end{array}\right\} \\
\text { AS: }\left\{\begin{array}{l}g \\
7\end{array}\right\}\end{array}$ \\
\hline$b=3$ & - & & - & - \\
\hline$b=4$ & 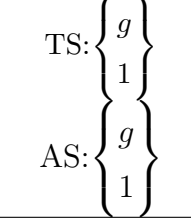 & 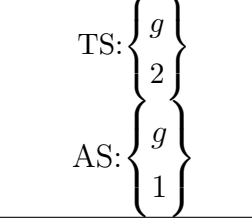 & 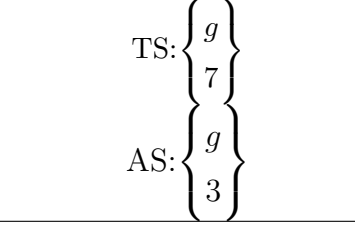 & $\begin{array}{c}\text { TS: }\left\{\begin{array}{ccc}g & g+2 & g+4 \\
25 & 2 & 1\end{array}\right\} \\
\text { AS: }\left\{\begin{array}{cc}g & g+2 \\
9 & 2\end{array}\right\}\end{array}$ \\
\hline$b=5$ & - & & - & - \\
\hline$b=6$ & $\begin{array}{c}\text { TS: }\left\{\begin{array}{l}g \\
1\end{array}\right\} \\
\text { AS: - }\end{array}$ & TS: $\left\{\begin{array}{l}g \\
3\end{array}\right\}$ & $\begin{array}{l}\text { TS: }\left\{\begin{array}{cc}g & g+2 \\
8 & 3\end{array}\right\} \\
\mathrm{AS}:\left\{\begin{array}{c}g+2 \\
2\end{array}\right\}\end{array}$ & $\begin{array}{c}\left.\text { TS }: \begin{array}{cccc}g & g+2 & g+4 & \mathrm{NA} \\
28 & 12 & 3 & 1\end{array}\right\} \\
\mathrm{AS}:\left\{\begin{array}{cc}g+2 & g+4 \\
3 & 1\end{array}\right\}\end{array}$ \\
\hline$b=7$ & - & - & - & - \\
\hline$b=8$ & $\begin{array}{c}\text { TS: }\left\{\begin{array}{c}g+2 \\
1\end{array}\right\} \\
\text { AS: - }\end{array}$ & TS: $\left\{\begin{array}{cc}g+2 & \text { NA } \\
2 & 1\end{array}\right\}$ & $\mathrm{TS}:\left\{\begin{array}{ccc}g+2 & g+4 & \mathrm{NA} \\
8 & 1 & 1\end{array}\right\}$ & TS: $\left\{\begin{array}{cccc}g+2 & g+4 & g+6 & \text { NA } \\
29 & 9 & 1 & 5\end{array}\right\}$ \\
\hline
\end{tabular}

For graphs with $d_{l}>3$, not every ETS is an absorbing set. To identify the structures that are absorbing sets, we use the notation "AS" in the tables. For each class of ETS, we thus have two sets of entries: the ones for absorbing sets denoted by "AS", and those that correspond to all the ETSs denoted by "TS" to stand for "trapping sets". For each entry, the number of possible non-absorbing set structures can be obtained by subtracting the corresponding results in the two sets. For example, the results for the ETS class $(5,4)$ in Table 8.3 indicate that there are two non-isomorphic structures for this class that are both LSSs of cycles of length $g$. Only one of the two structures however, is an absorbing set. 
Table 8.4: LSS Properties of Non-isomorphic Structures of $(a, b)$ ETS Classes for Left-regular Graphs with $d_{l}=4$ and $g=6$.

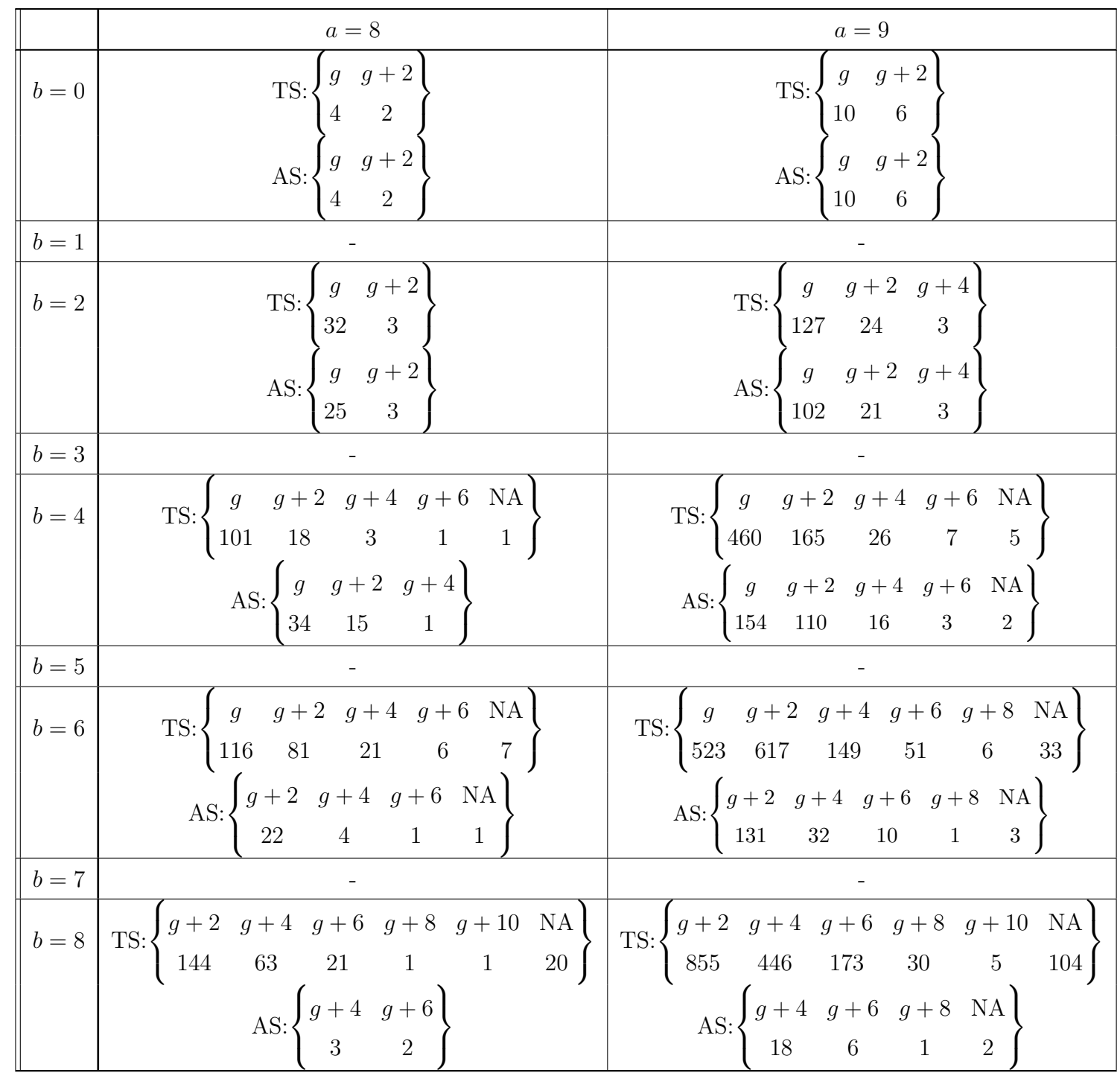

Tables 8.3 and 8.4 indicate that an overwhelming majority of ETS structures satisfy the LSS property with respect to short cycles. In particular, all the ETSs of size less than 7 with less than 6 unsatisfied check nodes are LSSs of 6-cycles.

The Case $d_{l}=4, g=8$

Theorem 10. For left-regular graphs with $d_{l}=4$ and $g=8$, the multiplicity of non-isomorphic $L S S_{x}$ structures for different values of $x$ are listed in Table 8.5 for different classes of ETSs in $\mathcal{T}$. 
Table 8.5: LSS Properties of Non-isomorphic Structures of $(a, b)$ ETS Classes for Left-regular Graphs with $d_{l}=4$ and $g=8$.

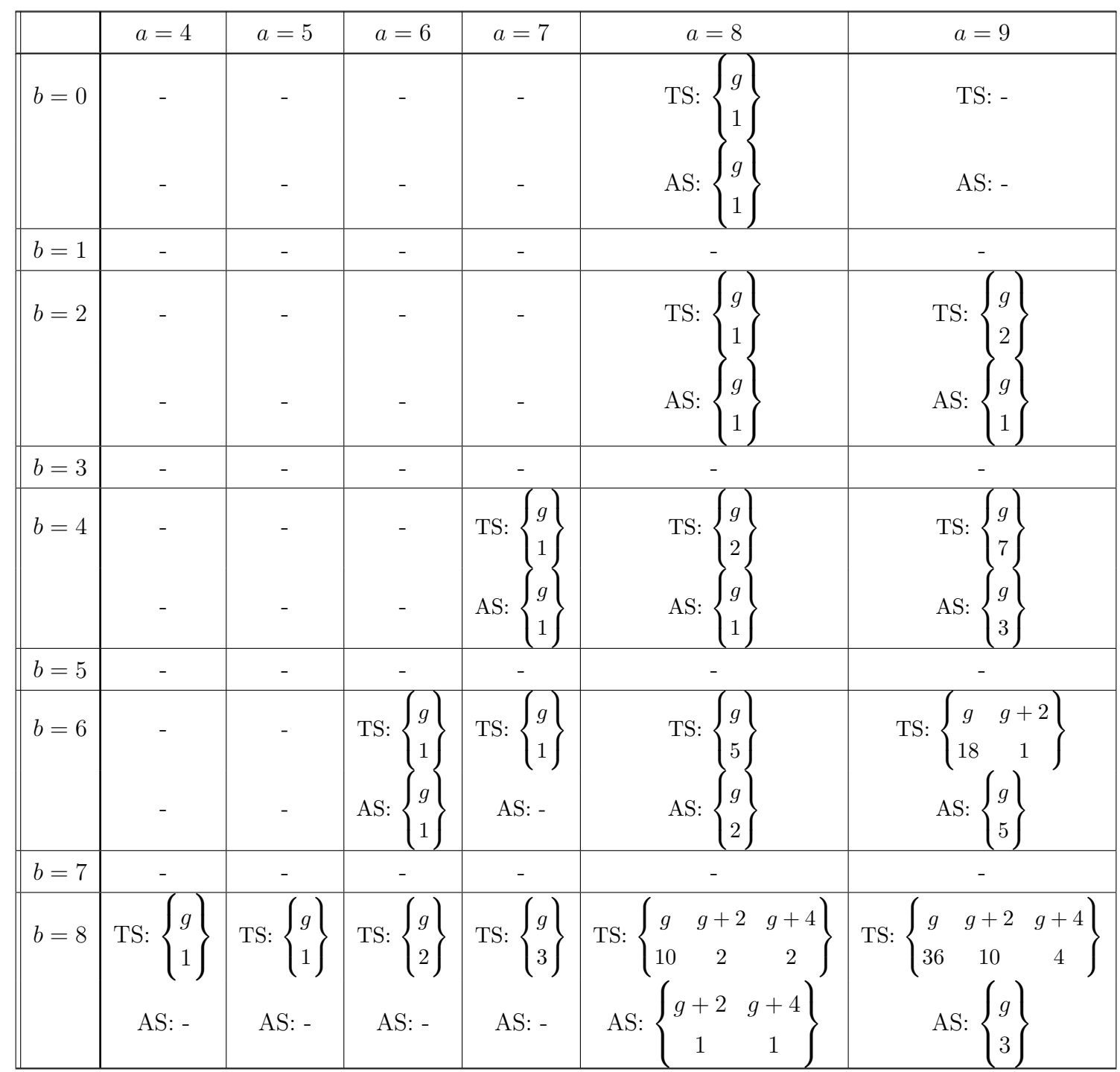

The results of Table 8.5 indicate that all the ETSs of size less than 10 with less than 9 unsatisfied check nodes satisfy the LSS property. Comparison with the results of Tables 8.3 and 8.4 reveals that by increasing the girth of the graph from 6 to 8 , the number of classes whose structures all satisfy the LSS property increases.

The Case $d_{l}=5, g=6$

Theorem 11. For left-regular graphs with $d_{l}=5$ and $g=6$, the multiplicity of non-isomorphic $L S S_{x}$ structures for different values of $x$ are listed in Table 8.6 for 
Table 8.6: LSS Properties of Non-isomorphic Structures of $(a, b)$ ETS Classes for Left-regular Graphs with $d_{l}=5$ and $g=6$.

\begin{tabular}{|c|c|c|c|c|c|c|}
\hline & $a=4$ & $a=5$ & $a=6$ & $a=7$ & $a=8$ & $a=9$ \\
\hline$b=0$ & - & - & TS: $\left\{\begin{array}{l}g \\
1 \\
g \\
1\end{array}\right\}$ & - & TS: $\left\{\begin{array}{l}g \\
3 \\
g \\
3\end{array}\right\}$ & - \\
\hline$b=1$ & - & - & - & TS: $\left\{\begin{array}{l}g \\
1 \\
g \\
1 \\
1\end{array}\right\}$ & $\begin{array}{l}- \\
-\end{array}$ & TS: $\left\{\begin{array}{c}g \\
28 \\
g \\
28\end{array}\right\}$ \\
\hline$b=2$ & - & - & TS: $\left\{\begin{array}{l}g \\
1 \\
g \\
1\end{array}\right\}$ & - & TS: $\left\{\begin{array}{c}g \\
16 \\
g \\
16\end{array}\right\}$ & $\begin{array}{l}- \\
-\end{array}$ \\
\hline$b=3$ & - & - & - & TS: $\left\{\begin{array}{l}g \\
6 \\
g \\
5\end{array}\right\}$ & - & TS: $\left\{\begin{array}{c}g \\
289 \\
g \\
\text { AS: } \\
276\end{array}\right\}$ \\
\hline$b=4$ & - & - & TS: $\left\{\begin{array}{l}g \\
2 \\
g \\
2\end{array}\right\}$ & - & TS: $\left\{\begin{array}{c}g \\
75 \\
g \\
68\end{array}\right\}$ & $\begin{array}{l}- \\
-\end{array}$ \\
\hline$b=5$ & - & AS: $\left\{\begin{array}{l}g \\
1 \\
g \\
1\end{array}\right\}$ & - & $\begin{array}{l}\text { TS: }\left\{\begin{array}{c}g \\
18\end{array}\right\} \\
\text { AS: }\left\{\begin{array}{c}g \\
14\end{array}\right\}\end{array}$ & - & $\begin{array}{l}\text { TS: }\left\{\begin{array}{cc}g & g+2 \\
1355 & 2\end{array}\right\} \\
\text { AS: }\left\{\begin{array}{cc}g & g+2 \\
1149 & 2\end{array}\right\}\end{array}$ \\
\hline$b=6$ & - & - & TS: $\left\{\begin{array}{l}g \\
5 \\
g \\
4\end{array}\right\}$ & - & $\begin{array}{c}\text { TS: }\left\{\begin{array}{cc}g & g+4 \\
222 & 1\end{array}\right\} \\
\text { AS: }\left\{\begin{array}{c}g \\
165\end{array}\right\}\end{array}$ & - \\
\hline$b=7$ & - & AS: $\left\{\begin{array}{l}g \\
1 \\
g \\
1\end{array}\right\}$ & - & $\begin{array}{l}\left.\text { TS: }: \begin{array}{c}g \\
37\end{array}\right\} \\
\text { AS: }\left\{\begin{array}{c}g \\
23\end{array}\right\}\end{array}$ & - & $\begin{array}{c}\text { TS: }\left\{\begin{array}{ccccc}g & g+2 & g+4 & g+6 & \text { NA } \\
3768 & 9 & 6 & 1 & 3\end{array}\right\} \\
\text { AS: }\left\{\begin{array}{ccc}g & g+2 & g+4 \\
2533 & 7 & 1\end{array}\right\}\end{array}$ \\
\hline$b=8$ & TS: $\left\{\begin{array}{l}g \\
1 \\
g \\
1\end{array}\right\}$ & - & $\begin{array}{l}\text { TS: }\left\{\begin{array}{l}g \\
8\end{array}\right\} \\
\text { AS: }\left\{\begin{array}{l}g \\
5\end{array}\right\}\end{array}$ & - & $\begin{array}{c}\left.\text { TS: } \begin{array}{cccc}g & g+2 & g+4 & \mathrm{NA} \\
453 & 5 & 2 & 1\end{array}\right\} \\
\text { AS: }\left\{\begin{array}{cc}g & g+2 \\
249 & 3\end{array}\right\}\end{array}$ & , \\
\hline$b=9$ & - & TS: $\left\{\begin{array}{l}g \\
2 \\
g \\
1\end{array}\right\}$ & - & $\begin{aligned} & \text { TS: }\left\{\begin{array}{cc}g & g+2 \\
61 & 1\end{array}\right\} \\
& \text { AS: }\left\{\begin{array}{c}g \\
25\end{array}\right\}\end{aligned}$ & 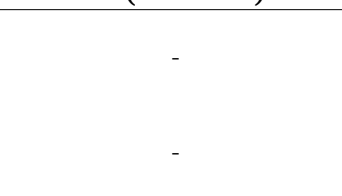 & $\begin{array}{c}\text { TS: }\left\{\begin{array}{ccccc}g & g+2 & g+4 & g+6 & \mathrm{NA} \\
6957 & 66 & 43 & 7 & 19\end{array}\right\} \\
\text { AS: }\left\{\begin{array}{ccccc}g & g+2 & g+4 & \mathrm{NA} \\
3243 & 33 & 7 & 1\end{array}\right\}\end{array}$ \\
\hline
\end{tabular}


different classes of ETSs in $\mathcal{T}$.

Table 8.6 shows that except for a small fraction of ETS structures, all the rest of the structures of ETS classes with size less than 10 and with less than 10 unsatisfied check nodes satisfy the LSS property. In particular, all the structures of size less than 10 with less than 5 unsatisfied check nodes are LSSs of 6-cycles.

The Case $d_{l}=5, g=8$

Theorem 12. For left-regular graphs with $d_{l}=5$ and $g=8$, the multiplicity of non-isomorphic LSS structures for different values of $x$ are listed in Table 8.7 for different classes of ETSs in $\mathcal{T}$.

Tables 8.5 and 8.7, in comparison with their counterparts for $g=6$, show that in graphs with larger girth, small trapping sets with small number of unsatisfied check nodes cannot exist. In particular, Table 8.7 indicates that in left-regular graphs with $d_{l}=5$ and $g=8$ no $(a, b)$ ETS with $a \leq 7$ and $b<10$ can exist. The inspection of Table 8.6 however, reveals that many of these ETS classes can exist in left-regular graphs with $d_{l}=5$ and $g=6$. The results of Table 8.7 also demonstrate that, for a left-regular graph with $d_{l}=5$ and $g=8$, all the $(a, b)$ ETSs with $a<10$ and $b<10$ are LSSs of 8-cycles.

Remark 15. It can be proved that it is not possible to have any $(a, b)$ ETS with $a<10$ and $b<10$ in a left-regular graph with $d_{l}=5$ and $g>8$.

The Case $d_{l}=6, g=6$

Theorem 13. For left-regular graphs with $d_{l}=6$ and $g=6$, the multiplicity of non-isomorphic LSS structures for different values of $x$ are listed in Table 8.8 for different classes of ETSs in $\mathcal{T}$.

The results of Table 8.8 indicate that for left-regular graphs with $d_{l}=6$ and $g=6$, all the possible $(a, b)$ absorbing sets with $a<10$ and $b \leq 10$ satisfy the LSS property with respect to 6-cycles. Moreover, except for one structure (out of 5411 structures) of the ETS class $(9,10)$, all the structures of $(a, b)$ ETS classes with $a<10$ and $b \leq 10$ are LSSs of short cycles. (The structure that does not satisfy the LSS property is shown in Figure 8.18.) Among these classes (excluding the $(9,10)$ class), 
Table 8.7: LSS Properties of Non-isomorphic Structures of $(a, b)$ ETS Classes for Leftregular Graphs with $d_{l}=5$ and $g=8$.

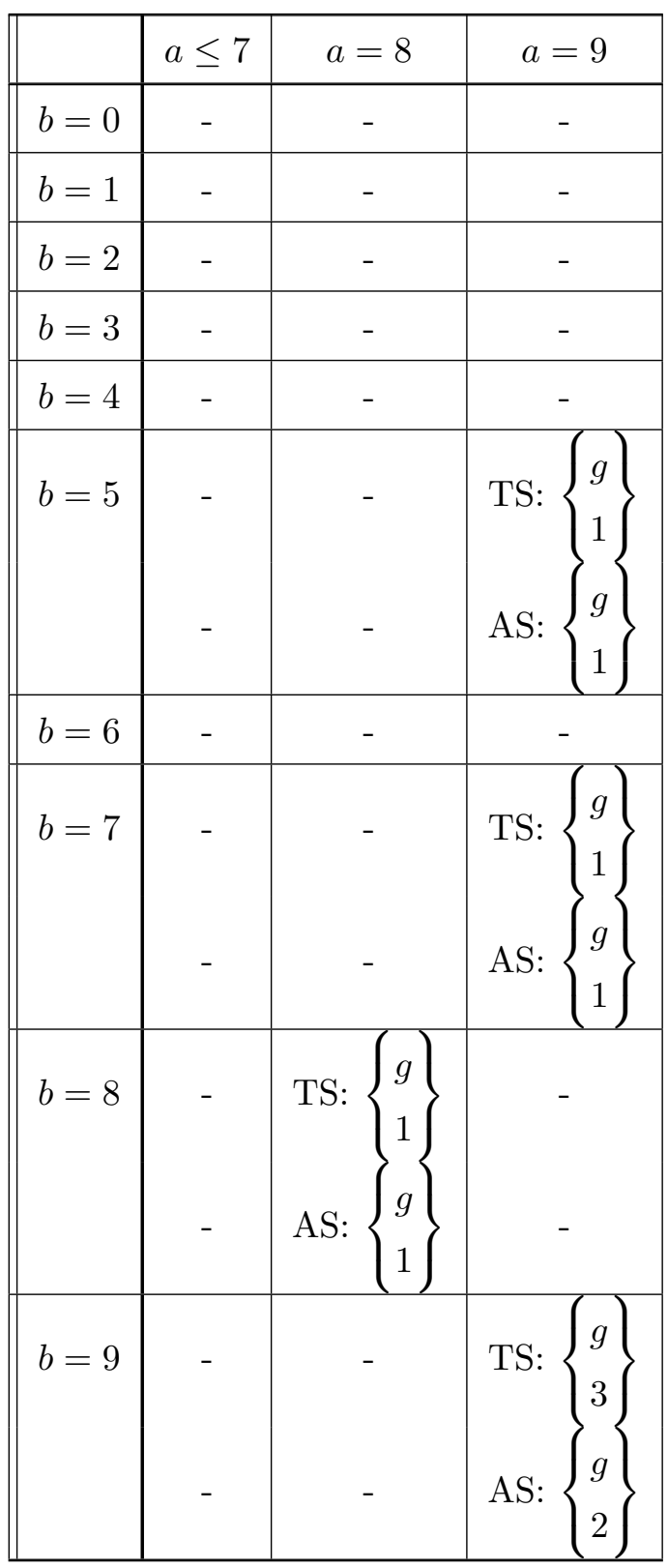

only one structure out of the 2274 possible structures for the $(9,8)$ ETS class is an LSS of 10-cycles. All the other possible structures for all the ETS classes are LSSs of 6-cycles. 
Table 8.8: LSS Properties of Non-isomorphic Structures of $(a, b)$ ETS Classes for Left-regular Graphs with $d_{l}=6$ and $g=6$.

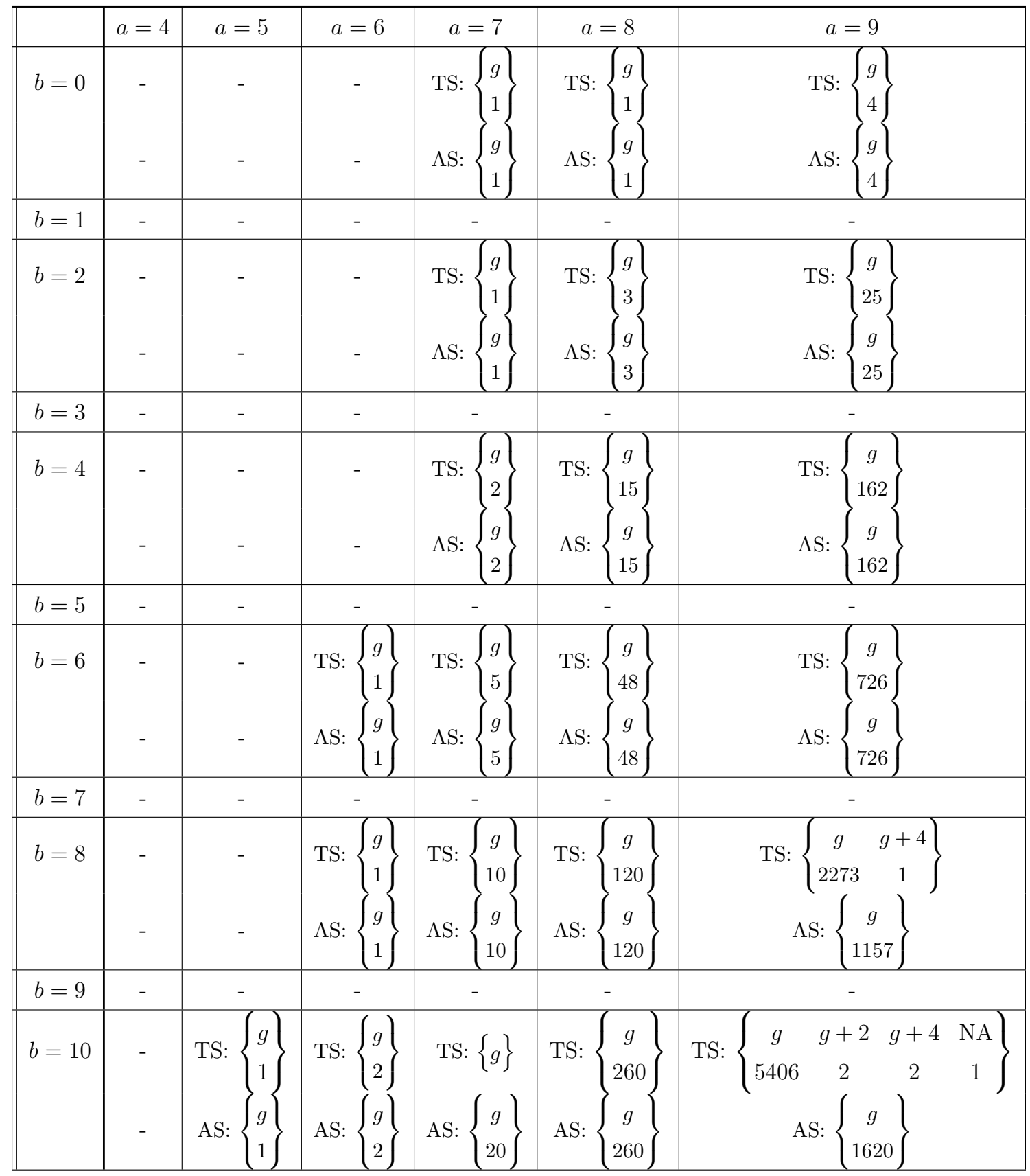

The Case $d_{l}=6, g>6$

Theorem 14. For left-regular graphs with $d_{l}=6$ and $g>6$, there does not exist any $(a, b)$ ETS in $\mathcal{T}$ with $a<10$ and $b \leq 10$. 


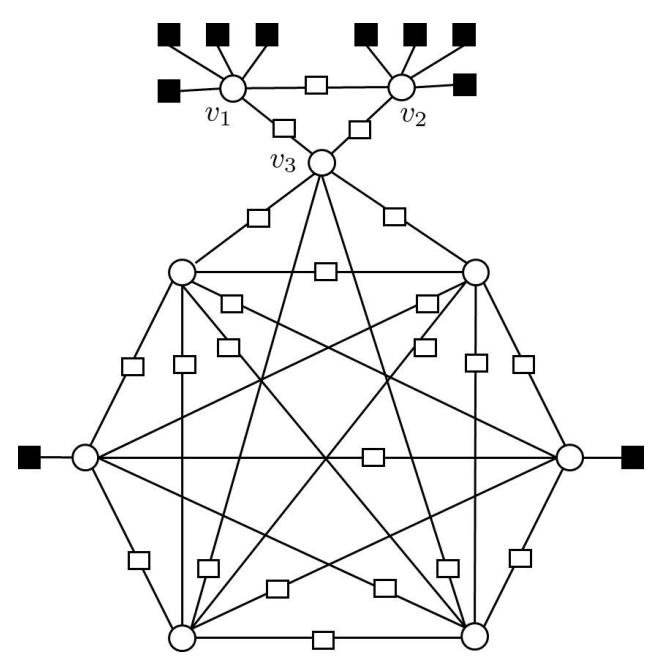

Figure 8.18: The only possible structure for a $(9,10)$ ETS in a left-regular graph with $d_{l}=6$ and $g=6$ that does not satisfy the LSS property. 


\section{Chapter 9}

\section{Conclusions and Future Works}

\subsection{Conclusion}

We proposed a distributed message-passing algorithm to count short cycles in a graph. For bipartite graphs, the proposed algorithm counts short cycles of length $g, g+2, \ldots, 2 g-2$, where $g$ is the girth of the graph. For non-bipartite graphs, the algorithm counts cycles of length $g, g+1, \ldots, 2 g-1$. The operations performed by the algorithm are integer additions and subtractions, and the computational and storage complexities of the algorithm are $O\left(g|E|^{2}\right)$ and $O\left(d_{\text {max }}|E|\right)$, respectively, where $|E|$ and $d_{\text {max }}$ are the number of edges and the maximum node degree in the graph, respectively. For sparse graphs, the proposed algorithm is significantly faster and requires substantially less memory compared to the existing algorithms, such as that of [36], which are tailored for counting short cycles. This is particularly the case for larger graphs.

Interestingly, the more generic and basic approach of matrix multiplication, when properly implemented, has a complexity similar to the proposed message-passing algorithm, and is thus less complex than the approaches specifically tailored for counting short cycles, in particular for large sparse graphs. We demonstrated that the efficient implementation of matrix multiplication approach can also be described as a forward message-passing algorithm over a trellis diagram. Both the forward message-passing algorithm and the one proposed in this work in fact count the tailless backtrackless closed (tbc) walks in the graph, which happen to coincide with the simple cycles as long as their length is less than twice the girth of the graph. The difference however is that to limit the closed walks to tbc walks, the former operates on a properly defined graph (matrix), i.e., the trellis associated with the directed edge matrix, while the 
latter employs the extrinsic property of the message-passing over the original graph.

While in this thesis, our main focus was on counting short simple cycles, there may be applications where one is interested in counting the tbc walks in a graph. One example is the characterization of pseudo-codewords of iterative coding schemes [62]. In such applications, the proposed message-passing algorithm can be applied with no limitation on the length of the closed walks.

We also presented a method for counting short cycles in the Tanner graphs of QC protograph LDPC codes. The method uses the relationships between the number of short cycles in a graph with the eigenvalues of the directed edge matrix of the graph. We showed that the complexity of computing such eigenvalues can be reduced significantly by representing the directed edge matrix as a block circulant matrix. For the Tanner graphs of QC protograph LDPC codes, the proposed algorithm is faster compared to the existing algorithms.

For a given base graph, we derived lower bounds on the lifting degree of quasi-cyclic protograph LDPC codes required to achieve a certain girth. The bounds, which can also be interpreted as upper bounds on the girth of the lifted graphs for a given base graph and a given lifting degree, were obtained by examining the dependencies among the permutation shifts of certain tailless backtrackless closed walks of the base graph. The bounds derived in this work, which are applicable to both regular and irregular base graphs with no parallel edges, are in some cases more general and in some other cases tighter than the existing bounds. The results presented in this work can be used to design cyclic liftings with relatively small degree and relatively large girth. As an example, we presented new quasi-cyclic protograph LDPC code constructions with girth 8 based on fully-connected base graphs. These constructions provide upper bounds on the required lifting degree to achieve girth 8 using fully-connected base graphs.

We also proposed an efficient algorithm for finding the dominant trapping sets of an LDPC code. The algorithm starts from an initial set of trapping sets and recursively and greedily expands them to trapping sets of larger size. The initial set for regular codes is a set of short cycles, and for irregular codes, it also includes variable nodes of small degree and cycles with low ACE values. To devise the expansions, the structure of dominant trapping sets is carefully studied for both regular and irregular codes. The efficiency and accuracy of the proposed algorithm was demonstrated through a number of examples. It was observed that the proposed algorithm is faster 
by up to about two orders of magnitude compared to similar search algorithms.

We also investigated the structure of elementary trapping sets (ETS) of left-regular LDPC codes. We developed an approach to find all the non-isomorphic structures of a given $(a, b)$ class of ETSs, where $a$ is the size and $b$ is the number of unsatisfied check nodes of the ETS. For left-regular LDPC codes with left degrees $d_{l}=3,4,5,6$, and girths $g=6,8$, we studied such structures and demonstrated that an overwhelming majority of them are layered supersets (LSS) of short cycles in the Tanner graph of the code. In particular, we proved that for any category of left-regular LDPC codes with given $d_{l}$ and $g$, there exist integers $\alpha$ and $\beta$ such that all the classes of $(a, b)$ ETSs with $a<\alpha$ and $b<\beta$, are LSSs of short cycles. This implies that for any category of left-regular LDPC codes, the dominant ETSs are all LSSs of short cycles. The LSS characterization of dominant ETSs is particularly important as it corresponds to a simple algorithm that can find all such ETSs in a guaranteed fashion starting from the short cycles of the graph. For any class of $(a, b)$ ETSs, the lengths of the required short cycles were provided in this thesis.

One important contribution of this part of the thesis is the approach developed to exhaustively find all the non-isomorphic structures of a given class of $(a, b)$ ETSs for arbitrary values of $a$ and $b$ and for left-regular LDPC codes of arbitrary left degree and girth. In a more general context, the database of such structures can be very helpful in the analysis and the design of LDPC codes with low error floor. In particular, one can use this information to find all the ETSs of a certain class in a guaranteed fashion regardless of whether those ETSs satisfy the LSS property or not. To the best of our knowledge, the results presented in Tables 8.1 - 8.8 are the most comprehensive results available so far on the structure of ETSs of regular LDPC codes.

The results of this thesis have been published or submitted for publication in [47], [48], [49], [50], [51], [52], [53], [54] and [55].

\subsection{Future Works}

Insights gained from this work can enable further advancement of analysis and design of LDPC codes. As an example, a database of non-isomorphic structures of elementary trapping sets can be formed using the approach developed in this work. Such data base can be used in the analysis and the design of regular LDPC codes with low error floors. 
The characterization of dominant trapping sets can be extended to irregular LDPC codes, or be customized for the structured LDPC codes such as protograph LDPC codes.

The links established in this work between a dominant trapping set and its subsets and short cycles can be used to design LDPC codes with low error floors.

The bounds derived in this work on the girth of quasi-cyclic protograph LDPC codes can be extended to a wider range of LDPC codes (e.g., protograph LDPC codes whose base graphs have multiple edges) and a wider range of degree distributions. Our results revealed that for larger left degrees, the current bounds are not necessarily very tight, and there is still room for improvement. 


\section{List of References}

[1] N. Alon, R. Yuster and U. Zwick, "Finding and counting given length cycles," Algorithmica, vol. 17 no. 3, pp. 209-223, 1997.

[2] R. Asvadi, A. H. Banihashemi and M. Ahmadian-Attari, "Lowering the error floor of LDPC codes using cyclic liftings," IEEE Trans. Inform. Theory, vol. 57, no. 4, pp. 2213-2224, Apr. 2011.

[3] R. Asvadi, A. H. Banihashemi and M. Ahmadian-Attari, "Design of irregular quasi-cyclic protograph codes with low error floors," IEEE Trans. Communications, vol. 60, no. 4, pp. 902-907. Apr. 2012.

[4] S. Abu-Surra, D. DeClercq, D. Divsalar and W. Ryan, "Trapping set enumerators for specific LDPC codes," Proc. Inform. Theory and Applications Workshop, San Diego, CA, Jan. 31- Feb. 5, 2010, pp. 1-5.

[5] S. Abu-Surra, W. E. Ryan, D. Divsalar and W. Ryan, "Enumerators for protograph-based ensembles of LDPC and generalized LDPC codes," IEEE Trans. Inform. Theory, vol. 57, no. 2, pp. 858-886, Feb. 2011.

[6] E. T. Bax, "Algorithms to count paths and cycles," Information Processing Letters, vol. 52, no. 5, pp. 249-252, Dec. 1994.

[7] C. Berrou, A. Glavieux and P. Thitimajshima, "Near Shannon limit errorcorrecting coding and decoding: Turbo-codes," in Proc. IEEE Int. Conf. Communications, Geneva, Switzerland, pp. 1064-1070, May 1993.

[8] I. E. Bocharova, F. Hug, R. Johannesson, B. D. Kudryashov and R. V. Satyukov, "Some voltage graph-based LDPC tailbiting codes with large girth," Proc. IEEE International Symposium on Inform. Theory (ISIT'11), St. Petersburg, Russia, Jul. 31 - Aug. 5, 2011, pp. 732-736.

[9] I. E. Bocharova, F. Hug, R. Johannesson, B. D. Kudryashov and R. V. Satyukov, "Searching for voltage graph-based LDPC tailbiting codes with large girth," CoRR, arxiv.org/abs/1108.0840.

[10] S. ten Brink, G. Kramer and A. Ashikhmin, "Design of low-density parity-check codes for modulation and detection", IEEE Trans. on Communications, vol 52, pp. 670-678, Apr. 2004. 
[11] E. Cavus and B. Daneshrad, "A performance improvement and error floor avoidance technique for belief propagation decoding of LDPC codes," Proc. 16th IEEE International Symposium Pers. Indoor Mobile Radio Communications, Los Angeles, CA, Sept. 2005, vol. 4, pp. 2386-2390.

[12] G. G. Cash, "The number of $n$-cycles in a graph," Applied Mathematics and Computation, vol. 184, no. 2, pp. 1080-1083, Jan. 2007.

[13] Y. C. Chang and H.L. Fu, "The number of 6-cycles in a graph," Bull. Inst. Combin. Appl. vol. 39, pp. 27-30, Sept. 2003.

[14] R. Chen, H. Huang and G. Xiao, "Relation between parity-check matrices and cycles of associated Tanner graphs," IEEE Comm. Lett., vol. 11, no. 8, pp. 674 - 676, Aug. 2007.

[15] S. Y. Chung, "On the construction of some capacity-approaching coding schemes," Ph.D. thesis, Dept. of Electrical Engineering and Computer Science, Massachusetts Institute of Technology, Sept. 2000.

[16] C. Cole, S. Wilson, E. Hall and T. Giallorenzi, "A general method for finding low error rates of LDPC codes," CoRR, arxiv.org/abs/cs/0605051.

[17] D. Coppersmith and S. Winograd, "Matrix multiplication via arithmetic progressions," Journal of Symbolic Computation, vol. 9, pp. 251-280, 1990.

[18] C. Di, D. Proietti, I. E. Telatar, T. J. Richardson and R. L. Urbanke, "Finitelength analysis of low-density parity-check codes on the binary erasure channel," IEEE Trans. Inform. Theory, vol. 48, no. 6, pp. 1570-1579, June 2002.

[19] Qiuju Diao, Ying Yu Tai, Shu Lin and Khaled A. S. Abdel-Ghaffar, "Trapping set structure of finite geometry LDPC codes," Proc. IEEE International Symposium on Inform. Theory (ISIT'12), MIT, Cambridge, MA, USA, July 1-6, 2012, pp. 3088-3092.

[20] Q. Diao, Q. Huang, S. Lin and K. Abdel-Ghaffar, "A matrix theoretic approach for analyzing quasi-cyclic low-density parity-check codes," IEEE Trans. Inform. Theory, vol. 58, no. 6, pp. 4030-4048, June 2012.

[21] L. Dolecek, Z. Zhang, M. Wainwright, V. Anantharam and B. Nikolic, "Evaluation of the low frame error rate performance of LDPC codes using importance sampling," Proc. IEEE Inform. Theory Workshop, Lake Tahoe, CA, Sept. 2-6, 2007, pp. 202-207.

[22] L. Dolecek, P. Lee, Z. Zhang, V. Anantharam, B. Nikolic and M. Wainwright, "Predicting error floors of LDPC codes: deterministic bounds and estimates," IEEE Journal on Selected Areas in Communications, vol. 27, no. 6, pp. 908-917, Aug. 2009.

[23] L. Dolecek, Z. Zhang, V. Anantharam, M. J. Wainwright and B. Nikolic, "Analysis of absorbing sets and fully absorbing sets of array-based LDPC codes," IEEE Trans. Inform. Theory, vol. 56, no. 1, pp. 181-201, Jan. 2010. 
[24] ETSI Standard TS 102 005: Digital Video Broadcasting (DVB): frame structure channel coding and modulation for a second generation digital terrestrial television broadcasting system (DVB-T2), ETSI Std. TS 102 005, March 2010.

[25] ETSI Standard TR 102376 V1.1.1: Digital Video Broadcasting (DVB) User guidelines for the second generation system for Broadcasting, Interactive Services, News Gathering and other broadband satellite applications (DVBS2), ETSI Std. TR 102 376, Feb. 2005.

[26] E. Eleftheriou and S. Olcer, "Low density parity check codes for digital subscriber lines," Proc. IEEE International Conference on Communications, New York, Apr. May 2002, pp. 1752-1757.

[27] M. Esmaeili and M. Gholami, "Structured quasi-cyclic LDPC codes with girth 18 and column-weight $\mathrm{J}=3$," International Journal of Electronics and Communications (AEU), vol. 64, no. 3, pp. 202-217, Mar. 2010.

[28] T. Etzion, A. Trachtenberg and A. Vardy, "Which codes have cycle-free Tanner graphs?," IEEE Trans. Inform. Theory, vol. 45, no. 6, pp. 2173-2181, Sept. 1999.

[29] J. Fan and Y. Xiao, "A method of counting the number of cycles in LDPC codes," 8th Int. Conf. Signal Proc. (ICSP), vol. 3, pp. 2183-2186, 2006.

[30] J. L. Fan, "Array codes as low-density parity-check codes", Proc. 2nd International Symposium on Turbo Codes and their applications, Brest, France, Sept. 2000, pp. 543-546.

[31] J. Flum and M. Grohe, "The parameterized complexity of counting problems," in Proc. IEEE Symp. Foundations of Computer Science, Vancouver, BC, Canada, pp. 538-547, Nov. 2002.

[32] M. P. C. Fossorier, "Quasi-cyclic low-density parity-check codes from circulant permutation matrices," IEEE Trans. Inform. Theory, vol. 50, no. 8, pp. 17881793, Aug. 2004.

[33] R. G. Gallager, "Low-density parity-check codes," IEEE Trans. Inform. Theory, vol. 8, no. 1, pp. 21-28, Jan. 1962

[34] J.L. Gross and T.W. Tucker, Topological Graph Theory, Wiley, NY, 1987.

[35] Y. Han and W. E. Ryan, "Low-floor decoders for LDPC codes," IEEE Trans. Communications, vol. 57, pp. 1663-1673, June. 2009.

[36] T. R. Halford and K. M. Chugg, "An algorithm for counting short cycles in bipartite graphs," IEEE Trans. Inform. Theory, vol. 52, no. 1, pp. 287-292, Jan. 2006.

[37] T. Halford, K. Chugg and A. Grant, "Which codes have 4-cycle-free Tanner graphs," Proc. IEEE International Symposium on Inform. Theory (ISIT'06), Seattle, WA, Jul. 2006, pp. 871-875.

[38] M. Horton, "Ihara Zeta Functions of Irregular Graphs," Ph.D. Thesis, U.C.S.D., 2006. 
[39] X.-Y. Hu, E. Eleftheriou and D. M. Arnold, "Regular and irregular progressive edge-growth Tanner graphs," IEEE Trans. Inform. Theory, vol. 51, no. 1, pp. 386-398, Jan. 2005.

[40] Qin Huang, Qiuju Diao, Shu Lin and Khaled A. S. Abdel-Ghaffar, "Cyclic and quasi-cyclic LDPC codes on constrained parity-check matrices and their trapping sets," IEEE Trans. Inform. Theory, vol. 58, no. 5, pp. 2648-2671, May 2012.

[41] IEEE-802.11n. Wireless LAN Medium Access Control and Physical Layer Specifications: Enhancements for Higher Throughput. P802.11n/D3.07, March 2008.

[42] IEEE Standard for Local and Metropolitan Area Networks Part 16: Air Interface for Fixed and Mobile Broadband Wireless Access Systems Amendment 2: Physical and Medium Access Control Layers for Combined Fixed and Mobile Operation in Licensed Bands and Corrigendum 1, IEEE Std. 802.16e, Feb. 2006.

[43] IEEE P802.22 Draft Standard for Wireless Regional Area Networks Part 22: Cognitive Wireless RAN Medium Access Control (MAC) and Physical Layer (PHY) Specifications: Policies and Procedures for Operation in the TV Bands, IEEE P802.22 Draft Standard, D0.3, May 2007.

[44] IEEE Standard for Information Technology-Telecommunications and Information Exchange between Systems-Local and Metropolitan Area Networks-Specific Requirements Part 3: Carrier Sense Multiple Access with Collision Detection (CSMA/CD) Access Method and Physical Layer Specifications, IEEE Std. 802.3an, Sept. 2006.

[45] M. Ivkovic, S. K. Chilappagari and B. Vasic, "Eliminating trapping sets in lowdensity parity-check codes by using Tanner graph covers," IEEE Trans. Inform. Theory, vol. 54, no. 8, pp. 3763-3768, Aug. 2008.

[46] D. B. Johnson, "Find all the elementary circuits of a directed graph," Journal SIAM, vol. 4, pp. 77-84, 1975.

[47] M. Karimi Dehkordi and A. H. Banihashemi, "A message-passing algorithm for counting short cycles in a graph," Proc of 2010 IEEE Inform. Theory Workshop, Cairo, Egypt, Jan. 6 - 8, 2010, pp. 1-5.

[48] M. Karimi and A. H. Banihashemi, "An efficient algorithm for finding dominant trapping sets of LDPC codes," Proc. 6th International Symposium on Turbo Codes $\mathscr{E}$ Iterative Information Processing, Brest, France, Sept. 6-10, 2010, pp. 444-448.

[49] M. Karimi and A. H. Banihashemi, "An efficient algorithm for finding dominant trapping sets of irregular LDPC codes," Proc. IEEE International Symposium on Inform. Theory (ISIT'11), Saint-Petersburg, Russia, July 31 - August, 5, 2011, pp. 1091-195.

[50] M. Karimi and A. H. Banihashemi, "On the girth of quasi cyclic protograph LDPC codes," Proc. IEEE International Symposium on Inform. Theory (ISIT'12), MIT, Cambridge, MA, USA, July 1-6, 2012, pp. 3078-3082. 
[51] M. Karimi and A. H. Banihashemi, "A message-passing algorithm for counting short cycles in a graph," IEEE Trans. Communications, vol. 61, no. 2, pp. 485495, Feb. 2013.

[52] M. Karimi and A. H. Banihashemi, "Efficient algorithm for finding dominant trapping sets of LDPC codes," IEEE Trans. Inform. Theory, vol. 58, pp. 69426958, Nov. 2012.

[53] M. Karimi and A. H. Banihashemi, "Counting short cycles of quasi cyclic protograph LDPC codes," IEEE Comm. Lett., vol. 16, no. 3, pp. 400- 403, March 2012.

[54] M. Karimi and A. H. Banihashemi, "On the girth of quasi cyclic protograph LDPC codes," IEEE Trans. Inform. Theory, vol. 59, no. 7, pp. 4542-4552, Jul. 2013.

[55] M. Karimi and A. H. Banihashemi, "On characterization of elementary trapping sets of variable-regular LDPC codes," submitted to IEEE Trans. Inform. Theory.

[56] C. A. Kelley and J. L. Walker, "LDPC codes from voltage graphs," Proc. IEEE International Symposium on Inform. Theory (ISIT08), Toronto, Canada, 6-11 Jul. 2008, pp.792-796.

[57] C. A. Kelley, "On codes designed via algebraic lifts of graphs," Proc. 46th Annual Allerton Conference on Communication, Control and Computing,, UrbanaChampaign, IL, Sept. 2008, pp. 583-590.

[58] S.Kim, J.-S.No, H.Chung and D.-J.Shin, "On the girth of Tanner $(3,5)$ quasicyclic LDPC codes," IEEE Trans. Inform. Theory, vol. 52, no. 4, pp. 1729-1744, Apr. 2006.

[59] S. Kim, J.-S. No, H. Chung and D.-J. Shin, "Quasi-cyclic low density paritycheck codes with girth larger than 12," IEEE Trans. Inform. Theory, vol. 53, no. 8, pp. 2885-2891, Aug. 2007.

[60] R. Koetter and P. O. Vontobel, "Graph-covers and iterative decoding of finite length codes," Proc. IEEE International Symposium on Turbo Codes and Applications, Brest, France, Sept. 2003, pp. 75-82.

[61] R. Koetter, W.C.W. Li, P.O. Vontobel and J.L. Walker, "Pseudo-codewords of cycle codes via zeta functions," Proc. IEEE Inform. Theory Workshop, San Antonio, TX, USA, 2004, pp. 7-12.

[62] R. Koetter, W.C.W. Li, P. O. Vontobel and J. L. Walker, "Characterizations of pseudo-codewords of (low-density) parity-check codes," Advances in Mathematics, vol. 213, no. 1, pp. 205-229, 2007.

[63] C. Koller, A. Graell i Amat, J. Kliewer and D. J. Costello, "On trapping sets for repeat accumulate accumulate codes," Proc. Inform. Theory and Applications Workshop, San Diego, La Jolla, CA, Feb. 8-13, 2009, pp. 164-168. 
[64] C. Koller, A. Graell i Amat, J. Kliewer and D. J. Costello, "Trapping set enumerators for repeat multiple accumulate code ensembles," Proc. IEEE International Symposium on Inform. Theory (ISIT'09), Seoul, Korea, Jun. - Jul. 2009, pp. 1819-1823.

[65] K. M. Krishnan and P. Shankar, "Computing the stopping distance of a Tanner graph is NP-hard," IEEE Trans. Inform. Theory, vol. 53, no. 6, pp. 2278-2280, June 2007.

[66] F. R. Kschischang, B. J. Frey and H.-A. Loeliger "Factor graphs and the sumproduct algorithm," IEEE Trans. Inform. Theory, vol. 47, no. 2, pp. 498-519, Feb. 2001.

[67] G. B. Kyung and C.-C. Wang, "Finding the exhaustive list of small fully absorbing sets and designing the corresponding low error-floor decoder," IEEE Trans. Communications, vol. 60, pp. 1487-1498, June 2012.

[68] G. B. Kyung, "private communication."

[69] S. Laendner and O. Milenkovic, "Algorithmic and combinatorial analysis of trapping sets in structured LDPC codes," Proc. IEEE International Conference on Wireless Networks, Communications and Mobile Computing, Hawaii, USA, June 13-16, 2005, pp. 630-635.

[70] S. Laendner, T. Hehn, O. Milenkovic and J.B. Huber, "The trapping redundancy of linear block codes," IEEE Trans. Inform. Theory, vol. 55, no. 1, pp. 53-63, Jan. 2009.

[71] S. Laendner, T. Hehn, O. Milenkovic and J.B. Huber, "Characterization of small trapping sets in LDPC codes from Steiner triple systems," Proc. 6th International Symposium on Turbo Codes $\&$ Iterative Information Processing, Brest, France, Sept. 6 - 10, 2010, pp. 93-97.

[72] Y. Mao and A.H. Banihashemi, "A heuristic search for good low-density paritycheck codes at short block lengths," Proc. IEEE International Conference on Communications, Helsinki, Finland, June 2001, pp. 41-44.

[73] B. D. McKay, N. C. Wormald and B. Wysocka, "Short cycles in random regular graphs," Electronic Journal of Combinatorics, vol. 11, no. R66, 2004.

[74] D. J. C. MacKay and M. S. Postol, "Weaknesses of Margulis and RamanujanMargulis low-density parity-check codes," Electronic Notes in Theoretical Computer Science, vol. 74, pp. 97-104, Oct. 2003.

[75] G. A. Margulis, "Explicit constructions of graphs without short cycles and low density codes," Combinatorica, vol. 2, no. 1, pp. 71-78, 1982.

[76] A. McGregor and O. Milenkovic, "On the hardness of approximating stopping and trapping sets," IEEE Trans. Inform. Theory, vol. 56, no. 4, pp. 1640-1650, Apr. 2010. 
[77] O. Milenkovic, N. Kashyap and D. Leyba, "Shortened array codes of large girth," IEEE Trans. Inform. Theory, vol. 52, no. 8, pp. 3707-3722, Aug. 2006.

[78] O. Milenkovic, E. Soljanin and P. Whiting, "Asymptotic spectra of trapping sets in regular and irregular LDPC code ensembles," IEEE Trans. Inform. Theory, vol. 53, no. 1, pp. 39-55, Jan. 2007.

[79] K. Murali Krishnan and L. Sunil Chandran, "Hardness of approximation results for the problem of finding the stopping distance in Tanner graphs," Proc. 26th International Conference on Foundations of Software Technology and Theoretical Computer Science, Kolkata, India, Dec. 13-15, 2006, pp. 69-80.

[80] S. Myung, K. Yang and J. Kim, "Quasi-cyclic LDPC codes for fast encoding," IEEE Trans. Inform. Theory, vol. 51, no. 8, pp. 2894-2901, Aug. 2005.

[81] D. V. Nguyen, S. K. Chilappagari, M. W. Marcellin and B. Vasic, "On the construction of structured LDPC codes free of small trapping sets," IEEE Trans. Inform. Theory, vol. 58, no. 4, pp. 2280-2302, Apr. 2012.

[82] A. Otmani, J.-P. Tillich and I. Andriyanova, "On the minimum distance of generalized LDPC codes," Proc. IEEE International Symposium on Inform. Theory, Nice, France, June 24-29, 2007, pp. 751-755.

[83] E. Pusane, D. J. Costello and D. G.M. Mitchell, "Trapping set analysis of protograph-based LDPC convolutional codes," Proc. IEEE International Symposium on Inform. Theory (ISIT'09), Seoul, Korea, Jun. - Jul. 2009, pp. 561-565.

[84] T. J. Richardson and R. Urbanke, "The capacity of low-density paritycheck codes under message-passing decoding," IEEE Trans. Inform. Theory, vol. 47, no. 2, pp. 599-618, Feb. 2001.

[85] T. J. Richardson, M. A. Shokrollahi and R. L. Urbanke, "Design of capacityapproaching irregular low density parity-check codes," IEEE Trans. Inform. Theory, vol. 47, no. 2, pp. 619-637, Feb. 2001.

[86] T. Richardson, "Error floors of LDPC codes," Proc. 41th Annual Allerton Conference on Communication, Control and Computing, Monticello, IL, Oct. 2003, pp. 1426-1435.

[87] T. Richardson and R. Urbanke, Modern Coding Theory. Cambridge University Press, 2008.

[88] E. Rosnes and O. Ytrehus, "An efficient algorithm to find all small-size stopping sets of low-density parity-check matrices," IEEE Trans. Inform. Theory, vol. 55, no. 9, pp. 4167-4178, Sept. 2009.

[89] A. Sakzad, M.-R. Sadeghi and D. Panario, "Codes with Girth 8 Tanner Graph Representation," Design Codes and Cryptography, vol. 57, pp. 71-81, 2010.

[90] C. Schlegel and S. Zhang, "On the dynamics of the error floor behavior in (regular) LDPC codes," IEEE Trans. Inform. Theory, vol. 56, no. 7, pp. 3248-3264, June 2010. 
[91] R. Smarandache and P. O. Vontobel, "Quasi-Cyclic LDPC Codes: Influence of Proto- and Tanner-Graph Structure on Minimum Hamming Distance Upper Bounds," Jan. 26, 2009 [Online]. Available: http://arxiv.org/pdf/0901.4129v1

[92] G. S. Staples, "A new adjacency matrix for finite graphs," Proc. 7th International Conference on Cliford Algebras and Their Applications, Toulouose, France, May 2005.

[93] R. Schott and G. S. Staples, "Nilpotent adjacency matrices, random graphs and quantum random variables," J. Phys. A: Math. Theor., vol. 41 (2008), 155-205.

[94] R. Schott and G. S. Staples, "On the complexity of cycle enumeration using zeons," Proc. Applied Geometric Algebras in Computer Science and Engineering 2010, Amsterdam, June 14 - 16, 2010, pp. 1-22.

[95] R. Schott and G. S. Staples, "Cycle enumeration using nilpotent adjacency matrices with algorithm runtime comparisons," available at www.loria.fr/ schott/CVRENE3html.html.

[96] H. M. Stark and A. A. Terras, "Zeta Functions of finite graphs and coverings," Advances in Math., vol. 121 (1996), pp. 124-165.

[97] C. Storm, "Some properties of graphs determined by edge zeta functions," Linear Algebra and its Applications, vol. 434, no. 5, pp. 1285-1294, March 2011.

[98] S. Huss-Lederman, E. M. Jacobson, A. Tsao, T. Turnbull and J. R. Johnson, "Implementation of Strassen's algorithm for matrix multiplication," Proc. of ACM/IEEE conference on Supercomputing, pp. 32, 1996.

[99] M. E. O'Sullivan, "Algebraic construction of sparse matrices with large girth," IEEE Trans. Inform. Theory, vol. 52, no. 2, pp. 718-727, Feb. 2006.

[100] R. M. Tanner, D. Sridhara and T. Fuja, "A class of group-structured LDPC codes," Proc. International Symposium on Communication Theory and Applications, Ambleside, U.K., July 2001, pp. 365-370.

[101] R. M. Tanner, D. Sridhara, A. Sridharan, T. E. Fuja and J. D. J. Costello, "LDPC block and convolutional codes based on circulant matrices," IEEE Trans. Inform. Theory, vol. 50, no. 12, pp. 2966-2984, Dec. 2004.

[102] R. M. Tanner, "A recursive approach to low-complexity codes," IEEE Trans. Inform. Theory, vol. 27, no. 5, pp. 533-547, Sept. 1981.

[103] R. Tarjan, "Enumeration of the elementary circuits of a directed graph," J. SIAM, 2:211-216, 1973.

[104] G. J. Tee, "Eigenvectors of block circulant and alternating circulant matrices," Res. Lett. Inform. Math. Sci., vol. 8, pp. 123-142, 2005.

[105] T. Tian, C. Jones, J. D. Villasenor and R. D. Wesel, "Selective avoidance of cycles in irregular LDPC code construction," IEEE Trans. Communications, vol. 52, no. 8, pp. 1242-1248, Aug. 2004. 
[106] J.-P. Tillich and G. Zemor, "On the minimum distance of structured LDPC codes with two variable nodes of degree 2 per parity-check equation," Proc. IEEE International Symposium on Inform. Theory, Seattle, WA, July 9-14, 2006, pp. 1549-1553.

[107] J. Thorpe, "Low-density parity-check (LDPC) codes constructed from protograph," IPN Progress Report 42-154, JPL, Aug. 2003.

[108] R. Vandebril, M. Barel and N. Mastronardi, Matrix Computations and Semiseparable Matrices: Eigenvalue and singular value methods, JHU Press, 2009.

[109] B. Vasic and E. Kurtas, Coding and Signal Processing for Magnetic Recording Systems. Boca Raton, FL: CRC, 2005.

[110] B. Vasic, S. Chilappagari, D. Nguyen and S. Planjery, "Trapping set ontology," Proc. 47th Annual Allerton Conference on Communication, Control and Computing, Monticello, IL, Sept. 2009, pp. 1-7.

[111] P. O. Vontobel and R. Koetter, "Graph-cover decoding and finite-length analysis of message-passing iterative decoding of LDPC codes," CoRR, arxiv.org/abs/cs/0512078.

[112] D. Vukobratovic and V. Senk, "Generalized ACE constrained progressive edge growth LDPC code design," IEEE Communications Letters, vol. 12, no. 1, pp. 32-34, Jan. 2008.

[113] C. C. Wang, "On the exhaustion and elimination of trapping sets: Algorithms \& the suppressing effect," Proc. IEEE International Symposium on Inform. Theory, Nice, France, June 24-29, 2007, pp. 2271-2275.

[114] C. C. Wang, S.R. Kulkarni and H.V. Poor, "Finding all small error-prone substructures in LDPC codes," IEEE Trans. Inform. Theory, vol. 55, no. 5, pp. 1976-1998, May 2009.

[115] Y. Wang, J. S. Yedidia and S. C. Draper, "Construction of high-girth QC-LDPC codes," Proc. 5th International Symposium on Turbo Codes and Related Topics, Lausanne, Switzerland, 15 Sept. 2008, pp.180-185.

[116] D.B. West, Introduction to Graph Theory, Prentice-Hall, 1996.

[117] N. Wiberg, "Codes and Decoding on General Graphs", Ph.D. thesis, Linkoping University, Linkoping, Sweden, 1996.

[118] H. Xiao and A. H. Banihashemi, "Improved progressive-edge-growth (PEG) construction of irregular LDPC codes," IEEE Communications Letters, vol. 8, no. 12 , pp. 715-718, Dec. 2004.

[119] H. Xiao and A. H. Banihashemi, "Graph-based message-passing schedules for decoding LDPC codes," IEEE Trans. Communications, vol. 52, no. 12, pp. 2098 - 2105, Dec. 2004. 
[120] H. Xiao and A. H. Banihashemi, "Estimation of bit and frame error rates of lowdensity parity-check codes on binary symmetric channels," IEEE Trans. Communications, vol. 55, pp. 2234-2239, Dec. 2007.

[121] H. Xiao and A. H. Banihashemi, "Error rate estimation of low-density paritycheck codes on binary symmetric channels using cycle enumeration," IEEE Trans. Communications, vol. 57, pp. 1550-1555, June 2009.

[122] H. Xiao, A. H. Banihashemi and M. Karimi, "Error rate estimation of lowdensity parity-check codes decoded by quantized soft-decision iterative algorithms," IEEE Trans. Communications, vol. 61, pp. 474-483, Feb. 2013.

[123] J. Xu and S. Lin, "A combinatoric superposition method for constructing lowdensity parity-check codes," Proc. IEEE International Symposium on Inform. Theory (ISIT'03), Yokohama, Japan, June-Jul. 2003, p. 30.

[124] J. Xu, L. Chen, L.-Q. Zeng, L. Lan and S. Lin, "Construction of low density parity-check codes by superposition," IEEE Trans. Communications, vol. 53, no. 2, pp. 243-251, Feb. 2005.

[125] K. Yang and T. Helleseth, "On the minimum distance of array codes as LDPC codes," IEEE Trans. Inform. Theory, vol. 49, no. 12, pp. 3268-3271, Dec. 2003.

[126] M.Yang, W. E. Ryan and Y. Li, "Design of efficiently-encodable moderatelength high-rate irregular LDPC codes," IEEE Trans. Communications, vol. 52, no. 4, pp. 564-571, Apr. 2004.

[127] R. Yuster and U. Zwick, "Fast sparse matrix multiplication," ACM Transactions on Algorithms, vol. 1, pp. 2 - 13, 2004.

[128] Y. Zhang and W. E. Ryan, "Toward low LDPC-code floors: a case study," IEEE Trans. Communications, vol. 57, no. 6, pp. 1566-1573, June 2009.

[129] G. Zhang and X. Wang, "Girth-12 quasi-cyclic LDPC codes with consecutive lengths," CoRR, arxiv.org/abs/1001.3916.

[130] V. Zyablov and M. Pinsker, "Estimates of the error-correction complexity of Gallager's low-density codes," Problems of Inform. Transmission, vol. 11, no. 1, pp. 18-28, Jan. 1976.

[131] [Online] Available: http://cs.anu.edu.au/ bdm/nauty/

[132] [Online]. Available: http://www.inference.phy.cam.ac.uk/mackay/codes/ data.html

[133] [Online]. Available: http://csi.usc.edu/chugg/tools 


\section{Appendix A}

\section{Lemma 20 and its proof}

Lemma 20. The protograph code corresponding to the lifted graph constructed using the permutation shift matrix given in (5.6) applied to a fully-connected $3 \times n$ base graph with a lifting degree $N$ satisfying $N>(n-1) n$ has a minimum distance of at least 8 .

Proof. The proof consists of two parts. In Part I, we study the graphical structure(s) corresponding to a codeword of weight less than 8 . We show that for a Tanner graph with variable node degree of 3 and girth of 8 , it is impossible to have a codeword of weight less than 6 (Part I.A), or a codeword of weight 7 (Part I.B) in the corresponding code. In addition, in Part I.C, we prove that the only graphical structure corresponding to the nonzero variable nodes of a codeword of weight 6 is the one shown in Figure A.2. In Part II, we show that the structure of Figure A.2 cannot exist in the lifted graph constructed under the lemma's conditions. The combination of Parts I and II indicates that the minimum distance must be at least 8 .

Part I:

A. Codewords of weight less than or equal to 5: According to Lemma 22, for a regular Tanner graph with variable node degree 3 and girth 8 , the weight of a codeword cannot be less than 6 . This is obtained by substituting $g=8, d_{l}=3$ and $b=0$ ( $b$ is the number of unsatisfied check nodes) in Lemma 22.

B. Codewords of weight 7: By definition, every check node in the induced subgraph of a codeword, i.e., the subgraph induced by the nonzero variable nodes of a codeword, is connected to an even number of variable nodes. This implies that the total number 
of edges in the induced subgraph is an even number. In a regular graph with variable node degree of 3 (or in general, with an odd variable degree), any set containing an odd number of variable nodes is connected to an odd number of edges. Therefore, it is impossible to have a codeword of odd weight, e.g., 7, in such a code.

C. Codewords of weight 6: According to Lemma 22, for a regular Tanner graph with variable node degree 3 and girth 8 , all the check nodes in the induced subgraph of any codeword with weight less than 12 are connected to exactly 2 variable nodes. Now, consider the induced subgraph of a codeword of weight 6 in the code constructed under the conditions of the lemma, and suppose that this subgraph is grown from one of its variable nodes as the root. In the first layer, we have only one variable node (the root). In the second layer, we have 3 distinct check nodes. Similarly, the third and the fourth layers consist of 3 variable nodes and 6 check nodes, respectively (see Figure A.1). Note that according to Lemma 4 the girth of the graph is 8 , and thus

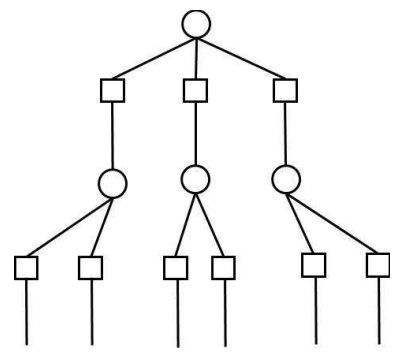

Figure A.1: The first four layers of the induced subgraph of a codeword of weight 6 , grown from one of its variable nodes.

all these variable/check nodes are distinct. Since we have 6 check nodes in Layer 4 , the number of variable nodes in the next layer is at least 2. As this is the subgraph of a codeword of weight 6 which already has 4 variable nodes in the previous layers, Layer 5 consists of exactly 2 variable nodes. Each of these variable nodes must be connected to check nodes with different parents in order to satisfy the girth condition (see Figure A.1). It is then easy to check that any subgraph satisfying these conditions has the structure of Figure A.2.

Part II:

In this part, we prove that it is impossible to have the structure of Figure A.2 in a graph under lemma's conditions. A careful inspection of Figure A.2 reveals 


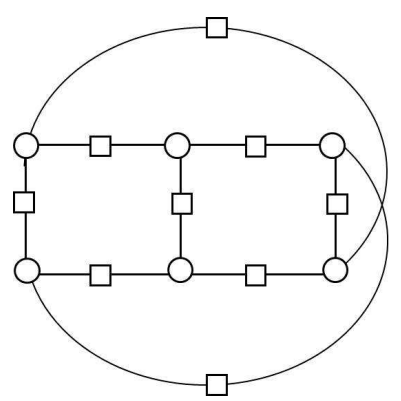

Figure A.2: The only possible structure for the induced subgraph of a codeword of weight 6 in a graph with variable degree 3 and girth 8 .

that there necessarily exists an 8-cycle in this graphical structure which contains two copies of the second check node (the check node corresponding to the second row of the permutation shift matrix) and one copy of each of the other two check nodes of the base graph. In the following, however, we show that under the lemma's conditions such an 8-cycle cannot exist in the lifted graph.

We recall that all the possible tailless backtrackless closed walks of length 8 in the base graph are covered in Figure 5.5. Since we are only interested in 8-cycles of the lifted graph with two copies of the second check node and one copy of each of the other two check nodes of the base graph, we just focus on Figures $5.5(b), 5.5(c)$ and $5.5(d)$. (Note that the structure in Figure 5.5(a) does not exist in a base graph with only 3 check nodes, and the structure in Figure 5.5(e) has two copies of only two of the check nodes of the base graph.) All the tailless backtrackless closed walks in Figures 5.5(b), 5.5(c) and 5.5(d) consist of two 4-cycles. The permutation shift of these walks can thus be expressed in terms of the permutation shifts of the constituent 4-cycles.

Consider the permutation shift of a 4 -cycle between the variable nodes $i_{1}$ and $i_{2}$ and the first two check nodes of the base graph. The permutation shift of this cycle is $0-0+i_{2}-i_{1}=i_{2}-i_{1} \bmod N$. The permutation shift of a 4 -cycle between variable nodes $i_{1}$ and $i_{2}$ and check nodes 1 and 3 is $0-0+\left(i_{2}-i_{1}\right) n=\left(i_{2}-i_{1}\right) n$ $\bmod N$. Similarly, the permutation shift of a 4 -cycle between variable nodes $i_{1}$ and $i_{2}$ and check nodes 2 and 3 is $\left(i_{2}-i_{1}\right)(n-1) \bmod N$. Now, consider Figures $5.5(b)$, $5.5(c)$ and $5.5(d)$ and let $w_{1}$ be the check node corresponding to the second row of the permutation shift matrix. The permutation shift of the corresponding tailless backtrackless closed walks can thus be expressed in the general form of $k_{1}(n-1) \pm k_{2}$ 
$\bmod N, 1 \leq k_{1}, k_{2} \leq n-1$. It is then easy to check that since $N>n(n-1)$, such permutation shifts cannot be zero. In other words, there will be no 8-cycle in the lifted graph whose image in the base graph consists of two copies of $w_{1}$, i.e., the second check node of the base graph, one copy of the first check node, and one copy of the third check node of the base graph. 


\section{Appendix B}

\section{Lemma 21 and its proof}

Lemma 21. Consider a $Q C$ protograph LDPC code constructed based on the following permutation shift matrix:

$$
P=\left[\begin{array}{ccccc}
0 & 0 & 0 & \ldots & 0 \\
\pi(0) & \pi(1) & \pi(2) & \ldots & \pi(n-1) \\
0 & n & 2 n & \ldots & (n-1) n
\end{array}\right]
$$

and a lifting degree $N$ satisfying $N>(2 n+1)(n-1)$. Suppose that $\pi$ in $(B .1)$ is a permutation over $\{0,1, \ldots, n-1\}$ with the property that $2 \pi\left(i_{2}\right) \neq \pi\left(i_{1}\right)+\pi\left(i_{3}\right)$ if $2 i_{2}=$ $i_{1}+i_{3}$ for distinct values of $i_{1}, i_{2}$, and $i_{3}{ }^{1}$ Then, the code has girth 8 and minimum distance of at least 10 .

Proof. The proof of having a girth 8 is very similar to that of Lemma 11. The only difference is that, here, the permutation shift of a 6 -cycle is either $d_{1}=\pi\left(i_{2}\right)$ $\pi\left(i_{3}\right)+n\left(i_{3}-i_{1}\right) \bmod N$ or $d_{2}=n\left(i_{2}-i_{3}\right)+\pi\left(i_{3}\right)-\pi\left(i_{1}\right) \bmod N$. Considering that $N>(2 n+1)(n-1)$ and that $i_{1}, i_{2}, i_{3}{ }^{2}$ as well as $\pi\left(i_{1}\right), \pi\left(i_{2}\right)$ and $\pi\left(i_{3}\right)$ are distinct integer numbers between 0 and $n-1$, it is easy to check that neither $d_{1}$ nor $d_{2}$ can be zero. The proof of not having a codeword of weight 6 in the lifted code also follows similar general steps as those of Lemma 20. Again, it is easy to check that, in the lifted graph constructed under lemma's constraints there cannot exist any 8-cycle

\footnotetext{
${ }^{1}$ An example of such a permutation for $n=8$ is $\pi(0,1,2,3,4,5,6,7)=(1,0,2,7,5,4,6,3)$.

${ }^{2}$ Note that here, we assume that the index of a variable node starts from 0 .
} 
whose image contains two copies of the second check node of the base graph and one copy of each of the other two check nodes. This is in contradiction with the graphical structure of the induced subgraph of a codeword with weight 6 . The proof of having no codeword of weight 8 has two parts. As the first part, it can be seen that the only structures corresponding to the induced subgraph of a codeword of weight 8 are those shown in Figure B.1. The proof for this claim follows similar steps as those described in the proof of Lemma 20 to conclude that the structure of Figure A.2 is the only possible structure for a codeword of weight 6 , and is thus omitted.

In the following, we show that, under the lemma's conditions, there cannot be any 8-cycle in the lifted graph which contains two copies of one of the check nodes of the base graph and one copy of each of the other two check nodes of the base graph. Moreover, there cannot be any 8-cycle which contains two copies of the second check node and two copies of the third check node of the base graph. These are in contradiction with the structures of Figure B.1.

Consider the permutation shifts of tailless backtrackless closed walks in Figures $5.5(b), 5.5(c)$ and $5.5(d)$. In the case that in these figures, $w_{1}$ represents the first check node of the base graph, the permutation shift of corresponding tailless backtrackless closed walks can be expressed in the general form of $k_{1} n \pm k_{2} \bmod N$, $1 \leq k_{1}, k_{2} \leq n-1$. In this case, if $N>(n+1)(n-1)$, the permutation shifts of such walks cannot be zero. That is there will be no 8-cycle in the lifted graph whose image in the base graph consists of two copies of the first check node of the base graph, one copy of the second and one copy of the third check node of the base graph. Similarly, in the case that $N>(2 n+1)(n-1)$, there will be no 8-cycle in the lifted graph whose image in the base graph consists of two copies of the third check node of the base graph, and one copy of each of the first and the second check nodes of the base graph. In the case that $w_{1}$ represents the second check node of the base graph, to avoid such tailless backtrackless closed walks with permutation shift zero, the lifting degree $N$ must satisfy $N>(n+2)(n-1)$. So for all three cases, the condition $N>(2 n+1)(n-1)$ of the lemma ensures that there will be no 8-cycle in the lifted graph with the corresponding properties.

Here, we also show that, it is impossible to have an 8-cycle in the lifted graph whose image contains two copies of the second check node and two copies of the third 


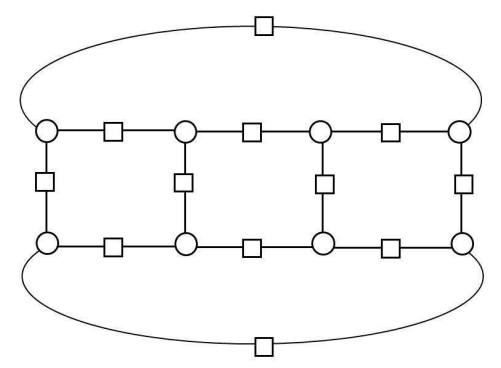

$(a)$

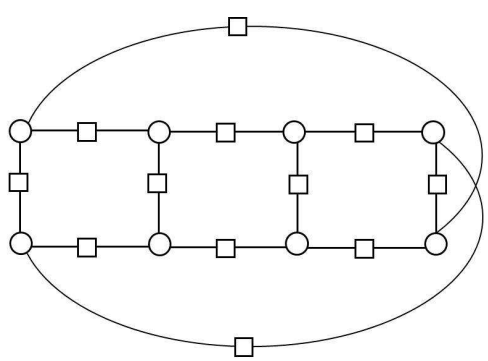

(b)

Figure B.1: The only possible structures for the induced subgraph of codewords with weight 8 in a regular Tanner graph with variable node degree 3 and girth 8 .

check node of the base graph. Among all the possible structures discussed in Figure 5.5 for tailless backtrackless closed walks of length 8 , the only possible structures which can contain only two check nodes of the base graph are (a) a modification of Figure 5.5(d) in which variable nodes and check nodes are switched, and (b) the structure of Figure 5.5(e). Case (a) is shown in Figure B.2. In the case that in Figure B.2, $w_{2}$ and $w_{3}$ are the check nodes corresponding to the second and the third rows of the permutation shift matrix, the permutation shift of the tailless backtrackless closed walk $w_{2}, u_{i_{2}}, w_{3}, u_{i_{1}}, w_{2}, u_{i_{2}}, w_{3}, u_{i_{3}}, w_{2}$ is:

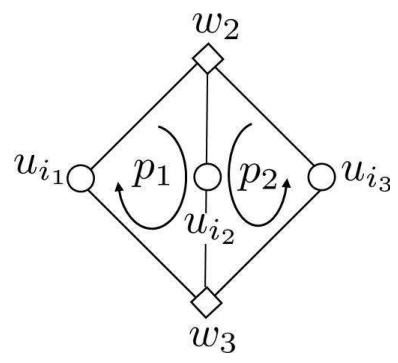

Figure B.2: Graphical structure of tailless backtrackless closed walks of length 8 containing 2 copies of each of the check nodes $w_{2}$ and $w_{3}$ of the base graph.

$$
\begin{aligned}
p=p_{1}+p_{2} & =\left(\pi\left(i_{2}\right)-i_{2} n+i_{1} n-\pi\left(i_{1}\right)\right)+\left(\pi\left(i_{2}\right)-i_{2} n+i_{3} n-\pi\left(i_{3}\right)\right) \\
& =\left(i_{1}+i_{3}-2 i_{2}\right) n+\left(2 \pi\left(i_{2}\right)-\pi\left(i_{1}\right)-\pi\left(i_{3}\right)\right) .
\end{aligned}
$$


Considering the lemma's conditions, $p$ is non-zero. Similarly, it can be shown that, under the conditions of the lemma, the permutation shifts of all the other possible tailless backtrackless closed walks of length 8 in Figure B.2 are non-zero. It is also easy to check that for the Case (b) where the two check nodes correspond to the second and the third rows of the permutation shift matrix, under the condition of the lemma, all the tailless backtrackless closed walks of length 8 have non-zero permutation shifts. Therefore, it is impossible to have an 8-cycle in the lifted graph whose image contains two copies of the second check node and two copies of the third check node of the base graph.

The proof for the contradiction of the above results with the structures in Figure B.1 follows similar steps as those described in the second part of the proof of Lemma 20, and is thus omitted. 


\section{Appendix C}

\section{Lemma 22 and its proof}

Lemma 22. For a left-regular graph $G$ with left degree $d_{l} \geq 3$ and girth $g>4,{ }^{1}$ consider an $(a, b)$ trapping set with $b<a$. If such a trapping set is elementary, let the notation $a_{e}$ denote its size, and consider the case where $d_{l}\left(d_{l}-1\right)>b$. Otherwise, for non-elementary trapping sets with $b<a$, let the notations $a_{n 1}$ and $a_{n 2}$ denote the size of the trapping set if it has at least one unsatisfied check node of degree $d_{o}>1$ and one satisfied check node of degree $d_{e}>2$ in $G(\mathcal{S})$, respectively. For the two latter cases, suppose that $d_{o}\left(d_{l}-1\right)>b$ and $d_{e}\left(d_{l}-1\right)>b$, respectively. Then depending on the value of $g$, we have the following two sets of inequalities:

a) For $g=4 k$, where $k$ is an integer larger than 1, we have:

$$
\begin{gathered}
a_{e} \geq 1+d_{l}+\left(d_{l}\left(d_{l}-1\right)-b\right) \sum_{i=0}^{k-3}\left(d_{l}-1\right)^{i}+ \\
\frac{\left(d_{l}\left(d_{l}-1\right)-b\right)\left(d_{l}-1\right)^{k-2}}{d_{l}} \\
a_{n 1} \geq d_{e}+\left(d_{e}\left(d_{l}-1\right)-b\right) \sum_{i=0}^{k-2}\left(d_{l}-1\right)^{i}
\end{gathered}
$$

\footnotetext{
${ }^{1}$ For the case of $d_{l}=2$, it is easy to see that any $(a, b)$ elementary trapping set has $b=0$ or $b=2$. For $b=0$, the smallest value of $a$ is $g / 2$, which corresponds to the trapping set being a shortest cycle. For an elementary trapping set with $b=2$, the smallest value of $a$ is one, which corresponds to a single variable node. For a non-elementary $(a, b)$ trapping set however, if $b=0$, the smallest value of $a$ is $g$. If $b=2$, the minimum value of $a$ for such a trapping set is 3 .
} 


$$
a_{n 2} \geq d_{o}+\left(d_{o}\left(d_{l}-1\right)-b+1\right) \sum_{i=0}^{k-2}\left(d_{l}-1\right)^{i}
$$

b) For $g=4 k+2$, where $k$ is a positive integer, we have:

$$
\begin{gathered}
a_{e} \geq 1+d_{l}+\left(d_{l}\left(d_{l}-1\right)-b\right) \sum_{i=0}^{k-2}\left(d_{l}-1\right)^{i} \\
a_{n 1} \geq d_{e}+\left(d_{e}\left(d_{l}-1\right)-b\right) \sum_{i=0}^{k-2}\left(d_{l}-1\right)^{i}+ \\
\frac{\left(d_{e}\left(d_{l}-1\right)-b\right)\left(d_{l}-1\right)^{k-1}}{d_{l}} ; \\
a_{n 2} \geq d_{o}+\left(d_{o}\left(d_{l}-1\right)-b+1\right) \sum_{i=0}^{k-2}\left(d_{l}-1\right)^{i}+ \\
\frac{\left(d_{o}\left(d_{l}-1\right)-b+1\right)\left(d_{l}-1\right)^{k-1}}{d_{l}}
\end{gathered}
$$

Proof. Here, we just present the sketch of the proof. For this, we first need the following lemma, whose proof is given in Appendix D.

Lemma 18(i): In a left-regular graph $G$ with left degree $d_{l} \geq 2$, if the induced subgraph $G(\mathcal{S})$ of an $(a, b)$ trapping set $\mathcal{S}$ does not contain any cycle, then $b \geq$ $a\left(d_{l}-2\right)+2$. The inequality is satisfied with equality for elementary trapping sets.

Based on Lemma 18(i), it is clear that a trapping set with $b<a$ has at least one cycle. Therefore, considering any variable (or check) node of $\mathcal{S}$ as the root, and growing $G(\mathcal{S})$ from that node, one can construct a tree of at least $g / 2$ layers, where the layers contain either variable or check nodes alternately, with no repetition of nodes. The number of variable nodes in this tree can be used as a lower bound on the number of variable nodes in $\mathcal{S}$. In this tree, the number of check nodes in layer $i>1$ of the tree, $N_{c}^{i}$, is $N_{c}^{i}=\left(d_{l}-1\right) N_{v}^{i-1}$, where $N_{v}^{i-1}$ is the number of variable nodes in layer $i-1$. Similarly, $N_{v}^{i}=\sum\left(d_{c_{j}^{i-1}}-1\right)$, where $d_{c_{j}^{i-1}}$ is the degree (within $G(\mathcal{S})$ ) of the $j$ th check node in layer $i-1$, and the summation is over all the check nodes in layer $i-1$. To minimize the number of variable nodes in the tree, one needs to make $\sum\left(d_{c_{j}^{i-1}}-1\right)$ as small as possible in each check node layer of the tree. In 
particular, this should be done at the first (upper) layer(s) of the tree if possible, since these layers contribute the most in the total number of variable nodes in the tree. In addition, to obtain a lower bound on the size of the trapping sets, we assume that even for the non-elementary case, except for one check node, the degrees of all the other check nodes in $G(\mathcal{S})$ are either 1 or 2 . Moreover, we assume that all the check nodes of degree 1 are in the first (upper) layer(s) of check nodes after the root layer.

For the case of an elementary trapping set, according to the assumption of $b<a$, there is at least one variable node that is not connected to any unsatisfied check nodes. Considering such a variable node as the root node, all the check nodes in the first layer are satisfied check nodes. That is, $N_{v}^{0}=1$ (root node), $N_{c}^{1}=N_{v}^{2}=d_{l}$, $N_{c}^{3}=d_{l}\left(d_{l}-1\right), N_{v}^{4}=d_{l}\left(d_{l}-1\right)-b$ and $N_{c}^{i-1}=\left(d_{l}-1\right) N_{v}^{i-2}, N_{v}^{i}=N_{c}^{i-1}$, for $i=6,8, \ldots{ }^{2}$ Therefore, the total number of variable nodes in the constructed tree is $1+d_{l}+\left(d_{l}\left(d_{l}-1\right)-b\right)+\left(d_{l}\left(d_{l}-1\right)-b\right)\left(d_{l}-1\right)+\ldots$ Distinction should be made between the cases of $g=4 k+2$ and $g=4 k$. While in the former, the last layer of the tree consists of variable nodes, in the latter, it consists of check nodes. In the case where the last layer consists of check nodes, for each set of $d_{l}$ check nodes in the last layer of the tree, there must be at least one other variable node in $\mathcal{S}$. The sketch of the proofs for the non-elementary cases are similar to that of the elementary case, with the difference that the check node of degree $d_{o}$ or $d_{e}$ is used as the root node.

\footnotetext{
${ }^{2}$ Here, based on the statement of the lemma, we have assumed that all the unsatisfied check nodes can fit in the third layer of the tree. In the case that $d_{l}\left(d_{l}-1\right)-b \leq 0$, some of the unsatisfied check nodes have to be located in the next layer(s), and the above equations and the claims of the lemma will have to be accordingly revised.
} 


\section{Appendix D}

\section{Proof of Lemma 18(i)}

Proof of Lemma 18(i):

Since $G(\mathcal{S})$ does not contain any cycle, it forms a tree (note that $G(\mathcal{S})$ is connected). Suppose that $G(\mathcal{S})$ is grown from a variable node of $\mathcal{S}$ as the root, one layer at a time, until along each path, the growth is terminated by reaching a check node as a leaf. These nodes are the unsatisfied check nodes of degree one. In the tree, each variable node, except the root, has a parent which is a check node of degree at least 2 . In the case that $\mathcal{S}$ is elementary, the degree of the parent check nodes is 2 , and hence each check node is the parent to one variable node. There are thus exactly $a-1$ check nodes of degree 2 in $G(\mathcal{S})$. Since $G(\mathcal{S})$ is a tree, the number of its nodes equals the number of its edges plus one. The total number of nodes in the graph is $a+(a-1)+b_{1}$ and the total number of edges is $a \cdot d_{v}$, where $b_{1}$ is the number of unsatisfied check nodes of degree one. For an elementary trapping set, we thus have $2 a+b_{1}-1=a d_{v}+1$, which implies $b=b_{1}=a\left(d_{v}-2\right)+2$. In the case that $\mathcal{S}$ is not elementary, some variable nodes may share the same parent. The number of parent check nodes is thus less than $a-1$, and therefore $b \geq b_{1}>a\left(d_{v}-2\right)+2$. 


\section{Appendix E}

\section{Lemma 23 and its proof}

Lemma 23. Let $G=(L \cup R, E)$ be a left-regular bipartite graph with left degree 2 . Consider a set $\mathcal{S} \in L$, for which the induced subgraph is a tree and has the longest path of length $2 k-2$. Then $|\mathcal{S}| \leq \sum_{i=0}^{k-2}\left(d_{c, \text { max }}-1\right)^{\left\lfloor\frac{i+1}{2}\right\rfloor}$, where $|\mathcal{S}|$ is the number of nodes in $\mathcal{S}$ and $d_{c, \text { max }}$ is the maximum degree of the nodes in $R$.

Proof. The upper bound is derived by counting the number of variable nodes in a tree where the number of check nodes is maximized with the constraint that the longest path has $2 k-2$ edges. This implies that there is a path of length $2 k-2$ between any two leaf check nodes of $G(\mathcal{S})$. In addition, to maximize $|\mathcal{S}|$, the degree of all the check nodes in $G(\mathcal{S})$ is assumed to be $d_{c, \max }$. 